LPSC-08-08

LYCEN-2008-1

To appear in "Physics Reports"

\title{
SPIN OBSERVABLES AND SPIN STRUCTURE FUNCTIONS: INEQUALITIES AND DYNAMICS
}

\author{
Xavier Artru \\ Université de Lyon, IPNL \\ Université Lyon 1 and CNRS/IN2P3 \\ 69622 Villeurbanne, France \\ email: xavier.artrueipnl.in2p3.fr \\ Mokhtar Elchikh \\ Université des Sciences et de Technologie d'Oran \\ Département de Physique, BP 1505 \\ El Menauoer, Oran, Algeria \\ email: elchikh@yahoo.com \\ Jean-Marc Richard \\ Laboratoire de Physique Subatomique et Cosmologie \\ Université Joseph Fourier, CNRS/IN2P3, INPG \\ 53, avenue des Martyrs, 38026 Grenoble, France \\ email:jean-marc.richardelpsc.in2p3.fr \\ Jacques Soffer \\ Physics Department, Temple University \\ Barton Hall, 1900 N. 13th Street \\ Philadelphia, PA 19122 -6082, USA \\ email: jsofferatemple.edu

\section{Oleg V. Teryaev} \\ Bogoliubov Laboratory of Theoretical Physics, \\ Joint Institute for Nuclear Research, \\ 141980 Dubna, Moscou region, Russia \\ email: teryaevetheor.jinr.ru
}

June 7, 2018 


\begin{abstract}
Model-independent identities and inequalities which relate the various spin observables of collisions in nuclear and particle physics are reviewed in a unified formalism. Their physical interpretation and their implications for dynamical models are also discussed. These constraints between observables can be obtained in several ways: from the explicit expression of the observables in terms of a set of helicity or transversity amplitudes, a non-trivial algebraic exercise which can be preceded by numerical simulation with randomly chosen amplitudes, from anticommutation relations, or from the requirement that any polarisation vector is less than unity. The most powerful tool is the positivity of the density matrices describing the spins in the initial or final state of the reaction or its crossed channels. The inequalities resulting from positivity need to be projected to single out correlations between two or three observables. The quantum aspects of the information carried by spins, in particular entanglement, are considered when deriving and discussing the constraints

Several examples are given, with a comparison with experimental data in some cases. For the exclusive reactions, the cases of the strangeness-exchange proton-antiproton scattering and the photoproduction of pseudoscalar mesons are treated in some detail: all triples of observables are constrained, and new results are presented for the allowed domains. The positivity constraints for total cross-sections and for the simplest observables of single-particle inclusive reactions are reviewed. They also apply to spin-dependent structure functions and parton distributions, both integrated or transverse-momentum dependent. The corresponding inequalities are shown to be preserved by the evolution equations of quantum chromodynamics.
\end{abstract}




\section{Contents}

1 Introduction $\quad 5$

2 Basic formalism $r$

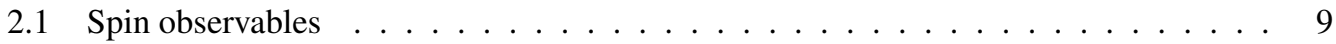

2.1.1 Kinematics and frames . . . . . . . . . . . . . . . . 9

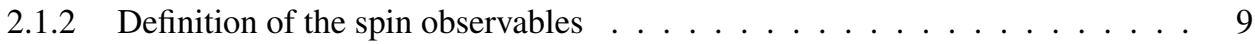

2.1.3 Centre-of-mass and other frames . . . . . . . . . . . . . . 11

2.2 General properties of density matrices . . . . . . . . . . . . . . . 11

2.2 .1 Definitions ...................... 11

2.2.2 The positivity conditions . . . . . . . . . . . . . . . 12

2.2.3 The full domain of positivity . . . . . . . . . . . . . . . 13

2.2.4 Structure of the boundary of $\mathcal{D} \ldots \ldots \ldots \ldots$

2.2.5 The projected positivity domains . . . . . . . . . . . . . . 17

2.3 Spin states of a single particle . . . . . . . . . . . . . . . . 18

2.3.1 Spin $1 / 2 \ldots \ldots \ldots \ldots \ldots$

2.3.2 Generalities on photon polarisation . . . . . . . . . . . . 18

2.3.3 Massive vector particle . . . . . . . . . . . . . . . . . 19

2.3.4 Phase conventions for the helicity and transversity states . . . . . . . . . 22

2.4 Joint density matrix of several particles . . . . . . . . . . . . . . . 22

2.5 Description of the reactions . . . . . . . . . . . . . . . . . . . . . . . . . . . . . .

2.5.1 The Cross Section Matrix . . . . . . . . . . . . . . . 24

2.5.2 Reduced cross section matrices ................. 25

2.5.3 Physical meaning of the positivity conditions . . . . . . . . . . . 26

2.6 Search for the positivity domain of a subset of observables . . . . . . . . . . . 27

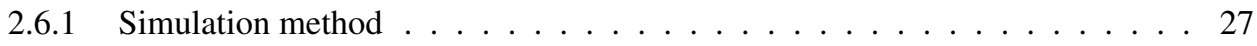

2.6.2 Anticommutation and spherical constraints. . . . . . . . . . . . . 27

2.6.3 Number of amplitudes and existence of constraints . . . . . . . . . . 28

2.6.4 Methods of the apparent contour and of the reciprocal polar transform . . . . 28

2.7 Quadratic identities between observables . . . . . . . . . . . . . . . . . . . 29

2.8 Symmetry constraints on observables . . . . . . . . . . . . . . . . . . . . 29

2.8.1 Rotation invariance (conservation of angular momentum) . . . . . . . . . . 29

2.8.2 Parity conservation (in strong and electromagnetic interactions) . . . . . . 30

2.8.3 Permutations of $A \leftrightarrow B$ and/or $C \leftrightarrow D \ldots \ldots . \ldots . \ldots . \ldots 32$

2.8 .4 Identical particles . . . . . . . . . . . . . . . . . 33

2.8.5 Charge and $C P$ conjugations in $A+\bar{A} \rightarrow B+\bar{B} \ldots \ldots . . . . . . .34$

2.8.6 Time reversal invariance in $A+B \rightarrow A+B \ldots \ldots 35$

2.8.7 Hermitian scattering matrix ................. 36

2.8 .8 Crossed reactions . . . . . . . . . . . . . . . . . . 37

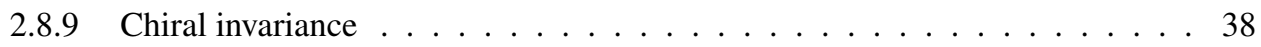

2.8 .10 Inequalities relating isospin-partner reactions $\ldots \ldots . \ldots$

3 Exclusive reactions $\quad 41$

3.1 Pion-nucleon elastic scattering . . . . . . . . . . . . . . . . . 41

3.2 Antiproton-proton annihilation into two pseudoscalar mesons . . . . . . . . . 43

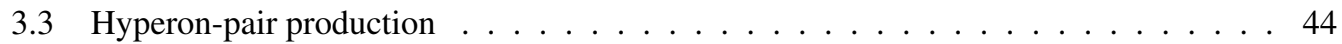

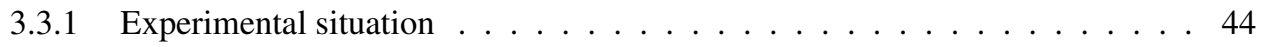

3.3.2 Formalism of hyperon-pair production . . . . . . . . . . . . . 46

3.3 .3 Empirical approach . . . . . . . . . . . . . . . . . 48

3.3.4 Understanding the observed domains . . . . . . . . . . . . . . 56

3.3.5 Special cases ...................... 61 
3.3.6 Proton-proton scattering and other similar reactions . . . . . . . . . . . 61

3.4 Photoproduction of scalar or pseudoscalar mesons . . . . . . . . . . . . . . 63

3.4 .1 Experimental results . . . . . . . . . . . . . . . . 63

3.4 .2 Amplitudes . . . . . . . . . . . . . . . . . . . . 64

3.4 .3 The observables . . . . . . . . . . . . . . . . . . . 65

3.4.4 The Cross Section Matrix . . . . . . . . . . . . . . . . . 66

3.4 .5 Inequalities . . . . . . . . . . . . . . . . . 67

3.4 .6 Non-linear identities . . . . . . . . . . . . . . . . . . . . . . . . 68

3.4 .7 Symmetry rules . . . . . . . . . . . . . . . . . . . . 69

3.4 .8 Future applications $\ldots \ldots \ldots \ldots \ldots$

3.5 Photoproduction of vector mesons $\ldots \ldots \ldots \ldots \ldots$

3.5 .1 Experimental situation . . . . . . . . . . . . . . . 70

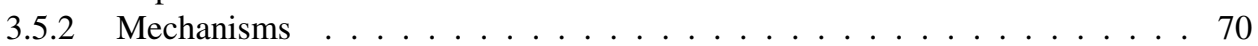

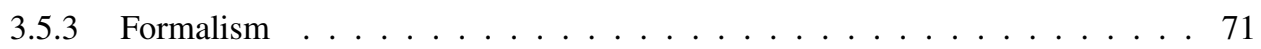

3.5.4 Inequalities for the case of unpolarised baryons . . . . . . . . . . . . . 73

3.5.5 Inequalities for polarised baryons . . . . . . . . . . . . . . . 75

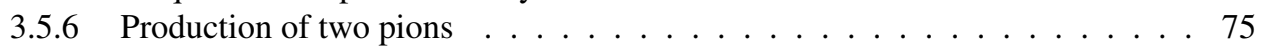

4 Inclusive reactions $\quad \mathbf{7 7}$

4.1 Spin observables in hadronic inclusive reactions . . . . . . . . . . . . . . 77

4.1 .1 Total cross sections . . . . . . . . . . . . . . . . . . . . . . 77

4.1 .2 Spin transfer observables . . . . . . . . . . . . . . . . . . 78

4.1 .3 Initial spin observables . . . . . . . . . . . . . . . . . 80

4.2 Spin observables in deep inelastic scattering and structure functions . . . . . . . . 81

4.2.1 Positivity constraint for the $A_{2}$ transverse asymmetry . . . . . . . . . 81

4.2.2 Positivity Constraints for Structure Functions . . . . . . . . . . . . . . 83

4.2.3 Neutrino Deep Inelastic Scattering . . . . . . . . . . . . . . . . . . . . 85

4.2 .4 Photon structure functions . . . . . . . . . . . . . . . . . 86

4.3 Positivity constraints for parton distributions and fragmentation functions . . . . . 91

4.3.1 Parton distributions and fragmentation functions (integrated over transverse momentum) . . . . . . . . . . . . . . . . . . 92

4.3.2 Spin and transverse-momentum dependent quark distributions . . . . . . . 9 95

4.3.3 Generalised Parton Distributions . . . . . . . . . . . . . . . . . . . . . 97

4.3.4 QCD Evolution of Positivity Constraints . . . . . . . . . . . . . . . . . 98

5 Further developments $\quad 107$

5.1 Polarised cascades . . . . . . . . . . . . . . . . . . . . . . 107

5.1 .1 Dual types of density matrices . . . . . . . . . . . . . . . . . 107

5.1.2 Monte-Carlo simulations of entangled cascades . . . . . . . . . . . . 107

5.2 Quantum information aspects . . . . . . . . . . . . . . . . . . 108

5.2 .1 Various kinds of entropy . . . . . . . . . . . . . . . . . . . 109

5.2.2 Entropy of the cross section matrix . . . . . . . . . . . . . . 110

5.2.3 Factorisation, separability or entanglement of the cross section matrix . . . 111

5.2.4 Entropy of the observed final state . . . . . . . . . . . . . . . . 111

5.2.5 Transfer of information from initial to final state . . . . . . . . . . . 112

5.3 Domains of quantum positivity, classical positivity and separability . . . . . . . 113 


\section{Introduction}

The ultimate tests of the dynamics of the standard model and the low-energy hadron interaction require the probing of the spin degrees of freedom. The developments on beam, target and detector technology make it now possible to perform scattering experiments with polarised beam and target, and to measure the polarisation of the outgoing particles. For instance, the elastic nucleon-nucleon scattering has been studied with beam and target both polarised. At CERN, the strangeness-exchange reaction $\overline{\mathrm{p} p} \rightarrow \bar{\Lambda} \Lambda$ has been measured with a polarised hydrogen target, and thanks to the weak decay of the hyperon, the polarisation of both $\bar{\Lambda}$ and $\Lambda$ has been measured.

These developments are also applied to inclusive reactions $a+b \rightarrow c+X$, with $a, b$ and $c$ carrying spin, and measurements of spin observables up to rank three can be envisaged, if the beam $a$ and target $b$ are polarised and the polarisation of the produced particle $c$ is analysed.

Significant progress has been achieved recently in the investigation of spin observables, spindependent structure functions and parton distributions, in particular at RHIC, Jlab, GRAAL, CERN and DESY. New data are expected from presently running experiments and further measurements are expected at the future facilities such as the hadron factories at KEK (Japan) and GSI (Germany).

This experimental progress has stimulated an important theoretical activity. In the eighties, the so-called "spin crisis" (one should say "helicity crisis") has motivated the high-energy theorists to study the spin aspects of parton distributions and a large effort is now devoted to the quark transverse spin ("transversity") and the T-odd parton distributions.

Spin physics will probably benefit from significant progress in the future. As an example, polarised electrons and positron beams are planned for the International Linear Collider (ILC) to reduce the backgrounds and to improve the sensitivity to new physics. Attempts are made to design polarisation devices for antiprotons which, if successful, will enlarge the physics program of the GSI hadron facility.

The physics case The merit of spin observables for a detailed understanding of the underlying dynamics has been often demonstrated and hardly needs to be stressed once more in great detail (see, for instance, Refs. [1,2]). Let us give one example: in the conventional theory of nuclear forces, pion-exchange has a spin-spin and a tensor component, this latter acting with specific strength on each of the spin-triplet $\mathrm{P}$-wave states of scattering, ${ }^{3} \mathrm{P}_{0},{ }^{3} \mathrm{P}_{1},{ }^{3} \mathrm{P}_{2}$. The pattern induced by pionexchange is present at very low energy, but, as the incoming energy increases, a departure is clearly observed. This led Breit and others to introduce a spin-orbit component in the interaction, and to speculate about the existence of vector mesons whose exchange is responsible for this important spin-orbit term in the potential. These $\rho$ and $\omega$ mesons were, indeed, discovered. See for instance, Refs. [3,4].

In scattering experiments, the integrated cross-section and the main trend of the angular distribution are dominated by simple basic mechanisms, for instance attraction or absorption. To reach more subtle contributions that provide signatures for the more elaborated models, one needs to apply filters. This is the role of spin observables.

For instance, in the strangeness-exchange reaction $\bar{p} p \rightarrow \bar{\Lambda} \Lambda$, a picture based on the exchange of mesons (K, $\mathrm{K}^{*}$, etc.) and a model based on $\bar{u} u$ annihilation and $\bar{s} s$ creation were both able to describe the first data taken at LEAR on angular distribution, polarisation and spin-correlation coefficients of final-state hyperons. However, these models gave different predictions for the depolarisation and spin transfer coefficients that became available once a polarised hydrogen target was installed.

Experimental situation Some reactions have received special attention on the experimental side. In the low-energy domain, this is the case for nucleon-nucleon scattering. A comprehensive formalism has been written down [5]. Delicate experiments, in particular at Saclay (Saturne) and Villigen (PSI), have enabled one to tune the potential models, later on used in nuclear-structure calculations and phase-shift analyses, and eventually a direct reconstruction of the amplitudes. A summary of 
most of this work can be found in a review by Leluc and Lehar $[6]^{1}$. More recent measurements have been performed, e.g., by the PINTEX collaboration at the Indiana Cyclotron [7-9]; by the EDDA collaboration at COSY [10-12]; at JINR, Dubna [13]; etc.

Thanks to this detailed knowledge of the scattering observables, models have been constructed for the nucleon-nucleon potential and used to study more complicated systems from tritium to neutron stars. A milestone was the Paris potential [14]. An example of more recent and more sophisticated development is the so-called "CD Bonn" model [15]. Chiral models have also been developed which hopefully will lead to a unified picture of long- and short-range contributions, see, e.g., [16].

Basic mechanisms of the low-energy hadronic interaction are tested in scattering experiments involving polarised light nuclei or polarised photon beams. Spin physics is very much active in particular at Jlab [17-19]: the CLAS collaboration has just measured the polarisation, and beam-torecoil spin transfer in the photoproduction process $\gamma \mathrm{N} \rightarrow \mathrm{K} \Lambda$, a reaction also studied by the GRAAL collaboration at Grenoble. Interestingly, the data saturated the inequalities (which will be reviewed in Section 3), indicating that some of the transversity amplitudes are dominant, which sheds some light on the underlying mechanism. Kloet et al. [20-22] have analysed some inequalities on the observables relevant for the photoproduction of vector mesons, and their subsequent decay into two pseudoscalar mesons or a pair of leptons.

The physics of low-energy antiprotons has been particularly active in the 80's and 90's at the LEAR facility of CERN. The analysing power $A_{y}$ of the annihilation reactions into two pseudoscalars, $\overline{\mathrm{p}}+\mathrm{p} \rightarrow \pi^{-} \pi^{+}$and $\overline{\mathrm{p}}+\mathrm{p} \rightarrow \mathrm{K}^{-} \mathrm{K}^{+}$has revealed a wide kinematical region with nearly maximal values $\left|A_{y}\right| \sim 1$. This will be discussed later in this review.

The reactions $\overline{\mathrm{p}}+\mathrm{p} \rightarrow \overline{\mathrm{Y}}+\mathrm{Y}^{\prime}$, where $\mathrm{Y}$ denotes a hyperon $(\Lambda, \Sigma, \Xi)$ have been much studied at LEAR. Information on the spin of the final-state particles is provided by their decay. Here several spin observables can be accessed, and some rank-three observables were obtained in the last runs for which a polarised target was installed. The formalism for the reaction $\bar{p} p \rightarrow \bar{\Lambda} \Lambda$ will be discussed at length in this review. In 1964, i.e., much before the LEAR experiments, some subtleties of the spin observables were analysed [23,24]. In particular, Cohen-Tannoudji and Messiah noted that (for $\mathrm{Y}=\mathrm{Y}^{\prime}$ ) accurate data can indicate whether or not the antiproton beam is polarised [24].

Several models were proposed for these strangeness-exchange reactions, with a renewed activity when the threshold behaviour was analysed at LEAR, first with an unpolarised target, by the PS185 collaboration. These early LEAR data indicated a sizeable amount of polarisation and spin correlation in the final state, in particular, a vanishing spin-singlet fraction for $\bar{p} p \rightarrow \bar{\Lambda} \Lambda$. Unfortunately these first data did not enable to distinguish between the quark-inspired models and the kaon-exchange models à la Yukawa, which all gave a fairly good agreement. It was then suggested that a polarised target might help distinguishing among the possible mechanisms. Holinde et al., and others (see, e.g., [25] for references), claimed that quark models and kaon-exchange models give different predictions for some observables, such as $D_{n n}$, that become accessible with a polarised target. Predictions on these observables were also made by Alberg et al. with in particular a very large transversity-flip of the baryon, i.e., a large negative depolarisation parameter $D_{n n} \simeq-1$, if the strange quark pair is extracted from the sea of the proton or antiproton [26]. It was then realised [27] that the existing data already constrain the depolarisation to a small interval near $D_{n n}=0$. The data taken with a transversely polarised target, gave, indeed, values of $D_{n n}$ close to 0 , as it shown in the next section. A more systematic study of the correlation among the observables in this reaction $\overline{\mathrm{p}} \mathrm{p} \rightarrow \bar{\Lambda} \Lambda$ was made in [28-31].

Spin physics at very high energy is now investigated thanks to the polarised proton-proton collider in operation at RHIC-BNL since 2002 [32]. It will allow to test the spin sector of perturbative QCD and to measure the parton distributions, in particular, the transversity distribution whose relevance was recognised in the early 90 's. This is opening a new area which will bring a lot of insight in the nucleon spin structure, a fundamental problem which came up only 20 years ago.

Large single spin asymmetries in hadronic reactions at high energies have been already observed

\footnotetext{
${ }^{1}$ We thank Catherine Leluc for a very informative discussion on the subject
} 
and they are among the most challenging phenomena to understand from basic principles in QCD. In particular it was shown recently that initial or final interactions, due to the exchange of a gluon, generate the theoretical mechanism to explain transverse spin asymmetries [33] and outstanding spin anomalies like the observed violation of the Lam-Tung relation in the angular distribution of the leptons in the unpolarised $\pi-\mathrm{N}$ Drell-Yan process $[34,35]$.

Identities and inequalities The analysis of experimental data and their interpretation is greatly helped by model-independent constraints (identities and inequalities) between the various observables. These constraints also help in planning the future experimental programs by pointing out the redundant measurements.

When all possible spin observables are considered, linear and quadratic identities can be written. Linear identities reflect the symmetries such as parity, time reversal, identical particles, etc. For instance, in elastic scattering, the analysing power (azimuthal asymmetry of the angular distribution with reference to the target polarisation) coincides with the spontaneous polarisation of the recoiling particle. Such identities can also be written when the spins of some of the particles are not measured. Quadratic identities relating observables are known for years, such as $P_{n}^{2}+A^{2}+R^{2}=1$ for the polarisation and spin-rotation parameters of pion-nucleon scattering, with a notation to be defined more precisely later. Deriving these non-linear equalities can be seen as a purely algebraic (but non trivial) exercise, once the various spin observables are expressed in terms of a set of amplitudes.

Spin observables are also constrained by inequalities, which express the positivity of some polarised cross sections, sometimes in a gedanken experiment involving entangled spin states as we shall show in Section 2. For one observable we have "trivial" bounds, for instance $\left|A_{n}\right| \leq 1$ for the analysing power, $\left|P_{n}\right| \leq 1$ for the spontaneous final polarisation and $\left|D_{n n}\right| \leq 1$ for the socalled depolarisation coefficient. For a pair $\left\{C_{1}, C_{2}\right\}$ of observables, each of which satisfy $\left|C_{i}\right| \leq 1$, positivity can restrict the allowed domain to a subset of the square $[-1,+1]^{2}$. Similarly a triple $\left\{C_{1}, C_{2}, C_{3}\right\}$ of observables is often constrained to a subset of the cube $[-1,+1]^{3}$, etc. Such inequalities provide model-independent constraints on observables which are measured independently. They also indicate which of the yet-unknown observables offer the widest range of variation for the further checking of the models. If, for instance, an inequality $C_{1}^{2}+C_{2}^{2} \leq 1$ has been derived, and $C_{1}$ safely measured to be close to -1 in a certain domain of kinematics, it is no longer necessary to device a new experiment for measuring $C_{2}$. The aim of this review is to show how to derive and understand these constraints, expressed in terms of simple inequalities.

The inequalities discussed in this review precisely indicate whether new observables are necessary or are already much constrained by the existing ones. We shall give other examples in Sec. 4 , dealing with the inclusive reactions. In this category we put also the positivity bound for transversity parton distributions, which will be derived in Sec. 4.3.1.

A brief history of spin identities and inequalities. The idea that spin observables are constrained by positivity conditions is far from being new. Some milestones are now presented of the contributions of which we are aware. We apologise for the important contributions which we may have inadvertently omitted.

In 1958, Lee and Yang [36] studied the decay symmetry of the $\Lambda^{0}$ and established constraints in the light of which the experimental data suggested that $\Lambda^{0}$ has spin $1 / 2$.

Positivity conditions have been written down for the density matrix describing spin-one meson resonances and spin $3 / 2$ baryon resonances [37].

In 1965, Ademollo, Gatto and Preparata proposed more general tests based on the properties of the density matrix for a two-body inelastic collision, to determine the spins of the final state particles [38].

In the 60's, basic contributions were elaborated by Louis Michel and his collaborators. See, in particular [39-42] about the "polarisation domain". Other references of interest are [24,43].

In 1975, Delanay and Gammel [44] and, independently, Bourrely and Soffer [45] wrote down the quadratic relations among the spin observables of the proton-proton elastic scattering. Reading 
the book by Leader on spin physics [2] tells us that similar results were obtained earlier by Klepikov, Kogan and Shamanin [46]. The art of identities among observables has been further developed, in particular by La France and Winternitz [47].

Various derivations of the inequalities. For the exclusive reactions, many positivity conditions are just a consequence of the identities mentioned above. For instance $P_{n}^{2}+A^{2}+R^{2}=1$ implies $A^{2}+R^{2} \leq 1$ and $\left|P_{n}\right| \leq 1$. Some inequalities can be derived algebraically starting from the the explicit expression of observables $C_{1}, C_{2}, \ldots$ in terms of amplitudes $a, b, \ldots$; however this exercise that looks easy at first sight, turns out rather involved. The search for the inequalities can be guided by generating randomly artificial amplitudes and plotting one observable against another one. This gives a first image of the allowed domain for the observables. Once an inequality is guessed from the plot, one may try to derive it rigorously.

Spherical inequalities of the type $C_{1}^{2}+C_{2}^{2}+\ldots C_{n}^{2} \leq 1$ can be quickly obtained from the anticommutation properties of the observables (see Sec. 2). More systematically, the inequalities result from the positivity of the density matrix of the set or a subset of initial or final particles for the reaction under study or one of its crossed channel. This density matrix formalism, with a strong physics content, generalises easily to the inclusive reactions.

For the inclusive reactions, for which there is no quadratic identities, the Cauchy-Schwartz inequality is often used.

It is also interesting to interpret the inequalities as constraints on the transfer of the quantum information carried by spins from the initial to the final state. The $S$-matrix can indeed be viewed as a quantum device and the strongest positivity constraints will appear when considering entangled spin states in the direct or crossed channels.

Outline The growing number and variety of polarised experiments calls for a permanent effort on the theoretical side towards the unification and simplification of the spin formalism, using physically intuitive notations. Powerful methods are also required to derive the model-independent constraints (equalities and inequalities). As an update of the previous reviews $[1,2,48]$ the main aim of this report is to present these constraints, first in a general unified manner, then for typical particular reactions. Inequalities are in fact the major topic of this review. Some interpretation in terms of quantum information will be proposed. The analogy between the low- or high-energy hadron physics and the physics of structure functions and parton distributions will be stressed.

For each chosen reaction, the positivity constraints will be compared with the recent data and a short review the underlying dynamics will be given: meson exchange, internal quark-antiquark annihilation, QCD evolution, etc. This article is by no means a comprehensive summary of the data on spin measurements and their interpretation. Instead, the examples have been chosen in lowenergy hadronic reactions and in the physics of parton distribution to illustrate the role of positivity constraints. The selection is rather arbitrary, and due to lack of space, entire chapters are omitted, such as hadron form factors.

This review is organised as follows. In the next section, we briefly recall the basic formalism of spin observables, spin amplitudes, density matrix, etc., and the role of symmetries. General methods for deriving the positivity constraints will be presented. In Sec. 3, we review the case of some exclusive reactions, such as $\pi \mathrm{N} \rightarrow \pi \mathrm{N}, \overline{\mathrm{N}} \mathrm{N} \rightarrow \pi \pi$, the photoproduction of pseudoscalar or vector particles and the hyperon-pair production $\bar{p} p \rightarrow \bar{\Lambda} \Lambda$, for which various spin observables can be measured. Inequalities constraining the spin observables are derived and compared to some available experimental data.

This study is resumed in Sec. 4 for the inclusive reactions. It includes spin observables for the hadronic inclusive reactions, structure functions and parton distributions.

In Sec. 5, we briefly present the aspects related to quantum information, and the ways of adapting the cascade simulations to account for the spin degrees of freedom.

We consider the future of this physics in Sec. 6, with a discussion of the forthcoming facilities, detectors, polarised beams and targets. 


\section{Basic formalism}

In this section, the notation is introduced for kinematical variables and experimental observables. This is a minimal reminder. A more comprehensive formalism is available in previous review articles and textbooks $[1,2,49]$. In particular, the delicate issue of translating the measurements from the laboratory frame to the centre-of-mass frame is omitted here.

Each particular reaction has its own tradition, that we shall try to follow, at the expense of a slight modification of the notation from one section to another.

\subsection{Spin observables}

\subsubsection{Kinematics and frames}

Let us consider a reaction $a+b \rightarrow c+d$, with four-momenta $\tilde{p}_{a}, \ldots \tilde{p}_{d}$, and Mandelstam variables

$$
s=\left(\tilde{p}_{a}+\tilde{p}_{b}\right)^{2}, \quad t=\left(\tilde{p}_{a}-\tilde{p}_{c}\right)^{2}, \quad u=\left(\tilde{p}_{a}-\tilde{p}_{d}\right)^{2} .
$$

In the centre-of-mass frame, the three-momenta are $\boldsymbol{p}=\boldsymbol{p}_{a}=-\boldsymbol{p}_{b}$ and $\boldsymbol{p}^{\prime}=\boldsymbol{p}_{c}=-\boldsymbol{p}_{d}$. The particles are ordered as follows: $a$ is the beam, $b$ the target, $c$ the scattered particle, and $d$ the recoiling one, so that for elastic scattering $c$ is identical to $a$, and $d$ to $b$. Except in the case of extreme forward or backward scattering, the scattering plane is well defined, with a normal vector $\boldsymbol{n}=\boldsymbol{p} \times \boldsymbol{p}^{\prime} /\left|\boldsymbol{p} \times \boldsymbol{p}^{\prime}\right|$. A frame is attached to each particle to project out its spin components,

$$
\begin{array}{ll}
\left\{\boldsymbol{l}_{a}=\hat{\boldsymbol{p}}, \boldsymbol{m}_{a}, \boldsymbol{n}\right\}, & \left\{\boldsymbol{l}_{b}=-\hat{\boldsymbol{p}}, \boldsymbol{m}_{b}, \boldsymbol{n}\right\}, \\
\left\{\boldsymbol{l}_{c}=\hat{\boldsymbol{p}}^{\prime}, \boldsymbol{m}_{c}, \boldsymbol{n}\right\}, & \left\{\boldsymbol{l}_{d}=-\hat{\boldsymbol{p}}^{\prime}, \boldsymbol{m}_{d}, \boldsymbol{n}\right\},
\end{array}
$$

as illustrated in Fig. 1, but the index of the particle is often omitted. In each case, the sideways axis is defined as $\boldsymbol{m}_{i}=\boldsymbol{n} \times \boldsymbol{l}_{i}$. The components $\left\{V_{m}, V_{n}, V_{l}\right\}$ of a vector are sometimes denoted $\left\{V_{x}, V_{y}, V_{z}\right\}$. Another set of axes, $\{\boldsymbol{S}, \boldsymbol{N}, \boldsymbol{L}\}$ is used in the literature, following the so-called Argonne convention [50]. It coincides with $\{\boldsymbol{m}, \boldsymbol{n}, \boldsymbol{l}\}$ except for particle $b$ where $\{\boldsymbol{S}, \boldsymbol{N}, \boldsymbol{L}\}=\{-\boldsymbol{m}, \boldsymbol{n},-\boldsymbol{l}\}$.

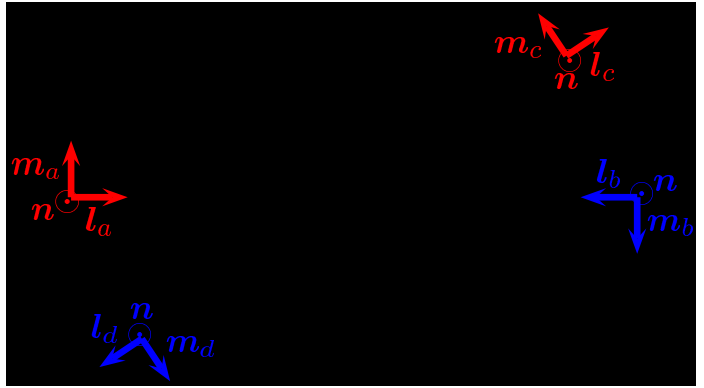

Figure 1: Axes to project out the spin components of each particle in the centre-of-mass frame of the reaction $a+b \rightarrow c+d$.

\subsubsection{Definition of the spin observables}

Some of the results below hold without limitation. For others, it is implicitly assumed that the spin is either 0 or $1 / 2$, the case of photons or massive vector particles requiring some specific treatment.

For a reaction $a+b \rightarrow c+d$ involving spinless particles, the only observable is the angular distribution $I_{0}$, sometimes called differential cross-section. It is a function of the centre-of-mass energy $\sqrt{s}$ and scattering angle $\theta$. 


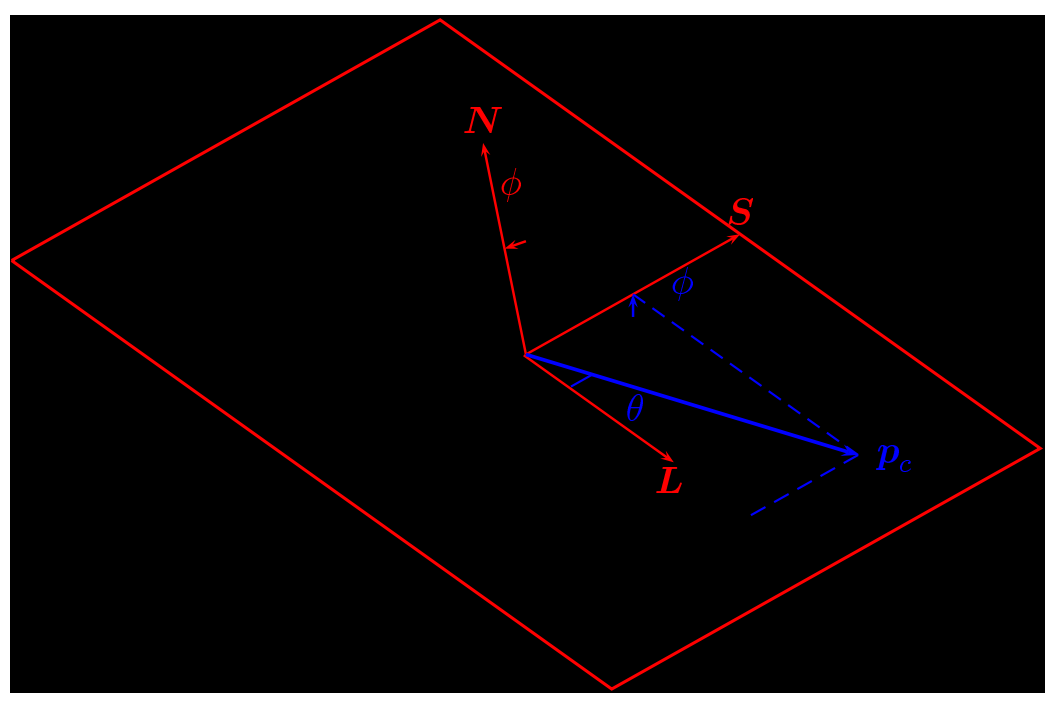

Figure 2: The scattering plane is rotated by $\phi$ with respect to the frame defined by the target polarisation and the beam axis.

If the particle $b$ has spin, the target may be polarised. A longitudinal polarisation $S_{z}$ does not affect the azimuthal distribution of the scattered particle $c$ or recoil particle $d$. However, a transverse polarisation $S_{T}$ gives an azimuthal asymmetry measured by the analysing power $A_{n}$. In a coordinate frame attached to $\boldsymbol{S}_{T}$, the differential cross section is

$$
I(s, t)=\frac{\mathrm{d} \sigma}{\mathrm{d} \Omega}=I_{0}(s, t)\left(1+A_{n}(s, t) \boldsymbol{S}_{T} . \boldsymbol{n}\right)=I_{0}\left(1+A_{n} S_{T} \cos \phi\right),
$$

where $I_{0}$ is the differential cross-section in absence of polarisation and the azimuthal angle $\phi$ of the scattered particle is equal to the angle between $\boldsymbol{S}_{T}$ and $\boldsymbol{n}$ (see Fig. 2). In some of the more systematic notation schemes, the analysing power is denoted $C_{0 y 00}$, or $(0 y \mid 00)$. The positivity of $I$ imposes $\left|A_{n}\right| \leq 1$.

Similarly, if the particle $a$ has spin, the beam can be polarised. A beam asymmetry parameter can be defined by an equation analogous to (2.3). For the case of a photon beam, see Sec. 3.4 on photoproduction.

If both the beam and the target are polarised, the cross section can be written in the most general form

$$
I\left(\boldsymbol{S}_{a}, \boldsymbol{S}_{b}\right)=I_{0} C_{\lambda \mu 00} S_{a}^{\lambda} S_{b}^{\mu}, \quad C_{0000}=S_{a}^{0}=S_{b}^{0}=1 .
$$

We have introduced the four-component polarisation vector $S^{\mu}=\left(S^{0}, \boldsymbol{S}\right)$ where $\boldsymbol{S}=\langle\boldsymbol{\sigma}\rangle$ is the usual polarisation vector, with $|\boldsymbol{S}| \leq S^{0}=1$. The Greek indices take the values $0, m, l, n$, or $0, x, y, z$, whereas the Latin indices $i, j$ take only the values $m, l, n$, or $x, y, z$. A summation is implied over each repeated index. The components $S^{x}, S^{y}$ and $S^{z}$ are measured in the frame (2.2) associated to each particle. The azimuthal dependence of the differential cross section, like in Eq. (2.3), is then due to the variations of $S^{x}$ and $S^{y}$ when the scattering plane is rotated about the beam axis, $S$ keeping a fixed direction in the laboratory frame.

$S^{\mu}$ is not the relativistic polarisation 4 -vector $\mathrm{s}^{\mu}$ which appears in covariant expressions such as (4.35). For a particle of 4-momentum $(\gamma m, 0,0, \gamma v m)$ moving along the $\boldsymbol{z}$ axis, the correspondence is

$$
\left(\mathrm{s}^{0}, \mathrm{~s}^{x}, \mathrm{~s}^{y}, \mathrm{~s}^{z}\right)=\left(\gamma v S^{z}, S^{x}, S^{y}, \gamma S^{z}\right) .
$$

In the rest frame, the $x, y$ and $z$ components are the same, but $\mathrm{s}^{0}=0$ while $S^{0} \equiv 1$.

Equation (2.4) applies whether or not discrete symmetries like $P$ and $C$ are conserved. If parity is conserved, the non-vanishing spin parameters are the beam asymmetry $C_{n 000}$, the analysing power 
$C_{0 n 00}$ and the initial-state double-spin asymmetry coefficients ${ }^{2} C_{i j 00}$, with $i j=x x, x z, z x, z z$ and $y y$.

If a final-state particle $c$ or $d$ has a spin, its average value can be measured. In the case of unpolarised beam and target, this defines the polarisation, $P_{n}^{c}$ or $P_{n}^{d}$, or, in a more systematic notation, $C_{00 n 0}$ or $C_{000 n}$. Parity conservation requires, indeed, that this polarisation is normal to the scattering plane. If both final polarisations are measured, the final-state spin correlation coefficients $C_{00 i j}$ can be determined. For the most general case where both the beam and the target are polarised, and the final spins analysed, the analogue of (2.4) reads

$$
I\left(\boldsymbol{S}_{a}, \boldsymbol{S}_{b}, \boldsymbol{S}_{c}, \boldsymbol{S}_{d}\right)=I_{0} C_{\lambda \mu \nu \tau} S_{a}^{\lambda} S_{b}^{\mu} S_{c}^{\nu} S_{d}^{\tau} .
$$

The quantities $C_{\lambda \mu \nu \tau}$ are called the Cartesian reaction parameters. A variety of alternative notations can be found in the literature, such as $(\lambda \mu \mid \nu \tau)$ also used in this report.

In Eq. (2.6), $\boldsymbol{S}_{c}$ and $\boldsymbol{S}_{d}$ are chosen (for instance by a selective detector) spin orientations of the final particles. They are input variables, not depending on $\boldsymbol{S}_{a}, \boldsymbol{S}_{b}$ nor on the reaction mechanism. They have to be distinguished from the (output) polarisations of these particles, that we denote $\left\langle\boldsymbol{S}_{c}\right\rangle$ and $\left\langle\boldsymbol{S}_{c}\right\rangle$ (we have have kept the same letters for simplicity, but with an acute bracket). The latter depend on $\boldsymbol{S}_{a}, \boldsymbol{S}_{b}$ and the reaction. For particle $c$, we have

$$
\left\langle\boldsymbol{S}_{c}\right\rangle=\frac{\nabla_{\boldsymbol{S}_{c}} I\left(\boldsymbol{S}_{a}, \boldsymbol{S}_{b}, \boldsymbol{S}_{c}, \boldsymbol{S}_{d}=0\right)}{I\left(\boldsymbol{S}_{a}, \boldsymbol{S}_{b}, \boldsymbol{S}_{c}=0, \boldsymbol{S}_{d}=0\right)},
$$

where $\nabla$ stands for the usual gradient operator, and similarly for $d$.

\subsubsection{Centre-of-mass and other frames}

Along this review, we restrict ourselves to the centre-of-mass frame, where most experimental results are given, and where the constraints of symmetries such as identical particles or time reversal are most easily expressed.

It is in general rather delicate to translate the spin observables from one frame to another, for instance from the target frame to the centre-of-mass one. See, e.g., [1,2]. However, it could be checked that many of the identities or inequalities survive the various rotations associated to a change of frame. This is the case, for instance, for the identity $A^{2}+R^{2}+D^{2}=1$ of $\pi \mathrm{N}$ scattering, as discussed in Sec. 3.1.

\subsection{General properties of density matrices}

The density matrix will be used extensively to derive constraints on spin observables. Here, the properties associated with its rank and positivity are briefly reminded.

\subsubsection{Definitions}

The density matrix $\rho$ of a quantum system, e.g., the spin state of one or several particles, describes its statistical properties and encodes the relevant information. It has dimension $N \times N$, where $N$ is the dimension of the quantum Hilbert space of the system, in our case the number of basic spin states: $2 s+1$ for one massive particle of spin $s,\left(2 s_{a}+1\right)\left(2 s_{b}+1\right)$ for two massive particles $a$ and $b$, etc. The expectation value of the observable associated to the operator $\mathcal{O}$ is

$$
\langle\mathcal{O}\rangle=\operatorname{Tr}\{\rho \mathcal{O}\} .
$$

The prototype of a matrix density is that of one spin $1 / 2$ particle, which is written as

$$
\rho=(\mathbb{1}+\boldsymbol{S} . \boldsymbol{\sigma}) / 2, \quad|\boldsymbol{S}| \leq 1,
$$

\footnotetext{
${ }^{2}$ Note that the $C_{i j 00}$, often abbreviated as $C_{i j}$ are sometimes named "correlation coefficients", though there are not, strictly speaking, defined as correlation factors in statistics.
} 
where $\boldsymbol{S}$ is the polarisation vector, $\mathbb{1}$ the $2 \times 2$ unit matrix and $\boldsymbol{\sigma}=\left\{\sigma_{x}, \sigma_{y}, \sigma_{z}\right\}$ the set of Pauli matrices.

If the system is known to be in a well-defined quantum state $|\psi\rangle$, also called a pure state, $\rho$ is the rank-one projector $\rho=|\psi\rangle\langle\psi|$. If not, the system is in a mixed state and $\rho$ can be decomposed as follows

$$
\rho=\sum_{n} w_{n}\left|\psi_{n}\right\rangle\left\langle\psi_{n}\right|, \quad 0 \leq w_{n} \leq 1, \quad \operatorname{Tr} \rho=\sum_{n} w_{n}=1 .
$$

The set of $\left|\psi_{n}\right\rangle$ in Eq. (2.10) is not unique. Even the number of terms can be varied for a given $\rho$. However $\rho$ is Hermitian, hence diagonalisable, and one obtains a minimal number of terms by taking the $w_{n}$ and the $\left|\psi_{n}\right\rangle$ to be the eigenvalues and eigenvectors of $\rho$. These $\left|\psi_{n}\right\rangle$ form an orthonormal basis and $w_{n}$ is the probability that the system is in the state $\left|\psi_{n}\right\rangle$. From now on $w_{n}$ will be the $n^{\text {th }}$ eigenvalue of $\rho$.

The number $r \leq N$ of non-vanishing $w_{n}$ is the rank of $\rho$. The rank is one type of entropy: the smaller it is, the larger is the information on the system. The rank-one projector considered above contains the maximum information. This is the case of a fully polarised particle. Other types of entropy are presented in Sec. 5. The minimum information is given by $\rho=\mathbb{1}_{N} / N$, where $\mathbb{1}_{N}$ is the $N \times N$ unit matrix. It is the case of a completely unpolarised particle.

Objects similar to density matrices, but of arbitrary positive trace, describe spin-dependent probabilities, in particular

- the acceptance matrix of a detector, which can be written as $\check{\rho}=\sum_{n} w_{n}\left|\psi_{n}\right\rangle\left\langle\psi_{n}\right|$, where $w_{n}$ is the probability of registering the particle if it is in the spin state $\left|\psi_{n}\right\rangle$ (the $\left|\psi_{n}\right\rangle$ form an orthogonal basis). Then $0 \leq w_{n} \leq 1$ but there is no imposed constraint on the trace. For a full-efficiency non-analysing detector, $\check{\rho}$ is the identity matrix.

- the non-isotropic decay of an unstable polarised particle can be described by a decay matrix $\check{\rho}\left(\boldsymbol{p}_{1}, \boldsymbol{p}_{2}, \ldots\right)$ which depend on the momenta $\boldsymbol{p}_{1}, \boldsymbol{p}_{2}, \ldots$ of the decay products. It has the same properties as the acceptance matrix of a detector. Such a matrix can also be introduced for the fragmentation of a polarised quark or gluon.

- the Cross Section Matrix (CSM) $\mathcal{R}$, which encodes all the spin dependence of a reaction. Its matrix elements are linear functions of the cartesian parameters $C_{\lambda \mu \nu \tau}$ and its trace is proportional to the unpolarised cross section. It acts on quantum states containing the initial and the final particles together. It will be introduced in Sec. 2.5.1 and used extensively for deriving general rules and studying particular reactions.

\subsubsection{The positivity conditions}

The density matrix $\rho$, as well as the similar objects $\check{\rho}$ and $\mathcal{R}$ mentioned above, are semi-positive: $\langle\psi|\rho| \psi\rangle \geq 0$ for any state $|\psi\rangle$. We shall write it $\rho \geq 0$. If $\langle\psi|\rho| \psi\rangle>0$ for all states, the matrix is said to be positive or positive definite $(\rho>0)$. The positivity of $\rho$ is equivalent to the positivity of all the $w_{n}$ 's. For semi-positivity, some eigenvalues are allowed to be zero. $\rho \geq 0$ implies $(\operatorname{Tr} \rho) \mathbb{1}_{N}-\rho \geq 0$, where $\mathbb{1}_{N}$ is the unit $N \times N$ matrix. For an acceptance matrix we have the independent constraint $\mathbb{1}_{N}-\rho \geq 0$, owing to $w_{n} \leq 1$.

Let us consider the symmetric functions of the eigenvalues of an Hermitian matrix $\rho$

$$
\Delta_{1}=\sum_{i} w_{i}, \quad \Delta_{2}=\sum_{i<j} w_{i} w_{j}, \quad \Delta_{3}=\sum_{i<j<k} w_{i} w_{j} w_{k}, \quad \cdots \quad \Delta_{N}=w_{1} w_{2} \cdots w_{n} .
$$

A necessary and sufficient condition for $\rho$ to be semi-positive and of rank $r$ is

$$
\Delta_{p}>0 \text { for } p \leq r, \quad \Delta_{p}=0 \text { for } r<p \leq N .
$$

These conditions can be expressed in terms of determinants. A minor is any subdeterminant obtained by removing some rows and the same number of columns. In a principal minor the removed rows 
and columns have the same indices, so that the surviving diagonal elements remain diagonal. $\Delta_{p}$ is the sum of the principal minors of order $p$. In particular, $\Delta_{1}=\operatorname{Tr}(\rho), \Delta_{N}=\operatorname{det}(\rho)$.

Calculating all $\Delta_{p}$ 's is tedious since there are $N ! /[p !(N-p) !]$ principal minors of size $p \times p$ for each $p$, in total $2^{N}-1$ determinants. However, deciding whether or not a matrix is (semi-) positive requires much fewer determinants:

1. If $\rho$ is positive definite, each principal minor is strictly positive. If $\rho$ is semi-positive, each principal minor is zero or positive. This gives inequalities generally simpler than (2.12). If anyone principal minor is negative, one can conclude that $\rho$ is non-positive.

2. If a nested sequence of $N$ principal minors is strictly positive, $\rho$ is positive definite. "Nested" means that the $p^{\text {th }}$ determinant is a principal minor of the $p+1^{\text {th }}$ one. One example of nested sequence is the set of "corner principal minors", the $p^{\text {th }}$ one occupying the $p$ first rows and columns of $\rho$.

3. If a nested sequence of $N$ principal minors is strictly positive except for the largest one which is zero, $\rho$ is semi-positive of rank $N-1$.

The most used positivity conditions result from applying item 1) to the diagonal elements and the $2 \times 2$ principal minors:

$$
\rho_{k k} \geq 0, \quad\left|\rho_{i j}\right|^{2} \leq \rho_{i i} \rho_{j j} \quad \text { (without index summation) }
$$

which, combined with $\operatorname{Tr} \rho=1$, imply $\left|\rho_{i j}\right| \leq 1$ (more generally, $\left|\rho_{i j}\right| \leq \operatorname{Tr} \rho$ ).

Item 2) cannot be extended to semi-positivity. As a counter-example, the matrix

$$
\rho=\left(\begin{array}{lll}
1 & 1 & 1 \\
1 & 1 & 1 \\
1 & 1 & a
\end{array}\right),
$$

has its three "corner principal minors" positive or zero, but $\rho$ is non-positive for $a<1$.

\subsubsection{The full domain of positivity}

Let us release provisionally the trace condition. An Hermitian matrix $\rho$ depends linearly on $N^{2}$ real parameters, for instance $\Re \mathrm{e}\left(\rho_{i i^{\prime}}\right)$ for $i \leq i^{\prime}$ and $\Im \mathrm{m}\left(\rho_{i i^{\prime}}\right)$ for $i<i^{\prime}$. In the $N^{2}$-dimensional parameter space, the domain where $\rho$ is semi-positive is a convex half-cone $\mathcal{C}$ : if $\rho$ belongs to $\mathcal{C}$, then $a \rho$ also belongs to $\mathcal{C}$ for $a \geq 0$. If $\rho_{1}$ and $\rho_{2}$ belong to $\mathcal{C}$, then $x \rho_{1}+(1-x) \rho_{2}$ also belongs to $\mathcal{C}$ for $0 \leq x \leq 1$ (convexity). Indeed, for any $|\psi\rangle$ we have $\left\langle\psi\left|\rho_{1}\right| \psi\right\rangle \geq 0$ and $\left\langle\psi\left|\rho_{2}\right| \psi\right\rangle \geq 0$, hence $\left\langle\psi\left|x \rho_{1}+(1-x) \rho_{2}\right| \psi\right\rangle \geq 0$. We can call $\mathcal{C}$ the positivity cone.

The space of Hermitian matrices is endowed by a Euclidian metric. The norm of a matrix can be defined as $|\rho|=\sqrt{\operatorname{Tr} \rho^{2}}$, the distance between two matrices $\rho$ and $\rho^{\prime}$ is $\left|\rho-\rho^{\prime}\right|$ and their scalar product is $\left(\rho, \rho^{\prime}\right)=\operatorname{Tr}\left(\rho \rho^{\prime}\right)$. One can decompose $\rho$ as follows

$$
\rho=\rho_{\|}+\rho_{\perp}, \quad \rho_{\|}=(\operatorname{Tr} \rho / N) \mathbb{1}, \quad \operatorname{Tr} \rho_{\perp}=0 .
$$

$\rho_{\perp}$ carries the information and $\left|\rho_{\perp}\right|^{2}$ is a kind of negentropy ${ }^{3}$. One has

$$
|\rho|^{2}=\left|\rho_{\perp}\right|^{2}+\left|\rho_{\|}\right|^{2}, \quad\left|\rho_{\|}\right|=\operatorname{Tr} \rho / \sqrt{N}, \quad 2 \Delta_{2}=(N-1)\left|\rho_{\|}\right|^{2}-\left|\rho_{\perp}\right|^{2} .
$$

For $N=2$, one can parametrise $\rho$ as $\left(S^{0} \mathbb{1}+\boldsymbol{S} . \boldsymbol{\sigma}\right) / 2$, where $\left(S^{0}, \boldsymbol{S}\right)$ generalises the 4-vector $S^{\mu}$ introduced in (2.4) for arbitrary $S^{0}=\operatorname{Tr} \rho$. The positivity cone is simply given by $S^{0} \geq 0$, $4 \Delta_{2}=\left(S^{0}\right)^{2}-S^{2} \geq 0$. It reminds the future cone in the usual Minkowski space.

\footnotetext{
${ }^{3}$ Negative of entropy
} 
Let us now impose the trace condition $\operatorname{Tr} \rho=1$ which amounts to take the intersection of $\mathcal{C}$ with the hyperplane $P$ of equation $\operatorname{Tr}(\rho)=1$. It gives a finite and convex positivity domain $\mathcal{D}$, as represented in Fig. 3. Convexity follows from the same argument as for $\mathcal{C}$. Finiteness is guaranteed by $\left|\rho_{i j}\right| \leq 1$ for each matrix element. The centre of the domain is the matrix $\rho_{\|}=\mathbf{1}_{N} / N$, for which $\rho_{\perp}=0$. A similar positivity domain exists for the normalised cross section matrix $\hat{\mathcal{R}}=(N / \operatorname{Tr} \mathcal{R}) \mathcal{R}$.

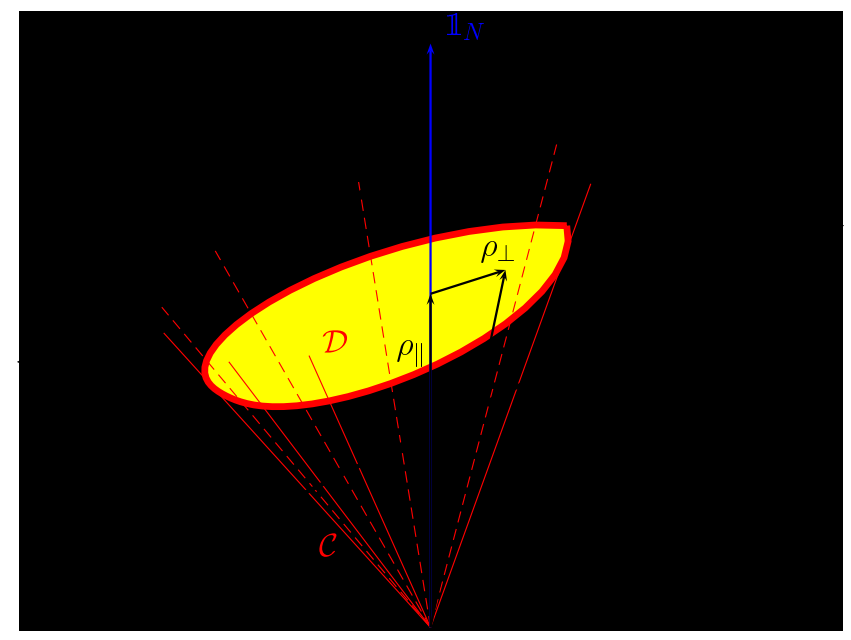

Figure 3: Positivity cone $\mathcal{C}$ for matrices of arbitrary trace and positivity domain $\mathcal{D}$ for unit-trace matrices. $P$ is the hyperplane $\operatorname{Tr}(\rho)=1$. The decomposition $\rho=\rho_{\|}+\rho_{\perp}$ of Eq. (2.15) is also schematically represented.

The boundary $\partial \mathcal{D}$ of $\mathcal{D}$ is a sheet of the hypersurface $\Delta_{N} \equiv \operatorname{det}(\rho)=0$. It is a manifold of dimension $\left(N^{2}-2\right)$ and degree $N$. On $\partial \mathcal{D}$, $\rho$ is only semi-positive. The other conditions, $\Delta_{p} \geq 0$ for $p=2, \cdots N-1$ define domains which include $\mathcal{D}$. These "auxiliary" conditions serve to eliminate unphysical sheets of the hypersurface $\Delta_{N}=0$. The hypersurface where $\Delta_{p}$ (or any principal minor) vanishes is externally tangent to $\mathcal{D}$. The contact zone contains the semi-positive matrices of rank $r<p$.

The distance to $\partial \mathcal{D}$, which grows with $\Delta_{N}$, is a form of entropy. This is for instance the case of the cross section matrix of an inclusive reaction, where information is lost through non-detected particles.

Case of rank-one matrices. The density matrices we shall encounter are often of rank $r$ smaller than their dimension $N$, therefore sit on $\partial \mathcal{D}$. The density matrix $\rho=|\psi\rangle\langle\psi|$ of a pure state has $r=1$. In this case, $\Delta_{p}=0$ for all $p \geq 2$, and all non-linear positivity constraints are saturated. According to (2.16), such matrices have the largest distance $d_{\max }=[(N-1) / N]^{1 / 2}$ from the centre. One can say that they are at the "corners" of $\mathcal{D}$. The same properties apply to the cross section matrix of a completely polarised exclusive reaction, which also has $r=1$. For $r>1, \Delta_{2}$ is positive; it is another form of entropy.

Symmetries of the positivity domain. The (semi-) positivity of a matrix $\rho$ is conserved under a unitary transformation $\rho \rightarrow U \rho U^{\dagger}$ and under the transposition $\rho \rightarrow \rho^{t}$. Accordingly, $\mathcal{D}$ is invariant under such transformations. They conserve $\rho_{\|},|\rho|$ and $\left|\rho_{\perp}\right|$, therefore $U$ can be considered as a sort of rotation of $\rho_{\perp}$ whereas transposition is a reflection about the hyperplane of real matrices. Using unitary transformations, one obtains an infinity of other symmetry hyperplanes.

Let us however point out that for $N>2$, $\mathcal{D}$ is not symmetrical about its centre. Opposite to a pure state matrix $\rho=|\psi\rangle\langle\psi|$ with $\left|\rho_{\perp}\right|=d_{\max }$ one has another boundary matrix $\rho^{\prime}=(\mathbb{1}-$ $|\psi\rangle\langle\psi|) /(N-1)$ at distance $\left|\rho_{\perp}^{\prime}\right|=d_{\max } /(N-1)$ from the centre. 
Symmetries such as parity, charge conjugation, time reversal, etc. studied in Sec. 2.8 impose linear or anti-linear relations to $\rho$, for instance $\rho=P \rho P^{-1}$ for parity. These relations together define a hyperplane $H$ which is a symmetry plane of the unrestricted $\mathcal{D}$. The effective domain for $\rho_{\perp}$ is then the intersection $\mathcal{D}_{\text {eff }}=\mathcal{D} \cap H$.

\subsubsection{Structure of the boundary of $\mathcal{D}$}

Except for the case $N=2$ the boundary of $\mathcal{D}$ has a complex structure which is difficult to represent by a drawing and barely compares with familiar three-dimensional bodies. The bulk of $\partial \mathcal{D}$ is made by matrices of rank $N-1$. Decreasing the rank unit by unit, one obtains a hierarchy of "edges" of lower and lower dimensions:

$$
\mathcal{D} \equiv \mathcal{D}_{N}^{N} \supset \mathcal{D}_{N-1}^{N} \supset \ldots \supset \mathcal{D}_{2}^{N} \supset \mathcal{D}_{1}^{N} .
$$

The first domain of this chain is $\mathcal{D}$ itself. $\mathcal{D}_{N-1}^{N}=\partial \mathcal{D}$ is the whole boundary. $\mathcal{D}_{r}^{N}$ is the set of semi-positive density matrices of rank less than or equal to $r$, characterized by $\Delta_{r+1}=0$ (which also insures $\Delta_{r^{\prime}}=0$ and the vanishing of all $r^{\prime} \times r^{\prime}$ subdeterminants for any $r^{\prime} \geq r$ ). $\mathcal{D}_{1}^{N}$, which contains the pure state density matrices, is the "corner" of $\mathcal{D}$.

To each semi-positive density matrix $\rho$ of rank $r$ one associates the image Hilbert space $\mathcal{H}_{r}(\rho)=$ $\rho \mathcal{H}_{N}$, of dimension $r$. On this subspace $\rho$ is strictly positive-definite. $\rho$ can thus be defined by the $r 2-1$ real parameters of its restriction to $\mathcal{H}_{r}(\rho)$, plus $2 r(N-r)$ real angles $\theta_{i}$ specifying the orientation of $\mathcal{H}_{r}(\rho)$ in $\mathcal{H}_{N}$. Thus $\mathcal{D}_{r}^{N}$ has $r^{2}-1$ flat and $2 r(N-r)$ curved dimensions, in total $r(2 N-r)-1$ dimensions. The mixture of flat and curved dimensions survives in some of the 3-dimensional projections of Fig. 13, where conical or cylindrical pieces of boundaries can be seen.

Let us apply these results to $N=4$ for illustration. $\mathcal{D}_{4}^{4} \equiv \mathcal{D}$ has $4^{2}-1=15$ dimensions. $\mathcal{D}_{3}^{4}$ has 14 dimensions, 8 flat and 6 curved. $\mathcal{D}_{2}^{4}$ has 11 dimensions, 3 flat and 8 curved. The "corner" $\mathcal{D}_{1}^{4}$ (which contains the pure states) has only 6 curved dimensions.

The edges $\mathcal{D}_{r}^{N}$ are not convex, but connected: one can pass continuously from $\rho$ to $\rho^{\prime}$ in $\mathcal{D}_{r}^{N}$, first rotating $\mathcal{H}_{r}(\rho)$ to $\mathcal{H}_{r}\left(\rho^{\prime}\right)$ (by continuous unitary transformation in $\mathcal{H}_{N}$ ), then changing the $r^{2}-1$ parameters of the restricted density matrix. In particular, the "corner" of $\mathcal{D}$, made of the pure states, is a connected manifold. However, when symmetries such as parity, time reversal, etc. are taken into account, an edge of the effective domain $\mathcal{D}_{\text {eff }}$ may not be connex. Let us consider, for instance, the density matrix (2.9) of a spin one-half. $\mathcal{D}$ is the unit ball and the "corner" is the unit sphere, which is connex. If the source of the particle is invariant under reflection about the $x y$ plane, then $S_{x}=S_{y}=0, \mathcal{D}_{\text {eff }}$ is a diameter and its corner is made of two separate points. The corners of squares, triangles, cubes, tetrahedrons, encountered in Sec. 3 are typical cases of non-connex edges.

An important property of $\partial \mathcal{D}$ is its invariance of under polar reciprocal transform, presented below.

Reminder about the polar reciprocal transform. In the 2-dimensional Euclidian space, the polar $L=\mathcal{P}(M)$ of a point $M$ with respect to a circle $C$ is a straight line defined as follows:

- if $M$ is external to the circle, one draws the lines $M A$ and $M B$ tangent to $C$ in $A$ and $B$. Then $L$ is the whole straight line $A B$, the points $X$ of which satisfy

$$
\overrightarrow{O X} \cdot \overrightarrow{O M}=p,
$$

where $O$ is the centre and $p=R^{2}$ the radius squared of the circle.

- if $M$ is internal to the circle, $A$ and $B$ become imaginary points, but $L$ is still defined by (2.18).

Conversely, $M$ is the pole of $L$ and we equally write $M=\mathcal{P}(L)$. The reciprocal polar transform $\Gamma^{\prime}=\mathcal{P}(\Gamma)$ of a curve $\Gamma$ is the envelope of $L=\mathcal{P}(M)$ when $M$ runs along $\Gamma$. This transformation 
is reciprocal: $\Gamma=\mathcal{P}\left(\Gamma^{\prime}\right)$. The correspondence between a whole straight line $L$ and its pole $M$ is a particular case of reciprocal polar transform, if $M$ is considered as a closed curve of zero length. If $\Gamma$ is a convex polygon $C_{1}, C_{2}, \ldots C_{n}$ surrounding $O, \Gamma^{\prime}$ is a polygon of the same type. Considering a corner $\hat{C}_{i}$ as an arc of circle of infinitely small radius, one can say that the corners of $\Gamma$ are reciprocal to the sides of $\Gamma^{\prime}$ and vice-versa:

$$
\hat{C}_{i}=\mathcal{P}\left(C_{i}^{\prime} C_{i+1}^{\prime}\right), \quad C_{i} C_{i+1}=\mathcal{P}\left(\hat{C}_{i+1}^{\prime}\right) \quad\left(C_{n+1} \equiv C_{1}\right) .
$$

In the continuous limit $n \rightarrow \infty, \Gamma$ and $\Gamma^{\prime}$ contain smooth curved parts and possibly corners and rectilinear parts, as in the example shown in Fig. 4.

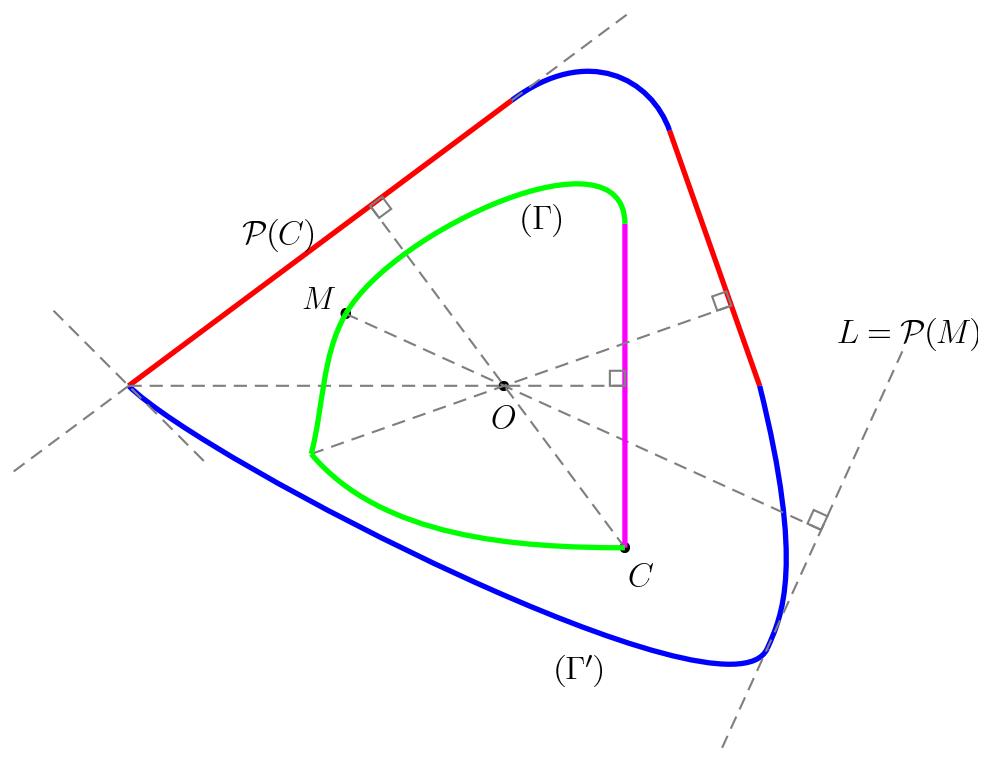

Figure 4: Schematic drawing of a 2-dimensional polar transform.

The polar transformation generalises easily to the case $p<0$ (imaginary radius) and to higher dimensions: in 3 dimensions, a plane is reciprocal to a point, a straight line is reciprocal to a straight line. In $d$ dimensions, a hyperplane of dimension $d^{\prime}$ is reciprocal to an hyperplane of dimension $d "=d-d^{\prime}-1$. The reciprocal polar transform of an ordinary curved $d-1$ dimensional surface is a surface of the same type. A corner of one curve is reciprocal to a rectilinear part of the other. Analogous correspondences for convex surfaces in $\mathbb{R}^{3}$ are:

$$
\begin{array}{lclc}
(a) & \text { cone tip } & \leftrightarrow & \text { planar face } \\
(b) & \text { trihedron tip } & \leftrightarrow & \text { planar triangular face } \\
(c) & \text { dihedron } & \leftrightarrow & \text { dihedron } \\
(d) & \text { planar curved edge } & \leftrightarrow & \text { cylindrical or conical face } \\
(e) & \text { skew edge } & \leftrightarrow & \text { "locally conical" face }
\end{array}
$$

A curved edge (here noted $\gamma$ ) of $\Gamma$ is the boundary curve between two faces, one of which at least is nonplanar. The "locally conical" surface of case (e) is the envelope of the planes reciprocal to the points of $\gamma$. The contact lines are tangent to a curve $\gamma^{\prime}$ and their reciprocal lines are tangent to $\gamma$. In case (d) $\gamma$ is in a plane and $\gamma^{\prime}$ is reduced to the pole of this plane. As an example of case (d), a cylindrical barrel is polar reciprocal to a double-cone buoy. The equator of the buoy is reciprocal to the cylindrical part of the barrel. A circular edge of the barrel is reciprocal to a cone of the buoy.

The generalisations in $\mathbb{R}^{d}$ of tips, edges, dihedrons, etc., will be called "edge" in a generic way. An edge $\mathcal{E}_{f, c}$ of $\Gamma$ having $f$ flat dimensions and $c$ curved dimensions is reciprocal to an edge $\mathcal{E}^{\prime}{ }_{f^{\prime}, c}$ of $\Gamma^{\prime}$ with the same number of curved dimensions and $f^{\prime}=d-1-c-f$. 
Application to $\mathcal{D}$. The traceless part of a density matrix $\rho$ can be represented by an Euclidean vector $\boldsymbol{\rho}_{\perp}$ of dimension $d=N^{2}-1$. For two density matrices $\rho$ and $\eta$, one has $\operatorname{Tr}(\rho \eta) \geq 0$, or

$$
\boldsymbol{\rho}_{\perp} \cdot \boldsymbol{\eta}_{\perp} \geq-1 / N .
$$

The necessary and sufficient condition that a unit trace Hermitian matrix $\eta$ belongs to $\mathcal{D}$ is that it satisfy (2.21) for any $\rho \in \mathcal{D}$. One can equally say "for any $\rho \in \partial \mathcal{D}$ ". Thus, in $\eta$ space, $\mathcal{D}$ is the intersection of the half-spaces defined by the inequality (2.21) when $\rho$ runs over $\partial \mathcal{D}$. Taking into account the fact that $\mathcal{D}$ is convex, one infers that its boundary $\partial \mathcal{D}$ is the envelope of the planes $\boldsymbol{\rho}_{\perp} . \boldsymbol{\eta}_{\perp}=-1 / N$ when $\rho$ runs over $\partial \mathcal{D}$. In other words, $\partial \mathcal{D}$ is the reciprocal transform of itself. $\partial \mathcal{D}$ is invariant under the reciprocal polar transform of power $p=-1 / N$.

$\mathcal{D}_{r}^{N}$ and $\mathcal{D}_{N-r}^{N}$ are reciprocal edges, like the objects of (2.20). Furthermore, an hyperplane tangent to $\mathcal{D}_{r}^{N}$ is reciprocal to a flat submanifold(prolongated as a whole hyperplane) of $\mathcal{D}_{N-r}^{N}$. In the example $N=4$ given earlier, $\mathcal{D}_{3}^{4}$ is reciprocal to $\mathcal{D}_{1}^{4}$ and $\mathcal{D}_{2}^{4}$ is self-reciprocal. A 6-plane tangent to $\mathcal{D}_{1}^{4}$ is reciprocal to a flat submanifold (prolongated as a whole 8-plane) of $\mathcal{D}_{3}^{4}$.

When parity, time reversal, etc. have been taken into account, the self-reciprocity property applies to $\mathcal{D}_{\text {eff }}$ as well.

\subsubsection{The projected positivity domains}

Practically one does not measure the complete set of $N^{2}-1$ parameters of the density matrix, but a restricted set of mutually orthogonal observables $\left\{\left\langle\mathcal{O}_{1}\right\rangle, \ldots\left\langle\mathcal{O}_{n}\right\rangle\right\}$ with $\operatorname{Tr}\left\{\mathcal{O}_{i} \mathcal{O}_{j}\right\}=g_{i} \delta_{i j}$ and $n<N^{2}-1$. The joint domain allowed by positivity, $\mathcal{D}\left\{\mathcal{O}_{1}, \ldots \mathcal{O}_{n}\right\}$ is obtained by successive projections of $\mathcal{D}$ on the planes $\left\langle\mathcal{O}_{k}\right\rangle=0$ with $k=n+1, n+2, \cdots N^{2}-1$. It is a convex domain, since the $\left\langle\mathcal{O}_{i}\right\rangle$ are linear functions of $\rho$.

Let us consider, for instance, the projection $\mathcal{D}\left\{\mathcal{O}_{1}, \mathcal{O}_{2}\right\}$ of $\mathcal{D}\left\{\mathcal{O}_{1}, \mathcal{O}_{2}, \mathcal{O}_{3}\right\}$ on the plane $P$ of equation $\left\langle\mathcal{O}_{3}\right\rangle=0$. It contains the intersection:

$$
\mathcal{D}\left\{\mathcal{O}_{1}, \mathcal{O}_{2}\right\} \supset P \cap \mathcal{D}\left\{\mathcal{O}_{1}, \mathcal{O}_{2}, \mathcal{O}_{3}\right\}
$$

If $P$ is a symmetry plane for $\mathcal{D}\left\{\mathcal{O}_{1}, \mathcal{O}_{2}, \mathcal{O}_{3}\right\}$, the relation $\supset$ becomes an equality. Strict inclusion is obtained for instance in Figs. 19 or 20 when the projection is made on the plane $C_{n n}=0$.

Like the distance to $\partial \mathcal{D}$, the distance to the boundary $\partial \mathcal{D}\left\{\mathcal{O}_{1}, \ldots \mathcal{O}_{n}\right\}$ of a projected domain is a kind of entropy. It can only increase under successive projections. This increase can be interpreted as the loss of information when part of the observables are integrated over.

Possible appearance of nonconvex domains. As mentioned earlier, one often deals with density matrices of rank $r$ smaller than $N$, therefore sitting on the edge $\mathcal{D}_{r}^{N}$, which is not convex. Most often the non-convexity disappears after projection on a subset of observables. For instance a hollow sphere is projected into a full disk. However this requires that the dimension of the effective domain is larger than the number of plotted quantities. A counter-example is given by the set $\left\{P_{n}, A, R\right\}$ in pion-nucleon scattering, whose domain is the hollow unit sphere (3.8). Here the cross section matrix $\mathcal{R}$ has dimension $4 \times 4$, therefore $\mathcal{D}$ has dimension 15 , but the effective domain for $\mathcal{R}$ has only 2 dimensions. It comes from $r=1$ together with parity conservation (or more simply from the fact that there are only 2 independent amplitudes).

Polar reciprocity between projection and intersection. Let $P$ be a hyperplane which contains the centre $\boldsymbol{\rho}_{\perp}=0$ of $\mathcal{D}$ and let us compare the intersection $P \cap \mathcal{D}$ and the projection $P(\mathcal{D})$ of $\mathcal{D}$ on $P$. Both domains are convex, and from the inequality (2.21) one can derive

$$
\boldsymbol{\rho}_{\perp} \cdot \boldsymbol{\eta}_{\perp} \geq-1 / N \text { for all } \rho \in P(\mathcal{D}), \eta \in P \cap \mathcal{D} .
$$

This inequality in fact defines $P(\mathcal{D})$ in terms of $P \cap \mathcal{D}$ or vice-versa. It follows that their boundaries $\partial(P \cap \mathcal{D})$ and $\partial P(\mathcal{D})$ are polar reciprocal [40]. This property applies to $\mathcal{D}_{\text {eff }}$ as well, when parity, 
time reversal, etc. have been taken into account. We will explain in Section 2.6.4 how it can be used to find $\mathcal{D}\left\{\mathcal{O}_{1}, \ldots \mathcal{O}_{n}\right\}$.

\subsection{Spin states of a single particle}

\subsubsection{Spin 1/2}

The polarisation domain for a single spin $1 / 2$ particle can be reviewed as a warm-up. A pure quantum state for a spin $1 / 2$ oriented along an axis $\boldsymbol{u}$ corresponds to an eigenstate of $\boldsymbol{\sigma} . \boldsymbol{u}$, where $\boldsymbol{\sigma}=\left\{\sigma_{x}, \sigma_{y}, \sigma_{z}\right\}$. For this state, denoted $|\boldsymbol{u}\rangle$, we have $\left\langle\sigma_{i}\right\rangle \equiv\left\langle\boldsymbol{u}\left|\sigma_{i}\right| \boldsymbol{u}\right\rangle=u_{i}$. The $\hat{\boldsymbol{z}}$ axis is usually chosen along the particle momentum (helicity basis). A state polarised toward the transverse vector $\tau$ is the following superposition of the helicity states $| \pm 1 / 2\rangle$ :

$$
|\boldsymbol{\tau}\rangle=\frac{|+1 / 2\rangle+\exp [i \phi(\boldsymbol{\tau})]|-1 / 2\rangle}{\sqrt{2}},
$$

where $\phi(\boldsymbol{\tau})$ is the azimuth of $\boldsymbol{\tau}$ in the particular frame $\left\{\hat{\boldsymbol{x}}_{\boldsymbol{p}}, \hat{\boldsymbol{y}}_{\boldsymbol{p}}, \hat{\boldsymbol{z}}_{\boldsymbol{p}}\right\}$ defined in Sec. 2.3.4. Transversity amplitudes are taken between transversity states with $\phi(\boldsymbol{\tau})= \pm \pi / 2$, i.e., $\boldsymbol{\tau}= \pm \boldsymbol{n}$ in Fig. 1.

A partially polarised state is described by the $2 \times 2$ density matrix

$$
\rho=\frac{\mathbb{1}+\boldsymbol{S} . \boldsymbol{\sigma}}{2},
$$

with expectation values

$$
\left\langle\sigma_{i}\right\rangle \equiv \operatorname{Tr}\left[\sigma_{i} \rho\right]=S_{i} .
$$

The positivity of $\rho$ is ensured by $|\boldsymbol{S}| \leq 1$. Thus the polarisation domain for a spin $1 / 2$ particle is the unit ball. A point on the ball surface corresponds to a pure quantum state. The density matrix is then the rank-one projector $\rho=|\boldsymbol{u}\rangle\langle\boldsymbol{u}|$.

The polarisation of outgoing nucleons is measured by rescattering, while for hyperons, it is deduced from the angular distribution of the decay products.

\subsubsection{Generalities on photon polarisation}

We now turn to the description of photon polarisation, on which there is a long tradition in optics. Note that "polarisation" has here a different meaning. For spin $1 / 2$, the polarisation vector is a real axial vector which is bilinear in the spinor amplitude. A longitudinally polarised electron has definite helicity. For the photon, the polarisation vector is a real or complex polar vector linear in the amplitude. There is no longitudinal polarisation, except for virtual photons. In that case, longitudinal polarisation means zero helicity, whereas helicity \pm 1 states are said to be transversely polarised.

A real photon has two possible helicity states, $|+1\rangle$ and $|-1\rangle$, corresponding to right- and left- circular polarisation, respectively (note that optics textbooks often have the opposite definition of "right" and "left"). Therefore a partial photon polarisation is described by a $2 \times 2$ density matrix analogous to that of a spin- $1 / 2$ fermion, and can be written as

$$
\rho_{\gamma}=\frac{\mathbb{1}+\boldsymbol{S} . \boldsymbol{\sigma}}{2} .
$$

$S_{1}, S_{2}$ and $S_{3}$ are the Stokes parameters of the photon, to be described shortly. The polarisation domain is again the unit ball $S_{1}^{2}+S_{2}^{2}+S_{3}^{2} \leq 1$. However the physical significance of the vector $\boldsymbol{S}$ is very different from the vector polarisation of a spin-1/2 fermion.

Another possible basis consists of two orthogonal linear polarisation states. For an exclusive or single-inclusive photon-induced reaction, they can be chosen as $|\boldsymbol{\pi}\rangle$ and $|\boldsymbol{n}\rangle$ where $\boldsymbol{n}$ is a unit vector normal to the scattering plane, $\boldsymbol{k}$ is the photon momentum and $\boldsymbol{\pi}=\boldsymbol{n} \times \boldsymbol{k} /|\boldsymbol{k}|$. These states are related to the helicity ones by

$$
| \pm 1\rangle=\frac{\mp|\boldsymbol{\pi}\rangle-i|\boldsymbol{n}\rangle}{\sqrt{2}}
$$


(the state $|+1\rangle$ differs in sign from the standard convention (2.31) for massive spin one).

A third basis is made of the two oblique states

$$
| \pm \pi / 4\rangle=\frac{|\boldsymbol{\pi}\rangle \pm|\boldsymbol{n}\rangle}{\sqrt{2}}
$$

Knowing the probabilities $p(i)$ of the photon to be in the state $|i\rangle$, and taking as basis vectors

$$
|\boldsymbol{\pi}\rangle=\left(\begin{array}{l}
1 \\
0
\end{array}\right), \quad|\boldsymbol{n}\rangle=\left(\begin{array}{l}
0 \\
1
\end{array}\right)
$$

which we call the "straight linear polarisation basis", the Stokes parameters are defined as in [51]

- $S_{3}$ or $S_{\ominus}=[p(\boldsymbol{\pi})-p(\boldsymbol{n})] /[p(\boldsymbol{\pi})+p(\boldsymbol{n})]$ (straight linear polarisation),

- $S_{1}$ or $S_{\oslash}=[p(+\pi / 4)-p(-\pi / 4)] /[p(+\pi / 4)+p(-\pi / 4)]$ (oblique linear polarisation),

- $S_{2}$ or $S_{\odot}=[p(+1)-p(-1)] /[p(+1)+p(-1)]$ (circular polarisation).

Other conventions may be used. If the helicity basis was chosen, $S_{3}$ would be the amount of circular polarisation. The straight linear polarisation basis (2.30) is particularly suitable to parityconserving reactions, in association with the transversity basis for the fermions.

\subsubsection{Massive vector particle}

Basic states A pure spin-state of a spin-1 particle (vector meson in our case) is represented by a complex 3-component column vector ${ }^{4}$. As basic states, one may choose the magnetic states $|\lambda\rangle$, eigenstates of $S_{z}(\lambda=0, \pm 1)$. For $\hat{z}$-axis along the particle momentum, $\lambda$ is the helicity. In this section, however, we will mainly use the linearly polarised (LP) basic states $\{|\hat{\boldsymbol{x}}\rangle,|\hat{\boldsymbol{y}}\rangle,|\hat{\boldsymbol{z}}\rangle\}$, also denoted $\{|1\rangle,|2\rangle,|3\rangle\}$. In what follows, generic LP states will be labelled by Latin letters $i, j, k$ or $l \in\{1,2,3\}$, and magnetic states by Greek letters $\lambda$ or $\mu$. The magnetic and LP bases are related by

$$
|\lambda=0\rangle=|\hat{\boldsymbol{z}}\rangle, \quad|\lambda= \pm 1\rangle=\mp 2^{-1 / 2}(|\hat{\boldsymbol{x}}\rangle \pm i|\hat{\boldsymbol{y}}\rangle) .
$$

For a partially polarised state, the $3 \times 3$ density matrices in the magnetic and LP bases are related by

$$
\rho_{\lambda \lambda^{\prime}}^{M}=\sum_{l l^{\prime}}\langle\lambda \mid l\rangle \rho_{l l^{\prime}}^{(L P)}\left\langle l^{\prime} \mid \lambda^{\prime}\right\rangle
$$

Expansion in basic operators. The density operator $\rho$ can be expanded on 9 basic operators $\Sigma_{\alpha}$, which are mutually orthogonal in the sense of

$$
\operatorname{Tr}\left(\Sigma_{\beta}^{\dagger} \Sigma_{\alpha}\right)=g_{\alpha} \delta_{\beta \alpha} .
$$

The squared norms $g_{\alpha}=\operatorname{Tr}\left(\Sigma_{\alpha}^{\dagger} \Sigma_{\alpha}\right)$ may be taken different from unity. Writing

$$
\rho=\sum_{\alpha} g_{\alpha}^{-1} P_{\alpha} \Sigma_{\alpha}^{\dagger}
$$

the polarisation parameters $P_{\alpha}$ are simply the expectation values of the corresponding $\Sigma_{\alpha}$ :

$$
P_{\alpha}=\left\langle\Sigma_{\alpha}\right\rangle=\operatorname{Tr}\left(\rho \Sigma_{\alpha}\right) .
$$

\footnotetext{
${ }^{4}$ In what follows, it is important to distinguish the spin state $|\phi\rangle$ from its column-vector representation in a definite basis, $\phi_{n}=\langle n \mid \phi\rangle$, and similarly the density operator $\rho$ from the density matrix, $\rho_{m n}=\langle m|\rho| n\rangle$.
} 
The $\Sigma_{\alpha}$ 's can be chosen either self-Hermitian or Hermitian conjugate by pairs (up to a sign). Then the $P_{\alpha}$ 's are either real or complex conjugate by pairs (up to a sign), and hence depend on 9 real parameters ( 8 if the condition $\operatorname{Tr} \rho=1$ is imposed). Equations (2.34-2.35) are analogous to (2.252.26), with $\sigma_{0} \equiv \mathbb{1}, P_{0} \equiv 1$.

A first explicit form of (2.34) is simply $\rho=\sum_{i, j} \rho_{i j}|i\rangle\langle j|$. Then

$$
\Sigma_{\alpha}=\Sigma_{\left\{i i^{\prime}\right\}}=\left|i^{\prime}\right\rangle\langle i|, \quad g_{\alpha}=1,
$$

where $\alpha=\left\{i i^{\prime}\right\}$ is a double index and $i, i^{\prime} \in\{x, y, z\}$. For instance, $\Sigma_{\{x y\}}=|y\rangle\langle x|,\left\langle\Sigma_{\{x y\}}\right\rangle=$ $\rho_{x y}$. This operator basis will be used in Sec. 3.5.

Cartesian parameters. Taking the linearly polarised basis, we may decompose $\rho_{i j}$ in a pure trace part $\frac{1}{3} \delta_{j k}$, a real symmetric traceless part $T_{i j}=T_{j i}$ and an imaginary anti-symmetric part,

$$
\rho=\rho_{i j}|i\rangle\left\langle j\left|=\left[\frac{1}{3} \delta_{i j}+T_{i j}-\frac{i}{2} \varepsilon_{i j k} P_{k}\right]\right| i\right\rangle\langle j|,
$$

with the constraint $T_{x x}+T_{y y}+T_{z z}=0 . \quad \varepsilon_{i j k}$ is the fully antisymmetric tensor. Accordingly, for Eq. (2.34) one may take the following operators:

$$
\begin{array}{llll}
\Sigma_{1}=\frac{|\hat{\boldsymbol{y}}\rangle\langle\hat{\boldsymbol{z}}|-| \hat{\boldsymbol{z}}\rangle\langle\hat{\boldsymbol{y}}|}{i}, & \Sigma_{2}=\frac{|\hat{\boldsymbol{z}}\rangle\langle\hat{\boldsymbol{x}}|-| \hat{\boldsymbol{x}}\rangle\langle\hat{\boldsymbol{z}}|}{i}, & \Sigma_{3}=\frac{|\hat{\boldsymbol{x}}\rangle\langle\hat{\boldsymbol{y}}|-| \hat{\boldsymbol{y}}\rangle\langle\hat{\boldsymbol{x}}|}{i}, \\
\Sigma_{4}=|\hat{\boldsymbol{y}}\rangle\langle\hat{\boldsymbol{z}}|+| \hat{\boldsymbol{z}}\rangle\langle\hat{\boldsymbol{y}}|, & \Sigma_{5}=|\hat{\boldsymbol{z}}\rangle\langle\hat{\boldsymbol{x}}|+| \hat{\boldsymbol{x}}\rangle\langle\hat{\boldsymbol{z}}|, & \Sigma_{6}=|\hat{\boldsymbol{x}}\rangle\langle\hat{\boldsymbol{y}}|+| \hat{\boldsymbol{y}}\rangle\langle\hat{\boldsymbol{x}}|, \\
\Sigma_{7}=|\hat{\boldsymbol{x}}\rangle\langle\hat{\boldsymbol{x}}|-| \hat{\boldsymbol{y}}\rangle\langle\hat{\boldsymbol{y}}|, & \Sigma_{8}=|\hat{\boldsymbol{z}}\rangle\langle\hat{\boldsymbol{z}}|-\frac{|\hat{\boldsymbol{x}}\rangle\langle\hat{\boldsymbol{x}}|+| \hat{\boldsymbol{y}}\rangle\langle\hat{\boldsymbol{y}}|}{2}, & \Sigma_{0}=\mathbb{1} .
\end{array}
$$

$\left(\Sigma_{1}, \Sigma_{2}, \Sigma_{3}\right) \equiv \boldsymbol{S}$ is the spin operator and $\left(P_{1}, P_{2}, P_{3}\right)=\boldsymbol{P}$ the associated axial polarisation. $P_{4}, \ldots P_{8}$ are the Cartesian components of the tensor polarisation, up to normalisation factors. $\left(P_{4}\right.$, $\left.P_{5}, P_{6}\right)=2 \Re \mathrm{e}\left(\rho_{y z}, \rho_{z x}, \rho_{x y}\right)$ form the non-diagonal part of it. $P_{3}, P_{6}$ and $P_{7}$, which are in $[-1,+1]$, generalise the Stokes parameters $S_{\odot}, S_{\ominus}$ and $S_{\ominus}$ to massive vector mesons moving along $\hat{\boldsymbol{z}} . P_{8} \equiv \frac{3}{2} T_{z z} \in[-1 / 2,+1]$ is the alignment parameter in the $\hat{\boldsymbol{z}}$ direction.

The operator squared norms are $g_{0}=3, g_{1}, \cdots g_{7}=2, g_{8}=\frac{3}{2}$. Apart from ordering and normalisation, $\Sigma_{1}, \ldots \Sigma_{8}$ are the Gell-Mann matrices of SU(3). In literature, the $\Sigma_{\alpha}$ for $\alpha \geq 4$ are often normalised to unit trace: $g_{\alpha}=1$. We will not do it here, to avoid square root numerical factors.

The density matrix in the linear polarisation basis reads

$$
\left(\rho_{i j}\right)=\frac{1}{2}\left(\begin{array}{ccc}
\frac{2}{3}\left(1-P_{8}\right)+P_{7} & P_{6}-i P_{3} & P_{5}+i P_{2} \\
P_{6}+i P_{3} & \frac{2}{3}\left(1-P_{8}\right)-P_{7} & P_{4}-i P_{1} \\
P_{5}-i P_{2} & P_{4}+i P_{1} & \frac{2}{3}\left(1+2 P_{8}\right)
\end{array}\right),
$$

taking the constraint $\operatorname{Tr} \rho=1$ into account.

The positivity of $\rho$, written in the form (2.39), imposes the linear, quadratic and cubic constraints

$$
\begin{aligned}
P_{1}, \ldots P_{7} \in[-1,+1], & P_{8} \in[-1 / 2,+1], \\
(4 / 9)\left(1-P_{8}\right)^{2} & \geq P_{3}^{2}+P_{6}^{2}+P_{7}^{2}, \\
(2 / 3)\left(1+2 P_{8}\right)\left[(2 / 3)\left(1-P_{8}\right)+P_{7}\right] & \geq P_{2}^{2}+P_{5}^{2}, \\
(2 / 3)\left(1+2 P_{8}\right)\left[(2 / 3)\left(1-P_{8}\right)-P_{7}\right] & \geq P_{1}^{2}+P_{4}^{2}, \\
\operatorname{det}\left(\rho_{i j}\right) & \geq 0 .
\end{aligned}
$$

The symmetric part of $\rho_{i j}$,

$$
\Theta_{i j}=\Re \mathrm{e} \rho_{i j}=\frac{1}{3} \delta_{i j}+T_{i j}
$$

is by itself positive semi-definite. One can parametrise $T_{i j}$ by two eigenvalues $t^{\prime}$ and $t^{\prime \prime}$ (the third one is $t^{\prime \prime \prime}=-t^{\prime}-t^{\prime \prime}$ ) and the three Euler angles giving the orientation of the triad of eigenvectors. The remaining parameters of $\rho$ can be the projections $\left(P^{\prime}, P^{\prime \prime}, P^{\prime \prime \prime}\right)$ of $\boldsymbol{P}$ on this triad. Positivity constrains only the five rotation-invariant quantities $\left\{t^{\prime}, t^{\prime \prime}, P^{\prime}, P^{\prime \prime}, P^{\prime \prime \prime}\right\}$. 
Spherical tensor decomposition The spherical tensor operators, which transform under the rotation group like eigenstates of $J$ and $J_{z}$, are convenient when using the magnetic basis for states. Equation (2.34) is then written in the fourth form

$$
\rho=\frac{1}{3} \mathbb{1}+\frac{1}{2} \sum_{-1}^{+1} \mathcal{P}_{\lambda} \mathcal{S}_{\lambda}^{\dagger}+\sum_{-2}^{+2} T_{2 \mu} \tau_{2 \mu}^{\dagger} .
$$

$\mathcal{P}_{\lambda}$ and $\mathcal{S}_{\lambda}$ are the magnetic components of the vector polarisation and the spin operator, which is a first-rank $(J=1)$ tensor. Using the sign convention of $(2.31), \mathcal{P}_{0}=P_{z}, \mathcal{P}_{ \pm 1}=\mp\left(P_{x} \pm i P_{y}\right) / \sqrt{2}$ and

$$
\mathcal{S}_{0}=S_{z}=\left(\begin{array}{ccc}
1 & 0 & 0 \\
0 & 0 & 0 \\
0 & 0 & -1
\end{array}\right), \quad \mathcal{S}_{-1}=\frac{S_{x}-i S_{y}}{\sqrt{2}}=\left(\begin{array}{ccc}
0 & 0 & 0 \\
1 & 0 & 0 \\
0 & 1 & 0
\end{array}\right)=-\mathcal{S}_{+1}^{\dagger} .
$$

The normalised second-rank $(J=2)$ spherical tensor operator $\tau_{2}$ are combinations of products of the $J=1$ operators:

$$
\tau_{2 \mu}=[\mathcal{S} \otimes \mathcal{S}]_{\mu}^{2}=\sum_{\lambda, \lambda^{\prime}=0, \mp 1}\left\langle 1,1 ; \lambda, \lambda^{\prime} \mid 2, \mu\right\rangle \mathcal{S}_{\lambda} \mathcal{S}_{\lambda^{\prime}},
$$

where $\left\langle 1,1 ; \lambda, \lambda^{\prime} \mid 2, \mu\right\rangle$ are the usual Clebsch-Gordan coefficients. In the magnetic basis,

$$
\tau_{20}=\frac{1}{\sqrt{6}}\left(\begin{array}{ccc}
1 & 0 & 0 \\
0 & -2 & 0 \\
0 & 0 & 1
\end{array}\right), \tau_{21}=\frac{1}{\sqrt{2}}\left(\begin{array}{ccc}
0 & -1 & 0 \\
0 & 0 & 1 \\
0 & 0 & 0
\end{array}\right)=-\tau_{2-1}^{\dagger}, \tau_{22}=\left(\begin{array}{ccc}
0 & 0 & 1 \\
0 & 0 & 0 \\
0 & 0 & 0
\end{array}\right)=\tau_{2-2}^{\dagger} .
$$

The $\tau_{2 \mu}$ operators are related to the $\Sigma_{\alpha}$ of (2.38) by

$$
\tau_{20}=-\sqrt{2 / 3} \Sigma_{8}, \quad \tau_{21}=\left(\Sigma_{5}+i \Sigma_{4}\right) / 2, \quad \tau_{22}=-\left(\Sigma_{7}+i \Sigma_{6}\right) / 2 .
$$

The same relations hold for the expectation values, $P_{\alpha}$ and $T_{2 \mu}$.

Using the form (2.42) one can write the positivity of $\rho$ in terms of the spherical parameters $\mathcal{S}_{\lambda}$ 's and the $T_{2 \mu}$ 's $[21,22,43]$. The constraints (not written here) are globally equivalent to (2.40).

Decay of a polarised vector meson in two spinless mesons. Each set of basic operators has its advantages and disadvantages. The angular distribution in the decay $V \rightarrow 2$ spin- 0 mesons is most simply expressed in terms of the tensor $\Theta_{i j}$ of (2.41). In the $V$ rest frame it reads

$$
f(\hat{\boldsymbol{k}})=\frac{\mathrm{d} N}{\mathrm{~d}^{2} \hat{\boldsymbol{k}}}=\frac{3}{4 \pi} \Theta_{i j} \hat{\boldsymbol{k}}_{i} \hat{\boldsymbol{k}}_{j},
$$

where $\boldsymbol{k}$ is the relative momentum and $\hat{\boldsymbol{k}}=\boldsymbol{k} /|\boldsymbol{k}|$. The corresponding expression with spherical tensor parameters can be found in [52]. From experimental data, one can build the moment matrix

$$
f_{m n}=\int \mathrm{d}^{2} \hat{\boldsymbol{k}} f(\hat{\boldsymbol{k}}) \hat{\boldsymbol{k}}_{m} \hat{\boldsymbol{k}}_{n}=\frac{2 \Theta_{m n}+\Theta_{i i} \delta_{m n}}{5},
$$

from which one gets

$$
\Theta_{i j}=\frac{5 f_{i j}-f_{m m} \delta_{i j}}{2} .
$$

The trace of $f_{i j}$ is equal to that of $\Theta_{i j}$, but its traceless part (related to the asphericity of $f(\hat{\boldsymbol{k}})$ ) is $2 / 5$ that of $\Theta_{i j}$. A too aspherical $f(\hat{\boldsymbol{k}})$ would violate the positivity of $\Theta_{i j}$ and could reveal an interference with other resonances or a non-resonant background.

To measure the axial polarisation $\boldsymbol{P}$ of a vector meson, at least a three-body decay is needed, in order to build an axial vector from the momenta. It works for the $\mathrm{a}_{1}$ meson [53], but not for the $\omega, \phi$ or $\mathrm{J} / \psi$ mesons. 


\subsubsection{Phase conventions for the helicity and transversity states}

It is important to define the one- and two-particle states completely, not up to a phase. Let us work in the centre-of-mass frame (the transformation to other frame will not be treated in this report). For the space coordinates one first chooses a global frame $\{\hat{\boldsymbol{x}}, \hat{\boldsymbol{y}}, \hat{\boldsymbol{z}}\}$ and introduces the primary helicity states $|p \hat{\boldsymbol{z}}, \lambda\rangle=|p \hat{\boldsymbol{z}}\rangle \otimes|\lambda\rangle$ for a particle moving along $+\hat{\boldsymbol{z}}$. For a particle of arbitrary momentum, $\boldsymbol{p}=(p, \theta, \phi)$ in polar coordinates, the helicity state $|\boldsymbol{p}, \lambda\rangle$ we take the convention of [54]:

$$
\begin{aligned}
|\boldsymbol{p}, \lambda\rangle & =R(\hat{\boldsymbol{z}} \rightarrow \hat{\boldsymbol{p}})|p \hat{\boldsymbol{z}}, \lambda\rangle \\
R(\hat{\boldsymbol{z}} \rightarrow \hat{\boldsymbol{p}}) & =\exp (-i \boldsymbol{\theta} . \boldsymbol{J})=\exp \left(-i \phi J_{z}\right) \exp \left(-i \theta J_{y}\right) \exp \left(+i \phi J_{z}\right) .
\end{aligned}
$$

$R(\hat{\boldsymbol{z}} \rightarrow \hat{\boldsymbol{p}})$ of (2.51) is the rotation of angle $\theta$ about $\boldsymbol{\theta}=\theta(\hat{\boldsymbol{z}} \times \boldsymbol{p}) /|\hat{\boldsymbol{z}} \times \boldsymbol{p}|$. It is the "minimal" rotation which transports $\hat{z}$ to $\hat{\boldsymbol{p}}$. For a spin one-half,

$$
\exp (-i \boldsymbol{\theta} . \boldsymbol{J})=(\cos \theta / 2)^{-1 / 2}(1+\boldsymbol{\sigma} \cdot \hat{\boldsymbol{p}} \boldsymbol{\sigma} \cdot \hat{\boldsymbol{z}}) .
$$

$|\boldsymbol{p}, \lambda\rangle$ defined by (2.50-2.51) is a continuous function of $\hat{\boldsymbol{p}}=\boldsymbol{p} /|\boldsymbol{p}|$ on the whole unit sphere except at the point $-\hat{z}$, where its phase is undefined, unless one fixes $\phi$ with some convention.

The components of the particle polarisation will be measured in the particular frame $\left\{\hat{\boldsymbol{x}}_{\boldsymbol{p}}, \hat{\boldsymbol{y}}_{\boldsymbol{p}}\right.$, $\left.\hat{\boldsymbol{z}}_{p}\right\}$ obtained from $\{\hat{\boldsymbol{x}}, \hat{\boldsymbol{y}}, \hat{\boldsymbol{z}}\}$ by the same rotation $R(\hat{\boldsymbol{z}} \rightarrow \hat{\boldsymbol{p}})$. This is important for the validity of formula like (2.62) or (2.66). The particular frames are also necessary to define properly the transversity and linearly polarised states which fulfil (2.24), (2.28-2.29) and (2.31). In $2 \rightarrow 2$ reactions, if we are not making the partial wave decomposition, we take $\{\hat{\boldsymbol{x}}, \hat{\boldsymbol{y}}, \hat{\boldsymbol{z}}\}=\left\{\boldsymbol{m}_{a}, \boldsymbol{n}, \boldsymbol{l}_{a}\right\}$ of Fig. 1 as global frame, and $\left\{\boldsymbol{m}_{i}, \boldsymbol{n}, \boldsymbol{l}_{i}\right\}(i=a, \ldots d)$ as particular frames.

Another convention, without the $\exp \left(+i \phi J_{z}\right)$ factor in $(2.51)$, is also used [1,2]. The resulting $|\boldsymbol{p}, \lambda\rangle$ differs from ours by the phase factor $\exp (-i \phi \lambda)$. For half-integer spin, it is double-valued or discontinuous along some line joining $+\hat{\boldsymbol{z}}$ and $-\hat{\boldsymbol{z}}$ on the unit sphere. The particular axes, called "helicity frame", are simply $\left\{\hat{\boldsymbol{x}}_{\boldsymbol{p}}, \hat{\boldsymbol{y}}_{\boldsymbol{p}}, \hat{\boldsymbol{z}}_{\boldsymbol{p}}\right\}=\left\{\boldsymbol{e}_{\theta}, \boldsymbol{e}_{\phi}, \boldsymbol{e}_{r}\right\}$, i.e., tangent to the meridian, the parallel and radial vector of the polar coordinate system at point $\hat{\boldsymbol{p}}$.

A two-particle helicity state in its centre-of-mass frame may be written as

$$
\left|\boldsymbol{k} ; a_{1}, \lambda_{1} ; a_{2}, \lambda_{2}\right\rangle=\left|a_{1}, \boldsymbol{k}, \lambda_{1}\right\rangle \otimes\left|a_{2},-\boldsymbol{k}, \lambda_{2}\right\rangle
$$

where $\boldsymbol{k}$ is the relative momentum and $a_{1}$ and $a_{2}$ are the particle types. Jacob and Wick [54] give a definition which differs from ours by the phase factor $(-1)^{s_{2}+\lambda_{2}} e^{-2 i \lambda_{2} \phi(\boldsymbol{k})}$. Their convention is convenient for the partial wave decomposition but will not be used in this report.

Application to $\boldsymbol{a}+\boldsymbol{b} \rightarrow \boldsymbol{c}+\boldsymbol{d}$. With $\{\hat{\boldsymbol{x}}, \hat{\boldsymbol{y}}, \hat{\boldsymbol{z}}\}=\left\{\boldsymbol{m}_{a}, \boldsymbol{n}, \boldsymbol{l}_{a}\right\}$ in Fig.1, we have $\left(\theta_{c}, \phi_{c}\right)=$ $\left(\theta_{\mathrm{cm}}, 0\right)$ and $\left(\theta_{d}, \phi_{d}\right)=\left(\pi-\theta_{\mathrm{cm}}, \pi\right)$. For particle $b$, we have $\theta_{b}=\pi$ and we chose $\phi_{b}=\pi$, i.e., $\boldsymbol{p}_{b, x}=-0$. For a boson, it is equivalent to $\phi_{b}=0$, but not for a fermion. The standard centre-of-mass helicity amplitude is

$$
\left\langle\gamma, \delta\left|\mathcal{M}^{a b \rightarrow c d}\left(W, \theta_{\mathrm{cm}}\right)\right| \alpha, \beta\right\rangle \equiv\left\langle\boldsymbol{p}^{\prime} ; c, \gamma ; d, \delta|\mathcal{M}| \boldsymbol{p} ; a, \alpha ; b, \beta\right\rangle \quad \text { at } \quad \phi\left(\boldsymbol{p}^{\prime}\right)=0 .
$$

The helicity amplitudes $f_{\gamma \delta \alpha \beta}$ of Jacob-Wick and $H_{\gamma \delta \alpha \beta}$ of [1,2] differ from our convention by the factors $(-1)^{s_{b}-\beta+s_{d}-\delta}$ and $e^{-i \pi(\beta-\delta)}$ respectively. In order to apply (2.62) or (2.66) they both require particular frames for $b$ and $d$ which are symmetric about $l$ from those of Fig.1, i.e., $\hat{\boldsymbol{x}}_{b, d}=$ $-\boldsymbol{m}_{b, d}, \hat{\boldsymbol{y}}_{b, d}=-\boldsymbol{n}$.

\subsection{Joint density matrix of several particles}

A two-particle system $A+B$ is described by a joint density matrix $\rho_{A B}$. The density matrix of $A$ alone, $B$ being not analysed, is given by the partial trace over $B$, denoted

$$
\rho_{A}=\operatorname{Tr}_{B} \rho_{A B}
$$


and defined by

$$
\left\langle a\left|\rho_{A}\right| a^{\prime}\right\rangle=\sum_{b}\left\langle a, b\left|\rho_{A B}\right| a^{\prime}, b\right\rangle,
$$

where $|a\rangle$ denotes a spin state of $A$. This can be generalised to more than two particles.

The spin correlations contained in $\rho_{A B}$ may be of classical nature, in which case $\rho_{A B}$ can be decomposed as

$$
\rho_{A B}=\sum_{n} w_{n} \rho_{n}(A) \otimes \rho_{n}(B) \quad \text { with } \quad w_{n} \geq 0,
$$

and is called separable. They may also be non-classical, in which case $\rho_{A B}$ cannot be written as (2.57) and is called entangled. A standard example of entangled density matrix is the spin singlet projector

$$
\rho_{A B}=\frac{\mathbb{1}-\boldsymbol{\sigma}_{A} \cdot \boldsymbol{\sigma}_{B}}{4} .
$$

This state is known to violate the Bell inequalities [55]. There are entangled states which do not violate the Bell inequalities, so that these inequalities are not a sufficient condition of separability. A more severe condition of separability is the positivity of the partially transposed matrix $\left\langle a, b\left|\rho_{A B}^{\mathrm{pt}}\right| a^{\prime}, b^{\prime}\right\rangle=\left\langle a, b^{\prime}\left|\rho_{A B}\right| a^{\prime}, b\right\rangle$ [56-58]. This condition is in fact sufficient for two spin-1/2 particles, or a system of a spin $1 / 2$ and a spin 1 .

\subsection{Description of the reactions}

Consider the reaction with polarised beam and target and final spin analysed,

$$
\vec{A}+\vec{B} \rightarrow \vec{C}+\vec{D}
$$

at fixed momenta $\boldsymbol{p}_{A}, \boldsymbol{p}_{B}, \boldsymbol{p}_{C}$ and $\boldsymbol{p}_{D}$. The scattering amplitude is written as

$$
\langle c|\otimes\langle d|\mathcal{M}| a\rangle \otimes| b\rangle \equiv\langle c, d|\mathcal{M}| a, b\rangle .
$$

Depending on the adopted basis, the letter $a$ denotes the helicity or the transversity of $A$ or, in nonrelativistic physics, $s_{z}(A)$ in a global $\{\hat{\boldsymbol{x}}, \hat{\boldsymbol{y}}, \hat{\boldsymbol{z}}\}$ frame. For a vector particle, it can also denote the direction of its linear polarisation. Squaring $\mathcal{M}$, averaging over the initial spin states and summing over the final ones give the unpolarised differential cross section

$$
\frac{d \sigma}{d \Omega}=\frac{1}{n_{A} n_{B}} \sum_{a, b, c, d}|\langle c, d|\mathcal{M}| a, b\rangle|^{2}=\frac{1}{n_{A} n_{B}} \operatorname{Tr}\left\{\mathcal{M} \mathcal{M}^{\dagger}\right\} .
$$

where $n_{p}$ is the number of possible spin states of particle $p\left(n_{p}=2 s_{p}+1\right.$ for a massive particle). The fully polarised differential cross section (2.6) reads, for spin-1/2 particles,

$$
\frac{d \sigma}{d \Omega}\left(\boldsymbol{S}_{A}, \boldsymbol{S}_{B}, \boldsymbol{S}_{C}, \boldsymbol{S}_{D}\right)=\operatorname{Tr}\left\{\mathcal{M} \frac{\mathbb{1}+\boldsymbol{S}_{A} \cdot \boldsymbol{\sigma}_{A}}{2} \otimes \frac{\mathbb{1}+\boldsymbol{S}_{B} \cdot \boldsymbol{\sigma}_{B}}{2} \mathcal{M}^{\dagger} \frac{\mathbb{1}+\boldsymbol{S}_{C} \cdot \boldsymbol{\sigma}_{C}}{2} \otimes \frac{\mathbb{1}+\boldsymbol{S}_{D} \cdot \boldsymbol{\sigma}_{D}}{2}\right\}
$$

(let us recall that the $S_{\mathrm{p}}$ and $\sigma_{\mathrm{p}}$ components for particle p are measured in the particular frame $\left\{\hat{\boldsymbol{x}}_{\mathrm{p}}, \hat{\boldsymbol{y}}_{\mathrm{p}}, \hat{\boldsymbol{z}}_{\mathrm{p}}\right\}$, which generally differs from one particle to another). More generally, the fully or partially polarised differential cross-section, for any spins, takes the form

$$
\frac{d \sigma}{d \Omega}\left(\rho_{A}, \rho_{B}, \check{\rho}_{C}, \check{\rho}_{D}\right)=\operatorname{Tr}\left\{\mathcal{M}\left[\rho_{A} \otimes \rho_{B}\right] \mathcal{M}^{\dagger}\left[\check{\rho}_{C} \otimes \check{\rho}_{D}\right]\right\} .
$$

The density matrices $\rho_{A}$ and $\rho_{B}$ of the initial particles depend on the beam and target preparation and have unit trace. If $A$ is unpolarised, $\rho_{A}=\mathbb{1}_{A} / n_{A}$. $\check{\rho}_{C}$ is not the outgoing density matrices of $C$ given by (2.65), but specifies the states accepted by the detector [59]. We call it an acceptance matrix and put an inverted hat on it. Two extreme cases are $\check{\rho}_{C}=|c\rangle\langle c|$ for an ideal detector selecting 
only state $|c\rangle$, and $\check{\rho}_{C}=\mathbb{1}_{C}$ for a non-analysing detector (or no detector at all for particle $C$ ). $\check{\rho}_{C}$ has not necessarily unit trace, but its eigenvalues cannot exceed one. The dual roles of density and acceptance matrices will be further discussed in Sec. 5 .

The joint outgoing density matrix of particle $C$ and $D$ is

$$
\rho_{C D}=\mathcal{M}\left[\rho_{A} \otimes \rho_{B}\right] \mathcal{M}^{\dagger} / \operatorname{Tr}\left\{\mathcal{M}\left[\rho_{A} \otimes \rho_{B}\right] \mathcal{M}^{\dagger}\right\} .
$$

It depends on the initial particle polarisations. The outgoing density matrix of particle $C$ alone is, according to (2.55),

$$
\rho_{C} \equiv \frac{1}{2}\left(\mathbb{1}+\left\langle\boldsymbol{S}_{C}\right\rangle \cdot \boldsymbol{\sigma}_{C}\right)=\operatorname{Tr}_{D}\left\{\mathcal{M}\left[\rho_{A} \otimes \rho_{B}\right] \mathcal{M}^{\dagger}\right\} / \operatorname{Tr}\left\{\mathcal{M}\left[\rho_{A} \otimes \rho_{B}\right] \mathcal{M}^{\dagger}\right\}
$$

where $\left\langle\boldsymbol{S}_{c}\right\rangle$ is the output polarisation of $C$ given by (2.7).

The correlation parameters entering (2.6) for a $1 / 2+1 / 2 \rightarrow 1 / 2+1 / 2$ reaction are given by

$$
C_{\lambda \mu \nu \tau}=\operatorname{Tr}\left\{\mathcal{M}\left[\sigma_{\lambda}(A) \otimes \sigma_{\mu}(B)\right] \mathcal{M}^{\dagger}\left[\sigma_{\nu}(C) \otimes \sigma_{\tau}(D)\right]\right\} / \operatorname{Tr}\left\{\mathcal{M} \mathcal{M}^{\dagger}\right\},
$$

which will be symbolically abbreviated as a sort of expectation value:

$$
(\lambda \mu \mid \nu \tau) \equiv C_{\lambda \mu \nu \tau}=\left\langle\sigma_{\lambda}(A) \sigma_{\mu}(B) \sigma_{\nu}(C) \sigma_{\tau}(D)\right\rangle,
$$

up to transpositions for $\sigma_{\nu}(C)$ and $\sigma_{\tau}(D)$, as discussed in the next section.

\subsubsection{The Cross Section Matrix}

All spin observables of reaction (2.59) can be encoded in the cross section matrix (CSM) $\mathcal{R}$, or its partial transpose $\widetilde{\mathcal{R}}$, defined by

$$
\begin{aligned}
\langle c, d|\mathcal{M}| a, b\rangle\left\langle a^{\prime}, b^{\prime}\left|\mathcal{M}^{\dagger}\right| c^{\prime}, d^{\prime}\right\rangle & =\left\langle a^{\prime}, b^{\prime} ; c^{\prime}, d^{\prime}|\mathcal{R}| a, b ; c, d\right\rangle \\
& =\left\langle a^{\prime}, b^{\prime} ; c, d|\widetilde{\mathcal{R}}| a, b ; c^{\prime}, d^{\prime}\right\rangle .
\end{aligned}
$$

The transposition linking $\widetilde{\mathcal{R}}$ to $\mathcal{R}$ concerns the final particles ${ }^{5}$. The diagonal elements of $\mathcal{R}$ or $\widetilde{\mathcal{R}}$ are the fully polarised cross sections when the particles are in the basic spin states. The knowledge of the non-diagonal elements allows a change of spin basis, thus the CSM describes the spin correlations in any directions. By construction, the $\mathcal{R}$ is semi-positive definite and of rank one. This is not generally

the case for $\widetilde{\mathcal{R}}$. On the other hand, $\widetilde{\mathcal{R}}$ respects the bra and ket assignments of variables $c, d, c^{\prime}, d^{\prime}$ in (2.68) and will sometimes lead to simpler formulas. In the following, we shall use either $\mathcal{R}$ or $\widetilde{\mathcal{R}}$.

Equations (2.61), (2.63), (2.64) and (2.66) can be rewritten as:

$$
\begin{aligned}
\frac{\mathrm{d} \sigma}{\mathrm{d} \Omega} & =\operatorname{Tr} \mathcal{R} /\left(n_{A} n_{B}\right)=\operatorname{Tr} \widetilde{\mathcal{R}} /\left(n_{A} n_{B}\right), \\
\frac{\mathrm{d} \sigma}{\mathrm{d} \Omega}\left(\rho_{A}, \rho_{B}, \check{\rho}_{C}, \check{\rho}_{D}\right) & =\operatorname{Tr}\left\{\widetilde{\mathcal{R}}\left[\rho_{A} \otimes \rho_{B} \otimes \check{\rho}_{C} \otimes \check{\rho}_{D}\right]\right\}, \\
\rho_{C D} & =\operatorname{Tr}_{A, B}\left\{\widetilde{\mathcal{R}}\left[\rho_{A} \otimes \rho_{B}\right]\right\} / \operatorname{Tr}\left\{\widetilde{\mathcal{R}}\left[\rho_{A} \otimes \rho_{B}\right]\right\}, \\
C_{\lambda \mu \nu \tau} & =\operatorname{Tr}\left\{\mathcal{R}\left[\sigma_{\lambda}(A) \otimes \sigma_{\mu}(B) \otimes \sigma_{\nu}^{t}(C) \otimes \sigma_{\tau}^{t}(D)\right]\right\} / \operatorname{Tr} \mathcal{R} \\
& =\operatorname{Tr}\left\{\widetilde{\mathcal{R}}\left[\sigma_{\lambda}(A) \otimes \sigma_{\mu}(B) \otimes \sigma_{\nu}(C) \otimes \sigma_{\tau}(D)\right]\right\} / \operatorname{Tr} \widetilde{\mathcal{R}} .
\end{aligned}
$$

The last equation can be generalised to any kind of initial and final observables as

$$
\left\langle\mathcal{O}_{i} \otimes \mathcal{O}_{f}\right\rangle=\operatorname{Tr}\left\{\widetilde{\mathcal{R}}\left(\mathcal{O}_{i} \otimes \mathcal{O}_{f}\right)\right\} / \operatorname{Tr} \widetilde{\mathcal{R}}, \quad \text { or } \quad \operatorname{Tr}\left\{\mathcal{R}\left(\mathcal{O}_{i} \otimes \mathcal{O}_{f}^{t}\right)\right\} / \operatorname{Tr} \mathcal{R} .
$$

\footnotetext{
${ }^{5}$ Alternatively, keeping the same $\widetilde{\mathcal{R}}$, one may define $\mathcal{R}$ as the full transpose of that given by (2.68). Then the partial transposition between $\widetilde{\mathcal{R}}$ and $\mathcal{R}$ would apply to the initial particles. This choice was done in Ref. [31], where $\mathcal{R}$ is called "grand density matrix".
} 
Only the second expression is a bona fide expectation value, since $\widetilde{\mathcal{R}}$ is not necessarily semi-positive.

The partial transposition from $\mathcal{R}$ to $\widetilde{\mathcal{R}}$ amounts to a $\mid$ ket $\rangle \leftrightarrow\langle$ bra $|$ exchange for the final particles, putting these in the same state as the initial particles in $\mathcal{R}$. This evokes the "crossing" operation

$$
\langle c, d|\mathcal{M}| a, b\rangle \leftrightarrow\langle\varnothing|\mathcal{M}| a, b, c, d\rangle .
$$

Although this operation is not the usual crossing (it does not reverse the charges, spins and fourmomenta of particles $C$ and $D$ ), the right-hand side of (2.71) has the same spin structure as the amplitudes of the true crossed reactions

$$
\text { (a) } A+B+\bar{C}+\bar{D} \rightarrow \varnothing, \quad \text { or } \quad(\text { b) } \varnothing \rightarrow \bar{A}+\bar{B}+C+D \text {. }
$$

(notwithstanding the fact that (a) and (b) have no physical kinematical region). Thus the CSM of (2.59) has the same structure as the density matrix of the final state in (b). The positivity of $\mathcal{R}$ can be interpreted as the positivity of $\rho_{\bar{A} \bar{B}, C D}$ in (2.72).

Using $\operatorname{Tr}\left\{\sigma_{\lambda} \sigma_{\mu}\right\}=2 \delta_{\lambda \mu}$, the fourth equation of (2.69) can be inverted as

$$
\widehat{\mathcal{R}} \equiv(N / \operatorname{Tr} \mathcal{R}) \mathcal{R}=C_{\lambda \mu \nu \tau} \sigma_{\lambda}(A) \otimes \sigma_{\mu}(B) \otimes \sigma_{\nu}^{t}(C) \otimes \sigma_{\tau}^{t}(D) .
$$

$\widehat{\mathcal{R}}$ is the normalised CSM which has the same trace as the unit matrix, i.e., $\operatorname{Tr} \widehat{\mathcal{R}}=N \equiv$ $n_{A} n_{B} n_{C} n_{D}$, so that $\widehat{\mathcal{R}}=\mathbb{1}_{N}$ when all Cartesian reaction parameters but $C_{0000}$ are vanishing. As an example, the normalised CSM of the reaction $1 / 2+0 \rightarrow 1 / 2+0$ is given in Table 1 in terms of $(\lambda \mid \mu) \equiv C_{\lambda \mu}$. The spin variables $b$ and $d$ as well as $\sigma_{\mu}(B)$ and $\sigma_{\tau}(D)$ have been removed from preceding equations. This table also describes a reduced CSM of the type (2.74) and will be repeatedly used later on.

Table 1: Normalised cross section matrix $\widehat{\mathcal{R}}$ of $1 / 2+0 \rightarrow 1 / 2+0 \quad(\operatorname{Tr} \widehat{\mathcal{R}}=4)$. Axis labels are $\{1,2,3\}=\{m, n, l\}$ in the helicity basis, $\{l, m, n\}$ in the transversity basis. $(0-3 \mid 1+i 2)$, for instance, is a condensed notation for $(0 \mid 1)+i(0 \mid 2)-(3 \mid 1)-i(3 \mid 2)$, which may be seen as $\left\langle\left(\mathbb{1}-\sigma_{3}\right) \otimes\left(\sigma_{1}+i \sigma_{2}\right)\right\rangle$, according to Eq. (2.67).

\begin{tabular}{c|cccc} 
& ++ & +- & -+ & -- \\
\hline++ & $(0+3 \mid 0+3)$ & $(0+3 \mid 1+i 2)$ & $(1-i 2 \mid 0+3)$ & $(1-i 2 \mid 1+i 2)$ \\
+- & $(0+3 \mid 1-i 2)$ & $(0+3 \mid 0-3)$ & $(1-i 2 \mid 1-i 2)$ & $(1-i 2 \mid 0-3)$ \\
-+ & $(1+i 2 \mid 0+3)$ & $(1+i 2 \mid 1+i 2)$ & $(0-3 \mid 0+3)$ & $(0-3 \mid 1+i 2)$ \\
-- & $(1+i 2 \mid 1-i 2)$ & $(1+i 2 \mid 0-3)$ & $(0-3 \mid 1-i 2)$ & $(0-3 \mid 0-3)$
\end{tabular}

\subsubsection{Reduced cross section matrices}

A reduced CSM can describe a reaction where only a subset of particles is polarised or analysed. It is obtained by taking the partial trace of the complete CSM over the initial unpolarised particles and over the final particles whose spins are not analysed. For instance,

$$
\mathcal{R}_{A, C}=n_{B}^{-1} \operatorname{Tr}_{B} \operatorname{Tr}_{D} \mathcal{R}_{A B, C D} .
$$

Similarly, $\mathcal{R}_{C D}$ is the trace over the initial spins, divided by $n_{A} n_{B} . \widetilde{\mathcal{R}}_{C D}=\mathcal{R}_{C D}^{t}$ is proportional to $\rho_{C D}$ of (2.64) for unpolarised $A$ and $B$.

The reduced CSM's are (semi-) positive and can be treated like the complete CSM, except for the fact that they cannot be factorised like (2.68). Therefore their ranks are generally larger than one. The reduction by a non-polarised or non-analysed particle $X$ decreases the dimension of $\mathcal{R}$ by a factor $n_{X}$ and increases its rank by the same factor $n_{X}$, up to the restriction rank $\leq$ dimension. Thus successive reductions first increase, then decrease the rank of $\mathcal{R}$. 
The CSM formalism generalises to inclusive or semi-inclusive reactions in a straightforward way: an inclusive CSM is the sum of the reduced CSM of all contributing exclusive channels, after integration over the undetected momenta. The cross section matrix $\mathcal{R}_{A B, C}$ of $A+B \rightarrow C+X$ is related by crossing to the $A+B+\bar{C} \rightarrow X$ and, by unitarity, to the discontinuity of the forward scattering amplitude of $A+B+\bar{C} \rightarrow A+B+\bar{C}$ in the variable $\left(\tilde{p}_{A}+\tilde{p}_{B}-\tilde{p}_{C}\right)^{2}$. Examples will be given later, see, e.g., Eq. (4.4), (4.18) or (4.38).

The cross section matrix is a systematic but not compulsory tool to obtain the positivity conditions. These can also be derived from Cauchy-Schwartz inequalities. This will be done, for instance in Sec. 4.3 for the Soffer inequality.

\subsubsection{Physical meaning of the positivity conditions}

The positivity of the cross section matrix of reaction (2.59) stems from the very general, but nontrivial, requirement that the probability of this reaction, as well as any reaction involving (2.59) as a sub-process, is positive. A first condition is that the cross section (2.62) is positive for arbitrary independent $\boldsymbol{S}_{A}, \boldsymbol{S}_{B}, \boldsymbol{S}_{C}$ and $\boldsymbol{S}_{D}$ in the unit ball $\boldsymbol{S}^{2} \leq 1$. Some geometrical properties of the corresponding domain are discussed in Sec. 5 and in Ref. [60]. An equivalent condition is that the polarisation of, for instance, outgoing particle $C$ for given $\boldsymbol{S}_{A}, \boldsymbol{S}_{B}$, and imposed $\boldsymbol{S}_{D}$,

$$
\left\langle\boldsymbol{S}_{C}\right\rangle=\nabla_{\boldsymbol{S}_{C}} I\left(\boldsymbol{S}_{A}, \boldsymbol{S}_{B}, \boldsymbol{S}_{C}, \boldsymbol{S}_{D}\right) / I\left(\boldsymbol{S}_{A}, \boldsymbol{S}_{B}, \boldsymbol{S}_{C}=0, \boldsymbol{S}_{D}\right)
$$

(note the difference with Eq. (2.7)) lies in the unit ball $\boldsymbol{S}_{C}^{2} \leq 1$. This condition is called classical (see Sec.5.3). As we shall see below, it is not sufficient.

Suppose, for instance, that the reduced CSM for $A$ and $B$ takes the form $\mathcal{R}_{A B} \propto \mathbb{1}+\sigma_{A}^{i} \otimes \sigma_{B}^{i}$, equivalent to $(i j \mid 00)=\delta_{i j}$. The cross section, given by the second line of (2.69) with $\check{\rho}_{C}=\mathbb{1}_{C}$, $\check{\rho}_{D}=\mathbb{1}_{D}$, is proportional to $1+\boldsymbol{S}_{A} \cdot \boldsymbol{S}_{B}$, therefore zero or positive. If $A$ and $B$ are prepared with correlated spins, one has to replace (2.69) by

$$
\frac{d \sigma}{d \Omega}\left(\rho_{A B}\right)=\operatorname{Tr}\left\{\mathcal{R}_{A B} \rho_{A B}\right\} .
$$

This is still positive for a separable $\rho_{A B}$ (see Eq. (2.57)). However for the singlet state (2.58), one obtains $d \sigma / d \Omega \propto \operatorname{Tr}\left\{\left(\mathbb{1}+\sigma_{A}^{i} \otimes \sigma_{B}^{i}\right)\left(\mathbb{1}-\sigma_{A}^{i} \otimes \sigma_{B}^{i}\right) / 4\right\}=-2$, which is not acceptable. The occurrence of a negative cross section comes from the non-positivity of $\mathbb{1}+\sigma_{A}^{i} \otimes \sigma_{B}^{i}$. This example shows that positivity has to be tested not only with factorised or separable states, but also with entangled ones. The latter impose non-classical conditions, stronger than the classical ones. More details are given in Sec. 5.3.

Similarly, a reduced CSM of the final particles $\mathcal{R}_{C D} \propto\left(\mathbb{1}+\sigma_{C}^{i} \otimes \sigma_{D}^{i}\right) / 4$ is classically, but not quantum-mechanically acceptable. In fact, quantum mechanics does not allow particles $C$ and $D$ to have their three spin components equal.

An analogous phenomenon occurs for correlations between initial and final spins. Suppose that the reduced CSM $\mathcal{R}_{A, C}$ defined by (2.74) takes the form $\mathcal{R}_{A, C} \propto \mathbb{1}-\sigma_{A}^{i} \otimes\left(\sigma_{C}^{i}\right)^{t}$, equivalent to $(i 0 \mid j 0)=-\delta_{i j}$. For independent $\boldsymbol{S}_{A}$ and $\boldsymbol{S}_{C}, d \sigma / d \Omega \propto 1-\boldsymbol{S}_{A} \cdot \boldsymbol{S}_{C} \geq 0$ or, using (2.75), $\left\langle\boldsymbol{S}_{C}\right\rangle=-\boldsymbol{S}_{A}$. This complete reversal of all spin components is acceptable classically, but not quantum-mechanically. Consider for instance the reaction

$$
\pi^{+}+{ }^{4} \mathrm{He} \rightarrow \pi^{0}+\mathrm{p}+{ }^{3} \mathrm{He},
$$

which, in the impulse approximation, can be decomposed into

$$
\text { (i) } \quad{ }^{4} \mathrm{He} \rightarrow \mathrm{n}+{ }^{3} \mathrm{He}, \quad \text { (ii) } \quad \pi^{+}+\mathrm{n} \rightarrow \pi^{0}+\mathrm{p} .
$$

We neglect off mass shell effects, final state interactions, and assume that $n+{ }^{3} \mathrm{He}$ is in the singlet ${ }^{1} \mathrm{~S}_{0}$ wave. Then $\mathrm{n}$ and ${ }^{3} \mathrm{He}$ have all spin components opposite. A complete reversal of the nucleon 


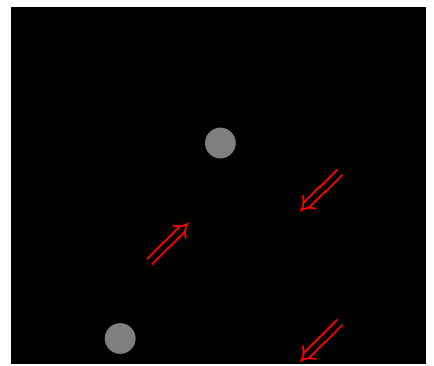

Figure 5: Mechanism for the reaction $\pi^{+}+$ ${ }^{4} \mathrm{He} \rightarrow \pi^{0}+\mathrm{p}+{ }^{3} \mathrm{He}$. The double arrows represent intermediate and final polarisations in the forbidden case $\boldsymbol{S}_{p}=-\boldsymbol{S}_{n}$.

spin in reaction (ii) would lead to fully equal ${ }^{3} \mathrm{He}$ and $\mathrm{p}$ spins in the final state. This, as shown before, is forbidden. The defect lies in the non-positiveness of $\mathbb{1}-\sigma_{A}^{i} \otimes\left(\sigma_{C}^{i}\right)^{t}$. Quantum mechanics does not allow full spin reversal.

The second counter-example is related to the first one by crossing. A CSM of the form $\mathbb{1}-$ $\boldsymbol{\sigma}_{A} . \boldsymbol{\sigma}_{C}^{t}$ gives a negative cross section when tested with the entangled " $t$-channel spin-singlet projector" $\left(\mathbb{1}+\boldsymbol{\sigma}_{A} \cdot \boldsymbol{\sigma}_{C}^{t}\right) / 4$. The lesson of these examples is that positivity has to be tested with classical and entangled states, i.e., which cannot be written as (see Eq. (2.57)), in the direct and crossed channels.

\subsection{Search for the positivity domain of a subset of observables}

\subsubsection{Simulation method}

Suppose a reaction described by a set of amplitudes $a, b, \ldots$, in terms of which the differential cross section is $I=|a|^{2}+|b|^{2}+\cdots$ and the spin observables $\left\langle\mathcal{O}_{i}\right\rangle$ (times $I$ ) are given by quadratic expressions such as $I\left\langle\mathcal{O}_{i}\right\rangle=|a|^{2}-|b|^{2}, \cdots, I\left\langle\mathcal{O}_{j}\right\rangle=2 \Re \mathrm{e}\left(a b^{*}\right)+\cdots$, etc. A simple method to detect inequalities among observables consists of generating random, fictitious amplitudes $a, b$, etc. ( $a$ can be chosen real and positive), computing the $\left\langle\mathcal{O}_{i}\right\rangle$, and plotting the results for one observable against another one. Once the contours revealed, it can be attempted to demonstrate rigorously the corresponding inequalities.

The method works quite well till about 6 amplitudes, without the need for sophisticated algorithms. This will be illustrated in the case of $\bar{p} p \rightarrow \bar{\Lambda} \Lambda$. If the number of amplitudes becomes larger, as in the case of the photoproduction of vector mesons, the plots are mostly populated in the centre, and the contour of the domain is less visible. In this case, one should force the random generator to explore the corners.

\subsubsection{Anticommutation and spherical constraints.}

Many constraints for reactions with spin one-half particles or photons are of the type

$$
\left\langle\mathcal{O}_{1}\right\rangle^{2}+\left\langle\mathcal{O}_{2}\right\rangle^{2}+\cdots \leq 1
$$

Such disk domains for pairs of observables or spherical domains for triples of observables are, in many cases, straightforward results of anticommutation of the observables. Let us recall that the observable $\langle\mathcal{O}\rangle=C_{\lambda \mu \nu \tau}$ can be considered as the expectation value of the partially transposed operator $\mathcal{O}^{\mathrm{pt}}=\sigma_{\lambda}(A) \otimes \sigma_{\mu}(B) \otimes \sigma_{\nu}^{t}(C) \otimes \sigma_{\tau}^{t}(D)$, according to (2.70). Since $\sigma_{\mu}^{2}=\mathbb{1}_{2}$ for each $\mu$, we have $\left(\mathcal{O}^{\mathrm{pt}}\right)^{2}=\mathcal{O}^{2}=\mathbb{1}$. Furthermore two such operators $\mathcal{O}^{\mathrm{pt}}$ and $\mathcal{O}^{\text {pt }}$ differing by some indices $\lambda, \mu, \nu$ or $\tau$ either commute or anticommute. We will forget below the superscript "pt", since partial transposition does not change the (anti-) commutation properties of $\sigma$-matrices.

For pairs of anticommuting observables, disk domains result from the following theorem [61]:

$$
\text { If } \mathcal{O}^{2}=\mathcal{O}^{\prime 2}=\mathbb{1} \text { and } \mathcal{O} \text { and } \mathcal{O}^{\prime} \text { are anticommuting, then }\langle\mathcal{O}\rangle^{2}+\left\langle\mathcal{O}^{\prime}\right\rangle^{2} \leq 1 .
$$


Proof: set $x=\sqrt{\langle\mathcal{O}\rangle^{2}+\left\langle\mathcal{O}^{\prime}\right\rangle^{2}},\langle\mathcal{O}\rangle=a x,\left\langle\mathcal{O}^{\prime}\right\rangle=b x$. Then $a^{2}+b^{2}=1$ and $\left\langle a \mathcal{O}+b \mathcal{O}^{\prime}\right\rangle=x$. From $\mathcal{O}^{2}=\mathcal{O}^{\prime 2}=\mathbb{1}$ and $\mathcal{O O}^{\prime}+\mathcal{O}^{\prime} \mathcal{O}=0$ one gets $\left(a \mathcal{O}+b \mathcal{O}^{\prime}\right)^{2}=\mathbb{1}$ which means that $a \mathcal{O}+b \mathcal{O}^{\prime}$ has eigenvalues \pm 1 . Its expectation value $x$ has to be within these eigenvalues, therefore $x^{2} \leq 1$. Generalisation: if $\mathcal{O}_{1}, \mathcal{O}_{2}, \ldots \mathcal{O}_{N}$ are mutually anticommuting and their squares are the unit matrix, then $\left\langle\mathcal{O}_{1}\right\rangle^{2}+\left\langle\mathcal{O}_{2}\right\rangle^{2}+\ldots+\left\langle\mathcal{O}_{N}\right\rangle^{2} \leq 1$. These results apply also to reactions involving photons and gravitons, which are two-components objects.

Note that disk or sphere can occur even if the observables commute, for instance if, due to some symmetry, $\mathcal{O}_{2}$ has the same expectation value as another operator $\mathcal{O}_{2}^{\prime}$ which anticommutes with $\mathcal{O}_{1}$ and $\mathcal{O}_{3}$. Examples of this situation are indicated by crossed circles in Table 4 of Sec. 3.3.3.

\subsubsection{Number of amplitudes and existence of constraints}

Suppose that, for instance, 3 observables $\mathcal{O}_{1}, \mathcal{O}_{2}$, and $\mathcal{O}_{3}$ of eigenvalues -1 and +1 are commuting and not related to anticommuting pairs by some symmetry, so that their joint positivity domain $\mathcal{D}_{3}$ may be larger than the unit ball. Can this domain be the whole cube? A partial negative answer is the following: If the reaction depends on $N$ independent amplitudes, $\mathcal{D}_{3}$ can reach at most $N$ corners of the cube. The proof is the following:

1. There is a one-to-one correspondence between matrix amplitudes $\mathcal{M}$ an state vectors $|\Psi\rangle$ of the $\overline{A B} C D$ channel, defined by

$$
\langle c, d|\mathcal{M}| a, b\rangle=\langle\Psi \mid a, b, c, d\rangle
$$

the observable $\langle\mathcal{O}\rangle$ reads

$$
\langle\mathcal{O}\rangle=\left\langle\Psi\left|\mathcal{O}^{\mathrm{pt}}\right| \Psi\right\rangle
$$

Here again we will forget the superscript "pt".

2. the number of independent amplitudes is the dimensionality of the Hilbert space of the $|\Psi\rangle$ 's.

3. Since $\mathcal{O}_{1}, \mathcal{O}_{2}$, and $\mathcal{O}_{3}$ commute, let us take a basis in which they are all diagonal. Consider a corner $\left(x_{1}, x_{2}, x_{3}\right)$ of the cube (each $x_{i}= \pm 1$ ). If $\mathcal{D}_{3}$ touches this corner there exists a $\mathcal{M}$ - matrix and the corresponding state $|\Psi\rangle$ for which $\left\langle\Psi\left|\left(\mathcal{O}_{1}, \mathcal{O}_{2}, \mathcal{O}_{3}\right)\right| \Psi\right\rangle=\left(x_{1}, x_{2}, x_{3}\right)$. This state is necessarily an eigenstate of $\left(\mathcal{O}_{1}, \mathcal{O}_{2}, \mathcal{O}_{3}\right)$, since $x_{1}, x_{2}$ and $x_{3}$ are extremal eigenvalues. It can be written as $\left|x_{1}, x_{2}, x_{3}, \nu\right\rangle$ where $\nu$ stands for possible additional quantum numbers. Two states $\left|x_{1}, x_{2}, x_{3}, \nu\right\rangle$ and $\left|x_{1}^{\prime}, x_{2}^{\prime}, x_{3}^{\prime}, \nu^{\prime}\right\rangle$ differing by at least one label are orthogonal.

4. if $N_{c}$ corners are occupied, there are at least $N_{c}$ mutually orthogonal, hence independent, $|\Psi\rangle$ 's. Thus $N_{c}$ cannot exceed the number of independent amplitudes

As an example, in the $\bar{p} p \rightarrow \bar{\Lambda} \Lambda$ reaction which depends on 6 amplitudes, only 6 corners of a cube can be reached for a triple of observables. See, e.g., Fig. 21 for the case of $\bar{p} p \rightarrow \bar{\Lambda} \Lambda$.

\subsubsection{Methods of the apparent contour and of the reciprocal polar transform}

The method based on anticommutation relations is efficient for finding circular or spherical domains. When it does not give any constraint, the domain may be the square or the cube, or smaller. To get the exact domain, a lengthy but straightforward method consists in determining the apparent contour of the positivity domain on the observable plane with the help of differential equations. An example is given in Sec.3.3.4.

A more elegant method is based on the polar reciprocity between intersection and projection [40], which can be derived from (2.23). Let us show how it applies to a reaction $1 / 2+1 / 2 \rightarrow$ $1 / 2+1 / 2$. Instead of a unit trace density matrix one works with the cross section matrix normalized to $\operatorname{Tr} \widehat{\mathcal{R}}=\operatorname{Tr}\left(\mathbb{1}_{N}\right)=N$ (here $N=24$ ). Following its decomposition (2.73) into usual observables, 
$\widehat{\mathcal{R}}$ is represented by a $N 2$-dimensional Euclidean vector $\boldsymbol{C}=\left(C_{0000}, C_{0001}, \ldots\right) \equiv\left(C_{0000}, \boldsymbol{C}_{\perp}\right)$ with $C_{0000}=1$ and positivity confines $C_{\perp}$ in a $N^{2}-1$ dimensional domain $\mathcal{D}$. The scalar product of two vectors is

$$
(1 / N) \operatorname{Tr}\left\{\widehat{\mathcal{R}} \widehat{\mathcal{R}}^{\prime}\right\}=\boldsymbol{C} \cdot \boldsymbol{C}^{\prime}=1+\boldsymbol{C}_{\perp} \cdot \boldsymbol{C}_{\perp}^{\prime}
$$

and by analogy with (2.21), we have

$$
C_{\perp} \cdot C_{\perp}^{\prime} \geq-1
$$

for $C_{\perp}$ and $C_{\perp}^{\prime} \in \mathcal{D}$. Let us compare the projected domain $\mathcal{D}_{3}\left\{\mathcal{O}_{1}, \mathcal{O}_{2}, \mathcal{O}_{3}\right\}$ of a triplet of observables and the intersection $\mathcal{I}_{3}$ of $\mathcal{D}$ with the plane $\left(\left\langle\mathcal{O}_{4}\right\rangle, \ldots\left\langle\mathcal{O}_{N^{2}-1}\right\rangle\right)=0$. In analogy with (2.23), (2.84) is also satisfied for $C_{\perp} \in \mathcal{D}_{3}$ and $C_{\perp}^{\prime} \in \mathcal{I}_{3}$, therefore the boundaries $\partial \mathcal{D}_{3}$ and $\partial \mathcal{I}_{3}$ are reciprocal under a polar transformation of power -1 . This is also the case when parity, charge conjugation, etc. have been taken into account.

This property simplifies the drawing of $\mathcal{D}_{3}\left\{\mathcal{O}_{1}, \mathcal{O}_{2}, \mathcal{O}_{3}\right\}$ : we first analyse the different pieces of $\partial \mathcal{I}_{3}$. This is an easy task since the unobserved variables are just dropped from the equalities and inequalities defining $\mathcal{D}_{\text {eff. }}$. Then we apply the polar transformation, edge by edge following (2.20). An example of application of this method will be given in Sec. 3.3.4.

\subsection{Quadratic identities between observables}

For exclusive reactions described by $n$ independent amplitudes, the matrix $\mathcal{R}$ is of rank one and each $p \times p$ subdeterminant with $p \geq 2$ vanishes. Then all the nonlinear positivity bounds are saturated. The vanishing of the $2 \times 2$ determinants (which lead to the vanishing of the higher order ones) gives $[n(n-1) / 2]^{2}$ real quadratic relations between the $n^{2}$ real parameters of the density matrix [44-46]. These relations are redundant and only $(n-1)^{2}$ of them can be independent, since the observables depend on $2 n-1$ independent real parameters. Consider, for instance, the quadratic relations containing $\rho_{11}$ :

$$
\rho_{11} \rho_{i k}-\rho_{i 1} \rho_{1 k}=0 .
$$

They represent $(n-1) 2$ independent real equations. If $\rho_{11} \neq 0$, one gets the factorised form of $\rho_{i k}$ :

$$
\rho_{i k}=\rho_{i 1}\left(\rho_{1 k} / \rho_{11}\right)=f(i) g(k) .
$$

This factorisation means that $\rho$ is of rank 1 and all the other $2 \times 2$ determinants vanish. Unfortunately, if $\rho_{11}=0$, Eq. (2.85) implies $\rho_{i 1}=\rho_{1 i}=0$ for all $i$ and the only constraint is that the rank is less than $n$. Other subsets of $(n-1)^{2}$ independent $2 \times 2$ relations can be chosen, but again auxiliary inequalities are necessary. For instance the vanishing of the $(n-1) 2$ subdeterminants formed by the intersection of two consecutive columns with two consecutive lines induces the vanishing of all the other determinants if $\rho_{22} \rho_{33} \ldots \rho_{n n} \neq 0$.

\subsection{Symmetry constraints on observables}

In this section we will present the constraints obtained from various symmetry requirements.

\subsubsection{Rotation invariance (conservation of angular momentum)}

Rotation invariance is implicitly taken into account in Eqs. (2.3-2.4), for instance, because the spin components are measured in a coordinate frame linked to the particle momenta and the coefficients only depend on the kinematical invariants.

Some reactions have cylindrical symmetry about some momentum $p$ : forward scattering, total cross section $(A+B \rightarrow X)$, two-particle decay, inclusive decay $(A \rightarrow B+X)$. In these cases the number of independent polarised observables is significantly reduced. As an example, let us treat the decay $\Lambda \rightarrow p+\pi^{-}$. In the $\Lambda$ rest frame, one may define the asymmetry parameters $(\lambda \mid \mu)$ with respect to a triad $\{\boldsymbol{l}, \boldsymbol{m}, \boldsymbol{n}\}$ where $\boldsymbol{l}$ is along the direction $\hat{\boldsymbol{p}}$ of the proton, $\boldsymbol{m}$ along $\hat{\boldsymbol{z}} \times \hat{\boldsymbol{p}}$, where 
$\hat{\boldsymbol{z}}$ is an arbitrarily chosen direction of space, and $\boldsymbol{n}=\boldsymbol{l} \times \boldsymbol{m}$. Due to conservation of $J . \hat{\boldsymbol{p}}$, there is only two non-vanishing helicity amplitudes, $\langle+|\mathcal{M}|+\rangle$ and $\langle-|\mathcal{M}|-\rangle$. Then the CSM, which is a $4 \times 4$ matrix is of the Table 1 type, has vanishing elements except in the $2 \times 2$ diagonal block acting on the $\{|++\rangle,|--\rangle\}$ subspace. This leads to $(l \mid l)=1,(0 \mid l)=(l \mid 0) \equiv \alpha$ in the notation of the Particle Data Group [62], $(m \mid m)=(n \mid n) \equiv \gamma$ and $-(m \mid n)=(n \mid m) \equiv \beta$, all other $(\lambda \mid \mu)$ being zero. Furthermore, it has rank one, whence the quadratic constraint

$$
\alpha^{2}+\beta^{2}+\gamma^{2}=1 .
$$

The analysing power $\alpha$ governs the angular distribution in the $\Lambda$ frame, $d N / d \Omega \propto 1+\alpha \hat{\boldsymbol{p}} . \boldsymbol{P}_{\Lambda}$, where $\hat{\boldsymbol{p}}=\boldsymbol{p} /|\boldsymbol{p}|$. It is non-zero due to parity non-conservation in weak interactions. The experimental value for $\Lambda \rightarrow p+\pi^{-}$is $\alpha=0.642 \pm 0.013$. The other independent parameter is

$$
\phi=\arg (\gamma+i \beta)=\phi\left(\boldsymbol{S}_{\Lambda}\right)-\phi\left(\boldsymbol{S}_{p}\right)=\arg \left(\frac{\mathcal{M}_{++}}{\mathcal{M}_{--}}\right) .
$$

The proton transversity is rotated from the $\Lambda$ one by $-\phi$. Details can be found in [62].

The same conclusions can be simply obtained using (2.66) and the rotation-invariant form,

$$
\left\langle\boldsymbol{s}_{p}|\mathcal{M}| \boldsymbol{s}_{\Lambda}\right\rangle=\left\langle\boldsymbol{s}_{p}\left|a_{S}+a_{P} \sigma \cdot \hat{\boldsymbol{p}}\right| \boldsymbol{s}_{\Lambda}\right\rangle,
$$

of the amplitude. $a_{S}$ and $a_{P}$ are S- and P-wave amplitudes and, according to Watson's theorem, their phases are the S- and P-wave phase shifts $\delta_{S}$ and $\delta_{P}$. A difference between these phase shifts leads to a non-vanishing $\phi$, owing to $\mathcal{M}_{++}=a_{S}+a_{P}, \mathcal{M}_{--}=a_{S}-a_{P}$.

Another example is provided by quark distributions, i.e., the reaction $N \rightarrow q+X$ (for the definitions, see Sec. 4.3. After integration over the intrinsic transverse momentum, they are invariant by rotation about the nucleon momentum axis $l$. The cross section matrix is again of the type of Table 1, but $\left\langle\lambda_{N}^{\prime}, \lambda_{q}^{\prime}|\mathcal{R}| \lambda_{N}, \lambda_{q}\right\rangle$ is zero unless $\lambda_{N}^{\prime}-\lambda_{q}^{\prime}=\lambda_{N}-\lambda_{q}\left(=\lambda_{X}\right.$, due to conservation of $\boldsymbol{J}_{l}$ ). This leaves only the diagonal elements and the upper-right and lower-left corners. Furthermore parity conservation imposes $(0 \mid l)=(l \mid 0)=(m \mid n)=(n \mid m)=0$. One is left with $(0 \mid 0)=1$, $(l \mid l)=\Delta q(x) / q(x)$ and $(m \mid m)=(n \mid n)=\delta q(x) / q(x)$, where $q(x)$ is the unpolarised quark distribution, $\Delta q(x)$ and $\delta q(x)$ are the helicity and transversity distributions. Part of the parent hadron helicity is taken by other partons and the orbital angular momenta, therefore $(l \mid l)<1$, unlike in the $\Lambda \rightarrow p \pi$ case. Positivity of $\mathcal{R}$ is insured by $\Delta q(x) / q(x) \leq 1, \delta q(x) / q(x) \leq 1$ and the Soffer inequality, discussed in Sec. 4.3.1,

$$
2|\delta q(x)| \leq q(x)+\Delta q(x) .
$$

Similar conclusions hold for the quark fragmentation into baryon $q \rightarrow B+X$.

\subsubsection{Parity conservation (in strong and electromagnetic interactions)}

Case of planar reactions The space inversion operator (parity) $P$ transforms a reaction into another one with the same spin vectors but opposite particle momenta. In $2 \rightarrow 2$ scattering or in 3-body decay, one can define a reaction plane (scattering plane or decay plane). One can also define such a plane in 1-particle inclusive inelastic scattering or 2-particle inclusive decay. The set of observed momenta is invariant under the mirror reflection with respect to the reaction plane, described by the operator

$$
\Pi=P \exp (-i \pi \boldsymbol{J} \cdot \boldsymbol{n}) .
$$

$\boldsymbol{J}$ is the total angular momentum. $\Pi$ is unaffected by a translation or a boost parallel to the reaction plane. The action of $\Pi$ on one-particle helicity states is given by

$$
\Pi|\theta, \phi, \lambda\rangle=\eta(-1)^{s-\lambda}|\theta,-\phi,-\lambda\rangle .
$$


Inequalities on spin observables

Here $\eta$ is the intrinsic parity of the particle, e.g., -1 for the photon and the pion ${ }^{6}$.

If parity is conserved, it is advantageous to use the transversity basis or, for spin-1 particles, the states linearly polarised parallel or normal to the scattering plane defined in Secs. 2.3. For a particle in the scattering plane $(\phi=0$ or $\pm \pi$ ), these states are eigenstates of $\Pi$ :

$$
\begin{aligned}
\operatorname{spin} 1 / 2: & \Pi| \pm \boldsymbol{n}\rangle & =\mp i \eta| \pm \boldsymbol{n}\rangle, \\
\operatorname{spin} 1: & \Pi\{|\boldsymbol{n}\rangle,|\boldsymbol{\pi}\rangle,|\boldsymbol{l}\rangle\} & =\{\eta|\boldsymbol{n}\rangle,-\eta|\boldsymbol{\pi}\rangle,-\eta|\boldsymbol{l}\rangle\} .
\end{aligned}
$$

By parity conservation, the amplitude $\mathcal{M}$ of $A+B \rightarrow C+D$ fulfils:

$$
\mathcal{M}=\left(\Pi_{C} \otimes \Pi_{D}\right)^{-1} \mathcal{M}\left(\Pi_{A} \otimes \Pi_{B}\right) .
$$

Applying this equation to both $\mathcal{M}$ and $\mathcal{M}^{\dagger}$ in Eq. (2.68) for the cross section matrix leads to

$$
\widetilde{\mathcal{R}}=\left(\Pi_{A} \otimes \Pi_{B} \otimes \Pi_{C} \otimes \Pi_{D}\right)^{-1} \widetilde{\mathcal{R}} \Pi_{A} \otimes \Pi_{B} \otimes \Pi_{C} \otimes \Pi_{D} .
$$

For the observables one has, using (2.66) or (2.70),

$$
\left\langle\mathcal{O}_{i} \mathcal{O}_{f}\right\rangle=\left\langle\mathcal{O}_{i}^{\Pi} \mathcal{O}_{f}^{\Pi}\right\rangle
$$

where $\mathcal{O}^{\Pi} \equiv \Pi \mathcal{O} \Pi^{-1}$. The transformation $\mathcal{O} \rightarrow \mathcal{O}^{\Pi}$ is given below for the operators defined in Sec. 2.3.1-2.3.2:

$$
\begin{aligned}
& \text { Spin 1/2: } \quad\left(\sigma_{l}, \sigma_{m}, \sigma_{n}\right) \rightarrow\left(-\sigma_{l},-\sigma_{m}, \sigma_{n}\right) . \\
& \text { Photon, gluon : } \quad\left(\sigma_{\odot}, \sigma_{\oslash}, \sigma_{\ominus}\right) \rightarrow\left(-\sigma_{\odot},-\sigma_{\oslash}, \sigma_{\ominus}\right) \text {. }
\end{aligned}
$$

Similar relations for the massive spin-1 particles can be derived from (2.93) and are given in Sec. 3.5. Usual one-particle observables $O_{\lambda}$ are either even or odd under $\Pi$. This is also true for the multi-spin observable $O_{\lambda \mu \nu \ldots}=O_{\lambda}(A) \otimes O_{\mu}(B) \otimes O_{\nu}(C) \ldots$, since parity is multiplicative. From (2.96) one obtains the following "classical" rule:

\section{If parity is conserved, all П-odd observables vanish.}

This rule roughly reduces by a factor 2 the number of observables. It does not depend on the intrinsic parity of the particles. It just expresses a classical requirement of reflection symmetry at the level of polarised cross sections. It is fulfilled, for instance, by formula (2.6). A well-known counter-example is $\beta$-decay, e.g., $\mathrm{n} \rightarrow \mathrm{p}+\mathrm{e}^{-}+\bar{\nu}$, where the outgoing electron has longitudinal polarisation.

Less trivial, "non-classical", constraints are obtained by applying (2.94) only to $\mathcal{M}$ or only to $\mathcal{M}^{\dagger}$ in $(2.68)$ or $(2.66-2.67)$ :

$$
\begin{aligned}
\widetilde{\mathcal{R}} & =\Pi_{C}^{-1} \otimes \Pi_{D}^{-1} \widetilde{\mathcal{R}} \Pi_{A} \otimes \Pi_{B}, \\
\left\langle\mathcal{O}_{i} \mathcal{O}_{f}\right\rangle & =\left\langle\left(\Pi_{i} \mathcal{O}_{i}\right)\left(\mathcal{O}_{f} \Pi_{f}^{-1}\right)\right\rangle .
\end{aligned}
$$

For a pseudoscalar meson, $\Pi=-1$. For the photon or gluon, $\Pi=\sigma_{\ominus}$. For spin-1/2, $\Pi=-i \eta \sigma_{n}$, so

$$
\Pi\left(\sigma_{0}, \sigma_{l}, \sigma_{m}, \sigma_{n}\right)=\eta\left(-i \sigma_{n}, \sigma_{m},-\sigma_{l},-i \sigma_{0}\right) .
$$

The non-classical parity constraint in the case of spin-1/2 particles are known as the Bohr identities [63].

Equation (2.99) or (2.100) implies the classical parity rule (2.95) or (2.96). In addition, it yields linear identities between the $\Pi$-even observables, which depend on the intrinsic parities and cannot

\footnotetext{
${ }^{6} \eta$ is a complex number of modulus one. The $\eta$ 's of non self-conjugate particles may be multiplied by collective phase factors which, in any reaction, do not change the product of the initial $\eta$ 's, divided by the final ones. It is natural to choose the same $\eta$ within a $S U(3)$ multiplet. The intrinsic parities $\eta(a)$ and $\eta(\bar{a})$ of a fermion and its antiparticle are such that $\eta(a) \eta(\bar{a})=-1$
} 
be obtained from purely classical arguments. These identities reduce the number of independent correlation parameters $(\lambda \mu \mid \nu \ldots)$ roughly by another factor 2 . For instance, in $\pi^{0}$ decay, the classical rule (2.96) says that the linear polarisations of the two gamma's are either parallel or orthogonal (not, e.g. at $\pi / 4$ ). The non-classical rule (2.100) selects the orthogonal solution.

The subdivision in constraints of the (2.96) and (2.100) types, both for parity and time-reversal, was made in Appendix 3.D. of [1]. In this section we point out the "classical" versus "non-classical" or "quantum" characters of these two types [60]. As a matter of fact, inclusive reactions have only "classical constraints", since the intrinsic parity of the undetected particles takes both signatures. The same is true for partially polarised exclusive reactions. The disappearance of non-classical constraints as information is lost has relationship with decoherence of incompletely controlled quantum systems. This classical vs. quantum distinction will also apply below to charge conjugation and particle indiscernability.

Case of non-planar reactions $\quad$ In $2 \rightarrow n$ exclusive scattering with $n \geq 3$, for instance $\pi+\mathrm{N} \rightarrow$ $\pi+\pi+\mathrm{N}$, or in $1 \rightarrow n$ decay with $n \geq 4$ the momenta are not coplanar and cannot be invariant under a mirror reflection like (2.91). This is also the case of two-particle inclusive scattering, etc. Using the ordinary operator $P$, parity conservation for a $2 \rightarrow 3$ reaction reads

$$
\mathcal{M}\left(\boldsymbol{p}_{A}, \boldsymbol{p}_{B}, \boldsymbol{p}_{C}, \boldsymbol{p}_{D}, \boldsymbol{p}_{E}\right)=\left(P_{C} \otimes P_{D} \otimes P_{E}\right)^{-1} \mathcal{M}\left(-\boldsymbol{p}_{A},-\boldsymbol{p}_{B},-\boldsymbol{p}_{C},-\boldsymbol{p}_{D}-\boldsymbol{p}_{E}\right)\left(P_{A} \otimes P_{B}\right) .
$$

Following the same steps as in Eqs.(2.94-2.96) one obtains the classical constraint

$$
\left\langle\mathcal{O}_{i} \mathcal{O}_{f}\right\rangle_{\boldsymbol{p}_{A}, \boldsymbol{p}_{B}, \boldsymbol{p}_{C}, \boldsymbol{p}_{D}, \boldsymbol{p}_{E}}=\left\langle\mathcal{O}_{i}^{P} \mathcal{O}_{f}^{P}\right\rangle_{-\boldsymbol{p}_{A},-\boldsymbol{p}_{B},-\boldsymbol{p}_{C},-\boldsymbol{p}_{D},-\boldsymbol{p}_{E}} .
$$

where $\mathcal{O}^{P} \equiv P \mathcal{O} P^{-1}$. In particular $\sigma^{P}=\sigma$. Equivalently one can chose an arbitrary plane containing the $z$-axis and use the reflection operator (2.91) about this plane. Then,

$$
\left\langle\mathcal{O}_{i} \mathcal{O}_{f}\right\rangle_{\phi_{A}, \phi_{B}, \phi_{C}, \phi_{D}, \phi_{E}}=\left\langle\mathcal{O}_{i}^{\Pi} \mathcal{O}_{f}^{\Pi}\right\rangle_{-\phi_{A},-\phi_{B},-\phi_{C},-\phi_{D},-\phi_{E}} .
$$

This is the result obtained in [64] with the help of helicity amplitudes. In practice $\phi_{A}=\phi_{B}=0$ and one links the $\phi=0$ plane to a final momentum, for instance to $\left(\boldsymbol{p}_{C}+\boldsymbol{p}_{D}\right)_{\perp}=-\left(\boldsymbol{p}_{E}\right)_{\perp}$ if $C$ and $D$ can make a resonance. Then $\phi_{C}$ and $\phi_{D}$ can be replaced by only one azimuthal angle or by $\boldsymbol{p}_{C} . \boldsymbol{n}$, which is also $\Pi$-odd.

Unfortunately, one cannot derive non-classical parity constraints like (2.100). Indeed, applying (2.102) only to $\mathcal{M}$ or only to $\mathcal{M}^{\dagger}$ in the analogue of (2.66), amounts to consider an interference between two amplitudes taken at different momenta, a quantity which is not accessible experimentally.

\subsubsection{Permutations of $A \leftrightarrow B$ and/or $C \leftrightarrow D$}

Preliminary: some transformations of helicity states. Let us generalise (2.53) by

$$
\left|\boldsymbol{k} ; A_{1}, \lambda_{1} ; A_{2}, \lambda_{2}\right\rangle=a^{\dagger}\left(\boldsymbol{k} ; A_{1}, \lambda_{1}\right) a^{\dagger}\left(-\boldsymbol{k} ; A_{2}, \lambda_{2}\right)|\varnothing\rangle,
$$

which takes Bose or Fermi statistics into account for identical particles, $A_{1}=A_{2}$. The (anti-) commutation relations between creation operators give

$$
\left|\boldsymbol{k} ; A_{1}, \lambda_{1} ; A_{2}, \lambda_{2}\right\rangle=\varepsilon_{12}\left|-\boldsymbol{k} ; A_{2}, \lambda_{2} ; A_{1}, \lambda_{1}\right\rangle,
$$

with $\varepsilon_{12}=+1$ for a two-boson or boson-fermion pair, $\varepsilon_{12}=-1$ for a two-fermion pair.

If $\boldsymbol{k}$ is in the $(x, z)$ half-plane with $\phi(\boldsymbol{k})=0$, a rotation of angle $+\pi$ about $\hat{\boldsymbol{y}}$ yields, according to the convention (2.51),

$$
\exp \left(-i \pi J_{y}\right)\left|\boldsymbol{k} ; A_{1}, \lambda_{1} ; A_{2}, \lambda_{2}\right\rangle=\varepsilon_{1}\left|-\boldsymbol{k} ; A_{1}, \lambda_{1} ; A_{2}, \lambda_{2}\right\rangle,
$$


where $\varepsilon_{i} \equiv(-1)^{2 s_{i}}=+1$ for a boson, -1 for a fermion.

A rotation of a single particle about $\hat{z}$ gives

$$
\exp \left(-i u J_{z}\right)|\boldsymbol{p}(\theta, \phi), \alpha\rangle=\exp (-i \alpha u)\left|\boldsymbol{p}^{\prime}(\theta, \phi+u), \alpha\right\rangle .
$$

Applying it to the initial particle pair and restoring $\phi_{B}=\pi$, one obtains as expected

$$
\exp \left(-i \pi J_{z}\right)\left|k \hat{\boldsymbol{z}} ; A, \lambda_{A} ; B, \lambda_{B}\right\rangle=e^{i \pi\left(\lambda_{B}-\lambda_{A}\right)}\left|k \hat{\boldsymbol{z}} ; A, \lambda_{A} ; B, \lambda_{B}\right\rangle .
$$

These relations will be used together with rotational invariance,

$$
\mathcal{M}=\exp (i \boldsymbol{J} . \boldsymbol{r}) \mathcal{M} \exp (-i \boldsymbol{J} . \boldsymbol{r}) .
$$

Application to $A+B \rightarrow C+D$. From the preceding relations one can derive

$$
\begin{aligned}
& \left\langle\gamma, \delta\left|\mathcal{M}^{A B \rightarrow C D}(\theta)\right| \alpha, \beta\right\rangle=\varepsilon_{A} \varepsilon_{A B} \varepsilon_{C} \varepsilon_{C D}\left\langle\delta, \gamma\left|\mathcal{M}^{B A \rightarrow D C}(\theta)\right| \beta, \alpha\right\rangle, \\
& \left\langle\gamma, \delta\left|\mathcal{M}^{A B \rightarrow C D}(\theta)\right| \alpha, \beta\right\rangle=\varepsilon_{C D}(-1)^{\alpha-\beta-\gamma-\delta}\left\langle\delta, \gamma\left|\mathcal{M}^{A B \rightarrow D C}(\pi-\theta)\right| \alpha, \beta\right\rangle, \\
& \left\langle\gamma, \delta\left|\mathcal{M}^{A B \rightarrow C D}(\theta)\right| \alpha, \beta\right\rangle=\varepsilon_{A B}(-1)^{\alpha+\beta+\gamma-\delta}\left\langle\gamma, \delta\left|\mathcal{M}^{B A \rightarrow C D}(\pi-\theta)\right| \beta, \alpha\right\rangle .
\end{aligned}
$$

In Eq. (2.111), the product of $\varepsilon$ factors is +1 for the $\mathrm{bb} \rightarrow \mathrm{bb}$, ff $\rightarrow \mathrm{ff}, \mathrm{bb} \rightarrow \mathrm{ff}$, ff $\rightarrow \mathrm{bb}$, bf $\rightarrow \mathrm{bf}$, and $\mathrm{fb} \rightarrow \mathrm{fb}$ reactions ( $\mathrm{b}=$ boson, $\mathrm{f}=$ fermion), -1 for the $\mathrm{bf} \rightarrow \mathrm{fb}$ and $\mathrm{fb} \rightarrow$ bf reactions. It is obtained from (2.110) with $\boldsymbol{r}=\pi \hat{\boldsymbol{y}}$ and (2.106)-(2.107) applied to the $A+B$ and $C+D$ pairs. Equation (2.112) is obtained with $\boldsymbol{r}=\pi \hat{z},(2.109)$ and applying (2.108) and (2.106) to $C$ and $D$. Equation (2.113) results from (2.111) and (2.112).

\subsubsection{Identical particles}

For a pair of particles of equal spins, one defines the helicity exchange operator by

$$
P_{12}^{\text {hel. }}\left|\boldsymbol{k}, A_{1}, \lambda_{1} ; A_{2}, \lambda_{2}\right\rangle \equiv\left|\boldsymbol{k}, A_{1}, \lambda_{2} ; A_{2}, \lambda_{1}\right\rangle .
$$

Equation (2.111) applies to $A+A \rightarrow B+B$ with a + sign. It can be rewritten as

$$
P_{34}^{\text {hel. }} \mathcal{M} P_{12}^{\text {hel. }}=\mathcal{M} \text {. }
$$

For the cross section matrix, one obtains the "classical" and "non-classical" relations

$$
\begin{aligned}
& \mathcal{R}=\left(P_{12}^{\text {hel. }} \otimes P_{34}^{\text {hel. }}\right) \mathcal{R}\left(P_{12}^{\text {hel. }} \otimes P_{34}^{\text {hel. }}\right), \\
& \mathcal{R}=\mathcal{R}\left(P_{12}^{\text {hel. }} \otimes P_{34}^{\text {hel. }}\right) .
\end{aligned}
$$

For spins $1 / 2$, these relations give respectively

$$
\begin{aligned}
& (\lambda \mu \mid \nu \tau)=(\mu \lambda \mid \tau \nu), \\
& (\lambda \mu \mid \nu \tau)=\Gamma_{\lambda \lambda^{\prime} \mu \mu^{\prime}}\left(\lambda^{\prime} \mu^{\prime} \mid \nu^{\prime} \tau^{\prime}\right) \Gamma_{\nu^{\prime} \nu \tau^{\prime} \tau},
\end{aligned}
$$

where repeated indices are summed over and $\Gamma_{\alpha \beta \gamma \delta}=(1 / 4) \operatorname{Tr}\left\{\sigma_{\alpha} \sigma_{\beta} \sigma_{\gamma} \sigma_{\delta}\right\}$. Each of the 256 nonclassical relations (2.119) has 16 terms on the right-hand side. These relations are not independent and, by taking appropriate combinations, one obtains the shorter ones,

$$
\begin{array}{rlrl}
(0 i \mid p q)-(0 i \mid q p) & =(j k \mid r 0)-(j k \mid 0 r) & & \left(\varepsilon_{i j k}=\varepsilon_{p q r}=+1\right), \\
(i j \mid p q)-(i j \mid q p) & =(0 k \mid 0 r)-(0 k \mid r 0) & & (\text { idem }), \\
(x x \mid \nu \tau)+(y y \mid \nu \tau)+(z z \mid \nu \tau) & =(00 \mid \nu \tau) & & (\nu \neq \tau), \\
(00 \mid x x)+(00 \mid y y)+(00 \mid z z) & =(x x \mid 00)+(y y \mid 00)+(z z \mid 00), & \\
(00-x x \mid 00-x x) & =(y y+z z \mid y y+z z), & \\
2[(x x \mid y y)-(y y \mid x x)] & =(x x-y y \mid 00-z z)-(00-z z \mid x x-y y) .
\end{array}
$$


In the first two equations, $i j k$ and $p q r$ are even permutations of $x y z$ (here $\{x, y, z\} \equiv\{m, n, l\}$ ). A symmetric partner of the third equation is obtained for the $(\lambda \mu \mid \nu \nu)$ 's with $\lambda \neq \mu$. The last two equations plus their permutations of $\{x, y, z\}$ give $3+2$ independent relations. The notation $(x x-y y \mid 00-z z)$ stands for $(x x \mid 00)-(x x \mid z z)-(y y \mid 00)+(y y \mid z z)$. In total one has 36 independent relations.

Scattering at $\mathbf{9 0}^{\circ}$. Let us consider the case $A+A \rightarrow C+D$, where at least the initial particles are identical, for instance $\gamma \gamma \rightarrow \pi^{-} \rho^{+}$. At $\theta=90^{\circ}$ Eq. (2.113) gives

$$
\left\langle\gamma, \delta\left|\mathcal{M}^{A A \rightarrow C D}\left(90^{\circ}\right)\right| \alpha, \beta\right\rangle=(-1)^{\alpha-\beta+\gamma-\delta}\left\langle\gamma, \delta\left|\mathcal{M}^{A A \rightarrow C D}\left(90^{\circ}\right)\right| \beta, \alpha\right\rangle .
$$

For spin one-half fermions, it can be written as

$$
\mathcal{M}^{A A \rightarrow C D}\left(90^{\circ}\right)=\sigma_{l} \otimes \sigma_{l} \mathcal{M}^{A A \rightarrow C D}\left(90^{\circ}\right) P_{12}^{\text {hel }} \sigma_{l} \otimes \sigma_{l} .
$$

Applying this identity to both $\mathcal{M}$ and $\mathcal{M}^{\dagger}$ in (2.68) yields the classical symmetry relations

$$
(\lambda, \mu \mid \nu, \tau)=(\breve{\mu}, \breve{\lambda} \mid \breve{\nu}, \breve{\tau}) \quad(\theta=\pi / 2) .
$$

where $(\breve{\mu}, \breve{\lambda} \mid \breve{\nu}, \breve{\tau})=\left\langle\sigma_{\lambda} \sigma_{\nu} \check{\sigma}_{\mu} \breve{\sigma}_{\tau}\right\rangle$ and $\breve{\sigma}_{\alpha}=\sigma_{l} \sigma_{\alpha} \sigma_{l}$, more explicitly $\left\{\breve{\sigma}_{0}, \breve{\sigma}_{l}, \breve{\sigma}_{m}, \breve{\sigma}_{n}\right\}=\left\{\sigma_{0}, \sigma_{l}\right.$, $\left.-\sigma_{m},-\sigma_{n}\right\}$. Equation (2.123) predicts the vanishing of 32 Cartesian parameters like $(00 \mid 0 m)$, $(n n \mid m l)$ and yields 96 equivalences such as $(l 0 \mid l n)=-(0 l \mid l n),(l 0 \mid m n)=+(0 l \mid m n)$. These relations can be understood classically by the fact that the plane normal to the collision axis is a symmetry plane of the reaction.

Applying (2.122) only to $\mathcal{M}$ in (2.68) yields the non-classical relations

$$
(\lambda \mu \mid \nu \tau)=2^{-4}\left(\lambda^{\prime} \mu^{\prime} \mid \nu^{\prime} \tau^{\prime}\right) \operatorname{Tr}\left(\sigma_{\lambda} \sigma_{\lambda^{\prime}} \breve{\sigma}_{\mu} \breve{\sigma}_{\mu^{\prime}}\right) \operatorname{Tr}\left(\sigma_{l} \sigma_{\nu^{\prime}} \sigma_{\nu}\right) \operatorname{Tr}\left(\sigma_{l} \sigma_{\tau^{\prime}} \sigma_{\tau}\right) \quad(\theta=\pi / 2) .
$$

In particular,

$$
\begin{aligned}
2(00 \mid l l) & =+(00 \mid 00)+(l l \mid 00)-(m m \mid 00)-(n n \mid 00) \\
2(00 \mid m m) & =-(00 \mid n n)-(l l \mid n n)+(m m \mid n n)+(n n \mid n n) .
\end{aligned}
$$

If parity is conserved and the initial and final products of initial intrinsic parities are equal, one may transform (2.125-2.126) using the Bohr identity (2.101) to derive

$$
C_{l l}+C_{m m}+C_{n n}=1 .
$$

In a $A+A \rightarrow A+A$ reaction, $T$-invariance only gives $(00 \mid l l)=(l l \mid 00)$, therefore Eq. (2.125) reads

$$
A_{l l}+A_{m m}+A_{n n}=1,
$$

derived in [65] as $A_{N N}-A_{L L}-A_{S S}=1$, in the Argonne notation. For instance, in $\mathrm{e}^{-} \mathrm{e}^{-}$scattering, or the scattering of identical colourless quarks [66], one has, at $90^{\circ},\left(A_{n n}, A_{m m}, A_{l l}\right)=$ $(1 / 9,1 / 9,7 / 9)$. The quark interchange model [67] predicts $A_{n n}=A_{m m}=A_{l l}=1 / 3$ in highenergy pp scattering at $90^{\circ}$. When summation is made over the initial and final colours, one gets $\left(A_{n n}, A_{m m}, A_{l l}\right)=(-1 / 11,-1 / 11,5 / 11)$ in the scattering of identical quarks at $90^{\circ}$, and the relation (2.128) is no longer valid.

\subsubsection{Charge and $C P$ conjugations in $A+\bar{A} \rightarrow B+\bar{B}$}

Equation (2.111) also applies to $A+\bar{A} \rightarrow B+\bar{B}$ with a + sign. If $C$ is conserved, replacing each particle by its antiparticle does not change the amplitude, so that one again obtains Eqs. (2.1152.117) and, for one-half spins, Eqs. (2.118-2.120). 
$C P$ symmetry can be treated in a similar manner. Applying successively $P$ in the form (2.94), $C$ and (2.111) gives

$$
\mathcal{M}=\left(\Pi_{C} \otimes \Pi_{D}\right)^{-1} P_{C D}^{\text {hel. }} \mathcal{M} P_{A B}^{\text {hel. }}\left(\Pi_{A} \otimes \Pi_{B}\right) .
$$

For a fermion-antifermion pair, $\Pi_{1} \Pi_{2}=\sigma(1)_{y} \otimes \sigma(2)_{y}$. The classical $C P$ constraints may be obtained applying successively the transformations (2.97) and (2.118). The non-classical ones are of the form (2.119) with $\Gamma$ replaced by $\Gamma_{\lambda \lambda^{\prime} \mu \mu^{\prime}}^{C P}=(1 / 4) \operatorname{Tr}\left\{\sigma_{\lambda} \sigma_{\lambda^{\prime}} \sigma_{y} \sigma_{\mu} \sigma_{\mu^{\prime}} \sigma_{y}\right\}$. Alternatively, one may keep the formulas (2.119-2.120) but transforming the $(\lambda \mu \mid \nu \tau)$ 's according to

$$
(\lambda \mu \mid \nu \tau) \rightarrow(\lambda \hat{\mu} \mid \nu \hat{\tau})=\left\langle\sigma_{\lambda} \hat{\sigma}_{\mu} \sigma_{\nu} \hat{\sigma}_{\tau}\right\rangle,
$$

with $\hat{\sigma}_{\alpha} \equiv \sigma_{y} \sigma_{\alpha} \sigma_{y}$. For instance, the fourth Eq. (2.120) becomes $-(00 \mid x x)+(00 \mid y y)-(00 \mid z z)=$ $-(x x \mid 00)+(y y \mid 00)-(z z \mid 00)$ (here again $\{x, y, z\} \equiv\{m, n, l\})$. These constraints hold whether or not $P$ and $C$ are separately conserved.

\subsubsection{Time reversal invariance in $A+B \rightarrow A+B$}

We consider the two time-reversed elastic reactions

$$
\begin{aligned}
A\left(\boldsymbol{p}_{i}, \alpha_{i}\right)+B\left(\boldsymbol{k}_{i}, \beta_{i}\right) & \rightarrow A\left(\boldsymbol{p}_{f}, \alpha_{f}\right)+B\left(\boldsymbol{k}_{f}, \beta_{f}\right), \\
A\left(-\boldsymbol{p}_{f}, \alpha_{f}\right)+B\left(-\boldsymbol{k}_{f}, \beta_{f}\right) & \rightarrow A\left(-\boldsymbol{p}_{i}, \alpha_{i}\right)+B\left(-\boldsymbol{k}_{i}, \beta_{i}\right) .
\end{aligned}
$$

Here $\alpha$ and $\beta$ are helicities. At the level of amplitudes, time reversal invariance reads

$$
\langle f|\mathcal{M}| i\rangle=\left\langle i\left|T^{\dagger} \mathcal{M} T\right| f\right\rangle .
$$

A one-particle helicity state, for instance $|\boldsymbol{p}, \alpha\rangle$, transforms as

$$
T|\boldsymbol{p}, \alpha\rangle=\xi_{A} \exp \left(-2 i \alpha \phi_{\boldsymbol{p}}\right)|-\boldsymbol{p}, \alpha\rangle,
$$

where $\phi_{\boldsymbol{p}}$ is the azimuth of $\boldsymbol{p}$ and $\xi_{A}$ is a phase factor intrinsic to particle $A$.

The centre-of-mass momenta of reactions (2.131) and (2.132) are related by a symmetry about the momentum transfer $\boldsymbol{Q}=\boldsymbol{p}_{f}-\boldsymbol{p}_{i}$. We take the $z$-axis along $\boldsymbol{Q}$ and use rotation invariance to replace (2.133) by

$$
\langle f|\mathcal{M}| i\rangle=\left\langle i\left|T^{\dagger} \exp \left(i \pi J_{z}\right) \mathcal{M} \exp \left(-i \pi J_{z}\right) T\right| f\right\rangle .
$$

This equation relates two amplitudes of different helicities but identical momenta. It is valid in the centre-of-mass frame and any frame where $Q^{0}=0$, e.g., the Breit frames of $A$ and $B$. Using

$$
\exp \left(-i u J_{z}\right)|\boldsymbol{p}(\theta, \phi), \alpha\rangle=\exp (-i \alpha u)\left|\boldsymbol{p}^{\prime}(\theta, \phi+u), \alpha\right\rangle,
$$

equation (2.135) takes the explicit form

$$
\left\langle\alpha_{f}, \beta_{f}|\mathcal{M}| \alpha_{i}, \beta_{i}\right\rangle=(-1)^{\alpha_{i}-\alpha_{f}-\beta_{i}+\beta_{f}}\left\langle\alpha_{i}, \beta_{i}|\mathcal{M}| \alpha_{f}, \beta_{f}\right\rangle,
$$

omitting the momentum arguments. Applying this equation to both factors of (2.68), we obtain the "classical" time-reversal relation

$$
\left\langle\alpha_{i}^{\prime} \beta_{i}^{\prime} \alpha_{f}^{\prime} \beta_{f}^{\prime}|\mathcal{R}| \alpha_{i} \beta_{i} \alpha_{f} \beta_{f}\right\rangle=(-1)^{\alpha_{i}-\alpha_{i}^{\prime}+\alpha_{f}-\alpha_{f}^{\prime}+\beta_{i}-\beta_{i}^{\prime}+\beta_{f}-\beta_{f}^{\prime}}\left\langle\alpha_{f}^{\prime} \beta_{f}^{\prime} \alpha_{i}^{\prime} \beta_{i}^{\prime}|\mathcal{R}| \alpha_{f} \beta_{f} \alpha_{i} \beta_{i}\right\rangle .
$$

For observables $\mathcal{P}$ and $\mathcal{Q}$ dealing with the initial and final states separately, we have

$$
\langle\mathcal{P}(i) \mathcal{Q}(f)\rangle=\left\langle\mathcal{Q}^{R T}(i) \mathcal{P}^{R T}(f)\right\rangle,
$$

where $\mathcal{O}^{R T} \equiv \exp \left(-i \pi J_{z}\right) T \mathcal{O}^{t} T^{\dagger} \exp \left(i \pi J_{z}\right)$. For one-particle observables,

any spin: $\quad\left\langle\alpha\left|\mathcal{O}^{R T}\right| \alpha^{\prime}\right\rangle=(-1)^{\alpha-\alpha^{\prime}}\left\langle\alpha^{\prime}|\mathcal{O}| \alpha\right\rangle$,

spin 1/2: $\quad\left(\sigma_{x}, \sigma_{y}, \sigma_{z}\right)^{R T}=\left(-\sigma_{x}, \sigma_{y}, \sigma_{z}\right)$,

$$
\text { photon: } \quad\left(\sigma_{\ominus}, \sigma_{\oslash}, \sigma_{\odot}\right)^{R T}=\left(\sigma_{\ominus},-\sigma_{\oslash}, \sigma_{\odot}\right) \text {. }
$$


Applying (2.137) only to the second factor of (2.68) gives the "non-classical" relation

$$
\left\langle\alpha_{i}^{\prime} \beta_{i}^{\prime} \alpha_{f}^{\prime} \beta_{f}^{\prime}|\mathcal{R}| \alpha_{i} \beta_{i} \alpha_{f} \beta_{f}\right\rangle=(-1)^{\alpha_{i}-\alpha_{f}+\beta_{i}-\beta_{f}}\left\langle\alpha_{i}^{\prime} \beta_{i}^{\prime} \alpha_{f}^{\prime} \beta_{f}^{\prime}|\mathcal{R}| \alpha_{f} \beta_{f} \alpha_{i} \beta_{i}\right\rangle,
$$

or

$$
\mathcal{R}=\mathcal{R} K_{i f}(A) K_{i f}(B), \quad \text { with } \quad K_{i f}=P_{i f}^{\text {hel. }} \exp \left(i \pi\left[J_{l}(i)-J_{l}(f)\right]\right) .
$$

In terms of observables,

$$
\langle\mathcal{O}\rangle=\left\langle\left[K_{i f}(A) K_{i f}(B) \mathcal{O}^{\mathrm{pt}}\right]^{\mathrm{pt}}\right\rangle,
$$

where $p t$ means partial transposition, applied to final-particle observables only. For a spin $1 / 2$, $K_{i f}=P_{i f}^{\text {hel. }} \sigma_{z}(i) \sigma_{z}(f)=\frac{1}{2}\left[1-\sigma_{x}(i) \sigma_{x}(f)-\sigma_{y}(i) \sigma_{y}(f)+\sigma_{z}(i) \sigma_{z}(f)\right]$.

For the $1 / 2+1 / 2 \rightarrow 1 / 2+1 / 2$ case, the classical relations (2.139-2.140) lead to simple relations like $(x z \mid y 0)=-(y 0 \mid x z)$. The non-classical constraints (2.142) or (2.143) $\mathrm{read}^{7}$

$$
(\lambda \mu \mid \nu \tau)=\Gamma_{\lambda \lambda^{\prime} \nu \nu^{\prime}}^{T}\left(\lambda^{\prime} \mu^{\prime} \mid \nu^{\prime} \tau^{\prime}\right) \Gamma_{\mu \mu^{\prime} \tau \tau^{\prime}}^{T},
$$

with $\Gamma_{\alpha \beta \gamma \delta}^{T}=(1 / 4) \operatorname{Tr}\left\{\sigma_{\alpha} \sigma_{\beta} \sigma_{z} \sigma_{\gamma}^{t} \sigma_{\delta}^{t} \sigma_{z}\right\}$. Alternatively, one may keep the formulas (2.1192.120) but transforming the $(\lambda \mu \mid \nu \tau)$ 's according to

$$
(\lambda \mu \mid \nu \tau) \rightarrow(\lambda \nu \mid \check{\mu} \check{\tau}) \equiv\left\langle\sigma_{\lambda} \sigma_{\nu} \check{\sigma}_{\mu} \check{\sigma}_{\tau}\right\rangle,
$$

with $\check{\sigma}_{\alpha}=\sigma_{z} \sigma_{\alpha}^{t} \sigma_{z}$, more explicitly $\left\{\check{\sigma}_{0}, \check{\sigma}_{x}, \check{\sigma}_{y}, \check{\sigma}_{z}\right\}=\left\{\sigma_{0},-\sigma_{x}, \sigma_{y}, \sigma_{z}\right\}$. For instance, the fourth Eq. (2.120) becomes $-(0 x \mid 0 x)+(0 y \mid 0 y)+(0 z \mid 0 z)=-(x 0 \mid x 0)+(y 0 \mid y 0)+(z 0 \mid z 0)$ (with $\{x, y, z\} \equiv\{m, n, l\}$ again).

For the simpler case $1 / 2+0 \rightarrow 1 / 2+0$, for instance $\mathrm{p}+\pi \rightarrow \mathrm{p}+\pi$, one should remove the $\mu$ and $\tau$ variables and the second $\Gamma^{T}$ in (2.144). From (2.139-2.140) and (2.144), one obtains

$$
\begin{gathered}
(0 \mid m)=-(m \mid 0), \quad(0 \mid n)=+(n \mid 0), \quad(0 \mid l)=+(l \mid 0), \\
(m \mid n)=-(n \mid m), \quad(m \mid l)=-(l \mid m), \quad(n \mid l)=+(n \mid l), \\
(0 \mid 0)=(l \mid l)-(m \mid m)+(n \mid n),
\end{gathered}
$$

the last constraint being non-classical. These constraints can also be obtained applying (2.138) and (2.141) to the cross section matrix given in Table 1, with $a=\alpha_{i}, c=\alpha_{f}$.

\subsubsection{Hermitian scattering matrix}

In the Born approximation, $\mathcal{M}=\mathcal{M}^{\dagger}$. This is also the case when the intermediate states in the unitarity relation $\mathcal{M}-\mathcal{M}^{\dagger} \propto i \mathcal{M} \mathcal{M}^{\dagger}$ are kinematically forbidden, for instance in the quark-distribution amplitudes $h \rightarrow q+X$ or in the Weisszäcker-Williams amplitude for $\mathrm{e}^{-} \rightarrow \gamma+\mathrm{e}^{-}$. Here we consider the combination of hermiticity of $\mathcal{M}$ and $P T$-invariance, which gives

$$
\left\langle i^{\prime}, f^{\prime}|\mathcal{R}| i, f\right\rangle=\left\langle P T(i), P T(f)|\mathcal{R}| P T\left(i^{\prime}\right), P T\left(f^{\prime}\right)\right\rangle .
$$

Using the helicity states transformation,

$$
P T|\theta, \phi, \lambda\rangle=\eta \xi(-1)^{s+\lambda}|\theta, \phi,-\lambda\rangle,
$$

we obtain for the CSM

$$
\left\langle\lambda_{1}^{\prime}, \lambda_{2}^{\prime} ; \lambda_{3}^{\prime}, \ldots|\mathcal{R}| \lambda_{1}, \lambda_{2} ; \lambda_{3}, \ldots\right\rangle=\prod_{i}(-1)^{\lambda_{i}-\lambda_{i}^{\prime}}\left\langle-\lambda_{1},-\lambda_{2} ;-\lambda_{3}, \ldots|\mathcal{R}|-\lambda_{1}^{\prime},-\lambda_{2}^{\prime} ;-\lambda_{3}^{\prime}, \ldots\right\rangle,
$$

\footnotetext{
${ }^{7}$ This is equivalent to Eq. (4) of Appendix 3.D of [1], except for a discrepancy for the factor (1/4) in front of $\Gamma^{T}$.
} 
and for the observables

$$
\left\langle\mathcal{O}_{i} \mathcal{O}_{f}\right\rangle=\left\langle\mathcal{O}_{i}^{P T} \mathcal{O}_{f}^{P T}\right\rangle
$$

where $\mathcal{O}^{P T}$, for one-particle observables, is given by $\left\langle\lambda\left|\mathcal{O}^{P T}\right| \lambda_{1}^{\prime}\right\rangle=(-1)^{\lambda-\lambda^{\prime}}\left\langle-\lambda^{\prime}|\mathcal{O}|-\lambda\right\rangle$. For spin $1 / 2, \sigma^{P T}=-\sigma$.

This result is valid for non-planar reactions as well and any number of particles. Equation (2.151) tells that asymmetries involving an odd number of vector polarisations vanish under the $P T+$ hermiticity hypothesis. Therefore, a non-zero asymmetry is due either to $P T$ violation or to intermediate states in unitarity relation, or both. Interesting exceptions are the Sivers [68] and BoerMulders [69] effects, which are $(n \mid 0)$ and $(0 \mid n)$ asymmetries in $\vec{N} \rightarrow \vec{q}+X$. In fact, final- or initial-state interaction [33] results in an effective $h \rightarrow q+X$ amplitude which has a spin-dependent phase. Within QCD, one cannot isolate the quark emission process in a gauge invariant way. To satisfy gauge invariance, one has to include the interaction of the hard-scattered quark with spectator partons.

\subsubsection{Crossed reactions}

Let us consider the two crossed reactions:

$$
\text { (1) } X+A(\tilde{p}, \alpha) \rightarrow Y, \quad(2) \quad X \rightarrow \bar{A}(\tilde{p}, \beta)+Y,
$$

where $X$ and $Y$ represent the remaining sets of initial and final particles. $\tilde{p}$ is the four-momentum of particle $A$ or $\bar{A}$. Crossing symmetry can be formally written as

$$
\left\langle s_{Y}\left|\mathcal{M}_{1}\left(\tilde{k}_{X},-\tilde{p} ; \tilde{k}_{Y}\right)(P T)_{A}\right| s_{X}, \alpha\right\rangle=\left\langle\alpha, s_{Y}\left|\mathcal{M}_{2}\left(\tilde{k}_{X} ; \tilde{p}, \tilde{k}_{Y}\right)\right| s_{X}\right\rangle .
$$

$(P T)_{A}$ is the product of parity and time reversal operators acting on particle $A$ only. It reverses the spin of $A$ but not the momentum. $\tilde{k}_{X}, \tilde{k}_{Y}, s_{X}$ and $s_{Y}$ stand for the 4-momenta and spins of the remaining particles. The left-hand side amplitude is evaluated at non-physical kinematical variables, but can defined by analytic continuation. This equation has a simple interpretation in the Dirac hole theory, at least in the fermionic case: the annihilation of $A$ in the negative energy state $P T|-\tilde{p}, \alpha\rangle$ is equivalent to the creation of $\bar{A}$ in the state $|\tilde{p}, \alpha\rangle$. For the CSM (Eq. 2.68), the crossing relation reads

$$
\mathcal{R}_{2}\left(\tilde{k}_{X} ; \tilde{p}, \tilde{k}_{Y}\right)=(P T)_{A}^{-1} \mathcal{R}_{1}\left(\tilde{k}_{X},-\tilde{p} ; \tilde{k}_{Y}\right)(P T)_{A} .
$$

The single-spin observables $\left\langle\mathcal{O}_{A}\right\rangle_{1}$ of (1) and $\left\langle\mathcal{O}_{\bar{A}}\right\rangle_{2}$ of (2) are related by

$$
\left\langle\mathcal{O}_{\bar{A}}\left(\tilde{k}_{X} ; \tilde{p}, \tilde{k}_{Y}\right)\right\rangle_{2}=\left\langle(P T)_{A} \mathcal{O}_{A}^{t}\left(\tilde{k}_{X},-\tilde{p} ; \tilde{k}_{Y}\right)(P T)_{A}^{-1}\right\rangle_{1} .
$$

These relations can be iterated to cross several particles, for instance from $\pi^{-} \mathrm{p} \rightarrow \pi 0 \mathrm{n}$ to $\overline{\mathrm{n}} \mathrm{p} \rightarrow$ $\pi^{+} \pi 0$. Relation (2.155) also generalises to multi-particle observables: in the right-hand side the transposition of $\mathcal{O}$ concerns only the crossed particles. The crossing transformation is given below for the operators defined in this section:

$$
\begin{aligned}
& \mathcal{O} \rightarrow P T \mathcal{O}^{t}(P T)^{-1} \\
& \text { Spin 1/2: } \quad \boldsymbol{\sigma} \rightarrow-\boldsymbol{\sigma}, \\
& \text { Photon, gluon : }\left(\sigma_{\odot}, \sigma_{\oslash}, \sigma_{\ominus}\right) \rightarrow\left(-\sigma_{\odot}, \sigma_{\oslash}, \sigma_{\ominus}\right) \text {. } \\
& \text { Vector particle - Cartesian operators: } \quad\left(\Sigma_{1}, \Sigma_{2}, \Sigma_{3}\right) \rightarrow-\left(\Sigma_{1}, \Sigma_{2}, \Sigma_{3}\right) \text {, } \\
& \left(\Sigma_{4}, \cdots \Sigma_{8}\right) \rightarrow\left(\Sigma_{4}, \cdots \Sigma_{8}\right), \\
& \tau_{2 \mu} \rightarrow \tau_{2 \mu} \text {. }
\end{aligned}
$$$$
\text { Vector particle - magnetic operators: } \quad \mathcal{S}_{\lambda} \rightarrow-\mathcal{S}_{\lambda}
$$

As an example of application of (2.155), the quark transversity correlation in $\mathrm{e}^{+} \mathrm{e}^{-} \rightarrow q \bar{q}$ is $(00 \mid n n)=\left\langle\sigma_{n}(q) \sigma_{n}(\bar{q})\right\rangle=-2 t u /(t 2+u 2)$. The crossed observable in $\mathrm{e}^{-} q \rightarrow \mathrm{e}^{-} q$ is the 
depolarisation parameter $(0 n \mid 0 n)=\left\langle\sigma_{n}\left(q_{i}\right) \sigma_{n}\left(q_{f}\right)\right\rangle=2 s u /(s 2+u 2)$, which can be obtained from $(00 \mid n n)$ by crossing $t \rightarrow s$ and a change of sign owing to (2.156).

If one knows constraints for the observables of $A+B \rightarrow C+D(+X)$, one can obtain analogous constraints for $A+\bar{C} \rightarrow \bar{B}+D(+X)$, using the crossing relations (2.156). For instance, in the inclusive case (see Sec. 4), Eq. (4.22) can be deduced from Eq. (4.11).

\subsubsection{Chiral invariance}

In hard scattering processes, helicity is conserved along a fermion line, up to correction factors of the order of $\left|m^{2} / \tilde{q}^{2}\right|^{1 / 2}$, where $\tilde{q}=\tilde{p}^{\prime}-\tilde{p}$ is the four-momentum transferred to this line by the other particles. In an annihilation or creation process, $\tilde{q}$ is time-like and the helicities of the fermion and the antifermion add up to zero. This conservation rule comes from the chiral invariance of the Lagrangian of the standard model in the $m \rightarrow 0$ limit. Thus, in this limit, if fermions $f_{1}$ and $f_{2}$ or $\bar{f}_{2}$ are on the same line, $D_{l l}=+1$ in the $f_{1} \rightarrow f_{2}$ case and $A_{l l}$ or $C_{l l}=-1$ in the $f_{1}+\bar{f}_{2}$ case.

In many cases, however, different Feynman diagrams connect external particles by fermion lines in different ways. For instance in $\mathrm{e}^{+} \mathrm{e}^{-} \rightarrow \mathrm{e}^{++} \mathrm{e}^{\prime-}$, the $t$-channel pole diagram connects $\mathrm{e}^{-}$to $\mathrm{e}^{\prime-}$ whereas the $s$-channel pole connects $\mathrm{e}^{-}$to $\mathrm{e}^{+}$. Let us first consider a process

$$
X \rightarrow f_{1}+f_{2} \ldots+f_{N}+Y,
$$

where the spin- $1 / 2$ fermions $f_{1}, \ldots f_{N}$ are not connected to the remaining particles, which are globally denoted as $X$ and $Y$. The longitudinally polarised cross section has the form

$$
\sigma\left(\lambda_{1}, \lambda_{2}, \ldots \lambda_{N}\right)=I_{0}\left(1+A_{1} \lambda_{1}+A_{2} \lambda_{2}+\ldots+A_{N} \lambda_{N}+A_{12} \lambda_{1} \lambda_{2}+\ldots\right),
$$

where $\lambda= \pm 1$ is twice the helicity. Due to helicity conservation, this cross section is non-vanishing only if $\lambda_{1}+\lambda_{2}+\cdots+\lambda_{N}=0$, which can be expressed by

$$
\left(\lambda_{1}+\lambda_{2}+\cdots+\lambda_{N}\right) \sigma\left(\lambda_{1}, \lambda_{2}, \ldots, \lambda_{N}\right)=0 .
$$

Before using this identity, one must replace every $\lambda_{i}^{2}$ by 1 . Then the coefficient of each monomial, e.g.of $\lambda_{2} \lambda_{5} \lambda_{7}$, has to vanish. On gets the following sets of identities

$$
\begin{aligned}
A_{1}+A_{2}+\cdots+A_{N}=0 & \\
1+A_{12}+A_{13}+\cdots+A_{1 N}=0 & \text { and permutations, } \\
A_{1}+A_{2}+A_{123}+A_{124}+\cdots+A_{12 N}=0 & \text { and permutations, } \\
A_{12}+A_{23}+A_{13}+A_{1234}+A_{1235}+\cdots+A_{123 N}=0 & \text { and permutations, }
\end{aligned}
$$

and so on. The first equation tells that the average total final helicity is zero. The second tells that if particle 1 has $\lambda=+1$, the average total helicity of the remaining particles is -1 , etc. (these equations take simpler homogeneous forms if one defines $A_{11}=1, A_{121}=A_{2}, A_{1231}=A_{23}$, etc.).

Identities for the more general reaction

$$
f_{1}+f_{2} \ldots+f_{M}+X \rightarrow f_{M+1} \ldots+f_{N}+Y
$$

are obtained from the preceding one by crossing, using (2.156). Thus, in $\mathrm{e}^{+} \mathrm{e}^{-} \rightarrow \mathrm{e}^{+} \mathrm{e}^{-}$at large momentum transfer, we have $(l 0 \mid 00)+(0 l \mid 00)=(00 \mid l 0)+(00 \mid 0 l), 1+(l l \mid 00)=(l 0 \mid l 0)+(l 0 \mid 0 l)$, etc.

Chiral invariance has also major consequences on transverse spin observables. First of all, the CSM matrix $\left\langle\lambda_{1}, \ldots \lambda_{N}|\mathcal{R}| \lambda_{1}, \ldots \lambda_{N}\right\rangle$ of (2.164) cannot contain an odd number of helicity flips $\lambda_{i}^{\prime}=$ $-\lambda_{i}$. Therefore $\left\langle\mathcal{O}_{\lambda \mu \ldots} \ldots\right\rangle=0$ for an odd number of transverse indices. In particular single transverse spin asymmetries vanish, at least in a hard sub-process. The quark transversity ${ }^{8}$ distribution $\delta q(x)$

\footnotetext{
${ }^{8}$ In the parton terminology, transversity means transverse polarisation of any azimuth, i.e., not necessarily normal to the scattering plane.
} 
in the nucleon cannot be measured in ordinary deep-inelastic scattering, since it involves only one helicity flip along the quark line [70]. But it can be measured in the semi-inclusive reaction $\mathrm{e}^{-}+$ $\vec{N} \rightarrow \mathrm{e}^{--}+\vec{\Lambda}+X$ if the $\Lambda$ is analysed [71].

Transversity correlations (T.C.) between two fermions of a hard subprocess exist only if they belong to the same connected subset. In QED and QCD, there is no T.C. between quarks and leptons or between quarks of different flavour. Photons or gluons do not mediate transverse spin information. Under $Q^{2}$ evolution, $\delta q(x)$ does not mix with any polarised gluon distribution, unlike $\Delta q(x)$. Note that T.C. can exist between two fermions $f_{1}$ and $f_{2}$ not directly connected in any Feynman diagram, for instance the initial $\mathrm{e}^{-}$and the final $\mathrm{e}^{+}$of Bhabha scattering. It suffices that a third fermion $f_{3}$ connects to $f_{1}$ in a diagram $D_{1}$ and to $f_{2}$ in a diagram $D_{2}$. In fact $f_{1}$ and $f_{2}$ are connected in the unitarity diagram representing the $D_{1} \times D_{2}$ interference.

The azimuthal dependence of allowed transverse spin correlations are strongly constrained as we shall see below. In the massless limit, longitudinal polarisation $\boldsymbol{S}_{\mathrm{L}}$ is a Lorentz pseudoscalar and transverse polarisation $S_{\mathrm{T}}$ can be promoted to a four-vector $\mathcal{T}^{\mu}$ defined up to a "gauge" transformation [72]

$$
\mathcal{T}^{\mu} \rightarrow \mathcal{T}^{\mu}+C p^{\mu}
$$

and constrained by $\tilde{p} \cdot \mathcal{T}=0$ and $\boldsymbol{S}_{\mathrm{L}}^{2}-\mathcal{T}^{2} \equiv \boldsymbol{S}_{\mathrm{L}}^{2}+\boldsymbol{S}_{\mathrm{T}}^{2} \leq 1$. The "gauge" $\mathcal{T}=\left(0, \boldsymbol{S}_{\mathrm{T}}\right)$ is generally used. A pure state with $\boldsymbol{S}_{\mathrm{T}} \neq 0$ can be expressed, up to an over-all phase, as the following superposition of helicity states

$$
\left|\boldsymbol{S}_{\mathrm{L}}, \boldsymbol{S}_{\mathrm{T}}\right\rangle=\left|\frac{\boldsymbol{S}_{\mathrm{T}}}{2}\right|^{\frac{1}{2}}\left[\exp \left(\frac{\eta-i \phi}{2}\right)\left|+\frac{1}{2}\right\rangle+\exp \left(\frac{-\eta+i \phi}{2}\right)\left|-\frac{1}{2}\right\rangle\right],
$$

where $\phi$ is the azimuth of $\boldsymbol{S}_{\mathrm{T}}$ in the $\{\boldsymbol{m}, \boldsymbol{n}, \boldsymbol{l}\}$ frame of the particle, $\boldsymbol{S}_{\mathrm{L}} \equiv \tanh \eta$ and $\left|\boldsymbol{S}_{\mathrm{T}}\right|=$ $1 / \cosh \eta$. The chiral transformation $\Psi \rightarrow \exp \left(-i \Delta \phi \gamma_{5} / 2\right) \Psi$ multiplies the right-handed component by $\exp (-i \Delta \phi / 2)$ and the left-handed one by $\exp (i \Delta \phi / 2)$, therefore rotates $\boldsymbol{S}_{\mathrm{T}}$ by $\Delta \phi$ about the momentum.

Applying chiral symmetry to the reaction (2.164), the polarised cross section is invariant under the simultaneous rotation of the transversity vectors of $f_{1}, \ldots f_{N}$ by a common angle $\Delta \phi$ about the respective particle momenta. We can call this a "Cardan transformation", by analogy with rotations transmitted by a mechanical shaft drive transmission. In $\mathrm{e}^{-} q \rightarrow \mathrm{e}^{-} q$, for instance, where the quarks are on the same fermion line, the "Cardan" invariance gives $D_{n n}=D_{m m}, D_{m n}=-D_{n m}$. In the inclusive section $f_{a}+f_{b} \rightarrow C+X$, one has $A_{n n}=A_{m m}, A_{m n}=-A_{n m}$. No transverse spin correlation survives if one integrates over the azimuth of particle $C$ (Hikasa theorem [73]). Indeed, an anticlockwise rotation of the scattering plane about $\boldsymbol{p}_{a}$ is equivalent to a clockwise rotation of both $\boldsymbol{S}_{\mathrm{T}}(a)$ and $\boldsymbol{S}_{\mathrm{T}}(b)$ about $\boldsymbol{p}_{a}$, which is at variance with Cardan transformation (clockwise rotations of $\boldsymbol{S}_{\mathrm{T}}(a)$ and $\boldsymbol{S}_{\mathrm{T}}(b)$ about $\boldsymbol{p}_{a}$ and $\boldsymbol{p}_{b}$ respectively).

In a similar manner, we can apply the imaginary chiral rotation $\exp \left(\Delta \eta \gamma_{5}\right)$ to initial fermions and $\exp \left(-\Delta \eta \gamma_{5}\right)$ to the final fermion, that is to say

$$
\eta_{i} \rightarrow \eta_{i}+\Delta \eta \quad \text { for initial fermions }, \quad \eta_{j} \rightarrow \eta_{j}-\Delta \eta \text { for final fermions. }
$$

This "see-saw" transformation leaves invariant the rescaled cross section

$$
\tilde{\sigma} \equiv \sigma \prod_{1}^{N}\left|\boldsymbol{S}_{\mathrm{T}}(i)\right|^{-1}
$$

\subsubsection{Inequalities relating isospin-partner reactions}

Consider for instance the nucleon-antinucleon elastic and charge-exchange scattering reactions, in the limit of isospin symmetry. The isospin $I=1$ amplitude $\mathcal{M}_{1}$ corresponds to $\overline{\mathrm{n}} \mathrm{p} \rightarrow \overline{\mathrm{n}} \mathrm{p}$, while $\overline{\mathrm{p}} \mathrm{p}$ elastic scattering is governed by the combination $\left(\mathcal{M}_{1}+\mathcal{M}_{0}\right) / 2$ of $I=1$ and $I=0$, and the charge 
exchange (c.e.) $\overline{\mathrm{p} p} \rightarrow \overline{\mathrm{n}}$, to $\left(\mathcal{M}_{1}-\mathcal{M}_{0}\right) / 2$ [25]. Each isospin amplitude $\mathcal{M}_{i}$ includes five spin amplitudes (this is a limiting case of the $\bar{p} p \rightarrow \bar{\Lambda} \Lambda$, with $g \rightarrow 0$ when the baryon masses become equal, see Sec. 3.3 for details), and can be written as

$$
(a+b) \mathbb{1}+(a-b) \boldsymbol{\sigma}_{1} . \boldsymbol{n} \boldsymbol{\sigma}_{2} . \boldsymbol{n}+(c+d) \boldsymbol{\sigma}_{1} \cdot \boldsymbol{m} \boldsymbol{\sigma}_{2} \cdot \boldsymbol{m}+(c-d) \boldsymbol{\sigma}_{1} . \boldsymbol{l} \boldsymbol{\sigma}_{2} . \boldsymbol{l}+i e\left(\boldsymbol{\sigma}_{1}+\boldsymbol{\sigma}_{2}\right) \cdot \boldsymbol{n} .
$$

Hence,

$$
a(\overline{\mathrm{n}} \mathrm{p})=a(\overline{\mathrm{p}} \mathrm{p})+a(\text { c.e. }),
$$

and similarly for the four other amplitudes $b, c, d, e$ entering $\mathcal{M}$, as per Eq. (2.169).

Since $I_{0}=|a|^{2}+|b|^{2}+\cdots|f|^{2}$, it is a textbook exercise to derive from Eq. (2.170)

$$
\left.[\sqrt{\sigma(\overline{\mathrm{p}} \mathrm{p}})-\sqrt{\sigma(\text { c.e. })}]^{2} \leq \sigma(\overline{\mathrm{n}} \mathrm{p}) \leq[\sqrt{\sigma(\overline{\mathrm{p}} \mathrm{p}})+\sqrt{\sigma(\text { c.e. })}\right]^{2}
$$

either for the angular distribution $I_{0}$ or the integrated cross-section $\sigma$.

However, it is less known that inequalities can also be derived for spin observables. At first sight, nothing simple can be written for, e.g., the polarisation $P$ and analysing power $A_{n}$ given by $I_{0} P=I_{0} A_{n}=2 \Im \mathrm{m}\left(a e^{*}\right)$. If it is rewritten in the transversity basis as $I_{0} P=\left|a^{\prime}\right|^{2}-\left|e^{\prime}\right|^{2}$, it is readily seen that the inequality of type (2.171) written for $\left|a^{\prime}\right|^{2}$ cannot be safely combined with those written for $\left|e^{\prime}\right|^{2}$. The difficulty was overcome by Michel et al. [1,74] (see, also, [75-78]). The quantities $I_{0}(1 \pm P)$ are easily seen to consist of a sum of positive terms in the transversity basis

$$
I_{0}(1-P)=\left|b^{\prime}\right|^{2}+\left|c^{\prime}\right|^{2}+\left|d^{\prime}\right|^{2}+2\left|e^{\prime}\right|^{2}, \quad I_{0}(1+P)=2\left|a^{\prime}\right|^{2}+\left|b^{\prime}\right|^{2}+\left|c^{\prime}\right|^{2}+\left|d^{\prime}\right|^{2} .
$$

Hence both quantities $X_{\mp}=I_{0}(1 \mp P)$ verify

$$
[\sqrt{X(\overline{\mathrm{p}} \mathrm{p})}-\sqrt{X(\text { c.e. })}]^{2} \leq X(\overline{\mathrm{n}} \mathrm{p}) \leq[\sqrt{X(\overline{\mathrm{p}} \mathrm{p})}+\sqrt{X(\text { c.e. })}]^{2} .
$$

This result is rather general. For any spin observable $\mathcal{O}$, there always exists a basis for the amplitudes such that

$$
I_{0} \mathcal{O}= \pm|a|^{2} \pm|b|^{2}+\cdots
$$

so that $I_{0}(1 \pm \mathcal{O})$ is a sum of squared amplitudes. 


\section{Exclusive reactions}

In this section, we review the formalism for some exclusive reactions, with various number of amplitudes and spin observables. More details are given for the hyperon-pair production reaction $\overline{\mathrm{p}} \mathrm{p} \rightarrow \bar{\Lambda} \Lambda$ and for the photoproduction of pseudoscalar mesons, for which measurements have been performed recently.

\subsection{Pion-nucleon elastic scattering}

As described in many textbooks, see, e.g., [79], there are two independent amplitudes $\mathcal{A}(s, t)$ and $\mathcal{B}(s, t)$ to describe $\pi \mathrm{N}$ elastic scattering. They are defined by

$$
\mathcal{M}_{\mathrm{fi}}=-2 m \bar{u}\left(\tilde{p}^{\prime}\right)\left[-\mathcal{A}+i \gamma \cdot \frac{\tilde{q}+\tilde{q}^{\prime}}{2} \mathcal{B}\right] u(\tilde{p}),
$$

acting between the Dirac spinors of initial and final nucleons of four-momenta $\tilde{p}$ and $\tilde{p}^{\prime}$, respectively, while $\tilde{q}$ and $\tilde{q}^{\prime}$ are the pion four-momenta. The amplitude can be rewritten in the centre-of-mass as

$$
\mathcal{M}_{\mathrm{fi}}=8 \pi \sqrt{s} \chi_{f}^{\dagger}(f+i g \boldsymbol{\sigma} . \boldsymbol{n}) \chi_{i},
$$

acting on the Pauli spinors $\chi_{i}$ and $\chi_{f}$, with the relations [79]

$$
f=\frac{p^{0}+m}{8 \pi \sqrt{s}}[\mathcal{A}+\mathcal{B}(\sqrt{s}-m)], \quad g=-\frac{p^{0}-m}{8 \pi \sqrt{s}}[\mathcal{A}-\mathcal{B}(\sqrt{s}+m)],
$$

where the normalisation is such that the angular distribution (differential cross section) reads

$$
\frac{\mathrm{d} \sigma}{\mathrm{d} \Omega}=I_{0}=|f|^{2}+|g|^{2} .
$$

The first spin observable is the polarisation $P_{n}$ of the recoiling nucleon. It is equal to the analysing power $A_{n}$, accessible if the target is transversally polarised. They are given by

$$
I_{0} P_{n}=I_{0} A_{n}=2 \Im \mathrm{m}\left(f g^{*}\right) .
$$

Most phase-shift analyses, which led to valuable information on pion dynamics and nucleon resonances were based on the data available on $I_{0}$ and $P_{n}$. See, e.g., [80] and Refs. therein.

Two additional observables are actually measurable if the target is polarised. The scattering process conserves the polarisation modulus, since the pion carry no spin information, and rotates the $(\boldsymbol{n}, \boldsymbol{S})$ plane by an angle $\alpha$ about $\boldsymbol{n}$ (here $\boldsymbol{S}^{\prime}$ stands for $\left\langle\boldsymbol{S}^{\prime}\right\rangle$ in the notation of (2.7)). If all spins are measured in the frame $\left\{\boldsymbol{l}_{\mathrm{b}}=-\hat{\boldsymbol{p}}, \boldsymbol{m}_{\mathrm{b}}, \boldsymbol{n}\right\}$ associated with the beam, the depolarisation parameters $D_{i j}=C_{i 0 j 0}$ which do not vanish are

$$
\left.D_{n n}^{(\mathrm{b})}=1, \quad D_{m m}^{(\mathrm{b})}=D_{l l}^{(\mathrm{b})}=(\mid f]^{2}-|g|^{2}\right) / I_{0}, \quad D_{m l}^{(\mathrm{b})}=-D_{l m}^{(\mathrm{b})}=2 \Re \mathrm{e}\left(f g^{*}\right) / I_{0} .
$$

These parameters fulfil the Bohr identity (2.100). If each particle has its spin measured is its own frame, this gives the so-called spin-rotation parameters $A=D_{l l}=D_{m m}$ and $R=D_{l m}=-D_{m l}$, given by

$$
\begin{aligned}
& I_{0} A=\left(|f|^{2}-|g|^{2}\right) \cos \theta-2 \Re \mathrm{e}\left(f g^{*}\right) \sin \theta, \\
& I_{0} R=-\left(|f|^{2}-|g|^{2}\right) \sin \theta-2 \Re \mathrm{e}\left(f g^{*}\right) \cos \theta .
\end{aligned}
$$

From Eqs. (3.5) and (3.7), it is obvious that

$$
P_{n}^{2}+A^{2}+R^{2}=1
$$

This can be interpreted as follows: given a purely sideways or purely longitudinal target polarisation, the sum of the square of the three components of the polarisation of the final nucleon is unity. In 
Table 2: Spin observables for $\pi^{-}$p elastic scattering, as measured by Abaev et al. [81] at 0.573 and $0.685 \mathrm{GeV} / c$.

\begin{tabular}{cccrr}
$p_{\text {lab }}$ & $\cos \left(\vartheta_{\mathrm{cm}}\right)$ & $R$ & \multicolumn{1}{c}{$A$} & \multicolumn{1}{c}{$P_{n}$} \\
\hline & -0.63 & $0.47 \pm 0.22$ & $0.53 \pm 0.20$ & $0.70 \pm 0.13$ \\
0.573 & -0.72 & $0.73 \pm 0.11$ & $-0.03 \pm 0.26$ & $0.68 \pm 0.11$ \\
& -0.80 & $0.82 \pm 0.07$ & $0.01 \pm 0.23$ & $0.56 \pm 0.10$ \\
& -0.63 & $0.86 \pm 0.05$ & $0.16 \pm 0.14$ & $-0.49 \pm 0.07$ \\
0.685 & -0.72 & $0.87 \pm 0.04$ & $0.19 \pm 0.12$ & $-0.46 \pm 0.07$ \\
& -0.80 & $0.86 \pm 0.06$ & $-0.13 \pm 0.20$ & $-0.50 \pm 0.08$ \\
\hline
\end{tabular}
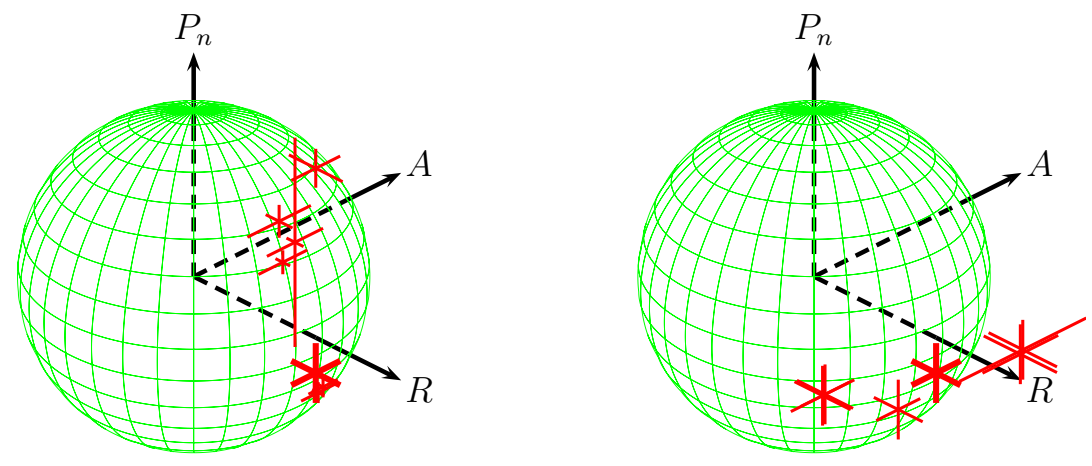

Figure 6: Spin observables for $\pi^{-}$p elastic scattering at 0.573 and $0.685 \mathrm{GeV} / c$ (left), and for $\pi^{+} \mathrm{p}$ elastic scattering at $0.657 \mathrm{GeV} / c$ (right), in axes $\left\{R, A, P_{n}\right\}$ compared to the unit sphere. The data are from Abaev et al. [81] for $\pi^{-} \mathrm{p}$ and Supek et al. [82] for $\pi^{+} \mathrm{p}$, and are shown with the error bars on the three observables.

practice, this means that two of these observables are needed, as well as the sign of the third one, for a complete information.

A value $D_{m m}^{(\mathrm{b})}=0$, for instance, indicates that $|f|=|g|$, but does not inform about the relative phase of the two amplitudes. If, instead, it happens that $D_{m m}^{(\mathrm{b})}=+1$, then $g=0$, and the measurement of $D_{m l}^{(\mathrm{b})}$ or $P_{n}$ is not necessary, except for cross-checking.

Results for the $\pi \mathrm{N}$ observables are listed in the Durham data base of the Particle Data Group. Note that in some cases, the constraint (3.8) is imposed in the analysis. In Tables 2 and 3, examples are given of simultaneous measurements of $A, R$ and $P_{n}$. The corresponding data are plotted in $A$, $R, P_{n}$ coordinates in Fig. 6, against the unit sphere. The constraint (3.8) is reasonably satisfied with regard to the error bars.

The reactions $\mathrm{K}^{ \pm} \mathrm{p} \rightarrow \mathrm{K}^{ \pm} \mathrm{p}$ have the same spin algebra than $\pi^{ \pm} \mathrm{p} \rightarrow \pi^{ \pm} \mathrm{p}$. No spin transfer data are available in the case of $\mathrm{K}^{+}$. In the case of $\mathrm{K}^{-}$, some measurements have been done for $R$ at $p_{\text {lab }}=40 \mathrm{GeV}$ [83], and $A$ was computed from $A=\left(1-R^{2}-P_{n}^{2}\right)^{1 / 2}$, using the polarisation data of the same group [84]. 
Inequalities on spin observables

Table 3: Spin observables for $\pi^{+} \mathrm{p}$ elastic scattering, as measured by Supek et al. [82] at $0.657 \mathrm{GeV} / c$.

\begin{tabular}{rcrr}
$\cos \left(\vartheta_{\mathrm{cm}}\right)$ & $R$ & $A$ & $P_{n}$ \\
\hline $0.150 \pm 0.012$ & $1.04 \pm 0.30$ & $0.65 \pm 0.31$ & $-0.36 \pm 0.20$ \\
$0.079 \pm 0.012$ & $1.05 \pm 0.28$ & $0.66 \pm 0.52$ & $-0.33 \pm 0.20$ \\
$-0.704 \pm 0.008$ & $1.03 \pm 0.20$ & $-0.34 \pm 0.18$ & $-0.33 \pm 0.20$ \\
$-0.755 \pm 0.008$ & $0.68 \pm 0.23$ & $-0.61 \pm 0.26$ & $-0.27 \pm 0.20$ \\
$-0.796 \pm 0.008$ & $0.74 \pm 0.23$ & $-0.65 \pm 0.20$ & $-0.22 \pm 0.20$ \\
\hline
\end{tabular}

\subsection{Antiproton-proton annihilation into two pseudoscalar mesons}

The reaction $\overline{\mathrm{p}} \mathrm{p} \rightarrow \pi \pi$ is related by crossing to $\pi \mathrm{N}$ elastic scattering and thus has the same number of independent amplitudes. The formalism is given, e.g., by Frazer and Fulco [85]. The amplitude can be written as

$$
\mathcal{M}_{\mathrm{fi}}=-2 m \bar{u}\left(\tilde{p}^{\prime}\right)\left[-\mathcal{A}+i \gamma \cdot \frac{\tilde{q}-\tilde{q}^{\prime}}{2} \mathcal{B}\right] v(\tilde{p}),
$$

in terms of the Dirac spinors and four-momenta of the antinucleon: $\tilde{p}=\left(p^{0}, \boldsymbol{p}\right)$, nucleon $\tilde{p}^{\prime}=$ $\left(p^{0},-\tilde{p}\right)$ and pions $\tilde{q}, \tilde{q}^{\prime}=\left(q^{0}, \pm \boldsymbol{q}\right)$. It can be rewritten as

$$
\mathcal{M}_{\mathrm{fi}}=8 \pi \sqrt{s} \sqrt{\frac{p}{q}} \mathcal{H}, \quad \mathcal{H}=\chi^{\dagger}(\mathrm{N})\left(h_{1} \boldsymbol{\sigma} \cdot \boldsymbol{p}_{1}+h_{2} \boldsymbol{\sigma} \cdot \boldsymbol{p}_{2}\right) \chi(\overline{\mathrm{N}}),
$$

where $\chi$ is the spinor of the nucleon or antinucleon, and $m$ the nucleon mass. If the spinors are chosen as spin states along the initial momentum $\boldsymbol{p}_{1}$, the helicity amplitudes

$$
\mathcal{H}_{++}=h_{1} p_{1}+h_{2} p_{2} \cos \theta, \quad \mathcal{H}_{+-}=h_{2} p_{2} \sin \theta,
$$

are obtained, to which the transversity amplitudes are related by

$$
\mathcal{H}^{+}=\frac{\mathcal{H}_{++}+i \mathcal{H}_{+-}}{\sqrt{2}}, \quad \mathcal{H}^{-}=\frac{\mathcal{H}_{++}-i \mathcal{H}_{+-}}{\sqrt{2}} .
$$

The spin observables are the analysing power, the beam asymmetry and the spin correlations in the initial state, and are submitted to an identity of type (3.8). Only the differential cross-section $I_{0}$ and the analysing power $A_{n}$ have been reached, as polarised antiprotons are not yet available. They are given by

$$
\begin{aligned}
I_{0} & =\left|\mathcal{H}_{++}\right|^{2}+\left|\mathcal{H}_{+-}\right|^{2}=\left|\mathcal{H}^{+}\right|^{2}+\left|\mathcal{H}^{-}\right|^{2}, \\
I_{0} A_{n} & =2 \Im \mathrm{m}\left(\mathcal{H}_{++} \mathcal{H}_{+-}^{*}\right)=\left|\mathcal{H}^{+}\right|^{2}-\left|\mathcal{H}^{-}\right|^{2} .
\end{aligned}
$$

The analysing power $A_{n}$ has been measured by the PS172 collaboration working with the LEAR facility of CERN [86]. They got remarkable results, with $A_{n}$ saturating or nearly saturating the limit $\left|A_{n}\right| \leq 1$ in a wide domain of energies and angles. See Fig. 7 .

These results triggered several discussions. An amplitude analysis indicated the possibility of a series of broad resonances [87]. In Ref. [88], it was stressed that the PS172 results suggest a simple relation $\mathcal{M}_{++} \propto i \mathcal{M}_{+-}$between the helicity amplitudes.

In [89], it was pointed out that the transversity states are eigenstates of the tensor operator, which is expected to be very large in the nucleon-antinucleon initial state, especially for isospin $I=0$ [90]. Both eigenstates experience a strong annihilation, but if the real potential is attractive in one state 

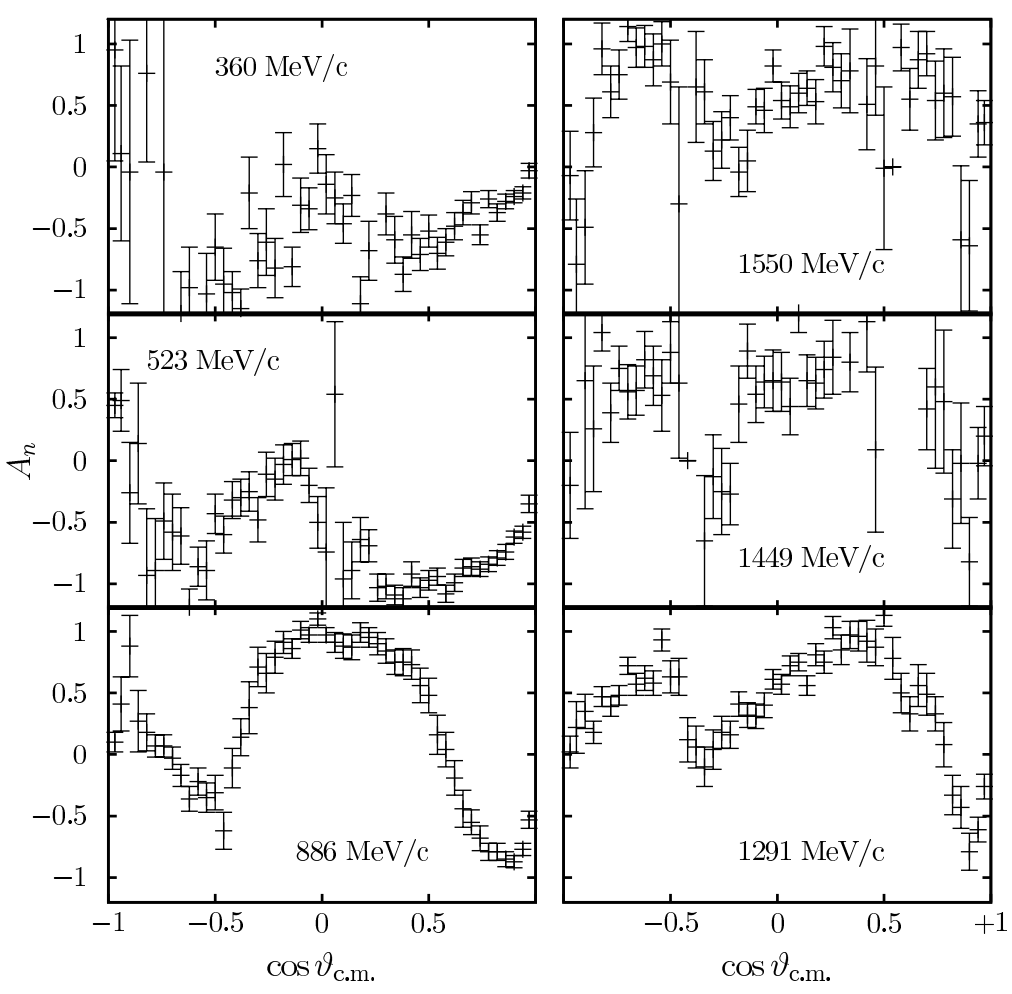

Figure 7: Analysing power for $\bar{p} p \rightarrow \pi^{-} \pi^{+}$at selected values of the antiproton momentum in the target frame. The data are from the PS172 collaboration [86].

and repulsive in the other one, the amplitudes acquire different phases, and this produces a large value for $A_{n}$, as in Eq. (3.13). Hence the strong spin effect seen by PS172 is partly due to the strong spin dependence of the nucleon-antinucleon interaction.

The PS 172 collaboration [86] also measured the analysing power for $\bar{p} \mathrm{p} \rightarrow \mathrm{K}^{+} \mathrm{K}^{-}$, and found that $\left|A_{n}\right|$ is also rather large at some angles, see Fig. 8.

If annihilation into two pseudoscalar mesons is ever measured with a polarised antiproton beam hitting a polarised target, then it could be checked that the beam asymmetry $C_{n 000}=-A_{n}$, and that the spin correlation coefficients $A_{m m}$ and $A_{m l}$ vanish when $\left|A_{n}=1\right|$. Indeed, from [2],

$$
I_{0} A_{m m}=\left|\mathcal{H}_{++}\right|^{2}-\left|\mathcal{H}_{+-}\right|^{2}, \quad I_{0} A_{m l}=-2 \Re \mathrm{e}\left(\mathcal{H}_{++} \mathcal{H}_{+-}^{*}\right),
$$

and one easily derives $A_{n}^{2}+A_{m m}^{2}+A_{m l}^{2}=1$, which is the analogue of (3.8) for the crossed reaction.

\subsection{Hyperon-pair production}

\subsubsection{Experimental situation}

The reaction $\bar{p} p \rightarrow \bar{\Lambda} \Lambda$ and other strangeness-exchange scattering processes with an antihyperonhyperon pair in the final state has been measured at various energies. The most precise and systematic study has been carried out by the PS185 collaboration [91-94] at the LEAR facility of CERN.

The aim was to study how strangeness is produced. Schematically, two classes of models existed when the PS185 experiment was proposed. The first one is based on $\mathrm{K}$ and $\mathrm{K}^{*}$ exchange, in analogy with the exchange of charged mesons such as $\pi^{+}$or $\rho^{+}$mediating the charge-exchange reaction $\overline{\mathrm{p}} \mathrm{p} \rightarrow \overline{\mathrm{n}}$. Alternatively, hyperon-pair production can be described at the quark level, with a pair of 

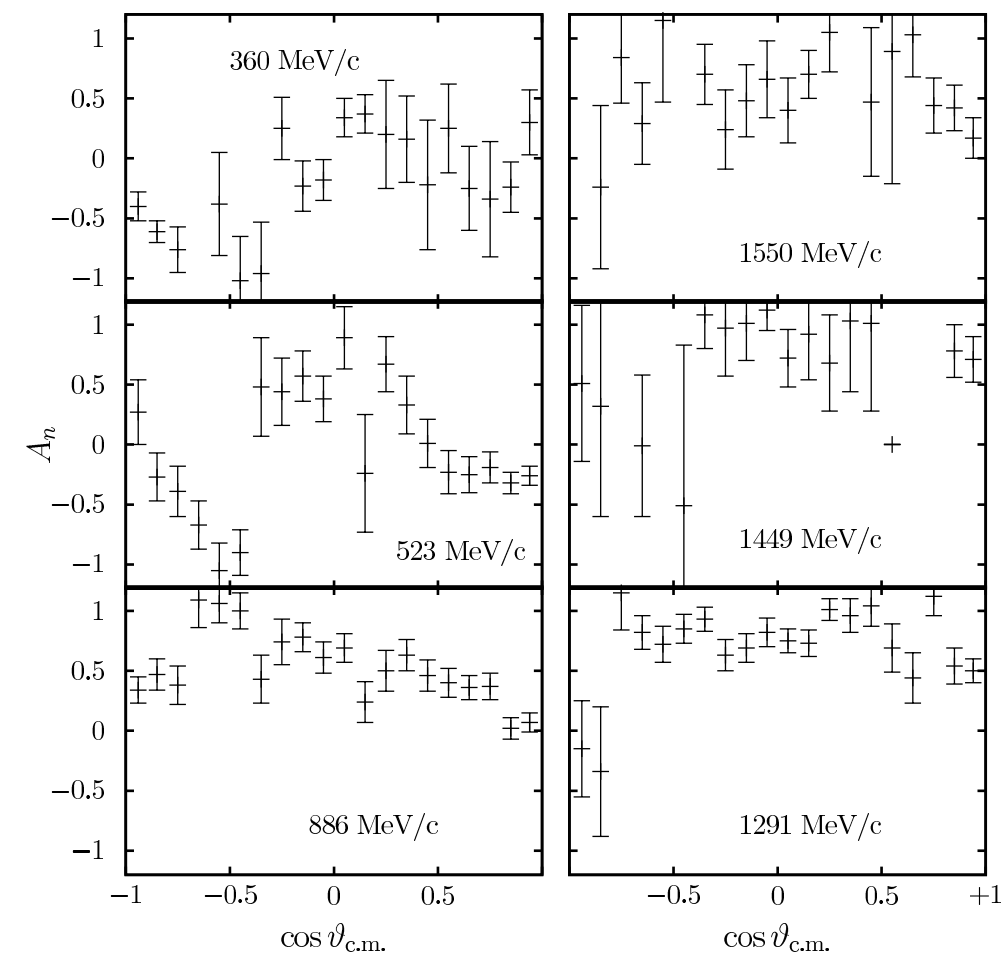

Figure 8: Analysing power for $\overline{\mathrm{p}} \mathrm{p} \rightarrow \mathrm{K}^{-} \mathrm{K}^{+}$at selected values of the antiproton momentum in the target frame. The data are from the PS172 collaboration [86].

light quarks annihilated and a pair of strange quarks, $\bar{s} s$, created out of the vacuum, for instance in the ${ }^{3} \mathrm{P}_{0}$ model [95].

Both models were rather successful to describe the first data of PS185, taken with an unpolarised proton target at various values of the incoming antiproton momentum. This corresponds to the polarisation $P_{n}$ and final-state spin-correlation coefficients $C_{i j}$, out of which the singlet fraction can be estimated. Examples of observables accessible without polarised target are given in Fig. $9^{9}$.

To better distinguish among the models, the experiment was improved as to include a polarised target. An estimate by Holinde et al. [96], for instance, indicated that the depolarisation $D_{n n}$ is predicted differently by the kaon-exchange model and the quark-pair creation model. Meanwhile a third type of model was proposed by Alberg et al. [26], in which the $\bar{s} s$ pair is not created out the vacuum, but extracted out the polarised sea of the incoming proton or antiproton, leading to a pronounced negative $D_{n n}{ }^{10}$. This is in this context that a warning was set that the existing data already constrain the allowed range of $D_{n n}[27,28]$, and the problem of the spin observables of the $\overline{\mathrm{p}} \mathrm{p} \rightarrow \bar{\Lambda} \Lambda$ reaction was further studied [29-31,97].

The PS185 data of the at incident momentum $p_{\text {lab }}=1.637 \mathrm{GeV} / c$ have been analysed and published in great detail $[91,92,94]$. Some of them are given in Figs. 9 and 10. Preliminary results of the $1.525 \mathrm{GeV} / c$ measurements have been presented at the LEAP03 conference [93], but never published, to our knowledge.

\footnotetext{
${ }^{9}$ They actually correspond to the last run, with a polarised target, but are equivalent to the earlier data obtained without polarisation of the target.

${ }^{10}$ Remember that $D_{n n}=+1$ in absence of spin-dependent forces.
} 


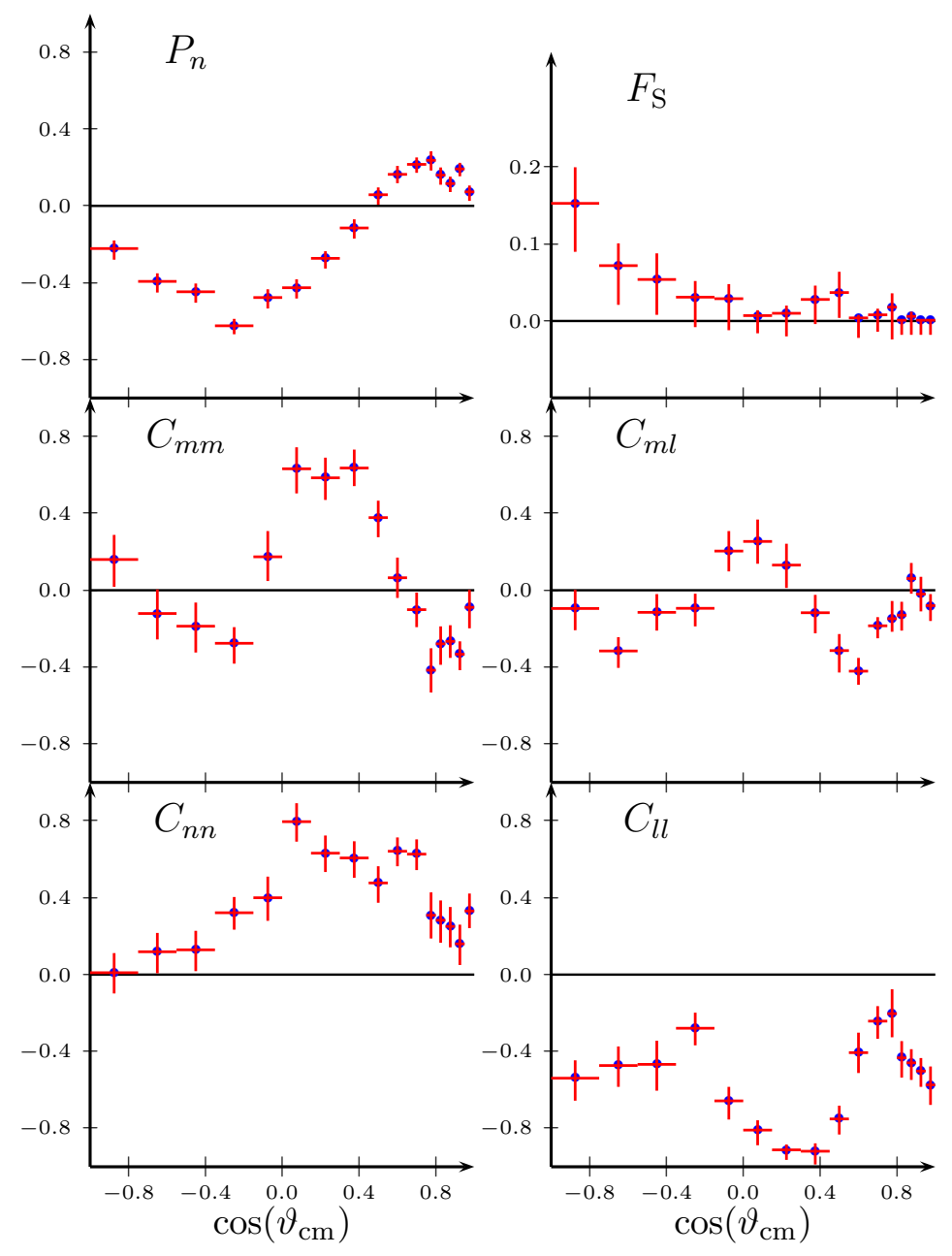

Figure 9: Results of PS 185 [94] on $\bar{p} p \rightarrow \bar{\Lambda} \Lambda$ for the polarisation, and initial-state spin correlations, out of which the spin-singlet fraction $F_{S}$ can be estimated. This corresponds to one of the last runs, at $p_{\text {lab }}=1.637 \mathrm{GeV} / c$. Earlier measurements, obtained without polarised target, are in good agreement.

\subsubsection{Formalism of hyperon-pair production}

There are six independent amplitudes for $\bar{p} p \rightarrow \bar{\Lambda} \Lambda$, once symmetries are enforced. We follow here the notation adopted in the recent literature. See, for example [98-100]. The amplitude is denoted

$$
\begin{aligned}
\mathcal{M}=(a+b) \mathbb{1} & +(a-b) \boldsymbol{\sigma}_{1} \cdot \boldsymbol{n} \boldsymbol{\sigma}_{2} \cdot \boldsymbol{n}+(c+d) \boldsymbol{\sigma}_{1} \cdot \boldsymbol{m} \boldsymbol{\sigma}_{2} \cdot \boldsymbol{m} \\
& +(c-d) \boldsymbol{\sigma}_{1} \cdot \boldsymbol{l} \boldsymbol{\sigma}_{2} \cdot \boldsymbol{l}+i e\left(\boldsymbol{\sigma}_{1}+\boldsymbol{\sigma}_{2}\right) \cdot \boldsymbol{n}+g\left(\boldsymbol{\sigma}_{1} \cdot \boldsymbol{l} \boldsymbol{\sigma}_{2} \cdot \boldsymbol{m}+\boldsymbol{\sigma}_{1} \cdot \boldsymbol{m} \boldsymbol{\sigma}_{2} . \boldsymbol{l}\right)
\end{aligned}
$$




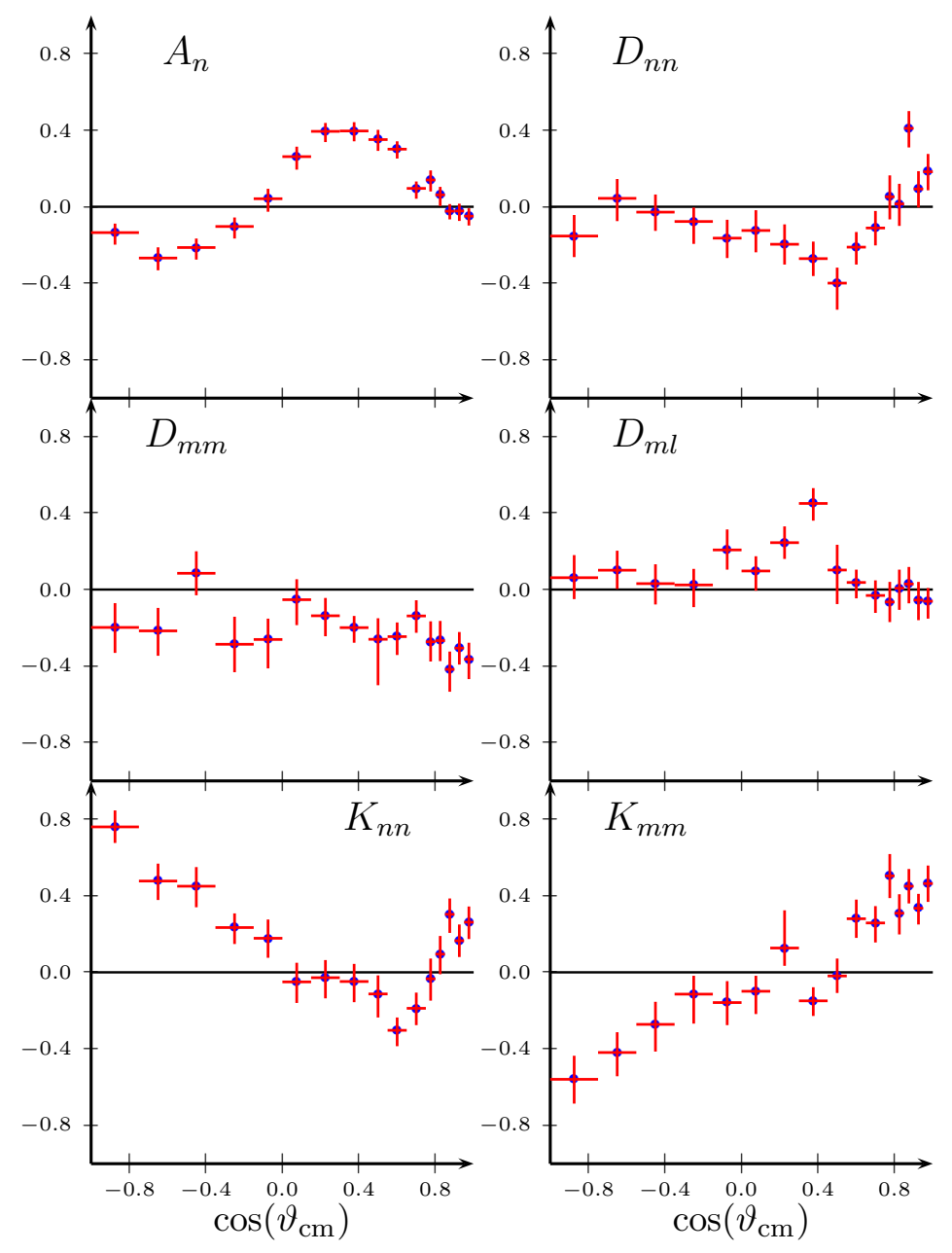

Figure 10: Some of the results of PS 185 [94] for the analysing power, depolarisation and transfer of polarisation of the reaction $\overline{\mathrm{p}} \mathrm{p} \rightarrow \bar{\Lambda} \Lambda$ at $p_{\mathrm{lab}}=1.637 \mathrm{GeV} / c$.

in terms of the unit vectors $\boldsymbol{l}, \boldsymbol{m}$ and $\boldsymbol{n}$ adapted to each particle, as per Eq. (2.2). If $I_{0}=\operatorname{Tr}\left[\mathcal{M M}^{\dagger}\right]$ is the differential cross section, the spin observables are defined as usual by

$$
\begin{aligned}
& C_{00 n 0} I_{0}=P_{n} I_{0}=\operatorname{Tr}\left[\boldsymbol{\sigma}_{1} . \boldsymbol{n} \mathcal{M M}^{\dagger}\right], \\
& C_{0 n 00} I_{0}=A_{n} I_{0}=\operatorname{Tr}\left[\mathcal{M} \boldsymbol{\sigma}_{2} . \boldsymbol{n} \mathcal{M}^{\dagger}\right], \\
& C_{00 i 0 j} I_{0}=D_{i j} I_{0}=\operatorname{Tr}\left[\boldsymbol{\sigma}_{2} \cdot \mathbf{\jmath} \mathcal{M} \boldsymbol{\sigma}_{2} . \boldsymbol{\imath} \mathcal{M}^{\dagger}\right] \text {, } \\
& C_{0 i j 0} I_{0}=K_{i j} I_{0}=\operatorname{Tr}\left[\boldsymbol{\sigma}_{1} \cdot \mathbf{j} \mathcal{M} \boldsymbol{\sigma}_{2} . \mathbf{\imath} \mathcal{M}^{\dagger}\right], \\
& C_{00 i j} I_{0}=C_{i j} I_{0}=\operatorname{Tr}\left[\boldsymbol{\sigma}_{1} . \imath \boldsymbol{\sigma}_{2} . \mathbf{\jmath} \mathcal{M M}^{\dagger}\right], \\
& C_{0 \alpha i j} I_{0}=C_{\alpha i j} I_{0}=\operatorname{Tr}\left[\boldsymbol{\sigma}_{1} . \imath \boldsymbol{\sigma}_{2} . \boldsymbol{\jmath} \mathcal{M} \boldsymbol{\sigma}_{2} . \boldsymbol{\alpha} \mathcal{M}^{\dagger}\right] \text {. }
\end{aligned}
$$


The spin observables (times $I_{0}$ ) which are accessible with a transversally polarised proton target are given by the quadratic relations

$$
\begin{aligned}
I_{0} & =|a|^{2}+|b|^{2}+|c|^{2}+|d|^{2}+|e|^{2}+|g|^{2}, & P_{n} I_{0} & =2 \Im \mathrm{m}\left(a e^{*}+d g^{*}\right), \\
C_{n n} I_{0} & =|a|^{2}-|b|^{2}-|c|^{2}+|d|^{2}+|e|^{2}+|g|^{2}, & A_{n} I_{0} & =2 \Im \mathrm{m}\left(a e^{*}-d g^{*}\right), \\
D_{n n} I_{0} & =|a|^{2}+|b|^{2}-|c|^{2}-|d|^{2}+|e|^{2}-|g|^{2}, & C_{m l} I_{0} & =2 \Re \mathrm{e}\left(a g^{*}-d e^{*}\right), \\
K_{n n} I_{0} & =|a|^{2}-|b|^{2}+|c|^{2}-|d|^{2}+|e|^{2}-|g|^{2}, & D_{m m} I_{0} & =2 \Re \mathrm{e}\left(a b^{*}+c d^{*}\right), \\
C_{m m} I_{0} & =2 \Re \mathrm{e}\left(a d^{*}+b c^{*}-g e^{*}\right), & D_{m l} I_{0} & =2 \Re \mathrm{e}\left(c g^{*}+b e^{*}\right), \\
C_{l l} I_{0} & =2 \Re \mathrm{e}\left(-a d^{*}+b c^{*}+g e^{*}\right), & K_{m m} I_{0} & =2 \Re \mathrm{e}\left(a c^{*}+b d^{*}\right), \\
C_{n l m} I_{0} & =2 \Im \mathrm{m}\left(g e^{*}-a^{*} d+b^{*} c\right), & K_{m l} I_{0} & =2 \Re \mathrm{e}\left(b g^{*}+c e^{*}\right), \\
C_{n m l} I_{0} & =2 \Im \mathrm{m}\left(g e^{*}-a^{*} d-b^{*} c\right), & C_{n m m} I_{0} & =2 \Im \mathrm{m}\left(d e^{*}-a g^{*}\right), \\
C_{m n l} I_{0} & =2 \Im \mathrm{m}\left(a b^{*}-c d^{*}\right), & C_{m l n} I_{0} & =2 \Im \mathrm{m}\left(a c^{*}-b d^{*}\right), \\
C_{m n m} I_{0} & =2 \Im \mathrm{m}\left(b e^{*}+c g^{*}\right), & C_{m m n} I_{0} & =2 \Im \mathrm{m}\left(c e^{*}+b g^{*}\right),
\end{aligned}
$$

supplemented by (see, e.g., [91])

$$
\begin{aligned}
C_{000 n} & =C_{00 n 0}, & C_{n n n} & =A_{n}, \\
C_{l m} & =C_{m l}, & C_{n l l} & =C_{n m m},
\end{aligned}
$$

the first identity meaning that $\bar{\Lambda}$ and $\Lambda$ have the same polarisation,

A purely algebraic $b \leftrightarrow c$ symmetry is observed, which, for instance, leaves $C_{n n}$ invariant and exchanges $D_{n n}$ and $K_{n n}$. The pairs $\left\{D_{n n}, C_{n n}\right\}$ and $\left\{K_{n n}, C_{n n}\right\}$ are, indeed, submitted to the same constraints, which will be studied in the next subsection. This symmetry corresponds to a single particle interchange: the observables for $\bar{p} p \rightarrow \Lambda \bar{\Lambda}$ are just a relabelling of these for $\overline{\mathrm{p}} \mathrm{p} \rightarrow \bar{\Lambda} \Lambda$. More permutation properties of (3.17) are discussed in [47].

\subsubsection{Empirical approach}

Richard and Elchikh $[27,28]$ have studied the inequalities relating pairs of $\bar{p} p \rightarrow \bar{\Lambda} \Lambda$ observables in a empirical but systematic way. The investigation has been extended to triples of observables [29,31]. The method simply consists of generating random, fictitious amplitudes $a, b$, etc. ( $a$ can be chosen real and positive), computing the observables (3.17), and plotting the results the one against the other. Once the contours revealed, it can be attempted to demonstrate rigorously the corresponding inequalities.

Whilst for pairs of observables, there are only three possibilities, for triples of observables, many more cases have been identified. Some of them have already been studied $[29,31,97]^{11}$. A more comprehensive survey is done below.

Pairs of observables For pairs of observables, either the full square $[-1,+1]^{2}$ is covered or a subdomain, which is the unit disk or a triangle, as shown in the examples given in Fig. 11: $P_{n}$ vs. $A_{n}$ (square), $A_{n}$ vs. $D_{m m}$ (disk) and $P_{n}$ vs. $C_{n n}$ (triangle). These plots correspond to 2000 random choices of amplitudes. To lighten the figure, some points inside the convex domain of the previous ones are omitted. The results are summarised in Table 4 for the sixteen observables listed in (3.17).

Note that the randomly generated amplitudes favours the centre of the plot, and doubt can be cast on whether the boundaries are actually reached. Instead, one can force the real or imaginary part of the amplitudes to be either 0 or \pm 1 , as in Fig. 12, where the border of the square, disk or triangle are clearly seen to be reached for special values of the observables.

\footnotetext{
${ }^{11}$ The "twisted cushion" shown in the above references turns out to be, by more careful investigation, a straight tetrahedron whose volume is slightly smaller.
} 

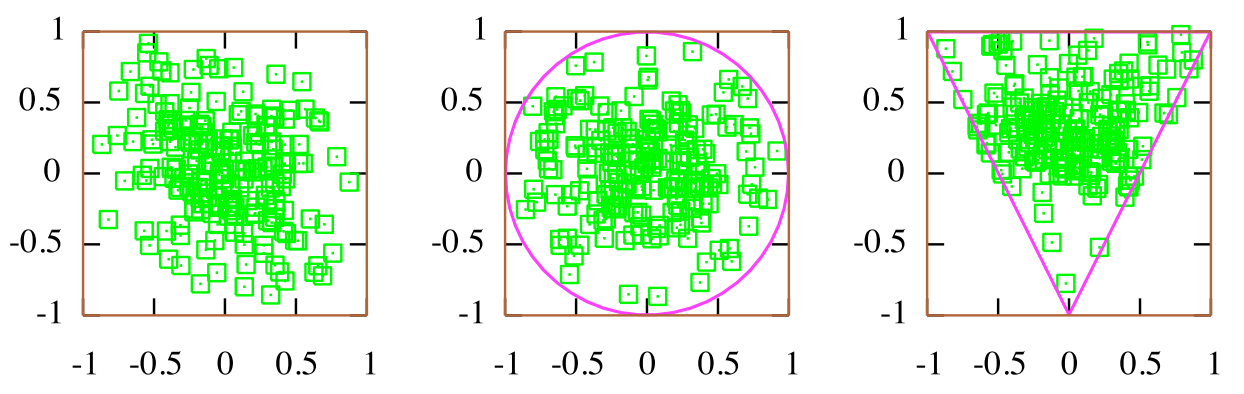

Figure 11: Random simulation of $P_{n}$ vs. $A_{n}$ (square), $A_{n}$ vs. $D_{m m}$ (disk) and $P_{n}$ vs. $C_{n n}$ (triangle)

Table 4: Domain allowed for pairs of observables: the entire square $(\square)$, the unit disk $(\bigcirc)$, the triangle $\left|2 \mathcal{O}_{1}\right| \leq \mathcal{O}_{2}+1(\nabla)$, or $\left|2 \mathcal{O}_{2}\right| \leq \mathcal{O}_{1}+1(\triangleleft)$, where $\mathcal{O}_{1}$ is horizontal and $\mathcal{O}_{2}$ vertical. The symbol $\otimes$ indicates that the pair of observables is constrained in the unit disk, but the corresponding operators do not anticommute.
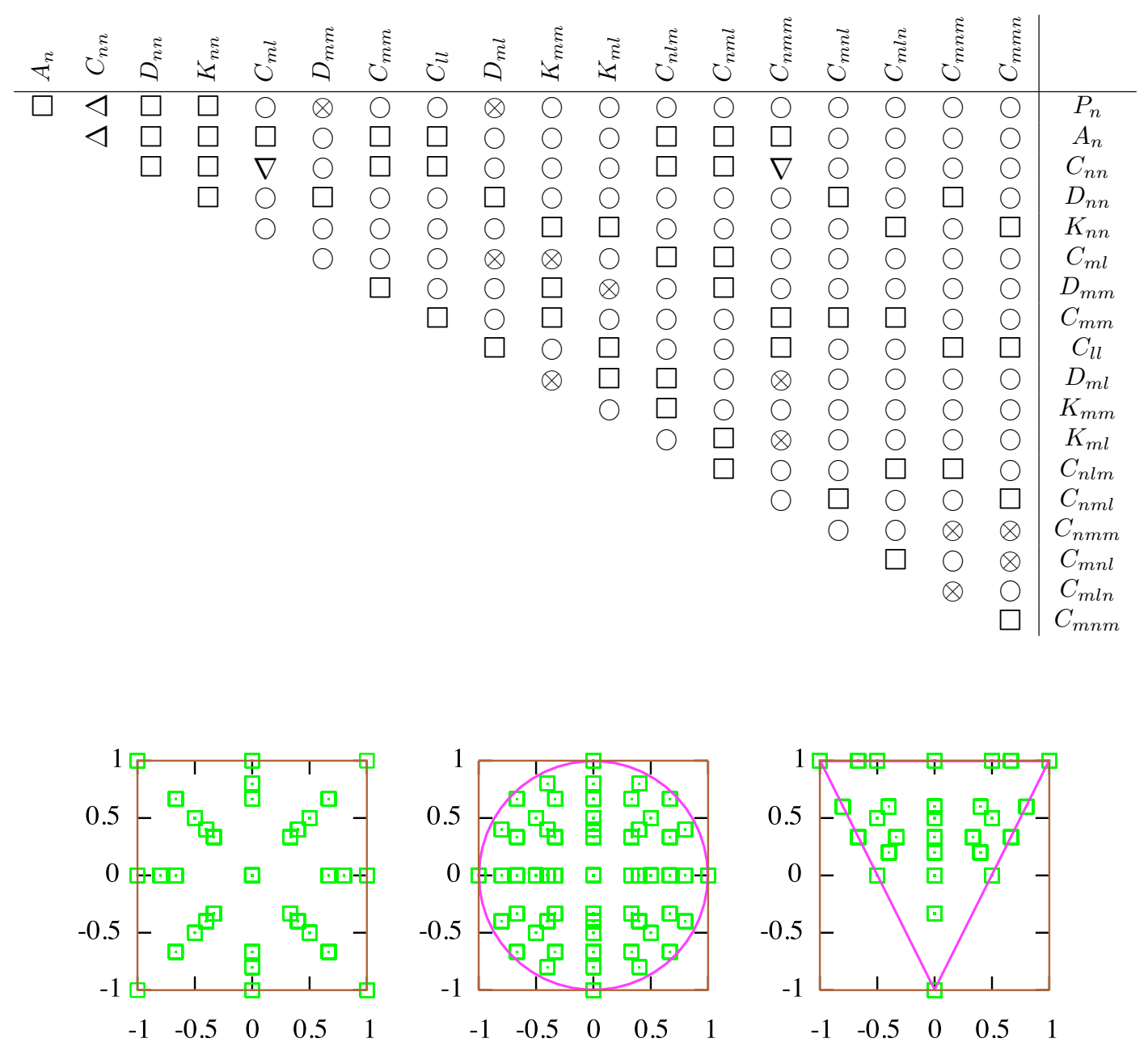

Figure 12: Same as Fig. 11, but each real or imaginary part amplitude is forced to be either 0 or \pm 1 . 
Triples of observables For three observables, there is a larger variety of situations. Some of them are direct consequences of the observations made for pairs, while other indicate new patterns that cannot be anticipated from the projections.

Figure 13 shows the boundary of the domains we have identified for the observables listed in (3.17): the unit sphere, a pyramid, an upside-down tent, a cone, a cylinder, the intersection of two orthogonal cylinders or a double cone which is slightly smaller than this intersection, a combination of the disk, square and triangle projections delimiting a volume similar to a "coffee filter", the intersection of three orthogonal cylinders (larger than the unit sphere!), a tetrahedron, the intersection of two cylinders and two planes, an octahedron, or figures deduced by mirror symmetry.

There are many cases where the three pairs are limited to the unit disk, and the three observables constrained inside the unit sphere, as for $\left\{P_{n}, D_{m m}, C_{l l}\right\}$, shown in Fig. 14.

There are a few cases, $\left\{A_{n}, D_{m m}, K_{m l}\right\},\left\{A_{n}, D_{m l}, K_{m m}\right\},\left\{C_{m l}, D_{m m}, D_{m l}\right\},\left\{C_{m l}, D_{m m}\right.$, $\left.K_{m l}\right\},\left\{C_{m l}, D_{m l}, K_{m m}\right\},\left\{C_{m l}, K_{m m}, K_{m l}\right\},\left\{D_{m m}, D_{m l}, C_{n m m}\right\}$, and $\left\{K_{m m}, K_{m l}, C_{n m m}\right\}$, where each projection is contained in the unit disk, but nevertheless, the domain is slightly larger than the unit sphere. The example of $\left\{A_{n}, D_{m m}, K_{m l}\right\}$ is shown in Fig. 15.

There are many examples where, in a triple, two pairs are constrained in the unit disk, while the projection of the third pair occupies the entire square. The domain is in general the intersection of the two cylinders. The case of $\left\{D_{m m}, P_{n}, A_{n}\right\}$ is displayed in Fig. 16. For $\left\{C_{m l}, C_{m m}, C_{l l}\right\}$, also shown in Fig. 16, a smaller inner domain has been identified: the cone of vertex $(-1,0,-1)$ and joining the ellipsis which is at the intersection of the two cylinders, and the symmetric cone.

The next category is the simple cylinder. An example is $\left\{A_{n}, C_{m l}, C_{m m}\right\}$, see Fig. 14: there is no constraint on $\left\{A_{n}, C_{m l}\right\}$ nor on $\left\{A_{n}, C_{m m}\right\}$, while $\left\{C_{m l}, C_{m m}\right\}$ is constrained in a disk.

As seen in Table 4, there are just a few cases of triangular constraint on pairs. Hence it is rather rare to find a triple with two triangles combined with a disk, to make a cone, as in the example of $\left\{P_{n}, C_{m l}, C_{n n}\right\}$, shown in Fig. 17. The two other cases are $\left\{P_{n}, C_{n m m}, C_{n n}\right\}$, and $\left\{C_{m l}, C_{n m m}\right.$, $\left.C_{n n}\right\}$.

Another type of cone, with square section, i.e., a pyramid, can be found with two pairs in a triangular constraint, and no constraint on the third pair, as for $\left\{P_{n}, A_{n}, C_{n n}\right\}$, shown in Fig. 17.

In the case of $\left\{P_{n}, A_{n}, D_{n n}\right\}$, there is no restriction on each pair, but the three observables are restricted inside a tetrahedron, which occupies only $1 / 3$ of the volume of the cube. A similar tetrahedron, but inverted, is obtained for $\left\{C_{n n}, C_{l l}, C_{m m}\right\}$, see Fig. 18.

Besides these tetrahedrons, the six triples of observables $\left\{P_{n}, D_{n n}, K_{n n}\right\},\left\{A_{n}, D_{n n}, K_{n n}\right\}$, $\left\{A_{n}, C_{m m}, C_{l l}\right\},\left\{A_{n}, C_{n l m}, C_{n m l}\right\},\left\{C_{m l}, C_{n l m}, C_{n m l}\right\}$, and $\left\{C_{m m}, C_{l l}, C_{n m m}\right\}$, also do not show any constraint on each pair, but do not occupy the entire cube. Their domain is an octahedron, as shown in Fig. 19, or an octahedron deduced by interchange of axes or parity.

The case of observables $\left\{P_{n}, C_{m m}, C_{n n}\right\}$ is remarkable: a pair has a disk constraint, another pair a triangular constraint, and the third one, no constraint. The result looks like a paper filter for coffee, see Fig. 19, left.

The case of $\left\{C_{m l}, C_{n l m}, C_{n n}\right\}$ is simpler, with a triangle and two squares seen in projection. The domain is the inverted tent seen in Fig. 20.

The triple $\left\{A_{n}, D_{m m}, C_{n n}\right\}$, shown in Fig. 20, has a boundary made by the intersection of two cylinders and a set of two planes (dihedral).

It should be stressed again that the figures shown in Fig. 13 do not exhaust all possibilities. If longitudinal polarisation is allowed, and if the antiproton beam is polarised, new observables can be reached. In particular, for any three out of the four observables $\left\{C_{m m}, C_{l l}, A_{m m}, A_{l l}\right\}$, i.e., final- and initial-spin correlation in the scattering plane, the domain corresponds to a new type of octahedron, shown in Fig. 21.

But the most important message is that, whilst the allowed domain of pairs is often the entire unit square, there is always a constraint for three observables, that limits the allowed domain to a volume smaller than the unit cube, in agreement with the reasoning of Sec. 2.6.3. 

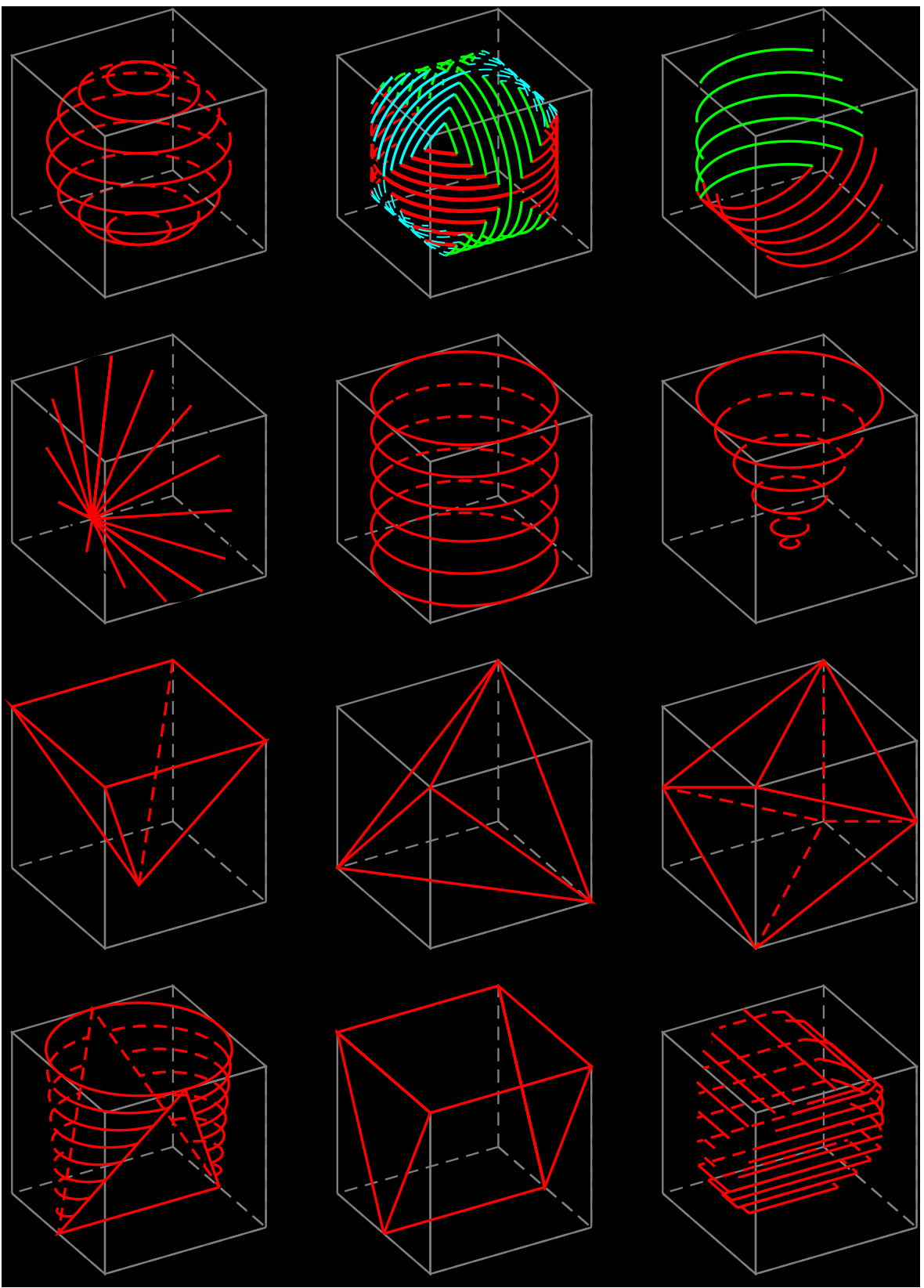

Figure 13: Some allowed domains encountered in simulating randomly three observables: the unit sphere (a), the intersection of three orthogonal cylinders of unit radius (b), the intersection of two cylinders (c), or a slightly smaller double cone (d), a cylinder (e), a cone (f), a pyramid (g), a tetrahedron (h), an octahedron (i), a "coffee filter" (j), an inverted tent (k), and the intersection of two cylinders and a dihedral (l). For clarity, part of the limiting surface is sometimes removed. Some figures transformed by parity with respect to the centre of the cube or by interchange of the axes are also obtained. 

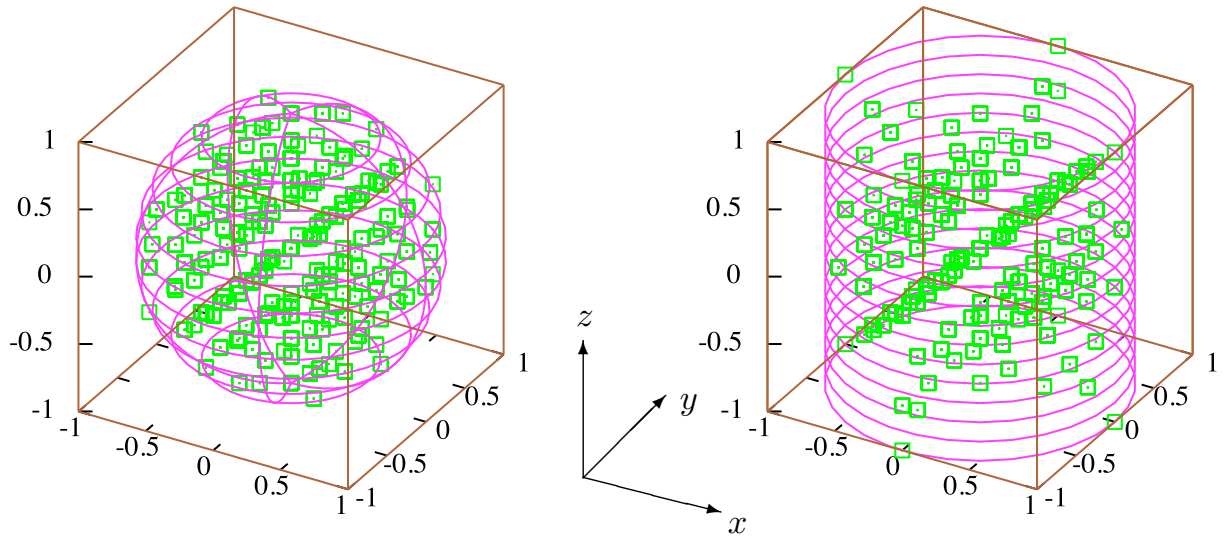

Figure 14: Simulation of the domain allowed for the observables $\left\{P_{n}, D_{m m}, C_{l l}\right\}$ (left) and $\left\{C_{m l}, C_{m m}, A_{n}\right\}$ (right), using amplitudes whose real and imaginary parts are chosen to be either 0 or \pm 1 .
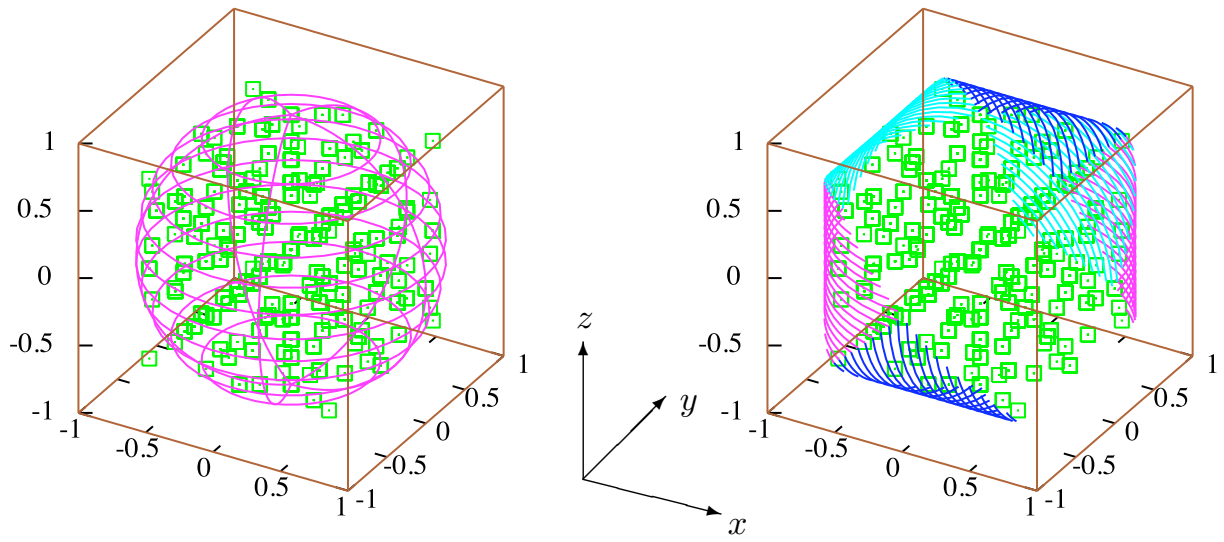

Figure 15: Fictitious observables $\left\{A_{n}, D_{m m}, K_{m l}\right\}$ obtained with amplitudes whose real and imaginary parts are chosen to be either 0 or \pm 1 , shown against the unit sphere (left) or the intersection of the three unit cylinders. 

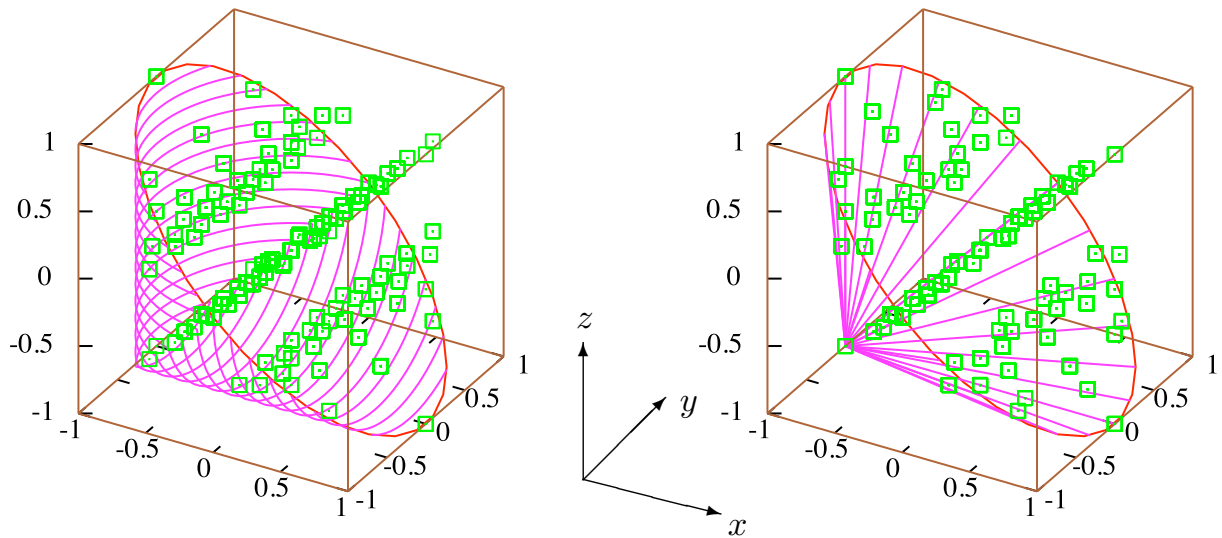

Figure 16: The domain for $\{x, y, z\}=\left\{P_{n}, D_{m m}, A_{n}\right\}$ (left) is inside the intersection of two cylinders, while the domain for $\left\{C_{m l}, C_{m m}, C_{l l}\right\}$ (right) is inside a slightly smaller double cone. For clarity, only the lower part of the boundary is shown..
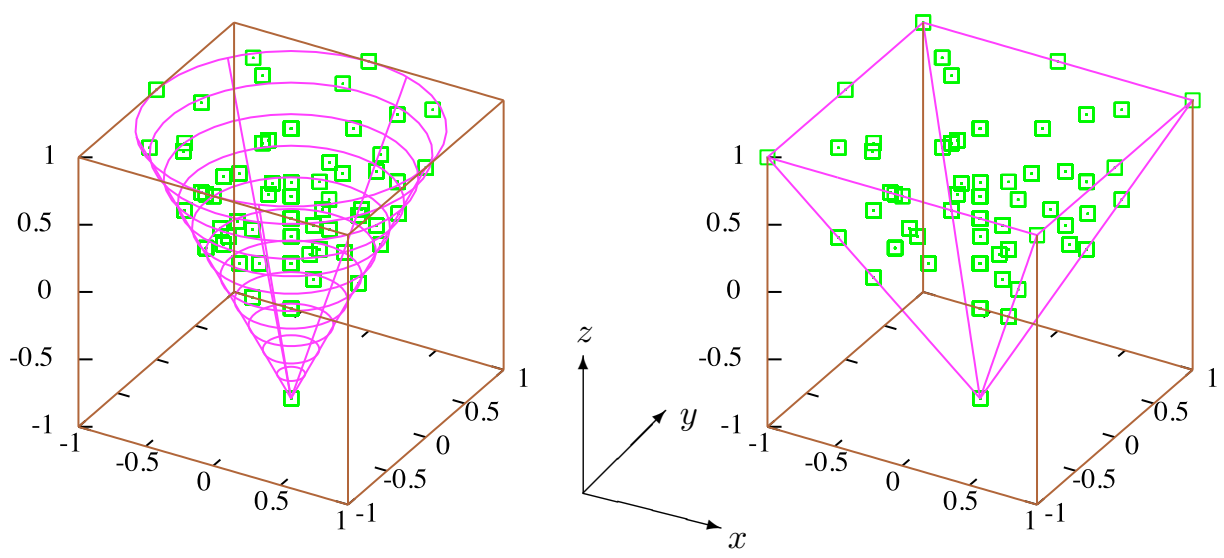

Figure 17: Domain for $\left\{P_{n}, C_{m l}, C_{n n}\right\}$ (left) and $\left\{P_{n}, A_{n}, C_{n n}\right\}$ (right). 

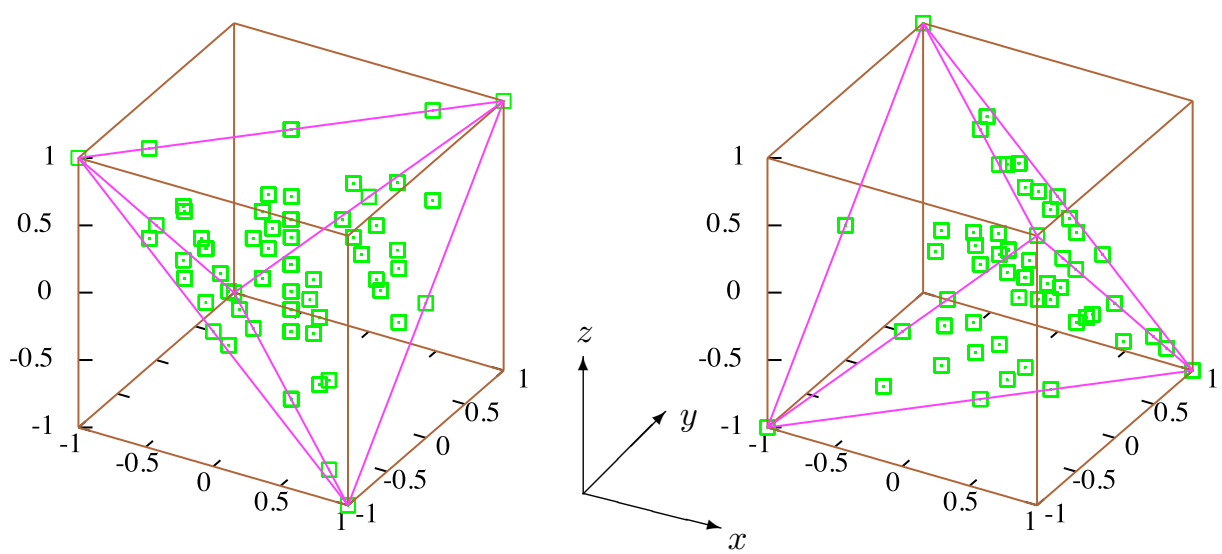

Figure 18: Domain for $\left\{P_{n}, A_{n}, D_{n n}\right\}$ (left) and $\left\{C_{n n}, C_{m m}, C_{l l}\right\}$ (right).
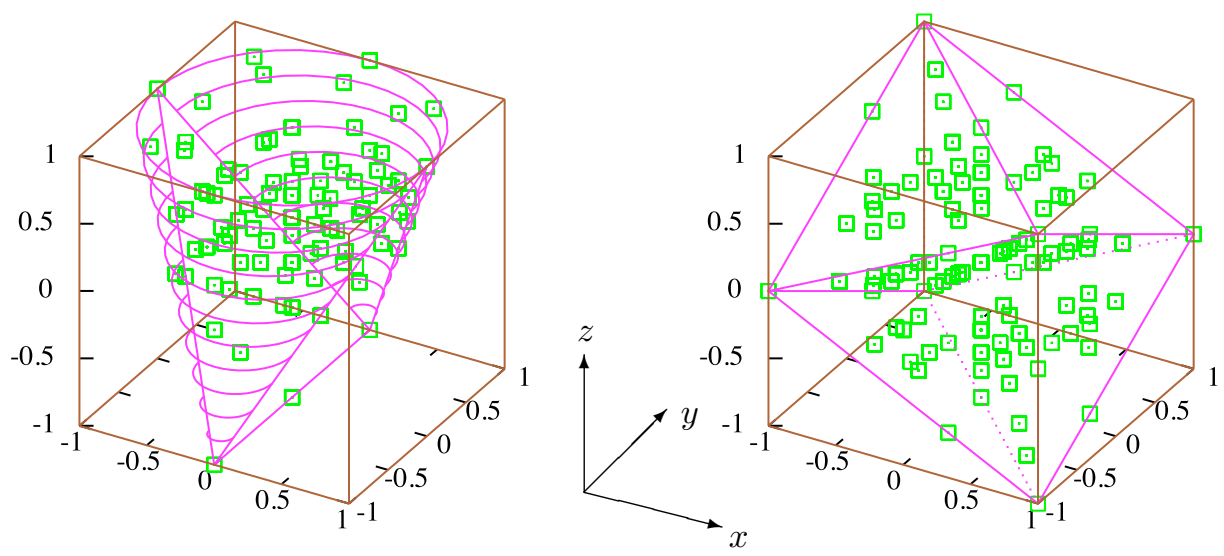

Figure 19: Observables $\left\{P_{n}, C_{m m}, C_{n n}\right\}$ (left) and $\left\{C_{m m}, C_{l l}, C_{n m m}\right\}$ (right). 

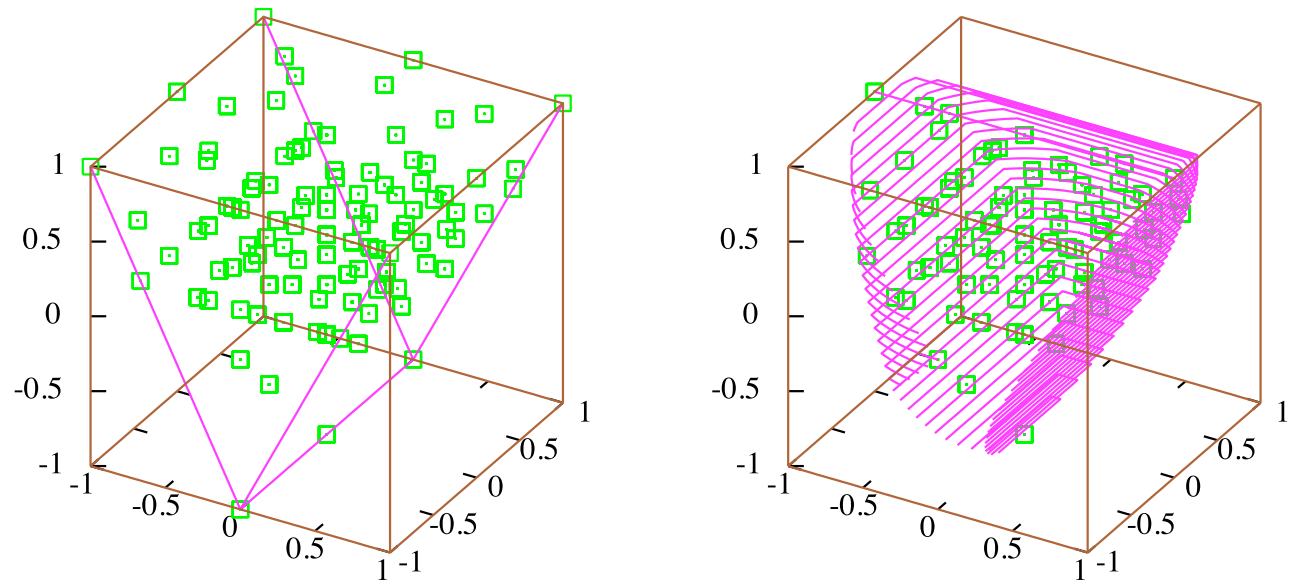

Figure 20: Simulation by random amplitudes of the observables $\left\{C_{m l}, C_{n l m}, C_{n n}\right\}$ (left) and $\left\{A_{n}, D_{m m}, C_{n n}\right\}$ (right).

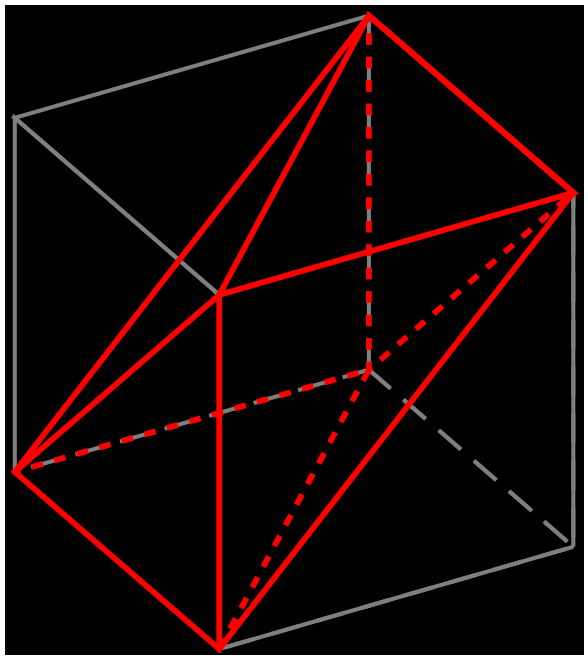

Figure 21: Any triple out of $\left\{C_{m m}, C_{l l}, A_{m m}, A_{l l}\right\}$ is constrained within an octahedron obtained by slicing off two opposite corners of the unit cube. 


\subsubsection{Understanding the observed domains}

Many of the inequalities detected by random simulations can be derived very simply. Others require somewhat the more sophisticated methods presented in Sec.2.6.

Anticommutation or commutation Many of the observed disk or sphere constraints are due to anticommuting operators, see Sec. 2.6.2. In particular, $\left(P_{n}, C_{i j}\right)$ is in a disk for $i=l$ or $m$ and any $j \in\{l, m, n\}$, since the corresponding operators $\left\langle I \otimes I \otimes \sigma_{n} \otimes I\right\rangle$ and $\left\langle I \otimes I \otimes \sigma_{i} \otimes \sigma_{j}\right\rangle$ anticommute. Note that for $j=n$ the domain is restricted to the diameter $C_{i n}=0$ of the disk, due to parity conservation. Similarly $\left(P_{n}, C_{i j}, K_{h i^{\prime}}\right)$ is in a sphere if $i \neq n, i^{\prime} \neq i$ and $i^{\prime} \neq n$, for any $h$ and $j$. Other examples are $K_{n n}^{2}+C_{l l}^{2}+O_{n l m}^{2} \leq 1$, and $D_{n n}^{2}+C_{l l}^{2}+O_{n l m}^{2} \leq 1$.

When $\mathcal{O}$ and $\mathcal{O}^{\prime}$ commute, the domain of $\left(\langle\mathcal{O}\rangle,\left\langle\mathcal{O}^{\prime}\right\rangle\right)$ may reach a corner of the square $[-1,+1]^{2}$. This is the case, e.g., for $\left(P_{n}, C_{n n}\right)$ (triangle) or $\left(D_{m m}, C_{n m l}\right)$ (whole square). In the cases indicated by a $\oplus$ in Table 2, however, the domain is a disk as if $\mathcal{O}$ and $\mathcal{O}^{\prime}$ were anticommuting. The reason is that some symmetry imposes $\mathcal{O}^{\prime}=\mathcal{O}^{\prime \prime}$ where $\mathcal{O}^{\prime \prime}$ anticommutes with $\mathcal{O}$. For instance $\left(D_{m l}, C_{m l}\right)$ is a commuting pair, but charge conjugation imposes $C_{m l}=C_{l m}$ and $C_{l m}$ anticommutes with $D_{m l}$.

Final-state density matrix Inequalities for the $\bar{p} p \rightarrow \bar{\Lambda} \Lambda$ reaction relevant to a polarised target have been obtained in [30] using the positivity of the final state $4 \times 4$ density matrix for a given target polarisation $\boldsymbol{S}_{\mathrm{p}}$ (see also [27,28]),

$$
\rho_{\bar{\Lambda} \Lambda}\left(\boldsymbol{S}_{\mathrm{p}}\right)=\frac{1}{\mathcal{I}\left(\boldsymbol{S}_{\mathrm{p}}\right)} \mathcal{M} \rho_{\overline{\mathrm{p}} \mathrm{p}} \mathcal{M}^{\dagger}, \quad \text { with } \quad \rho_{\overline{\mathrm{p} p}}=\sum_{\lambda} S_{\mathrm{p}}^{\lambda} \frac{\mathbb{1} \otimes \sigma^{\lambda}}{4} .
$$

$\mathcal{I}\left(\boldsymbol{S}_{\mathrm{p}}\right)=\operatorname{Tr}\left\{\mathcal{M} \rho_{\overline{\mathrm{p}} \mathrm{p}} \mathcal{M}^{\dagger}\right\}$ is the cross section when only the target is polarised. In terms of the Cartesian reaction parameter,

$$
\rho_{\overline{\Lambda \Lambda}}\left(\boldsymbol{S}_{\mathrm{p}}\right)=\frac{\mathcal{I}(0)}{4 \mathcal{I}\left(\boldsymbol{S}_{\mathrm{p}}\right)} \sum_{\lambda, \mu, \nu=0, l, m, n} S_{\mathrm{p}}^{\lambda} C_{\lambda \mu \nu}\left(\sigma^{\mu} \otimes \sigma^{\nu}\right) .
$$

For transverse $S_{\mathrm{p}}$, one can also write

$$
\rho_{\bar{\Lambda} \Lambda}\left(\boldsymbol{S}_{\mathrm{p}}\right)=\frac{\mathcal{I}(0)}{4 \mathcal{I}\left(\boldsymbol{S}_{\mathrm{p}}\right)}\left(\Gamma_{0}+\left|\boldsymbol{S}_{\mathrm{p}}\right| \cos \phi \Gamma_{n}+\left|\boldsymbol{S}_{\mathrm{p}}\right| \sin \phi \Gamma_{m}\right),
$$

where $\phi$ is the azimuthal angle of $\boldsymbol{S}_{\mathrm{p}}$ in the $\left\{\boldsymbol{l}_{\mathrm{p}}, \boldsymbol{m}_{\mathrm{p}}, \boldsymbol{n}\right\}$ frame. For instance, in the helicity basis,

$$
\Gamma_{0}=\left(\begin{array}{cccc}
1-C_{l l} & -C_{m l}-i P_{n} & -C_{m l}-i P_{n} & -C_{n n}-C_{m m} \\
-C_{m l}+i P_{n} & 1+C_{l l} & C_{n n}-C_{m m} & C_{m l}-i P_{n} \\
-C_{m l}+i P_{n} & C_{n n}-C_{m m} & 1+C_{l l} & C_{m l}-i P_{n} \\
-C_{n n}-C_{m m} & C_{m l}+i P_{n} & C_{m l}+i P_{n} & 1-C_{l l}
\end{array}\right) .
$$

The quadratic inequalities of the type $\rho_{11} \rho_{22} \geq\left|\rho_{12}\right|^{2}$, applied to the above $\Gamma_{0}$ gives for instance

$$
\begin{aligned}
C_{m l}^{2}+P_{n}^{2}+C_{l l}^{2} & \leq 1, \\
\left(C_{n n}-C_{m m}\right)^{2} & \leq\left(1+C_{l l}\right)^{2} .
\end{aligned}
$$

Similarly, from

$$
\Gamma_{n}=\left(\begin{array}{cccc}
A_{n}+O_{n m m} & -O_{n l m}-i D_{n n} & -O_{n m l}-i K_{n n} & -A_{n}-O_{n m m} \\
-O_{n l m}+i D_{n n} & A_{n}-O_{n m m} & A_{n}-O_{n m m} & O_{n m l}-i K_{n n} \\
-O_{n m l}+i K_{n n} & A_{n}-O_{n m m} & A_{n}-O_{n m m} & O_{n l m}-i D_{n n} \\
-A_{n}-O_{n m m} & O_{n m l}+i K_{n n} & O_{n l m}+i D_{n n} & A_{n}+O_{n m m}
\end{array}\right)
$$

corresponding to the proton spin along $\boldsymbol{n}$, one can derive

$$
\begin{gathered}
O_{n m m}^{2}+O_{n l m}^{2}+C_{m l}^{2}+P_{n}^{2}+D_{n n}^{2}+C_{l l}^{2} \leq 1+A_{n}^{2}, \\
K_{n n}^{2}+C_{l l}^{2}+O_{n l m}^{2} \leq 1 .
\end{gathered}
$$


Cross section matrix (CSM) The positivity of $\rho_{\overline{\Lambda \Lambda}}\left(S_{\mathrm{p}}\right)$ does not give all possible inequalities for three polarised particles. In particular, it misses the positivity constraints required by "entangled states in the $t$ - or $u$-channel". The CSM gives the complete set of inequalities. Let us apply it here, following the method of [31], the CSM of $\bar{p} p \rightarrow \bar{\Lambda} \Lambda$ can be defined as

$$
\left\langle s_{\overline{\mathrm{p}}}^{\prime}, s_{\mathrm{p}}^{\prime}, s_{\bar{\Lambda}}^{\prime}, s_{\Lambda}^{\prime}|\mathcal{R}| s_{\overline{\mathrm{p}}}, s_{\mathrm{p}}, s_{\bar{\Lambda}}, s\right\rangle=\left\langle s_{\overline{\mathrm{p}}}^{\prime}, s_{\mathrm{p}}^{\prime}\left|\mathcal{M}^{\dagger}\right| s_{\bar{\Lambda}}^{\prime}, s_{\Lambda}^{\prime}\right\rangle\left\langle s_{\bar{\Lambda}}, s_{\Lambda}|\mathcal{M}| s_{\overline{\mathrm{p}}}, s_{\mathrm{p}}\right\rangle .
$$

By construction $R$ is semi-positive definite and of rank 1 . $\mathcal{R}$ is given terms of the correlation parameters, as per Eq. (2.73).

Let us use the transversity basis where $\sigma_{y}=\sigma_{3}$ is diagonal, and put the $\left|s_{\overline{\mathrm{p}}}, s_{\mathrm{p}}, s_{\bar{\Lambda}} s_{\Lambda}\right\rangle$ states in the following order: $|++++\rangle,|+++-\rangle,|++-+\rangle,|++--\rangle$, etc., like numbers in base 2 , but with 0 and 1 being replaced by + and - . Due to parity conservation, $\mathcal{M}$ is zero when there is one + sign and three - signs or vice-versa. Therefore $\mathcal{R}$ has the block-diagonal form $\mathcal{R}^{+} \oplus \mathbf{0}$, where $R^{+}$ is the $8 \times 8$ upper-left submatrix of $\mathcal{R}$, and $\mathbf{0}$ the null matrix. One can also consider Eq. (2.99). Using $\Pi_{F}=i \xi_{F} \sigma_{F}^{3}$ and $\xi_{F} \xi_{\bar{F}}=-1$ for each fermion $F$, it becomes

$$
\sigma_{3} \otimes \sigma_{3} \otimes \sigma_{3} \otimes \sigma_{3} \mathcal{R}=\mathcal{R} \sigma_{3} \otimes \sigma_{3} \otimes \sigma_{3} \otimes \sigma_{3}=\mathcal{R},
$$

which is the Bohr identity [63]. From this one concludes that the $(\lambda, \mu \mid \nu, \tau)$ 's are invariant under the substitutions: $0 \leftrightarrow 3,1 \rightarrow i 2,2 \rightarrow-i 1$ for $\lambda$ and $\mu$ and $0 \leftrightarrow 3,1 \rightarrow-i 2,2 \rightarrow i 1$ for $\nu$ and $\tau$, where, for instance $(\lambda, \mu \mid \nu, i \tau) \equiv i(\lambda, \mu \mid \nu, \tau)$.

The explicit form of $\mathcal{R}^{+}$in terms of the $(\lambda \mu \mid \nu \tau)$ 's will be needed when both beam and target will be polarised. We shall not write it here. Instead we consider the reduced CSM, $\mathcal{R}_{3}=\operatorname{Tr}_{\overline{\mathrm{p}}} \mathcal{R}$ describing the 3-particle observables between $\mathrm{p}, \Lambda$ and $\bar{\Lambda}$. It can be decomposed in the blockdiagonal form $\mathcal{R}_{3}=\mathcal{R}_{3}^{+} \oplus \mathcal{R}_{3}^{-}$where $\mathcal{R}_{3}^{ \pm}=\operatorname{Tr}_{\overline{\mathrm{p}}} \mathcal{R}\left(\mathbb{1} \pm \sigma_{3}\right)_{\overline{\mathrm{p}}} / 2$ comes from antiprotons of + or transversity. $\mathcal{R}_{3}^{+}$and $\mathcal{R}_{3}^{-}$are the $4 \times 4$ upper-left and lower-right submatrices of $\mathcal{R}_{3}$. Table 5 gives the matrix elements of $\mathcal{R}_{3}^{+}$, normalised to $\operatorname{Tr} R_{3}^{+}=\operatorname{Tr} \mathbb{1}$.

Table 5: Submatrix $\mathcal{R}_{3}^{+}$of the Cross Section Matrix of $\overline{\mathrm{p}}+\mathrm{p} \uparrow \rightarrow \bar{\Lambda} \uparrow+\Lambda \uparrow$.

\begin{tabular}{c|cccc} 
& +++ & +-- & -+- & --+ \\
\hline+++ & $(0+3 \mid 0+3,0+3)$ & $(0+3 \mid 1+i 2,1+i 2)$ & $(1-i 2 \mid 0+3,1+i 2)$ & $(1-i 2 \mid 1+i 2,0+3)$ \\
+-- & $(0+3 \mid 1-i 2,1-i 2)$ & $(0+3 \mid 0-3,0-3)$ & $(1-i 2 \mid 1-i 2,0-3)$ & $(1-i 2 \mid 0-3,1-i 2)$ \\
-+- & $(1+i 2 \mid 0+3,1-i 2)$ & $(1+i 2 \mid 1+i 2,0-3)$ & $(0-3 \mid 0+3,0-3)$ & $(0-3 \mid 1+i 2,1-i 2)$ \\
--+ & $(1+i 2 \mid 1-i 2,0+3)$ & $(1+i 2 \mid 0-3,1+i 2)$ & $(0-3 \mid 1-i 2,1+i 2)$ & $(0-3 \mid 0-3,0+3)$
\end{tabular}

In this table, a compact notation is adopted. For instance

$$
\begin{aligned}
& (0+3 \mid 1-i 2,1+i 2)=\left\langle\left(\sigma_{\mathrm{p}}^{0}+\sigma_{\mathrm{p}}^{3}\right) \otimes\left(\sigma_{\Lambda}^{1}-i \sigma_{\Lambda}^{2}\right) \otimes\left(\sigma_{\Lambda}^{1}+i \sigma_{\Lambda}^{2}\right)\right\rangle= \\
& (00 \mid 11)+(03 \mid 11)-i(00 \mid 21)-i(03 \mid 21)+i(00 \mid 12)+i(03 \mid 12)+(00 \mid 22)+(03 \mid 22) .
\end{aligned}
$$

$\mathcal{R}_{3}^{-}$is obtained from $\mathcal{R}_{3}^{+}$by changing the sign before the last digit in each box of Table 5 . It can be also obtained by replacing each box by its symmetrical one about the centre of the matrix, then changing $\pm z$ into $\mp z$ and $\pm i 2$ into $\mp i 2$ everywhere. Both submatrices are of rank one, since each is associated to one spin state of the antiproton. Therefore all their $2 \times 2$ subdeterminants are zero. This gives a number of quadratic equations. A complete set of inequalities can - in principle - be derived from these relations and from the positivity of the diagonal elements. In particular, Eqs. (3.23-3.25) are recovered, although less quickly than using the helicity basis.

If the target is unpolarised, the CSM is further reduced to a $4 \times 4$ matrix $\mathcal{R}_{2}$, which after transposition and normalisation becomes $\rho_{\bar{\Lambda} \Lambda}(0)=\Gamma_{0} / 4$ (see Eqs.3.21-3.22). Let us rewrite $\Gamma_{0}$ in the transversity basis:

$$
\Gamma_{0}=\left(\begin{array}{cccc}
1+C_{n n}+2 P_{n} & 0 & 0 & C_{l l}-C_{m m}-2 i C_{l m} \\
0 & 1-C_{n n} & C_{l l}+C_{m m} & 0 \\
0 & C_{l l}+C_{m m} & 1-C_{n n} & 0 \\
C_{l l}-C_{m m}+2 i C_{l m} & 0 & 0 & 1+C_{n n}-2 P_{n}
\end{array}\right)
$$


where charge conjugation $C_{l m}=C_{m l}, P_{n}(\Lambda)=P_{n}(\bar{\Lambda})$ has been taken into account. As expected from the symmetry about the scattering plane, $\rho_{\bar{\Lambda} \Lambda}$ is block-diagonal in two rank-2 submatrices. This greatly simplifies the derivation of the positivity. One obtains the minimal complete set of constraints

$$
\begin{aligned}
\left(1+C_{n n}\right)^{2} & \geq 4 P_{n}^{2}+\left(C_{l l}-C_{m m}\right)^{2}+4 C_{l m}^{2}, \\
\left(1-C_{n n}\right)^{2} & \geq\left(C_{l l}+C_{m m}\right)^{2}, \\
\left|C_{n n}\right| & \leq 1,
\end{aligned}
$$

which, in particular, recovers Eqs.(3.23,3.25).

Projection on a subset of observables. Most often, only a subset of observables is measured and it is useful to draw the allowed domain for this subset. If, for instance $P_{n}$ and $C_{l m}$ are not measured, the answer is immediate, since the planes $P_{n}=0$ and $C_{l m}=0$ are symmetry planes for $\mathcal{D}$ : the allowed domain $\mathcal{D}_{3}\left\{C_{l l}, C_{m m}, C_{n n}\right\}$ is obtained by setting $P_{n}=C_{l m}=0$ in Eqs. (3.30), which gives

$$
\left|1+C_{n n}\right| \geq\left|C_{l l}-C_{m m}\right|, \quad\left|1-C_{n n}\right| \geq\left|C_{l l}+C_{m m}\right|, \quad 1 \geq\left|C_{n n}\right| .
$$

This is the tetrahedron of vertices $(-1,-1,-1),(-1,+1,+1),(+1,-1,+1),(+1,+1,-1)$ seen in Fig.18 (right).

Let us now consider the case where at least one of the non-measured variables does not correspond to a symmetry plane of $\mathcal{D}$. For illustration we assume that $C_{l m}$ and $C_{l l}$ are not measured and we look for $\mathcal{D}_{3}\left\{P_{n}, C_{m m}, C_{n n}\right\}$. First of all, since $C_{l m}=0$ is a symmetry plane of $\mathcal{D}$, one can immediately set $C_{l m}=0$ in (3.30). The projection on the non-symmetry plane $C_{l l}=0$ may be found with the two following different methods:

The apparent contour method. Let us rewrite the minimal complete set of constraints (3.30), with $C_{l m}=0$, as

$$
\begin{aligned}
f(v, x, y, z) & =(1+z)^{2}-(x-y)^{2}-4 v^{2} \geq 0 \\
g(x, y, z) & =(1-z)^{2}-(x+y)^{2} \geq 0 \\
h(z) & =1-z^{2} \geq 0
\end{aligned}
$$

where $v=P_{n}, x=C_{l l}, y=C_{m m}, z=C_{n n}$. The projection $\mathcal{D}_{3}$ of $\mathcal{D}_{4}$ defined by (3.32) on the plane $x=0$ can be obtained from the following rule:

The boundary or apparent contour of $\mathcal{D}_{3}$ is the projection of a curve $\mathcal{H}$ lying on the boundary of $\mathcal{D}_{4}$ that we call horizon. A systematic method to find the horizon is the following:

- If $\mathcal{D}_{4}$ were defined by only one inequality, say $f \geq 0$, a point of the horizon would satisfy

$$
f=0, \quad f_{x}^{\prime}=0, \quad f_{x}^{\prime \prime} \leq 0 .
$$

¿From the two equalities one would eliminate $x$ and obtain the wanted equation in $v, y, z$ for the boundary. The inequality $f_{x}^{\prime \prime} \leq 0$ is the requirement that the projection line does not go inside $\mathcal{D}_{4}$ but is externally tangent to it.

- Actually, in our example, $\mathcal{D}$ is the intersection of 3 domains $\mathcal{D}_{f}, \mathcal{D}_{g}, \mathcal{D}_{h}$ respectively defined by $f \geq 0, g \geq 0, h \geq 0$. The projection of $\mathcal{D}$ is generally smaller than the intersection of the projections of $\mathcal{D}_{f}, \mathcal{D}_{g}$ and $\mathcal{D}_{h}$. A point of $\mathcal{H}$ can in fact be

A) a point of the horizon $\mathcal{H}_{f}$ of $\mathcal{D}_{f}$, while belonging to $\mathcal{D}_{g}$ and $\mathcal{D}_{h}$ :

$$
f=0, \quad f_{x}^{\prime}=0, \quad f_{x}^{\prime \prime} \leq 0, \quad g \geq 0, \quad h \geq 0,
$$

or idem with permutation of the roles of $f, g$ and $h$. 
B) a point common to the boundaries of $\mathcal{D}_{f}$ and $\mathcal{D}_{g}$ and belonging to $\mathcal{D}_{h}$ :

$$
f=g=0, \quad f_{x}^{\prime} g_{x}^{\prime} \leq 0, \quad h \geq 0
$$

or idem with permutation of the roles of $f, g$ and $h$. The first inequality of (3.35) is the requirement that the projection line does not go inside $\mathcal{D}_{f} \cap \mathcal{D}_{g}$.

Let us apply these principles to (3.32).

a) $f=f_{x}^{\prime}=0, f_{x}^{\prime \prime} \leq 0, g \geq 0, h \geq 0$ gives the two triangles

$$
|z| \leq 1, \quad 2|y| \leq 1-z, \quad 2|v| \pm 1+z,
$$

b) $g=g_{x}^{\prime}=0, g_{x}^{\prime \prime} \leq 0, f \geq 0, h \geq 0$ gives the disk

$$
z=1, \quad v^{2}+y^{2} \leq 1 \quad(h=0),
$$

c) $h=h_{x}^{\prime}=0, h_{x}^{\prime \prime} \leq 0, f \geq 0, g \geq 0$ gives the same disk, plus the edge

$$
z=-1, v=0, \quad|y| \leq 1 \quad(f=0),
$$

d) $g=h=0, g_{x}^{\prime} h_{x}^{\prime} \leq 0, f \geq 0$ gives the disk again, plus the end points $A$ and $B$ of the edge,

$$
z=-1, v=0, \quad y= \pm 1 \quad(f=0),
$$

e) $f=h=0, f_{x}^{\prime} h_{x}^{\prime} \leq 0, g \geq 0$ gives the edge again plus the disk perimeter.

f) $f=g=0, f_{x}^{\prime} g_{x}^{\prime} \leq 0, h \geq 0$ gives two pieces of cones:

$$
|z| \leq 1, \quad \pm 2 y=1-z+\sqrt{(1+z)^{2}-4 v^{2}},
$$

$\mathcal{D}_{3}$ is then made of the convex completion of two cones, within the cube $[-1,+1]^{\otimes 3}$. The cones, of equations $(1-z \pm 2 y)^{2}+4 v^{2}=(1+z)^{2}$, have tips $(0, \mp 1,-1)$ and the disk (b) as a common basis. The walls looks like a paper coffee filter, see Fig. 19, left. The interior of the domain is given by

$$
|z| \leq 1, \quad 2|v| \leq 1+z, \quad|2 y|+z+\sqrt{(1+z)^{2}-4 v^{2}} \leq 1 .
$$

A section by a plane $z=$ constant is a stadium.

The method of the reciprocal polar transform [40]. Let us now try the second method outlined in Sec. 2.6.4. First of all, we recall that due to parity and charge conjugation the effectively allowed domain $\mathcal{D}_{\text {eff }}$ for the parameters $C_{\lambda \mu \nu \tau}$ is not the full domain of positivity $\mathcal{D}$ but the intersection of $\mathcal{D}$ with a hyperplane $H$. Then the vector $C_{\perp}$ defined in Sec. 2.6.4 has redundant coordinates, for instance $C_{00 n 0}=P_{n}$ and $C_{000 n}$, which are equal. Nevertheless, both coefficients contribute to the scalar product $\boldsymbol{C}_{\perp} \cdot \boldsymbol{C}_{\perp}^{\prime}$ in (2.84), so that their contributions add to $2 P_{n} P_{n}^{\prime}$. An equivalent statement is that the corresponding Euclidean coordinate in $H$ is : $\hat{P}_{n}=\left(C_{00 n 0}+C_{000 n}\right) / \sqrt{2}=P_{n} \sqrt{2}$. One must keep this fact in mind when applying the reciprocal polar transform.

We consider the intersection $\mathcal{I}_{3}\left\{P_{n}, C_{m m}, C_{n n}\right\}$ of $\mathcal{D}_{\text {eff }}$ with the hyperplane where all $C_{\lambda \mu \nu \tau}$ are vanishing except for $C_{0000} \equiv 1, C_{00 n 0}=C_{000 n} \equiv P_{n}, C_{00 m m} \equiv C_{m m}$ and $C_{00 n n} \equiv C_{n n}$. Using the notations of (3.32) it is equivalent to the intersection $\mathcal{I}_{3}$ of $\mathcal{D}_{4}$ with the hyperplane $x=0$ (up to the rescaling $\hat{P}_{n}=\hat{v}=v \sqrt{2}$ ). From (3.32), $\mathcal{I}_{3}$ is given by

$$
\begin{aligned}
f(v, 0, y, z) & =(1+z)^{2}-y^{2}-4 v^{2} \geq 0, \\
g(0, y, z) & =(1-z)^{2}-y^{2} \geq 0, \\
h(z) & =1-z^{2} \geq 0 .
\end{aligned}
$$


The first and last equations define the interior of a cone whose tip is the centre of the bottom face of the $[-1,1]^{\otimes 3}$ cube and whose intersection with the top face plane $(z=1)$ is the ellipse $v^{2}+(y / 2)^{2}=$ 1. Due to the second equation, this cone is amputated by a "roof" made of the two planes $z=1 \pm y$.

According to Sec.2.6.4 the domain $\mathcal{D}_{3}$ we are looking for is the set of points $C_{\perp}=(v, y, z)$ which satisfy

$$
C_{\perp} \cdot C_{\perp}^{\prime} \geq-1
$$

for any $C_{\perp}^{\prime} \in \mathcal{I}_{3}$, the scalar product in $\mathcal{D}_{3}$ being given by

$$
\boldsymbol{C}_{\perp} \cdot \boldsymbol{C}_{\perp}^{\prime}=2 v \cdot v^{\prime}+y \cdot y^{\prime}+z \cdot z^{\prime} \quad \text { or } \quad \hat{v} \cdot \hat{v}^{\prime}+y \cdot y^{\prime}+z \cdot z^{\prime} .
$$

The boundary $\partial \mathcal{D}_{3}$ is the reciprocal polar transform, of power -1 , of the boundary $\partial \mathcal{I}_{3}$ of $\mathcal{I}_{3}$.

Instead of using (3.44) we may keep the usual form $\boldsymbol{C}_{\perp} \cdot \boldsymbol{C}_{\perp}^{\prime}=v \cdot v^{\prime}+y \cdot y^{\prime}+z \cdot z^{\prime}$ but dilate $\mathcal{I}_{3}$ alone by a factor 2 in the $v$ dimension. It has the advantage of transforming the cone of $\mathcal{I}_{3}$ into a cone of revolution. Then $\partial \mathcal{D}_{3}$ is the reciprocal polar transform of $\partial \mathcal{I}_{3}^{\prime}$, the boundary of the dilated $\mathcal{I}_{3}$, with the usual scalar product. Using the correspondences listed in (2.20), one recovers the various pieces of the "coffee filter":

$$
\begin{array}{clc}
\text { pieces of } \partial \mathcal{I}_{3}^{\prime}: & & \text { pieces of } \partial \mathcal{D}_{3}: \\
\text { cone tip t } & \rightarrow & \text { disk-face CFDG } \\
\text { trihedron-like tips c and d } & \rightarrow & \text { triangular faces ABC and ABD } \\
\text { top edge cd } & \rightarrow & \text { bottom edge AB } \\
\text { edges cfd and cgd (parabolas) } & \rightarrow & \text { conical faces CAD and CBD } \\
\text { planar faces cfd and cgd } & \rightarrow & \text { tips A and B } \\
\text { cone } & \rightarrow & \text { top circular edge }
\end{array}
$$

For this example at least, the reciprocal transform method involves less calculations but more geometrical reasoning than the apparent contour method. The "filter" and its transform are shown in Fig. 22.

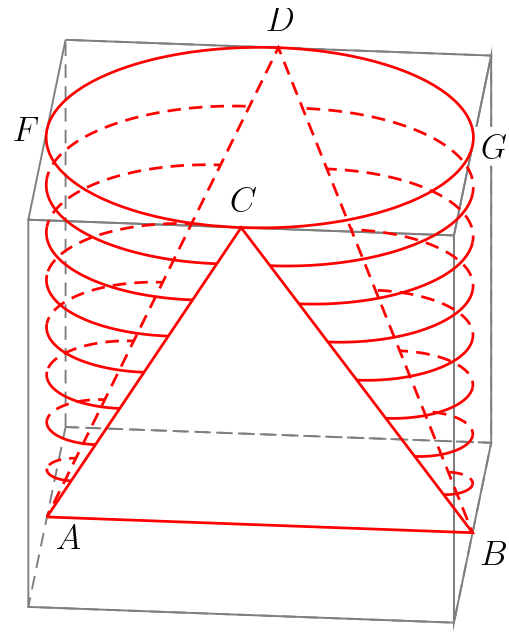

$\mathcal{D}_{3}$

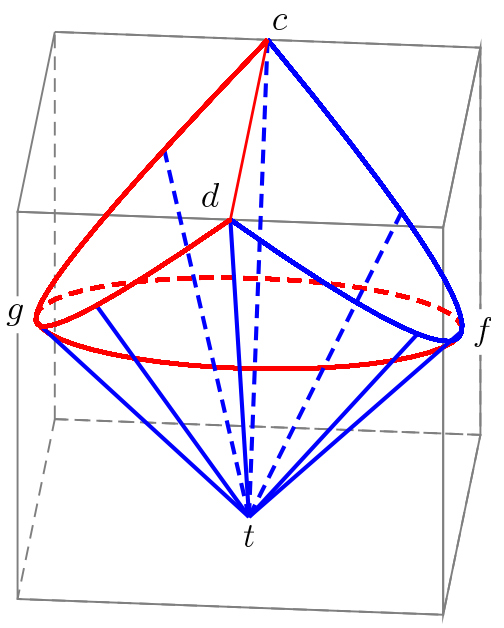

$\mathcal{I}_{3}$

Figure 22: Polar transform (right) of the funnel-shape filter (left) encountered as the allowed domain of some triples of observables, e.g., $\left\{P_{n}, C_{m m}, C_{n n}\right\}$. 


\subsubsection{Special cases}

The inequalities relating the spin observables of $\bar{p} p \rightarrow \bar{\Lambda} \Lambda$ have been derived without restriction on the underlying dynamics. As the early LEAR data indicated a clear dominance of spin-triplet transitions, the case where the spin-singlet is set to zero has been considered in [28].

Now, the formalism of $\bar{p} \mathrm{p} \rightarrow \bar{\Lambda} \Lambda$ applies without restriction to the reaction $\mathrm{e}^{-} \mathrm{e}^{+} \rightarrow \overline{\mathrm{p}} \mathrm{p}$, which is measured, e.g., at Frascati [101], and to the inverse reaction, which has been measured for instance at LEAR [102], and which is on the agenda of future antiproton facilities.

However, $\mathrm{e}^{-} \mathrm{e}^{+} \rightarrow \overline{\mathrm{p}} \mathrm{p}$ is dominated by the one-photon intermediate state in the $s$-channel. For this mechanism, in the more general case of the $\bar{B}_{1} B_{1} \rightarrow \bar{B}_{2} B_{2}$ reaction, the non-vanishing helicity amplitudes read (see, e.g., [103] for a recent compilation)

$$
\begin{aligned}
& \mathcal{H}_{++;++}=-\frac{2 \alpha m_{1} m_{2}}{s} \cos \vartheta G_{1}^{\mathrm{E}} G_{2}^{\mathrm{E}}, \\
& \mathcal{H}_{+-;+-}=-\frac{\alpha}{2}(1+\cos \vartheta) G_{1}^{\mathrm{M}} G_{2}^{\mathrm{M}}, \\
& \mathcal{H}_{+-;-+}=-\frac{\alpha}{2}(1-\cos \vartheta) G_{1}^{\mathrm{M}} G_{2}^{\mathrm{M}}, \\
& \mathcal{H}_{++;+-}=-\frac{\alpha m_{1}}{\sqrt{s}} \sin \vartheta G_{1}^{\mathrm{E}} G_{2}^{\mathrm{M}}, \\
& \mathcal{H}_{+-;++}=+\frac{\alpha m_{2}}{\sqrt{s}} \sin \vartheta G_{1}^{\mathrm{M}} G_{2}^{\mathrm{E}},
\end{aligned}
$$

where $\alpha$ is the fine-structure constant, $m_{1}$ and $m_{2}$ the mass of the baryon in the initial and final state, and $G_{i}^{\mathrm{E}, \mathrm{M}}$ their electric and magnetic form factors, which are complex in the time-like region, due to elastic and annihilation channels. Here, the $1 / s$ factor comes from the photon propagator, and each photon vertex is described as usual by a coupling

$$
\bar{u}\left[\gamma^{\mu} F(s)-\frac{i}{2 m} \sigma^{\mu \nu} q_{\nu} F^{\prime}(s)\right] u,
$$

the vector and tensor form factors being related to the usual electric and magnetic ones by

$$
G_{\mathrm{M}}=F+F^{\prime}, \quad G_{\mathrm{E}}=F+\frac{s}{4 m^{2}} F^{\prime} .
$$

In the case of an electron, $F=1$ and $F^{\prime}=0$.

The amplitudes $a, \ldots, g$ can be expressed in terms of these helicity amplitudes,

$$
\begin{aligned}
a & =\cos \vartheta\left(\mathcal{H}_{++;++}+\mathcal{H}_{++;--}+\mathcal{H}_{+-;+-}-\mathcal{H}_{+-;-+}\right) / 2+\sin \vartheta\left(-\mathcal{H}_{+;+-}+\mathcal{H}_{+-;++}\right), \\
b & =\left(\mathcal{H}_{++;++}-\mathcal{H}_{++;--}+\mathcal{H}_{+-;+-}+\mathcal{H}_{+-;-+}\right) / 2, \\
c & =\left(-\mathcal{H}_{++;++}+\mathcal{H}_{++;--}+\mathcal{H}_{+-;+-}+\mathcal{H}_{+-;-+}\right) / 2, \\
d & =\cos \vartheta\left(\mathcal{H}_{++;++}+\mathcal{H}_{++;--}-\mathcal{H}_{+-;+-}+\mathcal{H}_{+-;-+}\right) / 2+\sin \vartheta\left(\mathcal{H}_{++;+-}+\mathcal{H}_{+-;++}\right), \\
e & =-\cos \vartheta\left(\mathcal{H}_{++;+-}-\mathcal{H}_{+-;++}\right)-\sin \vartheta\left(\mathcal{H}_{++;++}+\mathcal{H}_{++;--}+\mathcal{H}_{+-;+-}-\mathcal{H}_{+-;-+}\right) / 2, \\
g & =\cos \vartheta\left(\mathcal{H}_{++;+-}+\mathcal{H}_{+-;++}\right)-\sin \vartheta\left(\mathcal{H}_{++;++}+\mathcal{H}_{++;--}-\mathcal{H}_{+-;+-}+\mathcal{H}_{+-;-+}\right) / 2 .
\end{aligned}
$$

The spin observables of the reaction $\mathrm{e}^{-} \mathrm{e}^{+} \rightarrow \overline{\mathrm{p}} \mathrm{p}$ are discussed in [104-106] ${ }^{12}$. It is particularly interesting to compare the values on and off the $\mathrm{J} / \psi$ peak.

\subsubsection{Proton-proton scattering and other similar reactions}

The results derived for $\bar{p} p \rightarrow \bar{\Lambda} \Lambda$ can be adapted to any $1 / 2+1 / 2 \rightarrow 1 / 2+1 / 2$ reaction. As already mentioned, the low-energy nucleon-nucleon observables have been measured in great detail,

\footnotetext{
${ }^{12} \mathrm{~A}$ detailed correspondence with T. Barnes and W. Roberts is gratefully acknowledged
} 
enabling one to reconstruct the five amplitudes in the each isospin channel, up to an overall phase. Hence the data are compatible with any possible model-independent inequality that can be devised beforehand.

The proton-proton exclusive reaction has also been studied at high energy. With the advent of QCD, the current wisdom based on perturbative contributions was that spin effects should generally be small as energy increases. However, a measurement by Krisch et al. [107-109], indicated a large value of the spin-spin correlation $A_{n n}$ at $90^{\circ}$, as a function of the incoming momentum. Their results are reproduced in Fig. 23. Also shown is the analysing power $A_{n}$, which should vanish at

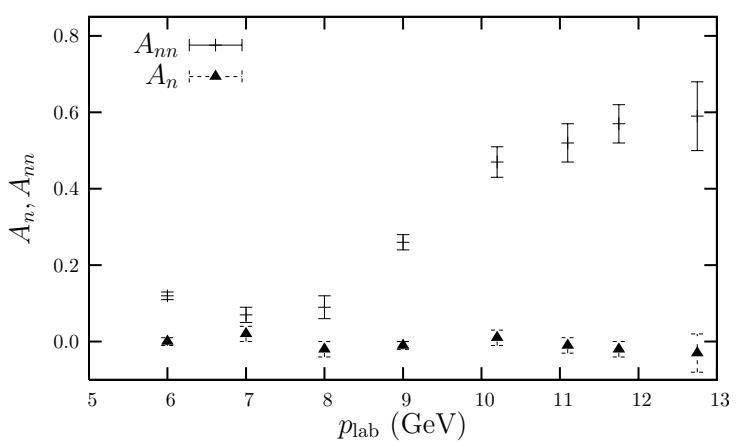

Figure 23: Analysing power $A_{n}$ and spinspin correlation $A_{n n}$ in proton-proton scattering at $90^{\circ}$, as a function of the beam momentum.

$90^{\circ}$, due to permutation symmetry.

This striking result of [107-109] motivated interesting studies on the underlying mechanisms. See, e.g., [65]. For our purpose, which is the allowed domain for observables, there is at $90^{\circ}$ a simplified situation, with only four independent helicity amplitudes, which, in the notation of [65], can be chosen as

$$
\alpha=M_{++,++}, \quad \beta=M_{+-,+-}=M_{-+,+-}, \quad \delta=M_{--,++},
$$

leading to the angular distribution $I_{0}=|\alpha|^{2}+2|\beta|^{2}+|\delta|^{2}$ and asymmetry coefficients given by

$$
\begin{aligned}
I_{0} A_{n n} & =2 \Re \mathrm{e}\left(\alpha^{*} \delta\right)+2|\beta|^{2}, \\
I_{0} A_{l l} & =|\alpha|^{2}+|\delta|^{2}-2|\beta|^{2}, \\
I_{0} A_{m m} & =-2 \Re \mathrm{e}\left(\alpha^{*} \delta\right)+2|\beta|^{2} .
\end{aligned}
$$

This implies the identity

$$
A_{n n}+A_{l l}+A_{m m}=1
$$

and the (redundant) inequalities

$$
A_{n n}+A_{l l}>0, \quad A_{n n}+A_{m m}>0, \quad A_{l l}+A_{m m}>0 .
$$

Unfortunately, a very large $A_{N N}$ does not constrain much the other asymmetry coefficients, for instance $A_{n n}=0.8$ leaves the interval $[-0.8,1]$ available for both $A_{l l}$ and $A_{m m}$.

For completeness, let us make the link with the notation used for $\overline{\mathrm{p} p} \rightarrow \bar{\Lambda} \Lambda$. In (3.15), $d=0$ for the equal-mass case such as $\bar{p} p \rightarrow \bar{p} p$ or $\mathrm{pp} \rightarrow \mathrm{pp}$. Furthermore at $90^{\circ}$ for pp elastic scattering, $a=0$ and $b=c$. If these relations are plugged into (3.17),

$$
C_{n n}+C_{l l}+C_{m m}=1,
$$

which is the analogue of (3.52), both already discussed in Sec. 2

Note that Gibbs and Loiseau [110] introduced a similar set of amplitudes, but following Ref. [5], they used unit vectors $\left\{\boldsymbol{\ell}^{\prime}, \boldsymbol{m}^{\prime}\right\}$ which are rotated by $45^{\circ}$ as compared to our $\{\boldsymbol{\ell}, \boldsymbol{m}\}$ for the scattered particle, so that their amplitudes are the same $\left(a^{\prime}=a\right.$, etc.), except for $c^{\prime}=-c, d^{\prime}=-g$ and $g^{\prime}=-d$. 


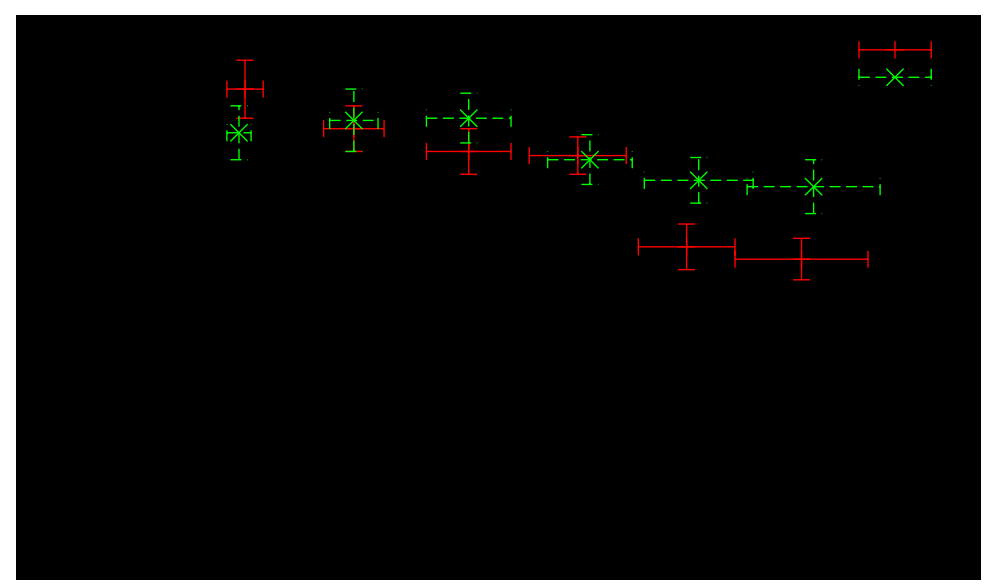

Figure 24: Beam asymmetry for $\gamma \mathrm{p} \rightarrow \eta \mathrm{p}$ at 2.5 and $3 \mathrm{GeV}$, as measured by Bussey et al. [112]

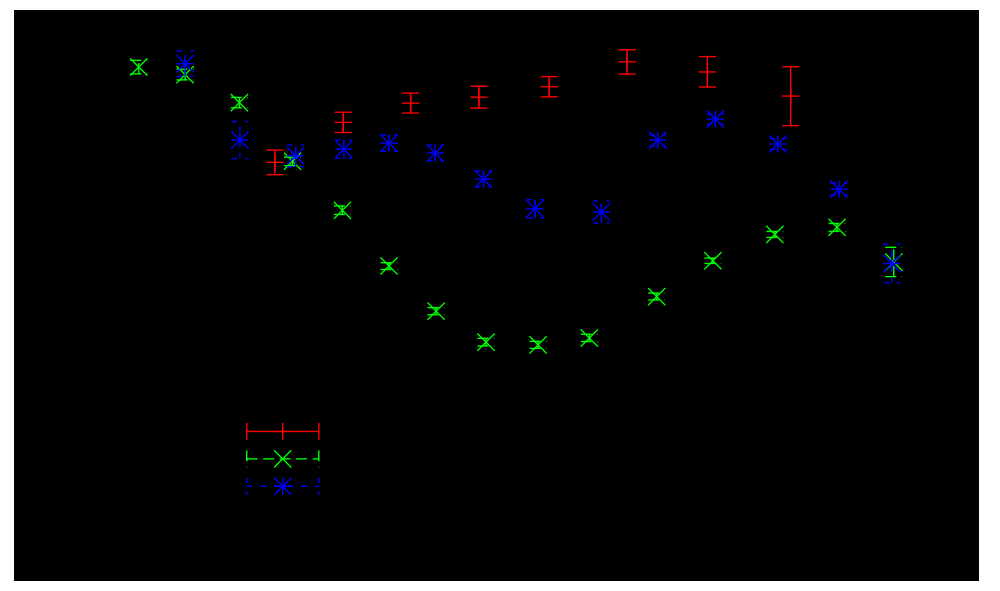

Figure 25: Beam asymmetry for $\gamma \mathrm{p} \rightarrow \pi^{0} \mathrm{p}$ at several photon energies, as measured by GRAAL [118].

\subsection{Photoproduction of scalar or pseudoscalar mesons}

\subsubsection{Experimental results}

There is an abundant literature on the reactions of photoproduction of a pseudoscalar meson, such as

$$
\gamma+\mathrm{N} \rightarrow \pi+\mathrm{N}, \quad \gamma+\mathrm{N} \rightarrow \mathrm{K}+\Lambda,
$$

and many data are available through the Durham data base [111]. Some interesting spin measurements have been performed many years ago, in particular with the aim of determining the spin and parity of new mesons, or to study the reaction mechanism. For instance, Bussey et al. [112] measured the beam asymmetry $\Sigma$ (to be defined shortly) of the reaction $\gamma \mathrm{p} \rightarrow \eta \mathrm{p}$ at 2.5 and $3 \mathrm{GeV}$, and found a value close to the maximum allowed, +1 , see Fig. 24. Large values of this observable $\Sigma$ were also found by the GRAAL collaboration [113].

Among the recent experiments are the spin measurements at LEPS [114], SAPHIR [115], GRAAL $[113,116]$ and CLAS [117]. In particular, the analogue of Fig. 24 for $\pi^{0}$, as measured by GRAAL [118], is reproduced in Fig. 25, for three of their energies (see [118] for the other data), showing again a large asymmetry at some angles. 
Much effort has also been devoted to the spin observables for $\gamma+\mathrm{N} \rightarrow \mathrm{K}+\Lambda$ at GRAAL and CLAS. The analysis of the CLAS data [119] has revealed the following approximate relation (see Fig. 26):

$$
P^{2}+C_{x}^{2}+C_{z}^{2} \simeq 1 .
$$

It means that when photon has pure circular polarisation, the $\Lambda$ hyperon is fully polarised. This relation will be discussed later in this section.

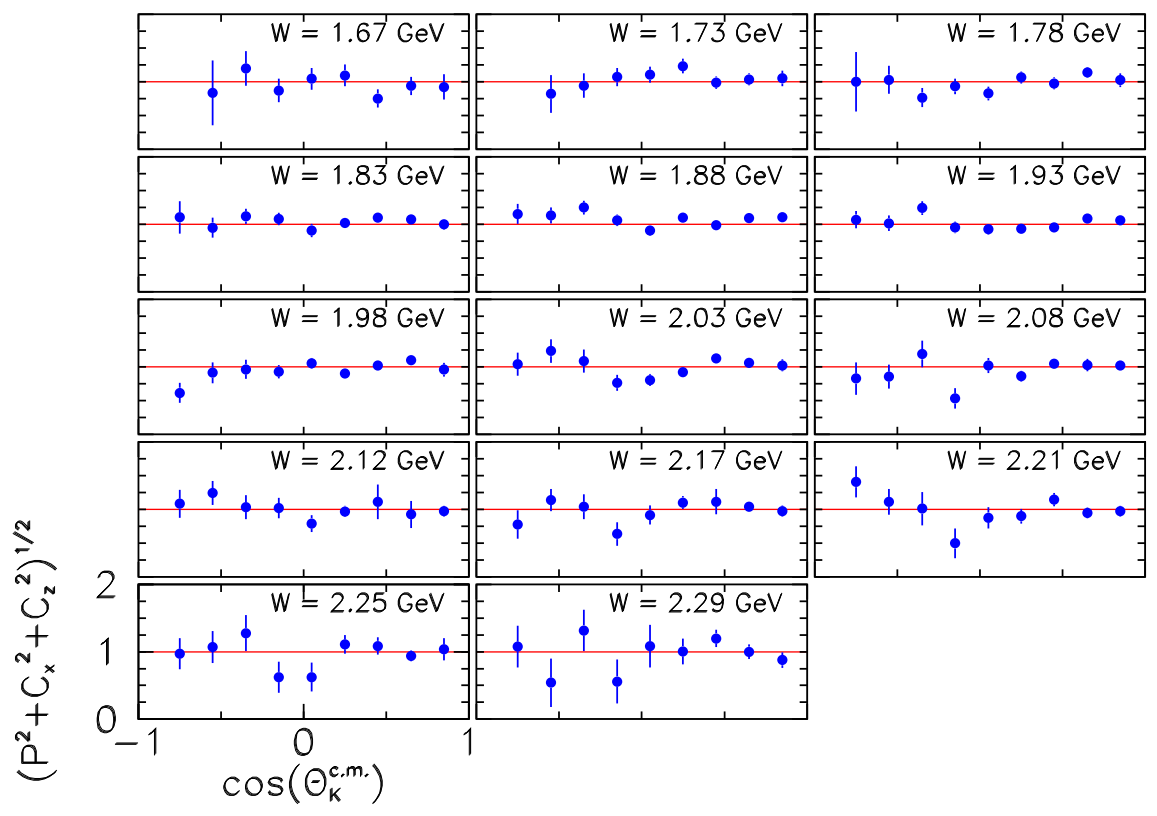

Figure 26: The magnitude of the $\Lambda$ hyperon polarisation observable vector $\sqrt{P^{2}+C_{x}^{2}+C_{z}^{2}}$ from recent CLAS data [119].

\subsubsection{Amplitudes}

In the pioneering paper by Chew et al. [120], the amplitude of the photoproduction of pseudoscalar mesons is written as a $2 \times 2$ matrix in the baryon spin space [120]

$$
\mathcal{M}=i \sigma . e F_{1}+\frac{\sigma \cdot \boldsymbol{q} \sigma \cdot(\boldsymbol{k} \times \boldsymbol{e})}{q k} F_{2}+i \frac{\sigma \cdot \boldsymbol{k} \boldsymbol{q} \cdot \boldsymbol{e}}{q k} F_{3}+i \frac{\sigma \cdot \boldsymbol{q} \boldsymbol{q} \cdot \boldsymbol{e}}{q^{2}} F_{4}
$$

where $e$ is the vector amplitude of the polarised photon, $\boldsymbol{k}$ and $\boldsymbol{q}$ are the initial and final relative momenta in the centre-of-mass system.

Since parity is conserved in this reaction, it is convenient to express the transition matrix $\mathcal{M}$ in the transversity basis: $|\boldsymbol{\pi}, \pm\rangle$ and $|\boldsymbol{n}, \pm\rangle$ for the initial state and $| \pm\rangle$ for the final state, where \pm denotes the transversity of the initial nucleon and of the final-state nucleon or hyperon, i.e., the projection $\pm 1 / 2$ of its spin along the normal to the scattering plane, and $\boldsymbol{\pi}$ (resp. $\boldsymbol{n}$ ) a photon state with linear polarisation parallel (resp. normal) to the scattering plane. These states are eigenstates of $\Pi$, the operator of reflection about the scattering plane. As explained in Sec. 2.8.2, one may assign 
$\Pi=+1$ for $+1 / 2$ transversity fermions and $\pi$-polarised photons, and $\Pi=-1$ for pseudoscalar mesons, $-1 / 2$ transversity fermions and $\boldsymbol{n}$-polarised photons. The four non-vanishing transversity amplitudes, which are independent, are

$$
\begin{array}{ll}
a_{1}=\langle+|\mathcal{M}| \boldsymbol{n}+\rangle, & a_{2}=\langle-|\mathcal{M}| \boldsymbol{n}-\rangle, \\
a_{3}=\langle+|\mathcal{M}| \boldsymbol{\pi}-\rangle, & a_{4}=\langle-|\mathcal{M}| \boldsymbol{\pi}+\rangle,
\end{array}
$$

while $\langle+|\mathcal{M}| \boldsymbol{n}-\rangle=\langle-|\mathcal{M}| \boldsymbol{n}+\rangle=\langle+|\mathcal{M}| \boldsymbol{\pi}+\rangle=\langle-|\mathcal{M}| \boldsymbol{\pi}-\rangle=0$. If a scalar meson was produced instead of a kaon, the sets of vanishing and non-vanishing transversity amplitudes would be interchanged.

The transversity amplitudes $a_{i}$ are related to the helicity ones [121], and can be expressed in terms of the CGLN amplitudes $F_{i}$ [122].

Several authors $[121,123,124]$ have considered the mathematical problem of fully reconstructing the amplitudes, up to an overall phase but without discrete ambiguities, from a well-chosen set of polarisation observables. Seven independent real functions can thus be determined. From all the possible experiments, one can extract sixteen different quantities, which are the bilinear products of the four amplitudes, i.e., of the seven real functions. These quantities are therefore not independent. However the relations between them are non-linear, so that measuring only seven of them leaves discrete ambiguities. The measurement of at least an eight quantity is necessary to resolves these ambiguities. Here we will not review this problem, but the linear and nonlinear equalities constraints coming from the positivity and rank one of the Cross Section Matrix, as in Ref. [125].

\subsubsection{The observables}

The various observables are traditionally classified and denoted as follows:

- the unpolarised differential cross section $I_{0}$,

- the linearly-polarised photon-beam asymmetry $\Sigma$ (sometimes denoted $\Sigma^{\gamma}$ or $\Sigma_{x}$ ),

- the polarised-target asymmetry $A$ (also denoted $A^{\mathrm{N}}$ ),

- the polarisation $P$ of the recoiling baryon (also denoted $P^{\mathrm{Y}}$ for $\gamma+\mathrm{N} \rightarrow \mathrm{K}+\mathrm{Y}$ ),

- the baryon depolarisation coefficients $T_{i}$ and $L_{i}$ expressing the correlation between the longitudinal or transverse (in the scattering plane) target polarisation and the spin of the recoil baryon,

- the coefficients describing the transfer of polarisation from a photon beam to the recoil baryon, in particular $O_{i}$ for oblique polarisation and $C_{i}$ for circular polarisation,

- the coefficients $G, H, E$ and $F$ of double spin correlations between the photon beam and the nucleon target,

- triple correlations coefficients if both the beam and the target are polarised and the hyperon polarisation analysed.

The index $i$ refers to the component in a frame $\{\hat{x}, \hat{y}, \hat{z}\}$ attached to each baryon: $\hat{y}$, the normal to the scattering plane, is the same for both, and $\hat{z}$ can be chosen along the centre-of-mass momentum $\boldsymbol{p}$, i.e., $\hat{z}=\boldsymbol{p} / p$ (in photoproduction experiments, $\hat{z}=-\boldsymbol{p} / p$ is usually chosen for the baryons). For the baryons, $S_{y}$ is the transversity. For the explicit calculations, it is convenient to choose a representation where $S_{y}$, instead of $S_{z}$, is diagonal. This leads to redefine the labelling of the Pauli matrices such that

$$
\sigma_{1}=\left(\begin{array}{ll}
0 & 1 \\
1 & 0
\end{array}\right)=\sigma_{z}, \quad \sigma_{2}=\left(\begin{array}{cc}
0 & -i \\
i & 0
\end{array}\right)=\sigma_{x}, \quad \sigma_{3}=\left(\begin{array}{cc}
1 & 0 \\
0 & -1
\end{array}\right)=\sigma_{y}
$$

with $\sigma_{1}$ corresponding to the helicity and $\sigma_{3}$ to the transversity of the baryon. For the photon, we use the Stokes parameters $\left(S^{1}, S^{2}, S^{3}\right)$ defined in Sec. 2.3: $S^{3} \equiv S_{\ominus}$ ("planarity") is the polarisation 
along $\hat{x}, S^{1} \equiv S_{\oslash}$ ("obliquity") is the polarisation along $(\hat{x}+\hat{y}) / \sqrt{2}$ and $S^{2} \equiv S_{\odot}$ is the circular polarisation, or helicity.

Using the general notation of Sec. 2, the fully polarised $(|\boldsymbol{S}|=1)$ differential cross section is expressed as

$$
\frac{d \sigma}{d \Omega}\left(\boldsymbol{S}_{\gamma}, \boldsymbol{S}_{\mathrm{N}}, \boldsymbol{S}_{\mathrm{Y}}\right)=I_{0}(\lambda \mu \mid \nu) S_{\gamma}^{\lambda} S_{\mathrm{N}}^{\mu} S_{\mathrm{Y}}^{\nu} .
$$

Here $\lambda, \mu, \nu$, run from 0 to 3 , the summation is understood over repeated indices and the polarisations have been promoted to four-vectors with $S^{0}=1$. The coefficients $(\lambda \mu \mid \nu)$ are related to the traditional ones by

$$
\begin{aligned}
& (00 \mid 0)=-(33 \mid 3)=1, \\
& \langle\ominus\rangle=-\left\langle y y^{\prime}\right\rangle=(30 \mid 0)=-(03 \mid 3)=-\Sigma, \\
& \langle y\rangle=-\left\langle\ominus y^{\prime}\right\rangle=(03 \mid 0)=-(30 \mid 3)=+A \text {, } \\
& \left\langle y^{\prime}\right\rangle=-\langle\ominus y\rangle=(00 \mid 3)=-(33 \mid 0)=+P, \\
& \left\langle z z^{\prime}\right\rangle=(01 \mid 1)=-(32 \mid 2)=+L_{z}, \\
& \left\langle z x^{\prime}\right\rangle=(01 \mid 2)=+(32 \mid 1)=+L_{x}, \\
& \left\langle x z^{\prime}\right\rangle=(02 \mid 1)=+(31 \mid 2)=+T_{z}, \\
& \left\langle x x^{\prime}\right\rangle=(02 \mid 2)=-(31 \mid 1)=+T_{x}, \\
& \langle\oslash z\rangle=(11 \mid 0)=+(22 \mid 3)=-G, \\
& \langle\oslash x\rangle=(12 \mid 0)=-(21 \mid 3)=-H, \\
& \langle\odot z\rangle=(21 \mid 0)=-(12 \mid 3)=+E, \\
& \langle\odot x\rangle=(22 \mid 0)=+(11 \mid 3)=+F, \\
& \left\langle\oslash z^{\prime}\right\rangle=(10 \mid 1)=-(23 \mid 2)=-O_{z}, \\
& \left\langle\oslash x^{\prime}\right\rangle=(10 \mid 2)=+(23 \mid 1)=-O_{x}, \\
& \left\langle\odot z^{\prime}\right\rangle=(20 \mid 1)=+(13 \mid 2)=+C_{z}, \\
& \left\langle\odot x^{\prime}\right\rangle=(20 \mid 2)=-(13 \mid 1)=+C_{x} \text {. }
\end{aligned}
$$

The symbol $\left\langle\oslash x^{\prime}\right\rangle$, for instance, is an intuitive notation for the correlation between the oblique polarisation of the photon (at $+\pi / 4$ ) and the polarisation towards $\hat{x}$ of the final baryon. The definition of $\Sigma, A, \ldots C_{x}$ is taken from [126]. They differ in sign with [121] concerning $L_{x}, G, E, C_{x}$ and $C_{z}$.

\subsubsection{The Cross Section Matrix}

The Cross Section Matrix of $\gamma+\mathrm{N}+\bar{\Lambda} \rightarrow \mathrm{K}$ is defined as

$$
\left\langle\boldsymbol{e}^{\prime}, s_{\mathrm{N}}^{\prime}, s_{\mathrm{Y}}^{\prime}|\mathcal{R}| \boldsymbol{e}, s_{\mathrm{N}}, s_{\mathrm{Y}}\right\rangle=\left\langle\boldsymbol{e}^{\prime}, s_{\mathrm{N}}^{\prime}\left|\mathcal{M}^{\dagger}\right| s_{\mathrm{Y}}^{\prime}\right\rangle\left\langle s_{\mathrm{Y}}|\mathcal{M}| \boldsymbol{e}, s_{\mathrm{N}}\right\rangle .
$$

By construction $\mathcal{R}$ is semi-positive definite and of rank 1. In terms of the observables for $\gamma+\mathrm{N} \rightarrow$ $\mathrm{K}+\Lambda$, the renormalised CSM reads

$$
\widehat{\mathcal{R}}=\left(2^{3} / \operatorname{Tr} \mathcal{R}\right) \mathcal{R}=(\lambda \mu \mid \nu) \sigma_{\gamma}^{\lambda} \otimes \sigma_{\mathrm{N}}^{\mu} \otimes\left[\sigma_{\mathrm{Y}}^{\nu}\right]^{t} .
$$

Note in (3.62) the crossing $\left|s_{Y}\right\rangle \leftrightarrow\left\langle s_{Y}\right|$ of the hyperon and the corresponding transposition of $\sigma_{Y}^{\nu}$ in (3.63)

In the transversity basis, owing to (3.58), $\widehat{\mathcal{R}}=\widehat{\mathcal{R}}^{+} \oplus \widehat{\mathcal{R}}^{-}$, where $\widehat{\mathcal{R}}^{-}$acts in the subspace spanned by

$$
|\boldsymbol{\pi}+-\rangle, \quad|\boldsymbol{\pi}-+\rangle, \quad|\boldsymbol{n}++\rangle \quad \text { and }|\boldsymbol{n}--\rangle,
$$

and $\widehat{\mathcal{R}}^{+}$acts on the complementary subspace. These matrices $\mathcal{R}^{-}$are given by Table 6 , where $(0+3,1-i 2 \mid 1+i 2)$, for instance, is a compact notation for

$$
(01 \mid 1)+(31 \mid 1)-i(02 \mid 1)-i(32 \mid 1)+i(01 \mid 2)+i(31 \mid 2)+(02 \mid 2)+(32 \mid 2) .
$$


Table 6: Submatrix $\widehat{\mathcal{R}}^{\mp}$ of the renormalised cross-section matrix $\widehat{\mathcal{R}}$. The upper sign refers to pseudoscalar photoproduction, the lower one to scalar.

\begin{tabular}{c|cccc} 
& $\pi+\mp$ & $\pi- \pm$ & $n+ \pm$ & $n-\mp$ \\
\hline$\pi+\mp$ & $(0+3,0+3 \mid 0 \mp 3)$ & $(0+3,1-i 2 \mid 1 \mp i 2)$ & $(1-i 2,0+3 \mid 1 \mp i 2)$ & $(1-i 2,1-i 2 \mid 0 \mp 3)$ \\
$\pi- \pm$ & $(0+3,1+i 2 \mid 1 \pm i 2)$ & $(0+3,0-3 \mid 0 \pm 3)$ & $(1-i 2,1+i 2 \mid 0 \pm 3)$ & $(1-i 2,0-3 \mid 1 \pm i 2)$ \\
$n+ \pm$ & $(1+i 2,0+3 \mid 1 \pm i 2)$ & $(1+i 2,1-i 2 \mid 0 \pm 3)$ & $(0-3,0+3 \mid 0 \pm 3)$ & $(0-3,1-i 2 \mid 1 \pm i 2)$ \\
$n-\mp$ & $(1+i 2,1+i 2 \mid 0 \mp 3)$ & $(1+i 2,0-3 \mid 1 \mp i 2)$ & $(0-3,1+i 2 \mid 1 \mp i 2)$ & $(0-3,0-3 \mid 0 \mp 3)$
\end{tabular}

Parity conservation. The 4 states of (3.64) correspond to the 4 helicity amplitudes which are allowed by parity for the photoproduction of a pseudoscalar meson. The other states correspond to vanishing amplitude, therefore $\mathcal{R}^{+}$vanishes identically. The reverse situation would hold for a scalar meson.

The 16 equalities in Eqs. (3.61) can be derived from $\mathcal{R}^{+}=0$. They correspond to the invariance of $(\lambda, \mu \mid \nu)$ under the substitutions: $0 \rightarrow-3,3 \rightarrow-0,1 \rightarrow i 2,2 \rightarrow-i 1$ for $\lambda$ and $\mu$ and $0 \rightarrow-3$, $3 \rightarrow-0,1 \rightarrow-i 2,2 \rightarrow i 1$ for $\nu$ and, in this way, they can be derived more quickly from

$$
\sigma_{3} \otimes \sigma_{3} \otimes \sigma_{3} \mathcal{R}=\mathcal{R} \sigma_{3} \otimes \sigma_{3} \otimes \sigma_{3}=-\mathcal{R}
$$

(cf. Eq. (2.101)). This Bohr identity comes from the vanishing of amplitudes between an even number of particles with negative $\sigma_{3}$.

Using these equalities, one can simplify $\hat{\mathcal{R}}$ by replacing $k \pm k^{\prime}$ (with $k=0$ or $1, k^{\prime}=3$ or 2 ) by $k$ once in each box of Table 6 . The result is equal to $\hat{\mathcal{R}} / 2$.

\subsubsection{Inequalities}

The positivity of the cross sections for normal polarisation of the baryon and $\boldsymbol{\pi}$ or $\boldsymbol{n}$-polarised photons (diagonal elements of Table 6) yields $[123,125]$

$$
|A-P| \leq 1-\Sigma, \quad|A+P| \leq 1+\Sigma .
$$

The domain corresponds to a tetrahedron schematically drawn in Fig. 27. Notice that its volume is

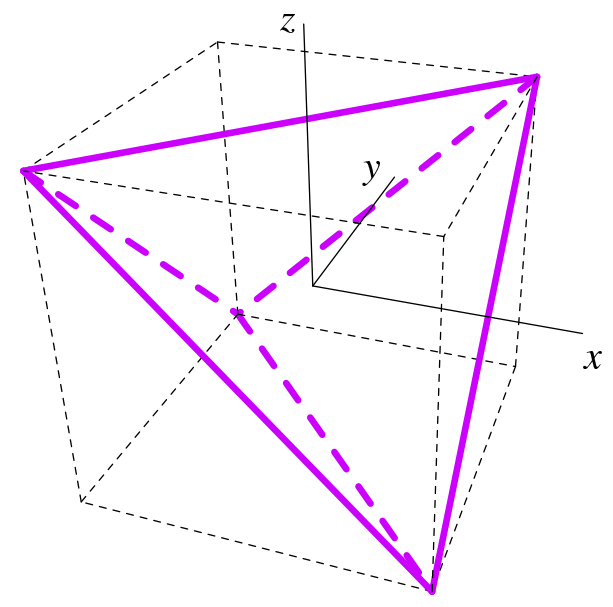

Figure 27: Tetrahedron domain limited by the inequalities (3.67) for the observables $x=A$, $y=P$ and $z=\Sigma$.

only $1 / 3$ of the volume of the entire cube, while its projection is the entire $[-1,+1]^{2}$ square on any face. Unit-ball domains are obtained for many triples like

$$
\left\{C_{x}, P, C_{z}\right\}, \quad\left\{O_{x}, P, O_{z}\right\}, \quad\left\{P, C_{x}, O_{x}\right\}, \quad\left\{P, C_{z}, O_{z}\right\} .
$$


They can be deduced from the anticommutation of the observables operators (see Sec. 2.6.2). But they have also classical meaning: For an unpolarised target and a photon Stokes vector $\left(S_{1}, S_{2}, S_{3}\right)$ the polarisation vector of the recoil baryon is $\left(S_{1} O_{x}+S_{2} C_{x}, P, S_{1} O_{z}+S_{2} C_{z}\right)$. The first triple of (3.68) contains the three components of that vector, when the photon is, say, right-handed $\left(S_{2}=1\right)$. Eq.(3.56) tells that the corresponding bound is experimentally saturated. Similarly for the second triple when the photon is oblique. For the third triple, let us introduce $a=\left[\left(O_{x}\right)^{2}+\left(C_{x}\right)^{2}\right]^{1 / 2}$. When the photon Stokes vector takes the particular value $\left(O_{x} / a, C_{x} / a, 0\right)$, the hyperon polarisation vector becomes $\left(a, P, O_{x} O_{z} / a+C_{x} C_{z} / a\right)$. Ignoring the last component we deduce $P^{2}+a^{2} \leq 1$, i.e., the third triple is in the unit ball. A similar demonstration holds for the fourth triple.

\subsubsection{Non-linear identities}

Many quadratic identities between observables can be derived from their expressions in terms of helicity or transversity amplitudes. A particularly elegant method [124] uses the Fierz identities of the Dirac algebra. Here, following the general method described in Sec. 2.7 (see also [125]), we will mainly use the fact that $\mathcal{R}$ (or $R^{-}$) has rank one, which implies the vanishing of all the $2 \times 2$ subdeterminants. We will also present more physical arguments, like the purity of the final polarisation when the initial particles are in pure states.

From the vanishing of the principal minors, one gets

$$
\begin{aligned}
& (1 \pm A)^{2}=(P \pm \Sigma)^{2}+\left(O_{z} \pm C_{x}\right)^{2}+\left(C_{z} \mp O_{x}\right)^{2}, \\
& (1 \pm P)^{2}=(A \pm \Sigma)^{2}+(G \mp F)^{2}+(E \pm H)^{2}, \\
& (1 \pm \Sigma)^{2}=(P \pm A)^{2}+\left(L_{z} \pm T_{x}\right)^{2}+\left(L_{x} \mp T_{z}\right)^{2} .
\end{aligned}
$$

These equations can also be obtained by simple physical arguments. For instance, if the target has pure transversity + and the photon in pure helicity + , then the hyperon spin components are

$$
S_{Y}^{j}=(0+2,0+3 \mid j) /(0+2,0+3 \mid 0)=\left(C_{z}-O_{x}, O_{z}+C_{x}, P+\Sigma\right) /(1+A) .
$$

Since the meson carries no spin information, the hyperon state must be pure, i.e., $\left|\boldsymbol{S}_{Y}\right|=1$. This gives (3.69a) with the sign + . One get the same identity if the photon is oblique.

From Eqs. (3.69), one gets

$$
\begin{aligned}
& 1+A^{2}=P^{2}+\Sigma^{2}+O_{z}^{2}+C_{x}^{2}+C_{z}^{2}+O_{x}^{2}, \\
& 1+P^{2}=A^{2}+\Sigma^{2}+G^{2}+F^{2}+E^{2}+H^{2}, \\
& 1+\Sigma^{2}=P^{2}+A^{2}+L_{z}^{2}+T_{x}^{2}+L_{x}^{2}+T_{z}^{2} .
\end{aligned}
$$

Complex identities, i.e., pairs of real identities, which do not contain $P, A$ nor $\Sigma$ can be obtained from the $2 \times 2$ determinants made only of non-diagonal elements of Table 1 , for instance

$$
(E+i G)^{2}+(F+i H)^{2}+\left(O_{z}-i C_{z}\right)^{2}+\left(O_{x}-i C_{x}\right)^{2}=0,
$$

from which one deduces

$$
0=E^{2}-G^{2}+F^{2}-H^{2}+O_{z}^{2}-C_{z}^{2}+O_{x}^{2}-C_{x}^{2} .
$$

From the combinations (3.71a)-(3.71b) $\pm(3.73)$,

$$
\begin{aligned}
& A^{2}+E^{2}+F^{2}=P^{2}+C_{x}^{2}+C_{z}^{2}, \\
& A^{2}+G^{2}+H^{2}=P^{2}+O_{x}^{2}+O_{z}^{2} .
\end{aligned}
$$

One can derive Eqs. (3.74) using crossing and basic results of quantum information theory. Consider the crossed reaction $\gamma+\bar{K} \rightarrow \bar{N}+\Lambda$ in which the $\gamma$ is fully polarised, e.g., in the +1 helicity state. Then the final $\bar{N}+\Lambda$ system is in a pure state. The individual density matrices $\rho_{N b}=\operatorname{Tr}_{\Lambda}\left(\rho_{\overline{\mathrm{N}}+\Lambda}\right)$ 
and $\rho_{\Lambda}=\operatorname{Tr}_{\overline{\mathrm{N}}}\left(\rho_{\bar{N}+\Lambda}\right)$ should have equal entropies, that is to say, the $\overline{\mathrm{N}}$ and $\Lambda$ polarisations are equal in magnitude:

$$
(0 \mid 30)^{2}+(2 \mid 10)^{2}+(2 \mid 20)^{2}=(0 \mid 03)^{2}+(2 \mid 01)^{2}+(2 \mid 02)^{2} .
$$

Crossing back to $\gamma+\mathrm{N} \rightarrow \Lambda+\mathrm{K}$, one changes $(\lambda \mid \mu \nu)^{2}$ into $(\lambda \mu \mid \nu)^{2}$ and obtains Eq. (3.74a). Equation (3.74b) is obtained in a similar way, considering a photon of oblique polarisation.

Inequalities can be recovered from the identities. Equation (3.69a), for instance, implies the eight spherical constraints

$$
1 \geq\left(\Sigma^{2} \text { or } P^{2}\right)+\left(O_{z}^{2} \text { or } C_{x}^{2}\right)+\left(C_{z}^{2} \text { or } O_{x}^{2}\right)
$$

four of which are already listed in (3.68). This can be seen from the inequality $\left(\boldsymbol{V}_{1}-\boldsymbol{V}_{2}\right)^{2} \leq$ $\left(\left|\boldsymbol{V}_{1}\right|+\left|\boldsymbol{V}_{2}\right|\right)^{2}$ applied to the vectors $\boldsymbol{V}_{1}=\left\{P+\Sigma, O_{z}+C_{x}, O_{x}+C_{z}\right\}$ and $\boldsymbol{V}_{2}=\left\{P-\Sigma, O_{z}-\right.$ $\left.C_{x}, O_{x}-C_{z}\right\}$ with normalization $\left|V_{1}\right|=1+A$ and $\left|V_{2}\right|=1-A$. A slight variant consists of reading (3.69a) as the four-vectors $\left.\left(1 \pm A ; P \pm \Sigma, O_{z} \pm C_{x}, O_{x} \pm C_{z}\right)\right)$ being light-like, and hence, since their time components $1 \pm A$ are positive, stating that their sum is time-like, in the same way as two photons combine into a massive state in elementary relativistic kinematics.

Very recently, Schumacher [127] stressed that in the CLAS data

$$
P^{2}+C_{x}^{2}+C_{z}^{2} \simeq 1 .
$$

This saturates the inequality $P^{2}+C_{x}^{2}+C_{z}^{2} \leq 1$ already encountered in (3.68) and (3.76). Then, using (3.71a), (3.74a) and (3.71b), one can predict

$$
\begin{aligned}
& \Sigma^{2}+O_{x}^{2}+O_{z}^{2} \simeq A^{2}, \\
& A^{2}+E^{2}+F^{2} \simeq 1, \\
& \Sigma^{2}+G^{2}+H^{2} \simeq P^{2} .
\end{aligned}
$$

The relation (3.78b) also means the saturation of an inequality.

\subsubsection{Symmetry rules}

Many other identities can be listed [124], but they are related by symmetry rules, in particular

- rotation in the $(1,2)$ plane, in particular the substitution $1 \rightarrow 2,2 \rightarrow-1$ for any particle. For a baryon, it corresponds to a change of $\{z, x\}$ axes in the scattering plane.

- permutation of the $\lambda, \mu, \nu$ indices, i.e., of the three particles with spin, except for the transposition of $\sigma_{Y}^{\nu}$. For instance, Eqs. (3.69a-3.69c) are related by such a transformation.

- $\pi / 2$ rotation in the $(1,3)$ plane: $1 \rightarrow 3,3 \rightarrow-1$ for the three particles simultaneously. This transformation violates the parity rules such as $(12 \mid 2)=0$ and the equalities of Eqs. (3.61). However, relaxing parity temporarily, we may apply it to a subdeterminant of $\mathcal{R}^{+}$in Table 6, then enforce parity conservation. A practical recipe does not need this table but uses the fact that any observable $\langle\mathcal{O}\rangle$ of (3.61) corresponds to two $(\lambda \mu \mid \nu)$ 's, one of which at least can be rotated without violating the parity rules. Applying this recipe to (3.69c), for instance, yields

$$
\left(1 \pm L_{z}\right)^{2}=\left(E \pm C_{z}\right)^{2}+\left(\Sigma \pm T_{x}\right)^{2}+\left(G \pm O_{z}\right)^{2},
$$

One can obtain this relations by considering the crossed reaction $\Lambda+\overline{\mathrm{N}} \rightarrow \gamma+\overline{\mathrm{K}}$, with $\Lambda$ and $\overline{\mathrm{N}}$ in pure helicity states and writing that the photon is fully polarised.

- substitution $0 \rightarrow i 1,1 \rightarrow i 0$ for all the particles, corresponding to an imaginary Lorentz transformation of the $\sigma_{\mu}$ 's in the $(0,1)$ plane. Equation $(3.69 \mathrm{c})$, for instance, is transformed into

$$
\left(1 \pm T_{x}\right)^{2}=\left(F \pm C_{x}\right)^{2}+\left(\Sigma \pm L_{z}\right)^{2}+\left(H \pm O_{x}\right)^{2} .
$$

One can interpret this relation with $\Lambda+\overline{\mathrm{N}} \rightarrow \gamma+\overline{\mathrm{K}}$ again, $\Lambda$ and $\overline{\mathrm{N}}$ having pure sideways polarisations. 


\subsubsection{Future applications}

At the time of the completion of this review, data are analysed by the CLAS and GRAAL collaboration, and for the first time, several spin observables will be accurately measured. An ongoing analysis [128] by Lleres et al., based on the identities and inequalities listed in [125] shows that the results on different observables for the same reaction $\gamma \mathrm{N} \rightarrow \mathrm{K} \Lambda$ are compatible.

A remarkable feature of our analysis is that, as for $\bar{p} p \rightarrow \bar{\Lambda} \Lambda$, there is no triple of observable whose allowed domain is the entire $[-1,+1]^{3}$ cube, as shown in Sec. 2.6.3. Every third observable is constrained by any pair of previously measured observable.

\subsection{Photoproduction of vector mesons}

\subsubsection{Experimental situation}

There are data on the reactions of the type

$$
\gamma+\mathrm{N} \rightarrow V+B
$$

with $V$ being a vector meson such as $\rho, \omega$ or $\phi$, and $B=\mathrm{N}^{\prime}$ a nucleon, or with $V$ being $\mathrm{K}^{*}$ and $B=Y$ an hyperon. The tensor polarisation is easily obtained, see (2.47-2.49), but there is no access to the vector polarisation of the meson. Note that the crossed reaction, $\gamma V \rightarrow \bar{p} p$, has the same spin structure as the photodisintegration of the deuteron, $\gamma \mathrm{d} \rightarrow \mathrm{pn}$, which is extensively studied at Jlab (see, e.g., [129]), and for which a formalism has been developed [130].

A summary of early experiments can be found in [131]. The photoproduction of $\rho, \omega$ and $\Phi$ mesons with a linearly-polarised photon beam has been studied by Ballam et al. [132] at 2.8, 4.7 and $9.3 \mathrm{GeV}$ beam energy. The reaction $\gamma+\mathrm{N} \rightarrow \mathrm{K}^{*}+\mathrm{Y}$ is studied at CLAS [133] for unpolarised photons. This collaboration also studied $\phi$ production [134]. Their results for the tensor-polarisation coefficient $\rho_{00}^{0}$ is shown in Fig. 28, with a large value at the highest transfer. The smallness of $\rho_{00}^{0}$ at low momentum transfer is also remarkable, and probably due to $s$-channel helicity conservation (an unpolarised vector meson has $\rho_{00}^{0}=1 / 3$ ). The density matrix $\rho_{\lambda \lambda^{\prime}}$ of the vector meson is expanded as a function of the possible photon polarisation $P_{k}$

$$
\rho_{\lambda \lambda^{\prime}} \propto \sum_{k=0}^{3} \rho_{\lambda \lambda^{\prime}}^{k} P_{k},
$$

where $k$ is the Stockes index, and $P_{0}=1$. The normalisation is specified later, see Eq. (3.100). For this reaction $\gamma+\mathrm{p} \rightarrow \phi+\mathrm{p}$, Halpern et al. [135] already noticed that the beam asymmetry is sometimes close to saturation, with a value $\Sigma=0.985 \pm 0.12$ for a photon energy $8.14 \mathrm{GeV}$ and a square transfer $t=-0.2(\mathrm{GeV} / c)^{2}$. The photoproduction of $\omega$ has been measured, e.g., by Ajaka et al. [136].

\subsubsection{Mechanisms}

In the review article [131], the data on $\rho$ photoproduction are interpreted in terms of Pomeron exchange, natural vs. unnatural-parity exchanges and $s$-channel helicity conservation. A comparison was also attempted between the photoproduction reaction (3.81) and the $\rho+\mathrm{N} \rightarrow V+B$ or $\omega+\mathrm{N} \rightarrow V+B$ reactions in the spirit of the vector-dominance model.

The language has of course evolved and data are now discussed in terms of quark dynamics. In particular, a comparison of spin effects between $\gamma+\mathrm{p} \rightarrow \phi+\mathrm{p}$ and $\gamma+\mathrm{p} \rightarrow \omega+\mathrm{p}$ is interesting to understand the mechanisms of violation of the OZI rule. It for instance could reveal a signature of a possible strangeness content of the nucleon [137]. 


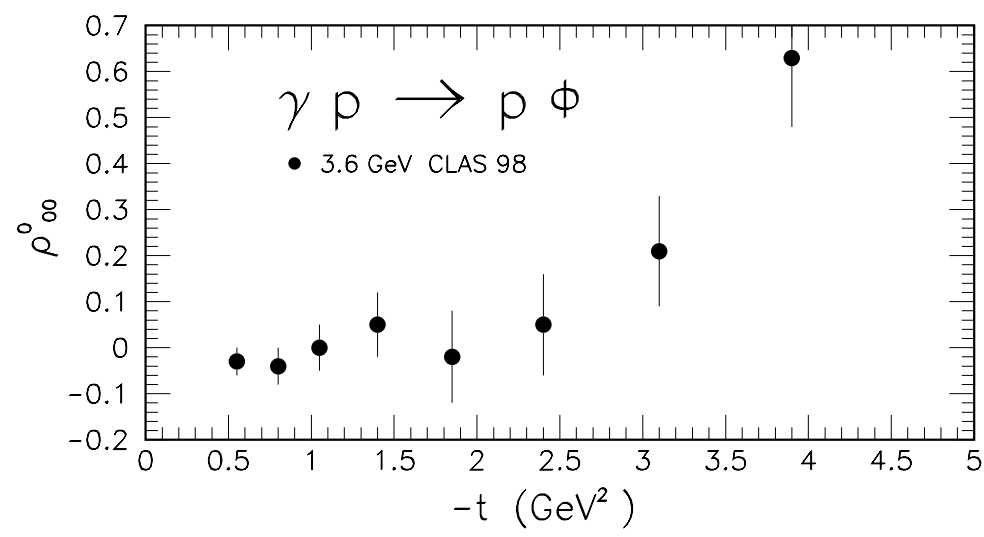

Figure 28: Tensor polarisation coefficient $\rho_{00}^{0}$ in $\gamma+\mathrm{p} \rightarrow \phi+\mathrm{p}$, as measured at CLAS [134].

\subsubsection{Formalism}

Kinematics The kinematics is shown in Fig. 29, with an explicit two-body decay mode $V \rightarrow$ $M_{1}+M_{2}$, which is the most current way of detecting a vector meson.

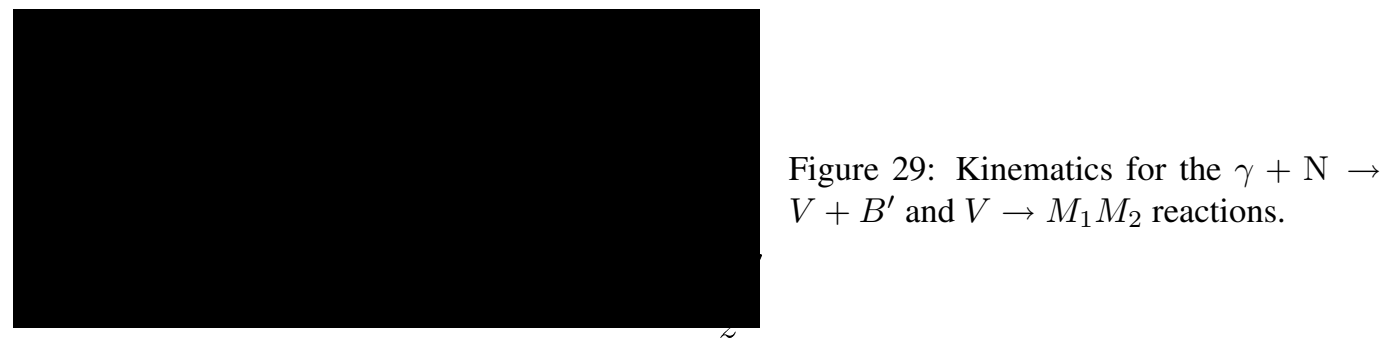

Helicity basis There are 24 helicity amplitudes, but this number reduces to 12 if parity is conserved

$$
\left\langle\lambda, \lambda_{B}|\mathcal{M}| \lambda_{\gamma}, \lambda_{\mathrm{N}}\right\rangle=(-1)^{\lambda-\lambda_{B}-\lambda_{\gamma}+\lambda_{N}}\left\langle-\lambda_{V}-\lambda_{B}|\mathcal{M}|-\lambda_{\gamma},-\lambda_{\mathrm{N}}\right\rangle .
$$

It is possible to chose $\lambda_{\gamma}=+1$ and use

$$
a_{i}=\left\langle 1, \lambda_{B}|\mathcal{M}| 1, \lambda_{N}\right\rangle, \quad b_{i}=\left\langle 0, \lambda_{B}|\mathcal{M}| 1, \lambda_{N}\right\rangle, \quad c_{i}=\left\langle-1, \lambda_{B}|\mathcal{M}| 1, \lambda_{N}\right\rangle,
$$

for the $\lambda= \pm 1,0$ helicities the vector meson, and for the baryons the index $i=1, \ldots 4$ defined as follows:

\begin{tabular}{c|cccc}
$i$ & 1 & 2 & 3 & 4 \\
\hline$\lambda_{B}$ & $+1 / 2$ & $+1 / 2$ & $-1 / 2$ & $-1 / 2$ \\
$\lambda_{\mathrm{N}}$ & $-1 / 2$ & $+1 / 2$ & $-1 / 2$ & $+1 / 2$
\end{tabular}

The various spin observables can be expressed in terms of these amplitudes $H_{a \lambda}$. For instance,

$$
\begin{aligned}
I_{0} & =\left|a_{1}\right|^{2}+\left|b_{1}\right|^{2}+\cdots+\left|b_{4}\right|^{2}+\left|c_{4}\right|^{2}, \\
I_{0} \Sigma & =-2 \Re \mathrm{e}\left(c_{1} a_{4}^{*}-b_{1} b_{4}^{*}+a_{1} c_{4}^{*}-c_{2} a_{3}^{*}+b_{2} b_{3}^{*}-a_{2} c_{3}^{*}\right), \\
I_{0} T & =-2 \Im \mathrm{m}\left(c_{3} c_{4}^{*}+b_{3} b_{4}^{*}+a_{3} a_{4}^{*}+c_{1} c_{2}^{*}+b_{1} b_{2}^{*}+a_{1} a_{2}^{*}\right),
\end{aligned}
$$

for the angular distribution, beam asymmetry and target analysing power. See, e.g., [137, 138]. In [137], all single and double polarisation observables are listed, and expressed in terms of the above helicity amplitudes. 
Transversity and linear polarisation bases For the initial and final baryons, and for the photon, we use the same notation \pm and $\{\boldsymbol{\pi}, \boldsymbol{n}\}$ for the transversity states as in Sec. 3.4.2. For the vector meson, we will take the linearly polarised basis $\{|x\rangle,|y\rangle,|z\rangle\}$. Assuming that $\mathrm{V}$ has the natural parity $P=-1$, the states $|x\rangle$ and $|z\rangle$ polarised in the scattering plane have $\Pi_{\mathrm{V}}=+1$ whereas $|z\rangle$ has $\Pi_{\mathrm{V}}=-1$. The conservation of $\Pi$, which reads

$$
\Pi_{\gamma} \Pi_{\mathrm{N}}=\Pi_{\mathrm{B}} \Pi_{\mathrm{V}}
$$

selects 12 non-vanishing transversity amplitudes (out of 24), which can be chosen as:

$$
\begin{array}{rlrl}
\langle x,+|\mathcal{M}| \boldsymbol{\pi},+\rangle & =f_{1}, & & \langle x,-|\mathcal{M}| \boldsymbol{n},+\rangle=g_{1}, \\
\langle x,-|\mathcal{M}| \boldsymbol{\pi},-\rangle=f_{2}, & & \langle x,+|\mathcal{M}| \boldsymbol{n},-\rangle=g_{2}, \\
\langle z,+|\mathcal{M}| \boldsymbol{\pi},+\rangle=f_{3}, & & \langle z,-|\mathcal{M}| \boldsymbol{n},+\rangle=g_{3}, \\
\langle z,-|\mathcal{M}| \boldsymbol{\pi},-\rangle=f_{4}, & & \langle z,+|\mathcal{M}| \boldsymbol{n},-\rangle=g_{4}, \\
\langle y,+|\mathcal{M}| \boldsymbol{n},+\rangle=f_{5}, & & \langle y,-|\mathcal{M}| \boldsymbol{\pi},+\rangle=g_{5}, \\
\langle y,-|\mathcal{M}| \boldsymbol{n},-\rangle=f_{6}, & & \langle y,+|\mathcal{M}| \boldsymbol{\pi},-\rangle=g_{6} .
\end{array}
$$

The $f_{i}\left(\right.$ resp. $g_{i}$ ) amplitudes correspond to $\Pi_{\mathrm{V}}=\Pi_{\gamma}, \Pi_{\mathrm{B}}=\Pi_{\mathrm{N}}\left(\right.$ resp. $\Pi_{\mathrm{V}}=-\Pi_{\gamma}, \Pi_{\mathrm{B}}=-\Pi_{\mathrm{N}}$ ), that is to say, natural (resp. unnatural) parity exchange in the $t$-channel.

The cross section matrix. The full spin dependence of the reaction is encoded in the cross section matrix $\mathcal{R}$ or its partial transpose $\widetilde{\mathcal{R}}$ defined by (2.68). Equations (2.69) and (2.73) are generalised as

$$
\begin{aligned}
(\lambda \mu \mid \alpha \nu) & =\operatorname{Tr}\left\{\widetilde{\mathcal{R}}\left(\sigma_{\lambda}^{\gamma} \otimes \sigma_{\mu}^{\mathrm{N}} \otimes \Sigma_{\alpha} \otimes \sigma_{\nu}^{B}\right)\right\}, \\
\widetilde{\mathcal{R}} /(\operatorname{Tr} \widetilde{\mathcal{R}}) & =(\lambda \mu \mid \alpha \nu) 2^{-3} g_{\alpha}^{-1} \sigma_{\lambda}^{\gamma} \otimes \sigma_{\mu}^{\mathrm{N}} \otimes \Sigma_{\alpha}^{\dagger} \otimes \sigma_{\nu}^{B},
\end{aligned}
$$

where $\lambda, \mu, \nu \in\{0,1,2,3\}, \Sigma_{\alpha}$ are nine basic spin-1 operators ${ }^{13}$ and $g_{\alpha}=\operatorname{Tr}\left(\Sigma_{\alpha} \Sigma_{\alpha}^{\dagger}\right)$. Here we will simply take the basis described by (2.36).

$\mathcal{R}$ has rank 1 and effective dimension $12 \times 12$. Hence the spin observables are expressed in terms of 144 linearly independent (but non-linearly dependent) parameters. Only $2 \times 12-1=23$ parameters are linearly and quadratically independent.

Among the $4 \times 4 \times 4 \times 9=576$ observables, half of them vanish due to the classical constraints of parity (2.95-2.96). They involve an odd number of $\Pi$-odd single particle operators in (3.89). The $\Pi$-odd operators are $\sigma_{1}$ and $\sigma_{2}$ for the photon and the baryons, $|x\rangle\langle y|| y\rangle,\langle x|| z\rangle,\langle y|$ and $|y\rangle\langle z|$ for the vector meson. Each vector-meson operator $\Sigma_{\alpha}$ is present in $4 \times 4 \times 2=32$ parity-allowed observables.

The non-classical constraint (2.100) relates the nonvanishing observables two-by-two (in our basis of observables), up to a sign. For instance $(1,0 \mid y y, 1)=-(2,3 \mid y y, 2), \quad(3,1 \mid y z, 3)=$ $+(0,2 \mid y z, 0),(3,1 \mid z y, 3)=-(0,2 \mid z y, 0)$. This divides by 2 the number of linearly independent observables, which are therefore 144 , as already predicted. For the $\rho, \omega, \Phi$ and $\mathrm{K}^{*}$ mesons, however, only the tensor polarisation can be measured through the angular distribution of the decay products and the axial polarisation $\Im \mathrm{m} \rho_{i i^{\prime}}$ is hidden. This reduces to 128 the number of linearly independent accessible observables. In what follows we consider successively the cases where one, two and the three spin-doublet particles (i.e., the photon, the nucleon and the recoil baryon) are unpolarised or non-analysed.

One unpolarised or non-analysed spin-doublet particle. Let us assume for instance that the final baryon is not analysed: only observables involving $\sigma_{0}^{(\mathrm{B})}$ are measured. They are all linearly

\footnotetext{
${ }^{13}$ The appearance of $\Sigma^{\dagger}$ in (3.90) is analogous to what happens between (2.34) and (2.35) using the orthogonality condition (2.33)
} 
independent since the identity (2.100) relates them to non-measured observables involving $\sigma_{3}^{(\mathrm{B})}$. For each vector-meson operator $\Sigma_{\alpha}$ there is $4 \times 2=8$ measured observables. This gives $6 \times 8=48$ linearly independent accessible observables $(9 \times 8=72$ if the axial polarisation of $V$ was not hidden). The reduced cross section matrix for this experiment is

$$
\mathcal{R}_{\gamma \mathrm{N}, \mathrm{V}} \propto(\lambda \mu \mid \alpha 0) g_{\alpha}^{-1} \sigma_{\lambda}^{\gamma} \otimes \sigma_{\mu}^{\mathrm{N}} \otimes\left(\Sigma_{\alpha}^{\dagger}\right)^{t}
$$

It is block-diagonal in two $6 \times 6$ matrices. The blocks $\mathcal{R}_{\gamma \mathrm{N}, \mathrm{V}}^{+}$and $\mathcal{R}_{\gamma \mathrm{N}, \mathrm{V}}^{-}$correspond to the final baryon having transversity $+1 / 2$ and $-1 / 2$ respectively. With the convention $\eta_{\mathrm{B}}=i$ we have

$$
\Pi \mathcal{R}_{\gamma \mathrm{N}, \mathrm{V}}^{ \pm}=\mathcal{R}_{\gamma \mathrm{N}, \mathrm{V}}^{ \pm} \Pi= \pm \mathcal{R}_{\gamma \mathrm{N}, \mathrm{V}}^{ \pm}
$$

$\mathcal{R}_{\gamma \mathrm{N}, \mathrm{V}}^{ \pm}$has 36 linearly independent parameters. It is of rank one, since it corresponds to one photon state. So, in a non-linear way, it depends only on $2 \times 6-1=11$ parameters.

Two unpolarised or non-analysed spin-doublet particles Let us now study the case where $\mathrm{N}$ is unpolarised, $B$ not analysed. For each vector-meson operator $\Sigma_{\alpha}$ there is 2 measured observables. This gives $6 \times 2=12$ linearly independent accessible observables $(9 \times 2=18$ if the axial polarisation of $V$ were not hidden). The reduced density matrix is

$$
\mathcal{R}_{\gamma, V} \propto(\lambda 0 \mid \alpha 0) g_{\alpha}^{-1} \sigma_{\lambda}^{\gamma} \otimes\left(\Sigma_{\alpha}^{\dagger}\right)^{t} .
$$

It is block-diagonal in two $3 \times 3$ matrices $\mathcal{R}_{\gamma, V}^{+}$and $\mathcal{R}_{\gamma, V}^{-}$obeying equations analogous to (3.92) and corresponding respectively to $\mathrm{N}$ and $B$ having equal or opposite transversities. In each case, the $(\mathrm{N} \bar{B})$ system in the $t$-channel is two-dimensional, so that $\mathcal{R}_{\gamma, V}^{+}$and $\mathcal{R}_{\gamma, V}^{-}$have rank 2 . Each of them has 9 linearly independent parameters, but related by $\operatorname{det}\left(\mathcal{R}_{\gamma, V}^{ \pm}\right)=0$, therefore 8 really independent parameters. We will come back to this case for more details in Sec. 3.5.4.

No polarised or analysed spin-doublet particle. Repeating the reduction once again we arrive at the $3 \times 3$ density matrix of the vector meson, when all other spins are summed over:

$$
\widetilde{\mathcal{R}}_{V}=\mathcal{R}_{V}^{t} \propto \rho=(00 \mid \alpha 0) g_{\alpha}^{-1} \Sigma_{\alpha} .
$$

$\rho$ is the direct sum of a $2 \times 2$ matrix $\rho^{+}$acting on the $\Pi_{\mathrm{V}}=+1$ subspace $\{a|x\rangle+b|z\rangle\}$ (linear polarisation in the scattering plane) and the simple number $\rho_{y y}$ acting on the $\Pi_{\mathrm{V}}=-1$ subspace colinear to $|y\rangle$ :

$$
\left(\rho_{i j}\right)=\left(\begin{array}{ccc}
\rho_{x x} & 0 & \rho_{x z} \\
0 & \rho_{y y} & 0 \\
\rho_{z x} & 0 & \rho_{z z}
\end{array}\right) .
$$

The dimension 4 of the $(\gamma \mathrm{N} \bar{B})$ channel for $\Pi=+1$ does not restricts the rank of $\rho^{+}$, so that $\rho_{x x}, \rho_{z z}, \Re \mathrm{e} \rho_{x z}$ and $\Im \mathrm{m} \rho_{x z}$ are fully independent parameters. Note that the latter is generally not accessible. The positivity conditions are

$$
\left.\rho_{x x} \geq 0, \quad \rho_{y y} \geq 0, \quad \rho_{z z} \geq 0, \quad\left(\Re \mathrm{e} \rho_{x z}\right)^{2}+\left(\Im \mathrm{m} \rho_{x z}\right)\right)^{2} \leq \rho_{x x} \rho_{z z} .
$$

\subsubsection{Inequalities for the case of unpolarised baryons}

We return to the case $\mathrm{N}$ unpolarised, $B$ not analysed, where experimental data exist [132]. Writing (3.93) with the $\Sigma_{\left\{i i^{\prime}\right\}}$ operator basis, we have

$$
\widehat{\mathcal{R}} \equiv 2 \mathcal{R}_{\gamma, V} /\left(\operatorname{Tr} \mathcal{R}_{\gamma, V}\right)=\left(\lambda \mid i i^{\prime}\right) \sigma_{\lambda}^{\gamma} \otimes\left(\left|i^{\prime}\right\rangle\langle i|\right),
$$


where $(\lambda, 0 \mid \alpha, 0)=\left(\lambda, 0 \mid\left\{i i^{\prime}\right\}, 0\right)$ has been abbreviated as $\left(\lambda \mid i i^{\prime}\right)$. The matrix $\widehat{\mathcal{R}}$ is normalised such that $\left(0 \mid i i^{\prime}\right)$ is equal to the density matrix $\rho_{i i^{\prime}}^{0}$ of the vector meson when the photon is unpolarised. Its explicit expression is

$$
\left(\begin{array}{cccccc}
(0+3 \mid x x) & 0 & (0+3 \mid z x) & 0 & (1-i 2 \mid y x) & 0 \\
0 & (0+3 \mid y y) & 0 & (1-i 2 \mid x y) & 0 & (1-i 2 \mid z y) \\
(0+3 \mid x z) & 0 & (0+3 \mid z z) & 0 & (1-i 2 \mid y z) & 0 \\
0 & (1+i 2 \mid y x) & 0 & (0-3 \mid x x) & 0 & (0-3 \mid z x) \\
(1+i 2 \mid x y) & 0 & (1+i 2 \mid z y) & 0 & (0-3 \mid y y) & 0 \\
0 & (1+i 2 \mid y z) & 0 & (0-3 \mid x z) & 0 & (0-3 \mid z z)
\end{array}\right)
$$

The linear polarisation basis for the photon + vector meson states has been ordered as $|x\rangle \otimes|x\rangle$, $|x\rangle \otimes|y\rangle,|x\rangle \otimes|z\rangle,|y\rangle \otimes|x\rangle,|y\rangle \otimes|y\rangle,|y\rangle \otimes|z\rangle$. The notation $(1-i 2 \mid x y)$, for instance, stands for $(1 \mid x y)-i(2 \mid x y)$. Adopting a notation similar to that of Schilling et al. [52] we rewrite $(0 \mid i j)$, $(1 \mid i j),(2 \mid i j)$ and $(3 \mid i j)$ as $\rho_{i j}^{0}, \rho_{i j}^{\ominus}, \rho_{i j}^{\odot}$ and $\rho_{i j}^{\ominus}$. The beam asymmetry is

$$
\Sigma=\frac{\operatorname{Tr}\left\{\mathcal{R}\left(\sigma_{\ominus}^{\gamma} \otimes \mathbb{1}^{V}\right)\right\}}{\operatorname{Tr} \mathcal{R}}=\rho_{x x}^{\ominus}+\rho_{y y}^{\ominus}+\rho_{z z}^{\ominus} .
$$

The meson density matrix takes the form

$$
\rho=\frac{1}{1+S_{\ominus} \Sigma} \sum_{\lambda=0, \oslash, \odot, \ominus} \rho^{\lambda} S_{\lambda}
$$

Note that $\rho$ depends nonlinearly on the photon Stokes parameter $S_{\ominus}$, due to the denominator which is inversely proportional to the cross section with polarised photons. Schilling et al. [52] use the helicity basis and their Stokes parameters, $\left\{P_{1}, P_{2}, P_{3}\right\}=\left\{-S_{\ominus},-S_{\oslash}, S_{\odot}\right\}$ are different from ours, $\left\{S_{1}, S_{2}, S_{3}\right\}=\left\{S_{\ominus}, S_{\odot}, S_{\ominus}\right\}$. We give here the correspondence between their notations (lefthand side) and ours (right-hand side) for the accessible observables:

$$
\begin{aligned}
\left(1-\rho_{00}^{0}\right) / 2=\rho_{11}^{0}=\rho_{-1-1}^{0} & \leftrightarrow\left(\rho_{x x}^{0}+\rho_{y y}^{0}\right) / 2=\left(1-\rho_{z z}^{0}\right) / 2 \\
\rho_{1,-1}^{0}=\rho_{-1,1}^{0} & \leftrightarrow\left(-\rho_{x x}^{0}+\rho_{y y}^{0}\right) / 2 \\
\Re \mathrm{e} \rho_{10}^{0}=-\Re \mathrm{e} \rho_{0,-1}^{0} & \leftrightarrow-\Re \mathrm{e} \rho_{z x}^{0} / \sqrt{2} \\
\rho_{00}^{1} & \leftrightarrow-\rho_{z z}^{\ominus} \\
\rho_{11}^{1}=\rho_{-1-1}^{1} & \leftrightarrow-\left(\rho_{x x}^{\ominus}+\rho_{y y}^{\ominus}\right) / 2 \\
\rho_{1,-1}^{1}=\rho_{-1,1}^{1} & \leftrightarrow\left(\rho_{x x}^{\ominus}-\rho_{y y}^{\ominus}\right) / 2 \\
\Re \mathrm{e} \rho_{1,0}^{1}=-\Re \mathrm{e} \rho_{-1,0}^{1} & \leftrightarrow \Re \mathrm{e} \rho_{z x}^{\ominus} / \sqrt{2} \\
\rho_{1,-1}^{2} & \leftrightarrow-i \Re \mathrm{e} \rho_{x y}^{\ominus} \\
\Im \mathrm{m} \rho_{10}^{2}=\Im \mathrm{m} \rho_{-1,0}^{2} & \leftrightarrow-\Re \mathrm{e} \rho_{y z}^{\ominus} / \sqrt{2} \\
\rho_{1,-1}^{3} & \leftrightarrow i \Re \mathrm{e} \rho_{x y}^{\odot} \\
\Im \mathrm{m} \rho_{10}^{3}=\Im \mathrm{m} \rho_{-1,0}^{3} & \leftrightarrow \Re \mathrm{e} \rho_{y z}^{\odot} / \sqrt{2} .
\end{aligned}
$$

As predicted above, the matrix $\widehat{\mathcal{R}}_{\gamma, V}$ given by Eq. (3.98) is block-diagonal in two semi-positive $3 \times 3$ matrices of rank 2: $\widehat{\mathcal{R}}_{\gamma, V}^{+}$, made of the odd lines and columns, is associated to equal transversities of the initial and final baryons and to natural parity exchange in the $t$-channel. $\widehat{\mathcal{R}}_{\gamma, V}^{-}$, made of the even lines and columns, is associated to a baryon transversity flip and unnatural parity exchange. For instance

$$
R_{\gamma, V}^{+}=\left(\begin{array}{ccc}
(0 \mid x x)+(3 \mid x x) & (0 \mid z x)+(3 \mid z x) & (1 \mid y x)-i(2 \mid y x) \\
(0 \mid x z)+(3 \mid x z) & (0 \mid z z)+(3 \mid z z) & (1 \mid y z)-i(2 \mid y z) \\
(1 \mid x y)+i(2 \mid x y) & (1 \mid z y)+i(2 \mid z y) & (0 \mid y y)-(3 \mid y y)
\end{array}\right)
$$


The vanishing of the determinants of $\mathcal{R}_{\gamma, V}^{ \pm}$yield two cubic relations between the observables. We do not write them here, since they involve the axial polarisation of the vector meson, which is not usually accessible. The positivity of the upper-left $2 \times 2$ minor of (3.102), and a similar condition for $\widehat{\mathcal{R}}_{\gamma, V}^{-}$, read

$$
\left(\Re \mathrm{e}\left[\rho_{z x}^{0} \pm \rho_{z x}^{\ominus}\right]\right)^{2}+\left(\Im \mathrm{m}\left[\rho_{z x}^{0} \pm \rho_{z x}^{\ominus}\right]\right)^{2} \leq\left(\rho_{x x}^{0} \pm \rho_{x x}^{\ominus}\right)\left(\rho_{z z}^{0} \pm \rho_{z z}^{\ominus}\right) .
$$

These two inequalities are necessary conditions for the positivity of the meson density matrix when the photon is polarised in the scattering plane. They are therefore classical positivity conditions. The positivity of the other $2 \times 2$ principal minors of $\mathcal{R}_{\gamma, V}^{ \pm}$yield the non-classical conditions

$$
\begin{aligned}
{\left[\Re \mathrm{e} \rho_{y x}^{\ominus}+\Im \mathrm{m} \rho_{y x}^{\odot}\right]^{2}+\left[\Im \mathrm{m} \rho_{y x}^{\ominus}-\Re \mathrm{e} \rho_{y x}^{\odot}\right]^{2} \leq\left(\rho_{x x}^{0}+\rho_{x x}^{\ominus}\right)\left(\rho_{y y}^{0}-\rho_{y y}^{\ominus}\right), } \\
{\left[\Re \mathrm{e} \rho_{y z}^{\ominus}+\Im \mathrm{m} \rho_{y z}^{\odot}\right]^{2}+\left[\Im \mathrm{m} \rho_{y z}^{\ominus}-\Re \mathrm{e} \rho_{y z}^{\odot}\right]^{2} \leq\left(\rho_{z z}^{0}+\rho_{z z}^{\ominus}\right)\left(\rho_{y y}^{0}-\rho_{y y}^{\ominus}\right), } \\
{\left[\Re \mathrm{e} \rho_{x y}^{\ominus}+\Im \mathrm{m} \rho_{x y}^{\odot}\right]^{2}+\left[\Im \mathrm{m} \rho_{x y}^{\ominus}-\Re \mathrm{e} \rho_{x y}^{\odot}\right]^{2} \leq\left(\rho_{x x}^{0}-\rho_{x x}^{\ominus}\right)\left(\rho_{y y}^{0}+\rho_{y y}^{\ominus}\right), } \\
{\left[\Re \mathrm{e} \rho_{z y}^{\ominus}+\Im \mathrm{m} \rho_{z y}^{\odot}\right]^{2}+\left[\Im \mathrm{m} \rho_{z y}^{\ominus}-\Re \mathrm{e} \rho_{z y}^{\odot}\right]^{2} \leq\left(\rho_{z z}^{0}-\rho_{z z}^{\ominus}\right)\left(\rho_{y y}^{0}+\rho_{y y}^{\ominus}\right) . }
\end{aligned}
$$

Combining (3.104) and (3.106), (3.105) and (3.107) one gets an inequality without the inaccessible quantities $\Im \mathrm{m} \rho_{i i^{\prime}}$ :

$$
\begin{aligned}
& {\left[\Re \mathrm{e} \rho_{x y}^{\ominus}\right]^{2}+\left[\Re \mathrm{e} \rho_{x y}^{\odot}\right]^{2} \leq \rho_{y y}^{0} \rho_{x x}^{0},} \\
& {\left[\Re \mathrm{e} \rho_{y z}^{\ominus}\right]^{2}+\left[\Re \mathrm{e} \rho_{y z}^{\odot}\right]^{2} \leq \rho_{y y}^{0} \rho_{z z}^{0} .}
\end{aligned}
$$

In the notations of [52] (3.108) and (3.109) read

$$
\begin{aligned}
{\left[\rho_{1,-1}^{0}\right]^{2}+\left[\Im \mathrm{m} \rho_{1,-1}^{2}\right]^{2}+\left[\Im \mathrm{m} \rho_{1,-1}^{3}\right]^{2} } & \leq\left[\rho_{11}^{0}\right]^{2} \\
2\left[\Im \mathrm{m} \rho_{10}^{2}\right]^{2}+2\left[\Im \mathrm{m} \rho_{10}^{3}\right]^{2} & \leq \rho_{00}^{0}\left[\rho_{11}^{0}+\rho_{1,-1}^{0}\right] .
\end{aligned}
$$

The inequality (3.110) is stronger than (4) and (10) of Table 2 in [52]. The inequality (3.111) is stronger than (5) and (11) of the same table. ${ }^{14}$

The inequalities (3.108) and (3.109) are classical. For instance(3.108) can also be derived from the positivity of the meson density matrix when the photon has the polarisation $\left(S_{\oslash}, S_{\odot}, S_{\ominus}\right)=$ $(\cos \psi, \sin \psi, 0)$ with $\psi=\arg \left(\Re \mathrm{e} \rho_{x y}^{\ominus}+i \Re \mathrm{e} \rho_{x y}^{\odot}\right)$. The non-classical character of (3.104-3.107) has been lost when throwing away the information of $\Im m \rho$.

\subsubsection{Inequalities for polarised baryons}

The generalisation to the case of target polarisation, or the case where the spin of the recoiling baryon is analysed, is tedious but straightforward. Kloet et al. [20,21] have listed the observables that are accessible when the vector meson is identified through its strong-decay products.

From the expression given, e.g., by Titov et al. [137], it is possible to compute fictitious observables from randomly-generated amplitudes, and to get a first view at the allowed domain, before studying the constraints rigorously, as done for $\bar{p} p \rightarrow \bar{\Lambda} \Lambda$. However, there are twelve amplitudes, and the method becomes more time-consuming, and more delicate, as the points populate preferentially the centre of each domain, and the contour is more elusive. Some refinements are needed.

\subsubsection{Production of two pions}

A natural extension of $\pi+\mathrm{N} \rightarrow \pi+\mathrm{N}$ is the two-pion production $\pi+\mathrm{N} \rightarrow \pi+\pi+\mathrm{N}$ and, similarly, the single-pion production $\gamma+\mathrm{N} \rightarrow \pi+\mathrm{N}$ can be studied in parallel to $\gamma+\mathrm{N} \rightarrow \pi+\pi+\mathrm{N}$. This is the point of view of Ref. [64], where a rather comprehensive formalism is presented, and identities and inequalities among observables are listed.

\footnotetext{
${ }^{14}$ The $12^{\text {th }}$ inequality of this table of Ref. [52] seems erroneous
} 
Note that with three or more particles in the final states, the reaction is not planar any more. In particular, the constraints induced by parity conservation cannot be formulated as mirror symmetry with respect to the scattering plane, as discussed in Sec. 2.8.2. An exhaustive analysis of such symmetry constraints is carried out in [64]. Note, however, that all the parity constraints are classical, i.e., can be implemented directly at the level of the observables without writing down explicitly the amplitudes or the cross section matrix.

The two-pion production can also be thought as a generalisation of $\rho$ production followed by $\rho \rightarrow \pi+\pi$, without the restriction of the $\pi \pi$ system to the mass and quantum numbers of the vector meson resonance. This is the point of view adopted in most experimental analyses, with quantities measured on and off the $\rho$ peak of the $\pi \pi$ invariant mass. The aim is to distinguish the mechanism of $\rho$ production from that responsible for background $\pi \pi$ production. 


\section{Inclusive reactions}

\subsection{Spin observables in hadronic inclusive reactions}

The positivity constraints have been widely used in hadron physics to reduce the allowed domain of spin observables and, as an illustration, we will now envisage some specific examples in inclusive reactions.

\subsubsection{Total cross sections}

Let us consider the spin-dependent total cross sections for the scattering of two spin-1/2 particles

$$
a(\operatorname{spin} 1 / 2)+b(\operatorname{spin} 1 / 2) \rightarrow \text { anything },
$$

which are, for example, $\mathrm{pp}, \overline{\mathrm{p} p}, \mathrm{p} \Lambda$, pn, etc. The reaction (4.1) can be described in terms of three independent observables which are the unpolarised cross section

$$
\sigma_{\text {tot }}=\left[\sigma_{\text {tot }}(++)+\sigma_{\text {tot }}(+-)\right] / 2=\left[\sigma_{\text {tot }}(\uparrow \uparrow)+\sigma_{\text {tot }}(\uparrow \downarrow)\right] / 2
$$

and the two asymmetries

$$
\Delta \sigma_{L}=\sigma_{\text {tot }}(++)-\sigma_{\text {tot }}(+-), \quad \text { and } \quad \Delta \sigma_{T}=\sigma_{\text {tot }}(\uparrow \downarrow)-\sigma_{\text {tot }}(\uparrow \uparrow) .
$$

Here $+(-)$ denotes the longitudinally polarised (or helicity) states of $a$ and $b$ and $\uparrow(\downarrow)$ their transversely polarised states.

If $\boldsymbol{P}_{a}$ and $\boldsymbol{P}_{b}$ are the polarisation unit vectors of $a$ and $b$, the polarised total cross sections corresponding to Eq. (4.1) are [1]

$$
\sigma_{\text {tot }}\left(\boldsymbol{P}_{a}, \boldsymbol{P}_{b}\right)=\operatorname{Tr}(\mathrm{M} \rho)
$$

Here $\rho$ is the $4 \times 4$ density matrix $\rho=\left(\mathbb{1}_{2}+\boldsymbol{P}_{a} . \boldsymbol{\sigma}\right) \otimes\left(\mathbb{1}_{2}+\boldsymbol{P}_{b} . \boldsymbol{\sigma}\right) / 4$, and $\mathrm{M}$ denotes the imaginary part of the forward scattering amplitude for the elastic reaction

$$
a+b \rightarrow a+b
$$

and is the following $4 \times 4$ matrix $^{15}$

$$
\mathrm{M}=2 \sigma_{\text {tot }} \mathbb{1}_{4}-\Delta \sigma_{L} \sigma_{a z} \otimes \sigma_{b z}-\Delta \sigma_{T}\left(\sigma_{a x} \otimes \sigma_{b x}+\sigma_{a y} \otimes \sigma_{b y}\right) .
$$

$\mathrm{M}$ must be Hermitian and positive, which implies that all its principal minors, (i.e., subdeterminants of $\mathrm{M}$ with diagonal elements) must be positive, as reminded in Sec. 2.2, since $\mathrm{M}$ is a particular case of CSM. In terms of the three observables defined above, we get two trivial conditions, i.e., $\left|\Delta \sigma_{i}\right| \leq 2 \sigma_{\text {tot }},(i=L, T)$ and one non-trivial positivity bound [139], namely

$$
\left|\Delta \sigma_{T}\right| \leq \sigma_{\text {tot }}+\Delta \sigma_{L} / 2
$$

The corresponding domain is shown in Fig. 30.

In the case of proton-proton scattering, this rigorous bound is easily fulfilled for $p_{\text {lab }} \geq 1 \mathrm{GeV} / \mathrm{c}$, where $\Delta \sigma_{L}$ and $\Delta \sigma_{T}$ have been measured [140], because $\sigma_{\text {tot }}$ is rather large. For $p_{\text {lab }} \leq 1 \mathrm{GeV} / \mathrm{c}$, $\sigma_{\text {tot }}$ can be as small as $25 \mathrm{mb}$ or so and $\Delta \sigma_{L}$ can reach the value $-30 \mathrm{mb}$, so by using Eq. (4.7) one gets $\left|\Delta \sigma_{T}\right| \leq 10 \mathrm{mb}$, with some errors, an interesting limit which is, indeed, satisfied by the data $[141,142]$.

\footnotetext{
${ }^{15}$ Using the same axes $x, y, z$ for $a$ and $b$
} 


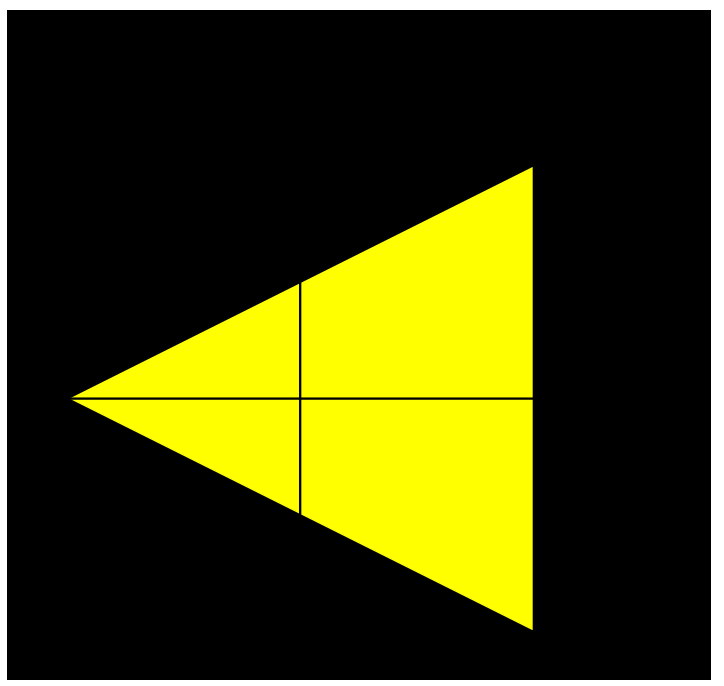

Figure 30: Domain allowed for $\Delta \sigma_{L}$ and $\Delta \sigma_{T}$

\subsubsection{Spin transfer observables}

For a parity conserving inclusive reaction of the type,

$$
\vec{a}(\text { spin } 1 / 2)+\vec{b}(\text { unpolarised }) \rightarrow c(\text { spin } 1 / 2)+X,
$$

one can define eight independent observables, which depend on three kinematic variables, $\sqrt{s}$ the centre-of-mass energy and $x_{F}, p_{T}$ the scaled longitudinal momentum and the transverse momentum of the final particle $c$. In order to define these observables, we adopt here the standard notation used in Ref. [1] $(a b \mid c X)$, where the spin direction is specified in one of the three possible directions $L, N, S$ of the Argonne convention: $\boldsymbol{L}_{a}=\boldsymbol{L}_{b}=\boldsymbol{p}_{a}, \boldsymbol{L}_{c}=\boldsymbol{p}_{c}, \boldsymbol{N}_{a}=\boldsymbol{N}_{b}=\boldsymbol{N}_{c}$ is along $\boldsymbol{p}_{a} \times \boldsymbol{p}_{c}$, and $\boldsymbol{S}_{i}=\boldsymbol{N}_{i} \times \boldsymbol{L}_{i}$. Note that $\{\boldsymbol{L}, \boldsymbol{N}, \boldsymbol{S}\}=\{\boldsymbol{l}, \boldsymbol{n} \boldsymbol{m}\}$ in the notation of Eq. (2.2) for particles $a$ and $c$ and $\{\boldsymbol{L}, \boldsymbol{N}, \boldsymbol{S}\}=\{-\boldsymbol{l}, \boldsymbol{n}-\boldsymbol{m}\}$ for particle $b$. In addition to the unpolarised cross section $\sigma_{0}$, there are seven spin dependent observables, two single transverse spin asymmetries

$$
A_{a N}=(N 0 \mid 00), \quad \text { and } \quad P_{c N}=(00 \mid N 0),
$$

and five depolarisation parameters

$$
\begin{aligned}
& D_{L L}=(L 0 \mid L 0), \quad D_{S S}=(S 0 \mid S 0), \quad D_{N N}=(N 0 \mid N 0), \\
& D_{L S}=(L 0 \mid S 0), \quad \text { and } \quad D_{S L}=(S 0 \mid L 0) \text {. }
\end{aligned}
$$

Several years ago, Doncel and Méndez [143] (see also [31]) have derived very general inequalities constraining these parameters, which read

$$
\left(1 \pm D_{N N}\right)^{2} \geq\left(A_{a N} \pm P_{c N}\right)^{2}+\left(D_{L L} \pm D_{S S}\right)^{2}+\left(D_{L S} \mp D_{S L}\right)^{2} .
$$

Let us now consider the particular reaction $\mathrm{p}^{\uparrow} \mathrm{p} \rightarrow \Lambda^{\uparrow} X$, where the incoming proton beam is polarised and the polarisation of the outgoing $\Lambda$ is measured. If we concentrate for the moment, on the case where the particle spins are normal to the scattering plane, the relevant observables are the $\Lambda$ polarisation $P_{\Lambda}$, the analysing power $A_{N}$ (denoted before $P_{c N}$ and $A_{a N}$ respectively) and the spin transfer $D_{N N}$, between the proton and the $\Lambda$. As a special case of Eq. (4.11), we obtain

$$
1 \pm D_{N N} \geq\left|P_{\Lambda} \pm A_{N}\right|,
$$

two constraints which must be satisfied for any values of the kinematic variables $\sqrt{s}, x_{F}, p_{T}$. These linear conditions are similar to those reported earlier (see Sec. 3.4) in the case of exclusive photoproduction of pseudoscalar mesons. The allowed domain corresponds to the tetrahedron shown in 
Fig. 31, which is identical to the tetrahedron encountered in $\bar{p} p \rightarrow \bar{\Lambda} \Lambda$, see Fig. 18, and in photoproduction, see Fig. 27.

These inequalities constraints involve three spin parameters, once we fixe the value of one parameter, the other two are restricted to lie in a certain domain. For instance, Fig. 31 shows also that for $D_{N N}=0, P_{\Lambda}$ and $A_{N}$ are correlated within the shaded area of a square with boundaries $(-1,+1)$. This domain is more restricted in the case $D_{N N}=1 / 3$. In the limit $D_{N N}=1$, we immediately deduce from Eqs. (4.12) that $P_{\Lambda}=A_{N}$. In the case where $D_{N N}$ is negative, one obtains the same regions, but $P_{\Lambda}$ and $A_{N}$ are interchanged with respect to their axis. In particular, for $D_{N N}=-1$ one should have $P_{\Lambda}=-A_{N}$.
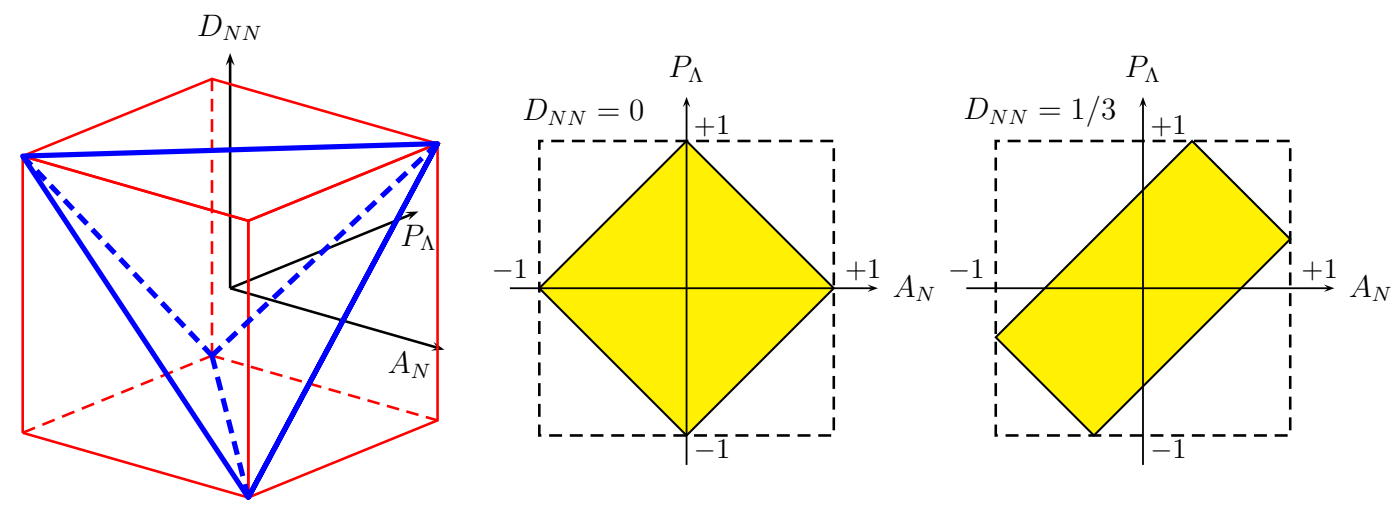

Figure 31: The allowed domain corresponding to the constraints Eq. (4.12) (left). The slice of the full domain for $D_{N N}=0($ middle $)$ and for $D_{N N}=1 / 3$ (right).

Various applications can be envisaged with the above inequalities, one concerns the analysis of experimental data and another one the validity of the spin observables predicted by theoretical models [144]. For example, at FNAL, the experiment E-704 [145-147] has performed the measurement of $P_{\Lambda}, A_{N}$ and $D_{N N}$ with transversely polarised proton beam at $200 \mathrm{GeV} / \mathrm{c}$, in the kinematic range $0.2 \leq x_{F} \leq 1.0$ and $0.1 \leq p_{T} \leq 1.5 \mathrm{GeV} / \mathrm{c}$. Their data indicate a negative $A_{N}$ and a value of $D_{N N}$ up to $30 \%$. If we take for instance $p_{T} \sim 1 \mathrm{GeV} / \mathrm{c}, x_{F} \sim 0.8$, then $D_{N N} \sim 30 \%$, for these kinematical values, they have $A_{N} \sim-10 \%$ and $P_{\Lambda} \sim-30 \%$, the inequalities (4.12) are well satisfied. These constraints can be very useful to check the experimental data and errors, if they lie outside the allow domain of positivity.

When the polarisation is not normal to the scattering plane, one has new depolarisation parameters. In the case where both $p$ and $\Lambda$ are longitudinally polarised, one can measure $D_{L L}$ and when each particle has a transverse polarisation in the scattering plane, one can measure $D_{S S}$. In this case, one gets, as a consequence of Eq. (4.11), the non-trivial, and in fact, non-classical constraints

$$
1 \pm D_{N N} \geq\left|D_{L L} \pm D_{S S}\right|, \quad 1 \pm D_{N N} \geq\left|D_{L S} \mp D_{S L}\right| .
$$

Note that the two depolarisations in the right hand side Eq. (4.13) have not yet been measured. Thus from the positivity constraints and the knowledge of some measured observables, bounds on some unknown observables are deduced.

We would like to stress the importance of new measurements which are needed to understand the spin mechanism in these reactions. The polarised pp collider at BNL-RHIC is certainly the most appropriate machine for those experiments of single and double spin measurements [32] and positivity was used to determine the allowed domain for $D_{L L}[148]$ and $D_{N N}[149,150]$. 


\subsubsection{Initial spin observables}

Let us consider an inclusive reaction of the type

$$
a(\operatorname{spin} 1 / 2)+b(\operatorname{spin} 1 / 2) \rightarrow c+X
$$

where the spins of both initial spin 1/2 particles can be in any possible directions and no polarisation is observed in the final state. Clearly, this case is related to the previous one and here we will present more details in the derivation of the results. The observables of this reaction, which are the spindependent differential cross sections with respect to the momentum of $c$, can be expressed in terms of the discontinuities (with respect to the invariant mass squared of $X$ ) of the amplitudes for the forward three-body scattering

$$
a+b+\bar{c} \rightarrow a+b+\bar{c},
$$

as given by the generalised optical theorem. With parity conservation, the knowledge of the dependence of this reaction upon the spins of $a$ and $b$ requires the determination of eight real functions, i.e., the measurement of eight independent spin observables [151]. Since the final spins are not observed, we have in fact $(a b \mid 00)$ and in addition to the unpolarised cross section $\sigma_{0}=(00 \mid 00)$, there are seven spin dependent observables, two single transverse spin asymmetries

$$
A_{a N}=(N 0 \mid 00), \quad \text { and } \quad A_{b N}=(0 N \mid 00),
$$

and five double-spin asymmetries

$$
\begin{aligned}
& A_{L L}=(L L \mid 00), \quad A_{S S}=(S S \mid 00), \quad A_{N N}=(N N \mid 00), \\
& A_{L S}=(L S \mid 00), \quad \text { and } \quad A_{S L}=(S L \mid 00) \text {, }
\end{aligned}
$$

which are in correspondence with Eqs. (4.9) and (4.10). The state of polarisation of the two spin $1 / 2$ particles $a$ and $b$ is characterised by the $2 \times 2$ density matrices $\rho_{i}=\left(\mathbb{1}_{2}+\boldsymbol{P}_{i} . \boldsymbol{\sigma}_{i}\right) / 2, i=a, b$. The state of polarisation of the incoming system in the reaction (4.14) is described by the $4 \times 4$ density matrix $\rho$, which is the direct product $\rho=\rho_{a} \otimes \rho_{b}$. The spin-dependent cross section corresponding to this reaction is

$$
\sigma\left(\boldsymbol{P}_{a}, \boldsymbol{P}_{b}\right)=\operatorname{Tr}(\mathrm{M} \rho)
$$

where $\mathcal{M}$ denotes the $4 \times 4$ cross section matrix which we shall parametrise in the following way ${ }^{16}$

$$
\begin{aligned}
\mathrm{M}=\sigma_{0}\left[\mathbb{1}_{4}+A_{a N} \sigma_{a y} \otimes \mathbb{1}_{2}+\right. & A_{b N} \mathbb{1}_{2} \otimes \sigma_{b y}+A_{N N} \sigma_{a y} \otimes \sigma_{b y}+A_{L L} \sigma_{a z} \otimes \sigma_{b z} \\
& \left.+A_{S S} \sigma_{a x} \otimes \sigma_{b x}+A_{L S} \sigma_{a z} \otimes \sigma_{b x}+A_{S L} \sigma_{a x} \otimes \sigma_{b z}\right] .
\end{aligned}
$$

Here $\sigma_{0}$ stands for the spin-averaged cross section. This expression is fully justified, since we have explicitly

$$
\begin{aligned}
\sigma\left(\boldsymbol{P}_{a}, \boldsymbol{P}_{b}\right)=\sigma_{0}\left[1+A_{a N} P_{a y}+A_{b N} P_{b y}\right. & +A_{N N} P_{a y} P_{b y}+A_{L L} P_{a z} P_{b z} \\
& \left.+A_{S S} P_{a x} P_{b x}+A_{L S} P_{a z} P_{b x}+A_{S L} P_{a x} P_{b z}\right]
\end{aligned}
$$

The crucial point is that $\mathrm{M}$ is a Hermitian and positive matrix and in order to derive the positivity conditions one should write the explicit expression of $M$ as given by Eq. (4.19). In the transversity basis, where $\sigma_{y}$ is diagonal, $\mathcal{M}$ is diagonal, and by permuting two rows and two columns, it reduces to the simple form

$$
\left(\begin{array}{c|c}
\mathrm{M}_{+} & 0 \\
\hline 0 & \mathrm{M}_{-}
\end{array}\right),
$$

\footnotetext{
${ }^{16} \mathrm{~A}$ much simpler form was used in the case of the pp total cross section, in pure spin states, to derive positivity bounds as discussed above (See Eq. (4.6)).
} 
where $\mathcal{M}_{ \pm}$are $2 \times 2$ Hermitian matrices which must be positive, leading to the following two strongest constraints $[152]^{17}$

$$
\left(1 \pm A_{N N}\right)^{2} \geq\left(A_{a N} \pm A_{b N}\right)^{2}+\left(A_{L L} \mp A_{S S}\right)^{2}+\left(A_{L S} \pm A_{S L}\right)^{2} .
$$

These inequalities are related to (4.11) by crossing, as mentioned earlier (see Sec. 2). As special cases of the above equation, we have the six weaker constraints

$$
\begin{aligned}
& 1 \pm A_{N N} \geq\left|A_{a N} \pm A_{b N}\right|, \\
& 1 \pm A_{N N} \geq\left|A_{L L} \mp A_{S S}\right|, \\
& 1 \pm A_{N N} \geq\left|A_{L S} \pm A_{S L}\right| .
\end{aligned}
$$

The first inequality is classical in the sense defined in Ref. [60] (see also Sec. 5.3): it results from the positivity of the cross section when $a$ and $b$ are polarised along $\pm \hat{y}$. The last two ones are nonclassical. They forbid a spin dependence of the form $1+\boldsymbol{P}_{a} \cdot \boldsymbol{P}_{b}$ or $1+\boldsymbol{P}_{a} \cdot R \boldsymbol{P}_{b}$, where $R$ is any rotation.

These constraints are very general and must hold in any kinematical region and for many different situations such as electron-proton scattering, electron-positron scattering or quark-quark scattering, but we now turn to a specific case, which is of direct relevance to the spin programme at the BNLRHIC polarised pp collider [32]. Now let us consider a proton-proton collision and let us call $y$ the rapidity of the outgoing particle $c$. In this case since the initial particles are identical, we have $A_{a N}(y)=-A_{b N}(-y)$ and $A_{L S}(y)=-A_{S L}(-y)$. In this case Eq. (4.22), which becomes two constraints among five independent spin observables, reads

$$
\left(1 \pm A_{N N}(y)\right)^{2} \geq\left(A_{a N}(y) \mp A_{a N}(-y)\right)^{2}+\left(A_{L L}(y) \mp A_{S S}(y)\right)^{2}+\left(A_{L S}(y) \mp A_{L S}(-y)\right)^{2} .
$$

This implies in particular, for $y=0$,

$$
1-A_{N N}(0) \geq 2\left|A_{N}(0)\right|, \quad 1-A_{N N}(0) \geq 2\left|A_{S L}(0)\right|,
$$

so that the allowed range of $A_{N}$ and $A_{S L}$ is strongly reduced if $A_{N N}$ turns out to be large and positive. Conversely if $A_{N N} \simeq-1$, these constraints are useless. Note that, in the kinematical region accessible to the pp polarised collider, a calculation of $A_{N N}$ for direct photon production and jet production has been performed [150,153]; it was found that $\left|A_{N N}\right|$ is of the order of 1 or 2 percent. Similarly, based on Ref. [154], this double transverse spin asymmetry for lepton pair production was estimated to be a few percents [155]. The direct consequence of these estimates is that $\left|A_{N}\right|$ and $\left|A_{S L}\right|$, for these processes ${ }^{18}$, are essentially bounded by $1 / 2$. In addition, from Eq. (4.23), there are two other non-trivial constraints: $1 \geq\left|A_{L L} \pm A_{S S}\right|$ when $A_{N N}$ vanishes.

Single transverse spin asymmetries in inclusive reactions at high energies are now considered to be directly related to the transverse momentum of the fundamental partons involved in the process. This new viewpoint, which has been advocated to explain the existing data in semi-inclusive deep inelastic scattering, will have to be more firmly established also by means of future data from BNLRHIC. On the theoretical side several possible leading-twist QCD mechanisms $[68,157,158]$ have been proposed to generate these asymmetries in leptoproduction [159], but also in pp collisions [160-164]. We believe that these new positivity constraints on spin observables for a wide class of reactions will be of interest for model builders as well as for future measurements.

\subsection{Spin observables in deep inelastic scattering and structure functions}

\subsubsection{Positivity constraint for the $A_{2}$ transverse asymmetry}

It is well known that Deep Inelastic Scattering (DIS), in the one-photon exchange approximation, is described as forward Compton scattering, where a very off-shell photon is probing the nucleon. In

\footnotetext{
${ }^{17}$ This article contains sign errors

${ }^{18}$ It is amusing to recall that, using a phenomenological approach for lepton-pair production, bounds on $\left|A_{N}\right|$ larger than $50 \%$ were obtained in Ref. [156], but at that time it was not known that $A_{N N}$ is small.
} 
polarised DIS one has four cross sections, $\sigma_{T}^{m}$, where $m=\left|J_{z}^{\gamma}+J_{z}^{\mathrm{N}}\right|=1 / 2$ or $3 / 2$, for photons and nucleons of definite helicities, $\sigma_{L}$ for a zero-helicity photon, and $\sigma_{L T}$ for the interference between the two cases. $L$ or $T$ indicates the longitudinal or transverse nature of the virtual photon. $\sigma_{L T}$ occurs for both the nucleon and the photon carrying transverse spin. Then one can define two asymmetries

$$
A_{1}=\frac{\sigma_{T}^{1 / 2}-\sigma_{T}^{3 / 2}}{\sigma_{T}^{1 / 2}+\sigma_{T}^{3 / 2}}, \quad \text { and } \quad A_{2}=\frac{\sigma_{L T}}{\sigma_{T}^{1 / 2}+\sigma_{T}^{3 / 2}} .
$$

There is a well-known condition established long time ago [165] and re-derived on a recent extensive study [166], written in the form

$$
\left|A_{2}\right| \leq \sqrt{R}
$$

where $R=\sigma_{L} / \sigma_{T}$ and $\sigma_{T}=\sigma_{T}^{1 / 2}+\sigma_{T}^{3 / 2}$. It reflects a non-trivial positivity condition on the photon-nucleon helicity amplitudes. We will see that it is possible to establish a bound stronger than Eq. (4.27), namely [167]

$$
\left|A_{2}\right| \leq \sqrt{R\left(1+A_{1}\right) / 2}
$$

which is also implicit from Eq. (2.32) of [166], once their notation is translated into the modern one.

Let us start with the following expressions for these four photon-nucleon cross sections in terms of the matrix elements describing the transition from the state $|H, h\rangle$ of a nucleon with helicity $H$ and a photon with helicity $h$, to the unobserved state $|X\rangle$

$$
\begin{aligned}
\sigma_{T}^{1 / 2} & =\sum_{X}|\langle+1 / 2,+1 \mid X\rangle|^{2}, \quad \sigma_{T}^{3 / 2}=\sum_{X}|\langle-1 / 2,+1 \mid X\rangle|^{2} \\
\sigma_{L} & =\sum_{X}|\langle+1 / 2,0 \mid X\rangle|^{2}=\sum_{X}|\langle-1 / 2,0 \mid X\rangle|^{2}, \\
\sigma_{L T} & =\Re \mathrm{e} \sum_{X}\langle+1 / 2,+1 \mid X\rangle\langle-1 / 2,0 \mid X\rangle^{*} .
\end{aligned}
$$

Note the different behaviour of $\sigma_{T}^{\{1,3\} / 2}, \sigma_{L}$ and $\sigma_{L T}$ when one or two helicities flip sign, due to symmetries and conservation of angular momentum. We are now ready to write down the CauchySchwarz inequality as

$$
\sum_{X}|\langle+1 / 2,+1 \mid X\rangle \pm a\langle-1 / 2,0 \mid X\rangle|^{2} \geq 0
$$

where $a$ is a positive real number. By making use of the definitions (4.29) and after the standard minimisation with respect to the choice of $a$, one immediately arrives at

$$
\left|\sigma_{L T}\right| \leq \sqrt{\sigma_{L} \sigma_{T}^{1 / 2}}
$$

leading directly to Eq. (4.28). The value of this new bound is clearly related to the size of $A_{1}$ and it becomes very useful for negative $A_{1}$, like in the neutron case.

One may apply this method for each quark flavour separately. Decomposing the cross-sections as

$$
\sigma_{i}=\sum_{f} e_{i}^{2} \sigma_{i}^{f} \quad(i=L, T, L T)
$$

positivity implies that

$$
\left|\sigma_{L T}^{f}\right| \leq \sqrt{\sigma_{L}^{f} \sigma_{T}^{1 / 2, f}}, \quad\left|A_{2}^{f}\right| \leq \sqrt{R^{f}\left(1+A_{1}^{f}\right) / 2} .
$$

Some consequences of these positivity constraints in connection with higher twists contributions have been discussed in Ref. [167]. 


\subsubsection{Positivity Constraints for Structure Functions}

In the previous Sec. 4.2.1, we derived constraints for DIS observables related to the photon-nucleon forward helicity amplitudes ${ }^{19}$. However, DIS is fully described by the hadronic tensor, so the positivity constraints in DIS may be also derived from the positivity properties of this tensor. We will present their derivation in the most general case, when the nucleon is polarised and parity violation $(\mathrm{PV})$ is taken into account.

In PV lepton-nucleon DIS all the observables arise form the contraction

$$
\ell^{\mu \nu} W_{\mu \nu}
$$

which involves the leptonic tensor $\ell^{\mu \nu}$ and the hadronic tensor $W_{\mu \nu}(p, \mathrm{~s}, q)$ of the nucleon, where $p$ and $\mathrm{s}$ are the energy-momentum and polarisation vectors of the nucleon, respectively $\left(p^{2}=\right.$ $M^{2}, \mathrm{~s}^{2}=-1$ and $p . \mathrm{s}=0$ ); $k$ and $k^{\prime}$ are the four momenta of the initial and final leptons, respectively, and $q=k-k^{\prime}$ is the momentum transfer. Using Lorentz invariance and time reversal invariance, one can express $W_{\mu \nu}(p, \mathrm{~s}, q)$ in terms of 14 real structure functions as follows [169] ${ }^{20}$,

$$
\begin{aligned}
W_{\mu \nu}(p, \mathrm{~s}, q)= & -g_{\mu \nu}\left(W_{1}\left(\nu, q^{2}\right)+X_{1}\left(\nu, q^{2}\right) \frac{\mathrm{s} . q}{M}\right) \\
& +\frac{p_{\mu} p_{\nu}}{M^{2}}\left(W_{2}\left(\nu, q^{2}\right)+X_{2}\left(\nu, q^{2}\right) \frac{\mathrm{s} . q}{M}\right) r \\
& -i \epsilon_{\mu \nu \alpha \beta}\left[\frac{p^{\alpha}}{2 M^{2}}\left(q^{\beta} W_{3}\left(\nu, q^{2}\right)-2 M \mathrm{~s}^{\beta} G_{3}\left(\nu, q^{2}\right)\right)\right. \\
& \left.+\frac{q^{\alpha}}{M}\left(\mathrm{~s}^{\beta} G_{1}\left(\nu, q^{2}\right)+\left(\nu \mathrm{s}^{\beta}-\frac{(\mathrm{s} . q)}{M} p^{\beta}\right) \frac{G_{2}}{M}\left(\nu, q^{2}\right)\right)\right] \\
& +\frac{q_{\mu} q_{\nu}}{M^{2}}\left(W_{4}\left(\nu, q^{2}\right)+X_{4}\left(\nu, q^{2}\right) \frac{\mathrm{s} \cdot q}{M}\right) \\
& +\frac{p_{\mu} q_{\nu}+q_{\mu} p_{\nu}}{2 M^{2}}\left(W_{5}\left(\nu, q^{2}\right)+X_{5}\left(\nu, q^{2}\right) \frac{\mathrm{s} . q}{M}\right) \\
& +\frac{p_{\mu} \mathrm{s}_{\nu}+\mathrm{s}_{\mu} p_{\nu}}{2 M} Z_{1}\left(\nu, q^{2}\right)+\frac{q_{\mu} \mathrm{s}_{\nu}+\mathrm{s}_{\mu} q_{\nu}}{2 M} Z_{2}\left(\nu, q^{2}\right) .
\end{aligned}
$$

Here $\epsilon_{\mu \nu \alpha \beta}$ is the total antisymmetric tensor with $\epsilon_{0123}=+1$. All structure functions, which are made dimensionless by including appropriate mass factors, depend on two Lorentz scalars, $\nu=$ $p . q / M$ and $Q^{2}=-q^{2}>0$, where $M$ is the nucleon mass. Seven of them, $W_{1}, W_{2}, W_{4}, W_{5}, G_{1}$, $G_{2}$ and $G_{3}$ appear in parity-conserving processes and the other seven $W_{3}, X_{1}, X_{2}, X_{3}, X_{4}, Z_{1}$ and $Z_{2}$, are related to PV processes involving weak interactions. Note that only the $W_{i}$ 's are independent of the polarisation vector $s$ and they characterise the scattering of an unpolarised nucleon.

Clearly the hadronic tensor $W_{\mu \nu}(p, \mathbf{s}, q)$ is Hermitian

$$
W_{\mu \nu}(p, \mathrm{~s}, q)=W_{\nu \mu}^{*}(p, \mathrm{~s}, q),
$$

and semi-positive. This last property follows from its definition as a bilinear expression of quarks currents and implies that

$$
a_{\mu}^{*} W_{\mu \nu}(p, \mathrm{~s}, q) a_{\nu} \geq 0,
$$

for any complex 4-vector $a_{\mu}$. The resulting constraints are valid for the most general case of PV and spin-dependent scattering, mentioned above. Moreover, they can be generalised to Semi-Inclusive Deep Inelastic Scattering (SIDIS), which involves 18 structure functions, if parity is conserved [170, 171], and they may also be applied to the Drell-Yan process, related to it by crossing.

Let us now further discuss the complexity arising when the nucleon target polarisation is taken into account. Following Refs. [166,170], one should consider instead of Eq. (4.37), the more general condition

$$
\sum_{\lambda . \lambda^{\prime}} a_{\mu, \lambda}^{*} W_{\mu \nu}\left(p, q, \lambda, \lambda^{\prime}\right) a_{\nu, \lambda^{\prime}} \geq 0
$$

\footnotetext{
${ }^{19}$ Positivity constraints for non-forward Compton amplitudes were derived in Ref. [168].

${ }^{20}$ This expression slightly differs from Eq. (3) of Ref. [169].
} 
in 8-dimensional space including, besides Lorentz indices, the spinor indices $\lambda$ and $\lambda^{\prime} . a_{\mu \lambda}$ is a compound photon-nucleon spin wave function, which might be entangled ${ }^{21}$. In particular, the inequalities (4.28) and (4.33) on $A_{2}$ require entangled states. The expressions resulting from (4.38) can be very complicated and we will restrict ourselves to the positivity constraints in the case of longitudinal polarisation of degree $\xi$, so we have $\mathrm{s}=(0, \xi, 0,0)$. In that case $W_{\mu \nu}(p, \mathrm{~s}, q)$ in the laboratory frame has the block-diagonal form

$$
\left(\begin{array}{cc}
N_{1} & 0 \\
0 & N_{0}
\end{array}\right), \quad N_{\alpha}=M_{\alpha}+\xi \Delta M_{\alpha}=\left(\begin{array}{cc}
M_{\alpha, 11}+\xi \Delta M_{\alpha, 11} & M_{\alpha, 12}+\xi \Delta M_{\alpha, 12} \\
M_{\alpha, 12}^{*}+\xi \Delta M_{\alpha, 12}^{*} & M_{\alpha, 22}+\xi \Delta M_{\alpha, 22}
\end{array}\right),
$$

where $\alpha=0,1$ and $M_{\alpha, i j}$ (resp. $\Delta M_{\alpha, i j}$ ) stands for the $i j$ matrix element of $M_{\alpha}$ (resp. $\Delta M_{\alpha}$ ). The target spin-averaged part is described by $M_{1}$ and $M_{0}$ which are the following $2 \times 2$ Hermitian matrices

$$
M_{1}=\left(\begin{array}{cc}
-W_{1}+W_{2}+\nu^{\prime 2} W_{4}+\nu^{\prime} W_{5} & Q^{\prime}\left(\nu^{\prime} W_{4}+W_{5} / 2\right) \\
Q^{\prime}\left(\nu^{\prime} W_{4}+W_{5} / 2\right) & W_{1}+Q^{2} W_{4}
\end{array}\right),
$$

and

$$
M_{0}=\left(\begin{array}{cc}
W_{1} & -i Q^{\prime} W_{3} / 2 \\
+i Q^{\prime} W_{3} / 2 & W_{1}
\end{array}\right)
$$

where $\nu^{\prime}=\nu / M$ and $Q^{\prime}=\sqrt{\nu^{2}+Q^{2}} / M$. The dependence on target polarisation is described by two similar $2 \times 2$ Hermitian matrices $\Delta M_{1}$ and $\Delta M_{0}$ with the following entries:

$$
\begin{aligned}
& \Delta M_{1,11}=Q^{\prime}\left(X_{2}-X_{1}+\nu^{\prime 2} X_{4}+\nu^{\prime} X_{5}\right), \\
& \Delta M_{1,12}=Z_{1} / 2+\nu^{\prime} Z_{2} / 2+Q^{\prime 2}\left(X_{5}+\nu^{\prime} X_{4}\right), \\
& \Delta M_{1,22}=Q^{\prime}\left(-X_{1}+Q^{\prime 2} X_{4}+Z_{2}\right), \\
& \Delta M_{0,11}=\Delta M_{0,22}=Q^{\prime} X_{1}, \\
& \Delta M_{0,12}=-i\left(G_{3}+\nu^{\prime} G_{1}-Q^{2} G_{2} / M^{2}\right) .
\end{aligned}
$$

Note that $N_{0}$ is diagonal in the circular-polarisation basis, because of the conservation of $J_{z}$.

The necessary and sufficient conditions for $W_{\mu \nu}(p, \mathrm{~s}, q)$ to satisfy the inequality (4.37) are, according to Sec. 2.2, that all the principal minors of $N_{1}$ and $N_{0}$ should be positive definite for any $|\xi|<1$. For the diagonal elements, since two inequalities for the matrix $N_{0}$ coincide, we have three inequalities linear in the $W_{i}$

$$
M_{\alpha, i i}-\left|\Delta M_{\alpha, i i}\right| \geq 0,
$$

where $i=1,2$. The positivity of the $2 \times 2$ determinants of $N_{0}$ and $N_{1}$ reads

$$
a_{\alpha}+\left(b_{\alpha}^{(1)}+b_{\alpha}^{(2)}\right) \xi+c_{\alpha} \xi^{2} \geq 0,
$$

where

$$
\begin{aligned}
a_{\alpha} & =\operatorname{det}\left(M_{\alpha}\right), & c_{\alpha} & =\operatorname{det}\left(\Delta M_{\alpha}\right), \\
b_{\alpha}^{(1)} & =\left|\begin{array}{cc}
M_{\alpha, 11} & M_{\alpha, 12} \\
\Delta M_{\alpha, 12}^{*} & \Delta M_{\alpha, 22}
\end{array}\right|, & b_{\alpha}^{(2)} & =\left|\begin{array}{cc}
\Delta M_{\alpha, 11} & \Delta M_{\alpha, 12} \\
M_{\alpha, 12}^{*} & M_{\alpha, 22}^{*}
\end{array}\right| .
\end{aligned}
$$

Due to convexity, it is sufficient that (4.44) is satisfied for $\xi=+1$ and $\xi=-1$. This gives the following inequalities quadratic in the $W_{i}$ 's

$$
\left|b_{\alpha}^{(1)}+b_{\alpha}^{(2)}\right| \leq a_{\alpha}+c_{\alpha} .
$$

Note that $a_{\alpha}$ is positive because of the positivity in the spin-averaged case $\xi=0$. The condition $\left|c_{\alpha}\right| \leq a_{\alpha}$ follows from Eq. (4.43). This is most conveniently seen by performing a unitary transformation such that $M_{\alpha}$ becomes diagonal. As the moduli of diagonal entries of $\Delta M_{\alpha}$ cannot exceed those of $M_{\alpha}, \operatorname{det}\left(\Delta M_{\alpha}\right) \leq \operatorname{det}\left(M_{\alpha}\right)$.

\footnotetext{
${ }^{21} W_{\mu \nu}\left(p, q, \lambda, \lambda^{\prime}\right)$ is a particular type of cross section matrix which could be written $\mathcal{R}_{\mu, \lambda, \nu, \lambda^{\prime}}$ in the notation of Sec. 2.
} 


\subsubsection{Neutrino Deep Inelastic Scattering}

We are now turning to an application ${ }^{22}$ of positivity inequalities for neutrino deep inelastic scattering [174]. We consider the case of unpolarised nucleons which is technically simpler and is of more practical importance. We keep the possibility of the detecting the polarisation of the final-state lepton, to better exhibit the power of positivity constraints. The corresponding application concerns the polarisation of a produced $\tau$ lepton which is of major importance for neutrino physics.

We should now consider the inequalities of Sec. 4.2.2 in the special case $\xi=0$. So for the diagonal elements we have from Eq. (4.43) the following three inequalities linear in the $W_{i}^{\prime}$ s:

$$
\begin{aligned}
W_{1} & \geq 0, \\
-W_{1}+W_{2}+\frac{\nu^{2}}{M^{2}} W_{4}+\frac{\nu}{M} W_{5} & \geq 0, \\
W_{1}+\frac{\nu^{2}+Q^{2}}{M^{2}} W_{4} & \geq 0 .
\end{aligned}
$$

The quadratic inequalities following from from the $2 \times 2$ determinants of $M_{0}$ and $M_{1}$ are of the form:

$$
W_{1}^{2} \geq \frac{\nu^{2}+Q^{2}}{4 M^{2}} W_{3}^{2}, \quad \text { or equivalently } \quad W_{1} \geq \frac{\sqrt{\nu^{2}+Q^{2}}}{2 M}\left|W_{3}\right|,
$$

and

$$
\left(-W_{1}+W_{2}+\frac{\nu^{2}}{M^{2}} W_{4}+\frac{\nu}{M} W_{5}\right)\left(W_{1}+\frac{\nu^{2}+Q^{2}}{M^{2}} W_{4}\right) \geq \frac{\nu^{2}+Q^{2}}{M^{2}}\left(\frac{\nu}{M} W_{4}+\frac{1}{2} W_{5}\right)^{2} .
$$

By imposing the last condition, only one of the two inequalities Eq. (4.48) or Eq. (4.49) is needed, which is a consequence of the existence of no more than 4 independent inequalities as discussed in Sec. 2.3.

Let us pass to the applications of the inequalities. In the laboratory frame, let us denote by $E_{\nu}$, $E_{\tau}$ and $p_{\tau}$ the neutrino energy, $\tau$ energy and momentum, respectively and $\theta$ the scattering angle. We then have $\nu=E_{\nu}-E_{\tau}$ and $Q^{2}=2 E_{\nu}\left[E_{\tau}-p_{\tau} \cos \theta\right]-m_{\tau}^{2}$, where $m_{\tau}=1.777 \mathrm{GeV}$ is the $\tau$ mass. Finally, the Bjorken variable $x$ is defined as usual: $x=Q^{2} / 2 p . q$ and the physical region is $x_{\min } \leq x \leq 1$, where $x_{\min }=m_{\tau}^{2} / 2 M\left(E_{\nu}-m_{\tau}\right)$. The unpolarised cross sections for deep inelastic scattering are expressed as

$$
\frac{\mathrm{d} \sigma^{ \pm}}{\mathrm{d} E_{\tau} \mathrm{d} \cos \theta}=\frac{G_{F}^{2}}{2 \pi} \frac{M_{W}^{4} p_{\tau}}{\left(Q^{2}+M_{W}^{2}\right)^{2}} R_{ \pm}
$$

where $G_{F}$ is the Fermi constant and $M_{W}$ is the $W$-boson mass. Here

$$
\begin{aligned}
R_{ \pm}=\frac{1}{M}\left\{\left(2 W_{1}+\frac{m_{\tau}^{2}}{M^{2}} W_{4}\right)\right. & \left(E_{\tau}-p_{\tau} \cos \theta\right)+W_{2}\left(E_{\tau}+p_{\tau} \cos \theta\right) \\
\pm & \left.\frac{W_{3}}{M}\left(E_{\nu} E_{\tau}+p_{\tau}^{2}-\left(E_{\nu}+E_{\tau}\right) p_{\tau} \cos \theta\right)-\frac{m_{\tau}^{2}}{M} W_{5}\right\}
\end{aligned}
$$

where the \pm signs correspond to $\tau^{\mp}$ productions.

Because of time reversal invariance, the polarisation vector $\vec{P}$ of the $\tau$ in its rest frame, lies in the scattering plane defined by the momenta of the incident neutrino and the produced $\tau$. It has a component $P_{L}$ along the direction of $\overrightarrow{p_{\tau}}$ and a component $P_{P}$ perpendicular to $\overrightarrow{p_{\tau}}$, whose expressions

\footnotetext{
${ }^{22}$ For other applications to DIS or SIDIS, see Refs. [172,173].
} 
are, in the laboratory frame,

$$
\begin{aligned}
P_{P}=\mp \frac{m_{\tau} \sin \theta}{M R_{ \pm}}\left(2 W_{1}-W_{2} \pm \frac{E_{\nu}}{M} W_{3}-\frac{m_{\tau}^{2}}{M^{2}} W_{4}+\frac{E_{\tau}}{M} W_{5}\right) \\
P_{L}=\mp \frac{1}{M R_{ \pm}}\left\{\left(2 W_{1}-\frac{m_{\tau}^{2}}{M^{2}} W_{4}\right)\left(p_{\tau}-E_{\tau} \cos \theta\right)+W_{2}\left(p_{\tau}+E_{\tau} \cos \theta\right)\right. \\
\left. \pm \frac{W_{3}}{M}\left(\left(E_{\nu}+E_{\tau}\right) p_{\tau}-\left(E_{\nu} E_{\tau}+p_{\tau}^{2}\right) \cos \theta\right)-\frac{m_{\tau}^{2}}{M} W_{5} \cos \theta\right\} .
\end{aligned}
$$

In addition, it is convenient to introduce also the degree of polarisation defined as $P=\sqrt{P_{P}^{2}+P_{L}^{2}}$. As previously the \pm signs correspond to $\tau^{\mp}$ productions and it is clear that if $W_{3}=0$, one has $R_{+}=R_{-}$and $\tau^{+}$and $\tau^{-}$have opposite polarisations.

In order to test the usefulness of positivity constraints to restrict the allowed domains for $P_{P}$ and $P_{L}$, we proceed by the following method, similar to that of Sec. 3.3, without referring to a specific model for the $W_{i}$ 's. We generate randomly the values of the $W_{i}$ 's, in the range $[0,+1]$ for $W_{1}$ and $W_{2}$, which are clearly positive and $[-1,+1]$ for $i=3,4,5$. The most trivial positivity constraints are $R_{ \pm} \geq 0$, but in fact they are too weak and do not imply the obvious requirements $\left|P_{L}\right| \leq 1$ and $\left|P_{P}\right| \leq 1$ or $P \leq 1^{23}$. So we first impose $R_{ \pm} \geq 0$ and $P \leq 1$ for different values of $E_{\nu}$, $Q^{2}$ and $x$ and as shown in Fig. 32, for $\tau^{+}$production, the points which satisfy these constraints are represented by grey dots inside the disk, $P_{L}^{2}+P_{P}^{2} \leq 1$. If we now add the non trivial positivity constraints Eqs. (4.47-4.51), which also guarantee that $P \leq 1$, we get the black dots, giving a much smaller area. In Fig. 32, the top row corresponds to $E_{\nu}=10 \mathrm{GeV}$ and $Q^{2}=1 \mathrm{GeV}^{2}$, the row below to $E_{\nu}=10 \mathrm{GeV}$ and $Q^{2}=4 \mathrm{GeV}^{2}$ and the next two rows to $E_{\nu}=20 \mathrm{GeV}$ and $Q^{2}=1$ or $4 \mathrm{GeV}^{2}$. Going from left to right $x$ increases from a value close to its minimum to 0.9 . It is interesting to note that the black allowed area increases with $Q^{2}$ and becomes smaller for increasing incident energy and increasing $x$. For $\tau^{-}$production, the corresponding areas are obtained by symmetry with respect to the centre of the disk. For increasing $x$, since $P_{L}$ is more and more restricted to values close to +1 for $\tau^{+}$( -1 for $\tau^{-}$), it is striking to observe that the non trivial positivity constraints lead to a situation where the $\tau^{+}\left(\tau^{-}\right)$is almost purely right-handed (left-handed), although it has a non zero mass.

Another way to present our results is seen in Fig. 33, which shows the upper and lower bounds from the non trivial positivity constraints for a given incident energy and different $x$ values, versus $Q^{2}$. These bounds are obtained by selecting the larger and smaller allowed values of $P_{L}$ and $P_{P}$, when the $W_{i}$ 's are varied for a fixed bin of $E_{\nu}$ and $x$. We also indicate the scattering angle which increases with $Q^{2}$ and we recall that for $\theta=0$ we have $P_{P}=0$ (see Eq. (4.54)).

Finally, as an example for a particular kinematic situation we show in Fig. 34 the effect of imposing the Callan-Gross relation [175], namely $Q^{2} W_{1}=\nu^{2} W_{2}$. It further reduces both the grey dots and the black dots areas, since this has to be compared with the first row of Fig. 32. For the same kinematic situation we also show in Fig. 34, the effect of the Albright-Jarlskog relations [176], namely $M W_{1}=\nu W_{5}$ and $W_{4}=0$, and we observe again that the allowed regions are much smaller. This example illustrates the fact that a more precise knowledge of the structure functions $W_{i}$ 's, will certainly further restrict the domains shown in Fig. 32.

\subsubsection{Photon structure functions}

Let us now investigate the model-independent constraints for the structure functions of virtual (offshell) and real (on-shell) photon target. In Refs. [177, 178], three positivity conditions for the virtual photon case and one condition for the real photon were derived, the latter of which relates the polarised and unpolarised structure functions.

\footnotetext{
${ }^{23}$ Note that in the trivial case where $W_{3}=W_{4}=W_{5}=0, R \geq 0$ implies $P \leq 1$.
} 

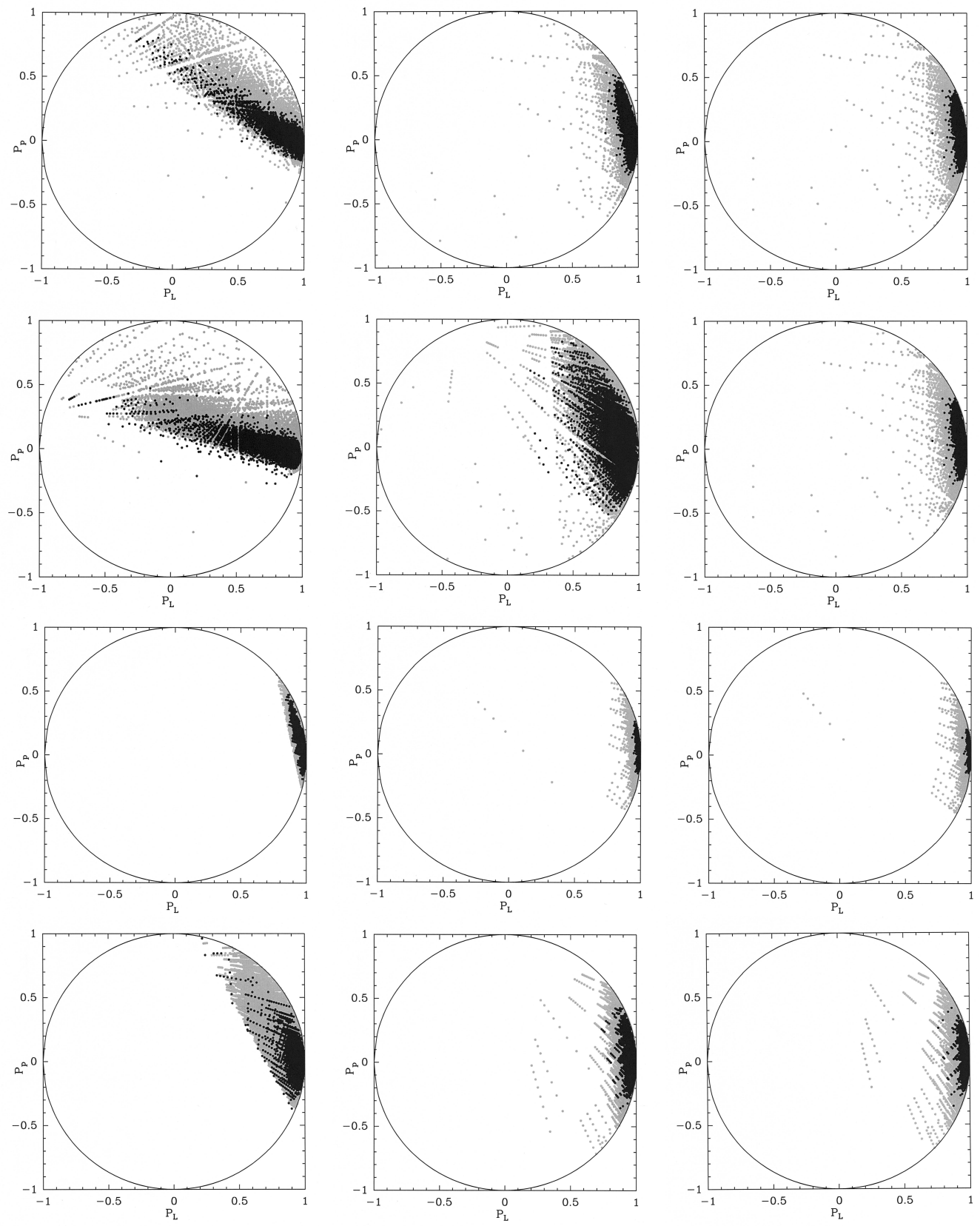

Figure 32: For $\tau^{+}$production, $P_{P}$ versus $P_{L}$ in a domain limited by $R_{+} \geq 0, P \leq 1$ (grey dots inside disk) plus non trivial positivity constraints (black dots inside disk). From top to bottom and left to right, $E_{\nu}=10 \mathrm{GeV}, Q^{2}=1 \mathrm{GeV}^{2}, x=0.25,0.6,0.9, E_{\nu}=10 \mathrm{GeV}, Q^{2}=4 \mathrm{GeV}^{2}, x=$ $0.4,0.6,0.9, E_{\nu}=20 \mathrm{GeV}, Q^{2}=1 \mathrm{GeV}^{2}, x=0.25,0.6,0.9, E_{\nu}=20 \mathrm{GeV}, Q^{2}=4 \mathrm{GeV}^{2}, x=$ $0.25,0.6,0.9$.

Consider the virtual photon-photon forward scattering $\gamma(q)+\gamma(p) \rightarrow \gamma(q)+\gamma(p)$ illustrated in Fig. 35. The $s$-channel helicity amplitudes are given by

$$
W\left(a, b \mid a^{\prime}, b^{\prime}\right)=\epsilon_{\mu}^{*}(a) \epsilon_{\rho}^{*}(b) W^{\mu \nu \rho \tau} \epsilon_{\nu}\left(a^{\prime}\right) \epsilon_{\tau}\left(b^{\prime}\right),
$$



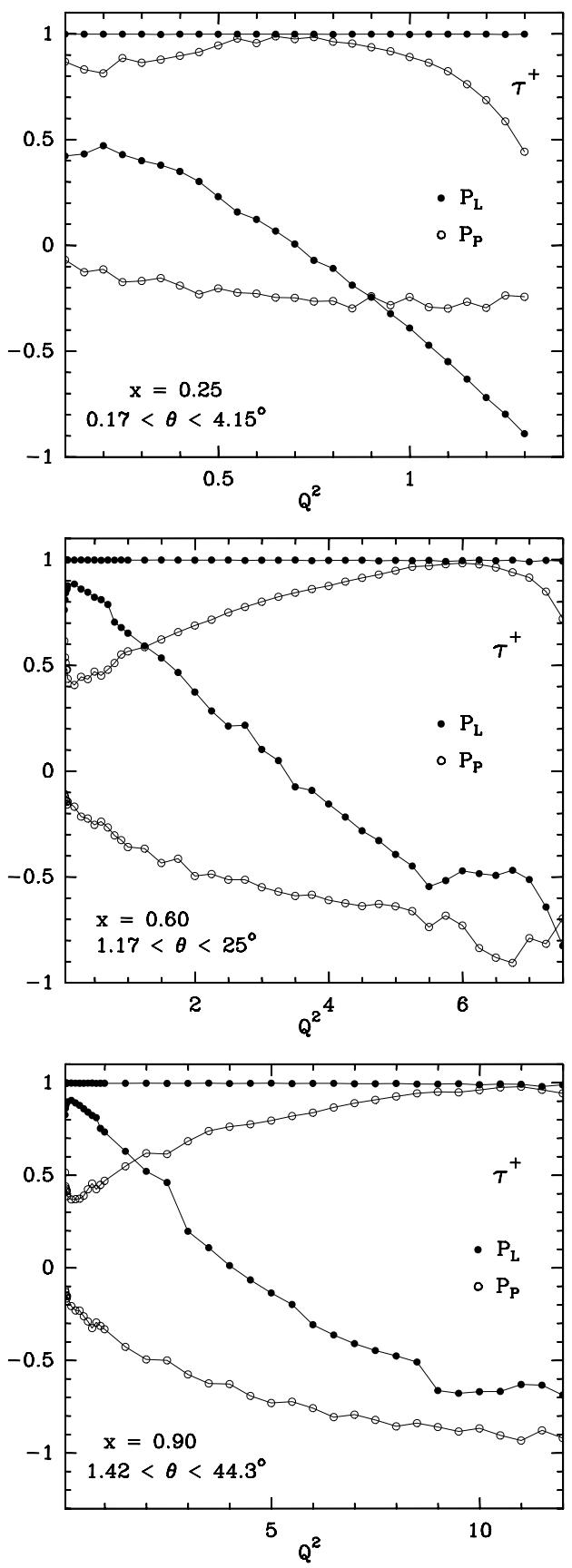

Figure 33: For $\tau^{+}$production, upper and lower bounds on $P_{P}$ (open circles) and $P_{L}$ (full circles) as a function of $Q^{2}$ for $E_{\nu}=10 \mathrm{GeV}$ and $x=$ $0.25,0.6,0.9$.

where $p$ and $q$ are the four-momenta of the incoming and outgoing photons and $\epsilon_{\mu}(a)$ represents the photon polarisation vector with helicity $a=0, \pm 1$. Similarly for the other polarisation vectors we have and $a^{\prime}, b, b^{\prime}=0, \pm 1$. Due to the angular momentum conservation, $W\left(a, b \mid a^{\prime}, b^{\prime}\right)$ vanishes unless it satisfies the condition $a-b=a^{\prime}-b^{\prime}$. Parity conservation and time reversal invariance lead to the following properties for $W\left(a, b \mid a^{\prime}, b^{\prime}\right)[1]$ :

$$
\begin{aligned}
& W\left(a, b \mid a^{\prime}, b^{\prime}\right)=W\left(-a,-b \mid-a^{\prime},-b^{\prime}\right) \quad \text { Parity conservation , } \\
& W\left(a, b \mid a^{\prime}, b^{\prime}\right)=W\left(a^{\prime}, b^{\prime} \mid a, b\right) \quad \text { Time reversal invariance . }
\end{aligned}
$$

Thus in total we have eight independent $s$-channel helicity amplitudes, which we may take as 

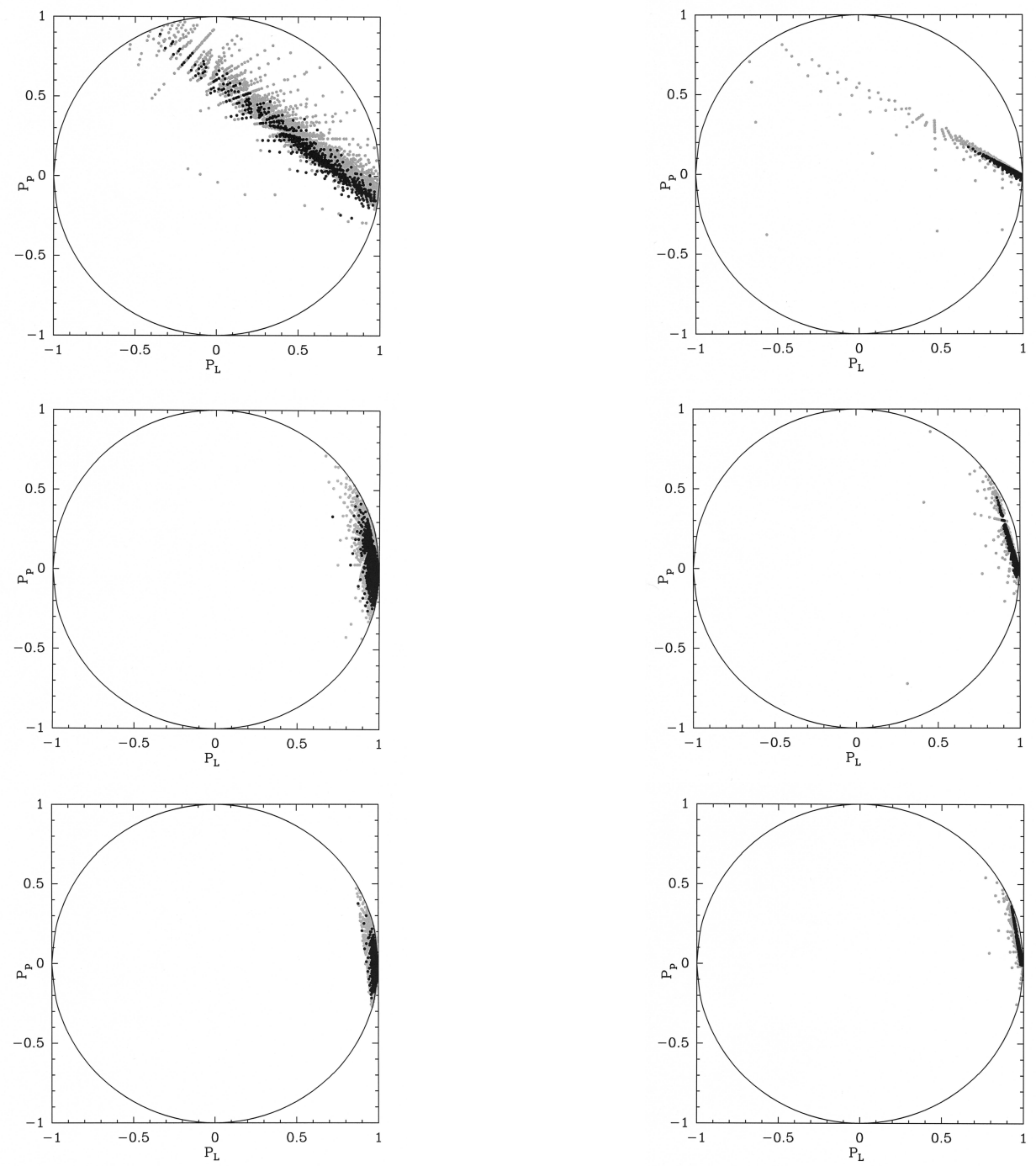

Figure 34: For $\tau^{+}$production, $P_{P}$ versus $P_{L}$ in a domain limited by $R_{+} \geq 0, P \leq 1$ assuming the Callan-Gross (left) or the Albright-Jarlskog (right) relations (grey dots inside disk) plus non trivial positivity constraints (black dots inside disk). $E_{\nu}=10 \mathrm{GeV}, Q^{2}=1 \mathrm{GeV}^{2}$, from top to bottom, $x=0.25,0.6,0.9$.

$W(1,1 \mid 1,1), \quad W(1,-1 \mid 1,-1), \quad W(1,0 \mid 1,0), \quad W(0,1 \mid 0,1), \quad W(0,0 \mid 0,0), \quad W(1,1 \mid-1,-1)$, $W(1,1 \mid 0,0)$ and $W(1,0 \mid 0,-1)$. The first five amplitudes are helicity-nonflip and the rest are helicity-flip. We note that $s$-channel helicity-nonflip amplitudes are semi-positive, but not the helicity-flip ones. Moreover corresponding to these three helicity-flip amplitudes, we will obtain three non-trivial positivity constraints.

The helicity amplitudes may be expressed in terms of the transition matrix elements from the 


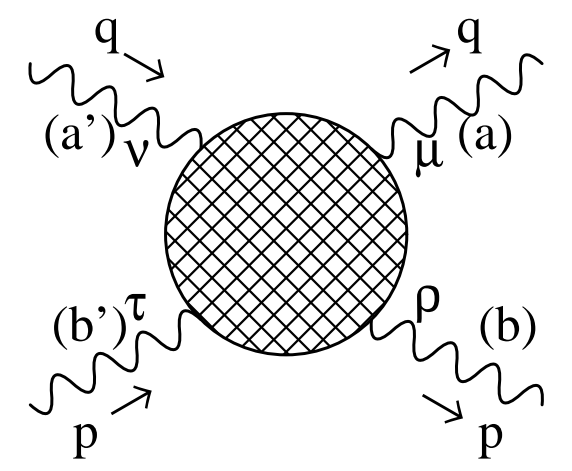

Figure 35: Virtual photon-photon forward scattering with momenta $q(p)$ and helicities $a(b)$ and $a^{\prime}\left(b^{\prime}\right)$.

state $|a, b\rangle$ of two virtual photons with helicities $a$ and $b$, to the unobserved state $|X\rangle$ as

$$
\begin{aligned}
W(a, b \mid a, b) & =\sum_{X}|\langle X \mid a, b\rangle|^{2}, \\
W\left(a, b \mid a^{\prime}, b^{\prime}\right) & =\Re \mathrm{e} \sum_{X}\langle X \mid a, b\rangle^{*}\left\langle X \mid a^{\prime}, b^{\prime}\right\rangle \quad \text { for } \quad\left(a \neq a^{\prime}, b \neq b^{\prime}\right) .
\end{aligned}
$$

Then, a Cauchy-Schwarz inequality $[154,167]$

$$
\sum_{X}\left|\langle X \mid a, b\rangle+\alpha\left\langle X \mid a^{\prime}, b^{\prime}\right\rangle\right|^{2} \geq 0
$$

which holds for an arbitrary real number $\alpha$, leads to a positivity bound for the helicity amplitudes, i.e., $\left|W\left(a, b \mid a^{\prime}, b^{\prime}\right)\right| \leq \sqrt{W(a, b \mid a, b) W\left(a^{\prime}, b^{\prime} \mid a^{\prime}, b^{\prime}\right)}$. Writing down explicitly, we obtain the following three positivity constraints:

$$
\begin{aligned}
|W(1,1 \mid-1,-1)| & \leq W(1,1 \mid 1,1), \\
|W(1,1 \mid 0,0)| & \leq \sqrt{W(1,1 \mid 1,1) W(0,0 \mid 0,0)} \\
|W(1,0 \mid 0,-1)| & \leq \sqrt{W(1,0 \mid 1,0) W(0,1 \mid 0,1)} .
\end{aligned}
$$

In terms of the eight independent amplitudes introduced by Budnev, Chernyak and Ginzburg [179], the $s$-channel helicity amplitudes are related as follows

$$
\begin{aligned}
W_{T T}^{\tau} & =W(1,1 \mid-1,-1), & W_{T T} & =\frac{1}{2}[W(1,1 \mid 1,1)+W(1,-1 \mid 1,-1)], \\
W_{S T} & =W(0,1 \mid 0,1), & W_{T T}^{a} & =\frac{1}{2}[W(1,1 \mid 1,1)-W(1,-1 \mid 1,-1)], \\
W_{T S} & =W(1,0 \mid 1,0), & W_{T S}^{\tau} & =\frac{1}{2}[W(1,1 \mid 0,0)-W(1,0 \mid 0,-1)], \\
W_{S S} & =W(0,0 \mid 0,0), & W_{T S}^{\tau a} & =\frac{1}{2}[W(1,1 \mid 0,0)+W(1,0 \mid 0,-1)] .
\end{aligned}
$$

Since the helicity-nonflip amplitudes are non-negative, the first four structure functions are positive definite and the last four are not. 
The above three conditions Eqs. (4.59) can be rewritten as

$$
\begin{aligned}
\left|W_{\mathrm{TT}}^{\tau}\right| & \leq\left(W_{\mathrm{TT}}+W_{\mathrm{TT}}^{a}\right), \\
\left|W_{\mathrm{TS}}^{\tau}+W_{\mathrm{TS}}^{\tau a}\right| & \leq \sqrt{\left(W_{\mathrm{TT}}+W_{\mathrm{TT}}^{a}\right) W_{\mathrm{SS}}}, \\
\left|W_{\mathrm{TS}}^{\tau}-W_{\mathrm{TS}}^{\tau a}\right| & \leq \sqrt{W_{\mathrm{TS}} W_{\mathrm{ST}}},
\end{aligned}
$$

where $\mathrm{T}$ and $\mathrm{S}$ refer to the transverse and longitudinal photon, respectively, and the superscripts " $\tau$ " and " $a$ " imply the relevance to the helicity-flip amplitudes and polarised ones, respectively. For the real photon, $p^{2}=0$, the number of independent helicity amplitudes reduces to four. They are $W(1,1 \mid 1,1), W(1,-1 \mid 1,-1), W(0,1 \mid 0,1)$, and $W(1,1 \mid-1,-1)$, which are related to four structure functions $W_{i}^{\gamma}$ as follows [180]:

$$
\begin{aligned}
& W(1,1 \mid 1,1)+W(1,-1 \mid 1,-1)=2 W_{1}^{\gamma}, \\
& W(0,1 \mid 0,1)=-W_{1}^{\gamma}+(p . q)^{2} W_{2}^{\gamma} / Q^{2}, \\
& W(1,1 \mid-1,-1)=2 W_{3}^{\gamma}, \\
& W(1,1 \mid 1,1)-W(1,-1 \mid 1,-1)=2 W_{4}^{\gamma},
\end{aligned}
$$

where the last one is the polarised structure function and usually denoted by $g_{1}^{\gamma}$ with $W_{4}^{\gamma}=g_{1}^{\gamma} / 2$. Also the first one, $W_{1}^{\gamma}$, is often referred to as $F_{1}^{\gamma}$ with $W_{1}^{\gamma}=F_{1}^{\gamma} / 2$.

For the real photon case we have only one constraint, i.e.,, the first inequality of Eq. (4.59), which is rewritten as

$$
2\left|W_{3}^{\gamma}\right| \leq\left(W_{1}^{\gamma}+W_{4}^{\gamma}\right) .
$$

It is interesting to recall that the polarised structure function $W_{4}^{\gamma}$ of the real photon satisfies a remarkable sum rule [181]

$$
\int_{0}^{1} W_{4}^{\gamma}\left(x, Q^{2}\right) \mathrm{d} x=0 .
$$

The integral of $\left|W_{3}^{\gamma}\right|$ is, therefore, bounded from above by the first moment of $W_{1}^{\gamma}$,

$$
\int_{0}^{1}\left|W_{3}^{\gamma}\left(x, Q^{2}\right)\right| \mathrm{d} x \leq \frac{1}{2} \int_{0}^{1} W_{1}^{\gamma}\left(x, Q^{2}\right) \mathrm{d} x .
$$

These constraints could be studied in future experiments on the photon structure, through the twophoton processes in $\mathrm{e}^{+} \mathrm{e}^{-}$collisions as well as the resolved photon processes in a $\mathrm{e}^{-} \mathrm{p}$ collider. The validity of the inequality Eq. (4.63) has been checked in a simple parton model, as discussed in Ref. [177].

In the case of virtual photon, $p^{2}=-P^{2} \neq 0$, there appear eight structure functions (four of them are new) and we have derived three positivity constraints on these functions Eqs. (4.61). However, up to now very little attention has been paid to the virtual photon case and therefore, we have almost no knowledge of the new photon structure functions. In this situation it is worthwhile to investigate these new structure functions in the simple parton model to find out whether or not the three positivity constraints actually hold. They were proven to be satisfied in Ref. [178], both in a massive and massless quark cases, although the two cases show quite different behaviours.

\subsection{Positivity constraints for parton distributions and fragmentation func- tions}

In hard QCD processes, the transition from hadrons to quarks (antiquarks) and gluons is specified in terms of distribution functions and the transition from quarks (antiquarks) and gluons to hadrons is specified in terms of fragmentation functions. For example, the cross section for inclusive electroproduction $\mathrm{e} H \rightarrow \mathrm{e}^{\prime} X$, is given as a charge squared weighted sum over quark (and antiquark) 
distribution functions $q(x)$, describing the probability of finding quarks inside hadron $H$. Similarly, in electron-positron annihilation, the one-particle inclusive cross section $\mathrm{e}^{+} \mathrm{e}^{-} \rightarrow h X$ is given as a charge squared weighted sum over quark (and antiquark) fragmentation functions $D_{q}(z)$, describing the decay of the produced quarks (and antiquarks) into hadron $h$.

\subsubsection{Parton distributions and fragmentation functions (integrated over transverse momen- tum)}

The parton distribution functions (PDF) constitute the large-distance non-perturbative QCD inputs entering the expressions for the DIS structure functions, as well as for other hard QCD processes, in particular Semi-Inclusive DIS (SIDIS), Drell-Yan (DY) process and high $p_{T}$ hadron production in hadronic collisions.

For the derivation of positivity constraints for the PDF, we will combine the general operator method [182] with the approach based on helicity amplitudes, closely related to the consideration of structure functions, as used in Sec. 4.2.2.

i) - Unpolarised and polarised PDF Let us start the discussion with the simplest case of unpolarised quark distribution, which is, by introducing the light-cone decomposition of the quark fields [182], analogous to the scalar case. The quark forward distribution is just

$$
q(x)=\int \frac{\mathrm{d} \lambda}{4 \pi} \exp (i \lambda x)\langle p|\bar{\psi}(0) h \psi(\lambda n)| p\rangle=\frac{1}{\sqrt{2} p^{+}} \int \frac{\mathrm{d} \lambda}{2 \pi} \exp (i \lambda x)\left\langle p\left|\phi^{\dagger}(0) \phi(\lambda n)\right| p\right\rangle,
$$

where $\phi$ is the good component of the quark field and the light-cone vectors, $p$ and $n$ are normalised such as $p^{2}=0, n^{2}=0, p^{-}=n^{+}=0, p n=p^{+} n^{-}=1$. By inserting a complete set of intermediate states $|X\rangle$ of momentum $p_{X}$ and making use of the generalised optical theorem and the fact that the matrix elements may be replaced by their imaginary parts [183], the forward distribution can be written as

$$
q(x)=\sum_{X} \frac{1}{\sqrt{2} p^{+}}\left|\left\langle p\left|\phi^{\dagger}(0)\right| X\right\rangle\right|^{2} \delta\left(x-\left(p-p_{X}\right) \cdot n\right) .
$$

A comment is in order here. The states $|X\rangle$ are in fact coloured ones and therefore unphysical. One should therefore have in mind that the full unobserved state contains also the jet in the corresponding hard subprocess. The QCD factorisation implies the decomposition $|X\rangle \rightarrow\left|X_{h}\right\rangle\left|X_{s}\right\rangle$ to the states in the hard and soft jets. The soft colour neutralisation implies, in turn, the possibility to consider them separately. Therefore, in what follows these states should be understood as formal objects rather than physical objects, which is convenient for studies of positivity properties. Indeed, the expression (4.67) guarantees immediately the positivity of the spin-averaged quark distribution

$$
q(x) \geq 0 .
$$

In order to match the general framework of density matrix positivity (Sec. 2.2), one may interpret the quantity $q(x)$ as the diagonal element $q(x, x)$ of a generalised parton distribution matrix $q\left(x_{1}, x_{2}\right)$, which has infinite dimension, the continuous partonic variable $x \in[0,1]$ replacing the discrete index $n \in[1, N]$.

Note that our analysis can be simply extended to the case of fragmentation functions $D_{q}(z)$, describing the process $q \rightarrow h X$, which correspond to the following modification of Eq. (4.67)

$$
D_{q}(z)=\sum_{X} \frac{1}{\sqrt{2} p^{+}}|\langle 0|\phi(0)| p, X\rangle|^{2} \delta\left(1 / z-\left(p-p_{X}\right) . n\right) .
$$

Here $z=p / k$, where $k$ and $p$ are the quark and hadron $h$ momenta, respectively. As a result the spin averaged fragmentation function is also positive

$$
D_{q}(z) \geq 0,
$$


and all other positivity constraints which we will be considered for distribution functions, will have their counterparts for fragmentation functions (see, e.g., Ref. [149]).

The generalisation to the case of a longitudinally polarised proton of spin $S_{L}$ may be easily achieved, by considering the PDF with definite helicities $( \pm)$

$$
\begin{aligned}
q_{ \pm}(x) & =\int \frac{\mathrm{d} \lambda}{4 \pi} \exp (i \lambda x)\left\langle p, S_{L}\left|\bar{\psi}_{ \pm}(0) \not h \psi_{ \pm}(\lambda n)\right| p, S_{L}\right\rangle \\
& =\frac{1}{\sqrt{2} p^{+}} \int \frac{\mathrm{d} \lambda}{2 \pi} \exp (i \lambda x)\left\langle p, S_{L}\left|\phi_{ \pm}^{\dagger}(0) \phi_{ \pm}(\lambda n)\right| p, S_{L}\right\rangle
\end{aligned}
$$

where $\psi_{ \pm}=\left(1+\gamma^{5}\right) \psi / 2$ and $\phi_{ \pm}$are the respective good components, so that $q(x)=q_{+}(x)+q_{-}(x)$. It is now straightforward to get, in complete analogy to (4.67), the positivity of spin-dependent distributions which reads

$$
q_{ \pm}(x)=\sum_{X} \frac{1}{\sqrt{2} p^{+}}\left|\left\langle p, S_{L}\left|\phi_{ \pm}^{\dagger}(0)\right| X\right\rangle\right|^{2} \delta\left(x-\left(p-p_{X}\right) . n\right) \geq 0 .
$$

It is clear that now the helicity distributions correspond to diagonal elements of the density matrix, in the $x \otimes$ helicity basis. By introducing the more commonly used spin-dependent distribution $\Delta q(x)=$ $q_{+}(x)-q_{-}(x)$, one has

$$
q(x) \geq|\Delta q(x)|
$$

ii) - Quark transversity distribution and Soffer inequality Let us introduce the new ingredient in our analysis, corresponding to the possibility of non-diagonal elements of density matrices. The best known example is represented by the case of quark helicity flipping chiral-odd transversity distribution, defined as

$$
\begin{aligned}
\delta q(x) & =\int \frac{\mathrm{d} \lambda}{4 \pi} \exp (i \lambda x)\left\langle p, S_{T}\left|\bar{\psi}(0) \not h S \gamma_{5} \psi(\lambda n)\right| p, S_{T}\right\rangle \\
& =\Re \mathrm{e} \sum_{X} \frac{1}{\sqrt{2 p^{+}}}\left\langle p,+\left|\phi_{+}^{\dagger}(0)\right| X\right\rangle\left\langle p,-\left|\phi_{-}^{\dagger}(0)\right| X\right\rangle^{*} \delta\left(x-\left(p-p_{X}\right) . n\right) .
\end{aligned}
$$

where $S_{T}$ stands for the spin of the transversely polarised proton. The Soffer inequality [154]

$$
q_{+}(x)=(q(x)+\Delta q(x)) / 2 \geq|\delta q(x)|,
$$

is especially transparent, if the parton distributions are considered as the following bilinears of quark-hadron helicity amplitudes, corresponding $N \rightarrow q+X$,

$$
\begin{aligned}
q(x) & =\sum_{X}|\langle+1 / 2 \mid+1 / 2, X\rangle|^{2}+|\langle+1 / 2 \mid-1 / 2, X\rangle|^{2}, \\
\Delta q(x) & =\sum_{X}|\langle+1 / 2 \mid+1 / 2, X\rangle|^{2}-\sum_{X}|\langle+1 / 2 \mid-1 / 2, X\rangle|^{2}, \\
\delta q(x) & =2 \Re \mathrm{e} \sum_{X}\langle+1 / 2 \mid+1 / 2, X\rangle\langle-1 / 2 \mid-1 / 2, X\rangle^{*},
\end{aligned}
$$

where the unobserved state $|X\rangle$ is the same as discussed above. Now Soffer's inequality follows from the positivity of

$$
\sum_{X}|\langle+1 / 2 \mid+1 / 2, X\rangle \pm a\langle-1 / 2 \mid-1 / 2, X\rangle|^{2} \geq 0 .
$$

Note that only the amplitudes $|h, q\rangle$, with the same quark and hadron helicities enter (to) this equation due to helicity conservation resulting from angular momentum conservation in collinear kinematics. As the total helicity of both amplitudes is equal to the projection of angular momentum 


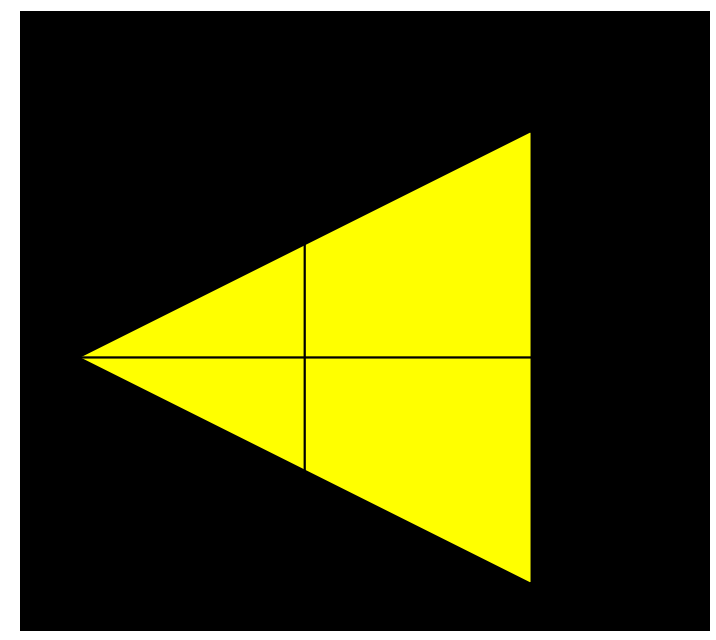

Figure 36: The allowed domain corresponding to the constraint Eq. (4.75).

of the state $|X\rangle$, the quark helicity flip in the interfering amplitudes should be accompanied by the hadron helicity flip. This explains why only the distribution $q_{+}=\sum_{X}|\langle+1 / 2 \mid+1 / 2, X\rangle|^{2}=$ $\sum_{X}|\langle-1 / 2 \mid-1 / 2, X\rangle|^{2}$ enters the Soffer inequality.

For completeness we show in Fig. 36 that the region restricted by Eq. (4.75) is only half of the entire square.

iii) - Bounds for different polarised gluon distributions One can easily generalise these considerations to the case of offshell gluons, when respective distributions are also related to the helicity amplitudes, corresponding to $N \rightarrow G+X$,

$$
\begin{aligned}
G_{ \pm}(x) & =\sum_{X}|\langle+1 / 2 \mid \pm 1, X\rangle|^{2}, \\
G_{L}(x) & =\sum_{X}|\langle+1 / 2 \mid 0, X\rangle|^{2}=\sum_{X}|\langle-1 / 2 \mid 0, X\rangle|^{2}, \\
\Delta G_{T} & =\Re \mathrm{e} \sum_{X}\langle+1 / 2 \mid+1, X\rangle\langle-1 / 2 \mid 0, X\rangle^{*},
\end{aligned}
$$

where $G_{ \pm}(x)=(G(x) \pm \Delta G(x)) / 2$ are combinations of the well known spin-averaged $G$ and longitudinally polarised $\Delta G$ gluon distributions, $\Delta G_{T}$ is the counterpart of the latter for transverse polarised nucleon [184], while $G_{L}$ is the distribution of longitudinal (scalar) gluons in a nucleon [185]. $\Delta G_{T}$ is related to the distributions $H_{1}$ and $H_{2}$ introduced in [186]. We can now write the CS inequality as

$$
\sum_{X}|\langle+1 / 2 \mid+1, X\rangle \pm a\langle-1 / 2 \mid 0, X\rangle|^{2} \geq 0 .
$$

By performing the minimisation with respect to the variation of $a$, which was unnecessary in the case of Soffer inequality, because the minimum was provided for $a=1$, we obtain the following bound

$$
\left|\Delta G_{T}(x)\right| \leq \sqrt{G_{+}(x) G_{L}(x)} .
$$

This was first derived [187] in a weaker form with $G$ instead of $G_{+}$, whose appearance is again due to helicity conservation, since only the state with parallel helicities of nucleon and gluon may interfere with some other helicity state which is $|-1 / 2,0\rangle$. This bound is completely similar to the bound for $A_{2}$ mentioned in Sec. 4.2.2. 
Note that because the scalar gluon polarisation is not enhanced by Lorentz boost contrary to the transverse one, it produces the distributions of higher twist-three $\left(\Delta G_{T}\right)$ and twist-four $\left(G_{L}\right)$ which are related by positivity constraints, like in the case of $A_{2}$. It is most instructive to use it to bound the less known twist-four distribution from above [187]

$$
G_{L}(x) \geq\left[\Delta G_{T}(x)\right]^{2} / G_{+}(x) .
$$

Another application of similar reasoning may be used in the case of twist-three single spin asymmetries (SSA), which are enhanced at large $x$, to deduce that twist-four corrections to unpolarised cross-sections should be also large in this region [188]. This may explain the observation [189] that the NLO QCD calculation largely underestimates the pion production in unpolarised hadronic collisions at lower energies, where large SSA are observed, whereas it describes it well at higher energies, where SSA are smaller. Indeed, the lower energies at the same $p_{T}$ mean the higher typical partonic $x$. In that region enhanced twist-three leads to SSA while simultaneously enhanced, because of positivity, twist-four, spoils the NLO QCD applicability. It is known that the account for intrinsic partonic transverse momentum improves the description of spin-averaged data [190]. At the same time, such intrinsic momentum may be considered [191] as a model for infinite resummed tower of higher twists, supporting the suggested picture.

In order to avoid the contribution of scalar gluons polarisation and to have gluonic helicity flip effects at leading twist level one should be able to compensate the helicity flip two of the gluon, which is possible in the case of a spin one target like the deuteron [192]. The polarised leading twist gluonic distributions in spin one hadron take the form

$$
\begin{aligned}
G_{ \pm}^{1}(x) & =\sum_{X}|\langle+1 \mid \pm 1, X\rangle|^{2}, \\
\delta G_{T}^{1}(x) & =\Re \mathrm{e} \sum_{X}\langle+1 \mid+1, X\rangle\langle-1 \mid-1, X\rangle^{*},
\end{aligned}
$$

where $\delta G_{T}^{1}(x)=G_{x / x}(x)-G_{y / x}(x)$ and $G_{n / x}(x)$ is the density of gluon linearly polarised along $\boldsymbol{n}$, in a spin-one hadron linearly polarised along $\hat{\boldsymbol{x}}$. It can be called the leading twist gluonic transversity; it has an evolution kernel close to $\delta q(x)[70]$ and an analogue of the Soffer inequality

$$
G_{+}^{1}(x)=\left(G^{1}(x)+\Delta G^{1}(x)\right) / 2 \geq\left|\delta G_{T}^{1}(x)\right|,
$$

may be derived in complete similarity with its quark counterpart.

\subsubsection{Spin and transverse-momentum dependent quark distributions}

The spin- and $\boldsymbol{k}_{T}$-dependent quark distributions in the nucleon can be viewed as the probability of the virtual inclusive process

$$
\operatorname{nucleon}(\boldsymbol{p}, \boldsymbol{S}) \rightarrow \operatorname{quark}\left(\boldsymbol{k}, \boldsymbol{S}^{\prime}\right)+X,
$$

with $\boldsymbol{k}=x \boldsymbol{p}+\boldsymbol{k}_{T}$. This reaction has the same spin content as the inclusive reaction (4.8). The $\left(\boldsymbol{p}, \boldsymbol{k}_{T}\right)$ plane is a symmetry plane and plays the same role as the scattering plane in (4.8). Therefore the spin observables of (4.84) obey the same parity constraints and inequalities as (4.8). The distribution of quarks of polarisation $\boldsymbol{S}^{\prime}$ in a nucleon of polarisation $S$ can be written in terms of the unpolarised quantity $q\left(x,\left|\boldsymbol{k}_{T}\right|\right)$ and seven Cartesian parameters $D_{i j}$

$$
\begin{aligned}
\frac{\mathrm{d} N}{\mathrm{~d} x \mathrm{~d}^{2} \boldsymbol{k}_{T}} \equiv & q\left(x, \boldsymbol{k}_{T}, \boldsymbol{S}, \boldsymbol{S}^{\prime}\right)=\frac{q\left(x,\left|\boldsymbol{k}_{T}\right|\right)}{2}\left[1+D_{0 n} \boldsymbol{S}^{\prime} . \boldsymbol{n}+D_{n 0} \boldsymbol{S} . \boldsymbol{n}+D_{n n} \boldsymbol{S} . \boldsymbol{n} \boldsymbol{S}^{\prime} . \boldsymbol{n}\right. \\
& \left.+D_{l l} \boldsymbol{S} . \boldsymbol{l} \boldsymbol{S}^{\prime} . \boldsymbol{l}+D_{l m} \boldsymbol{S} . \boldsymbol{l} \boldsymbol{S}^{\prime} \cdot \boldsymbol{m}+D_{m l} \boldsymbol{S} . \boldsymbol{m} \boldsymbol{S}^{\prime} . \boldsymbol{l}+D_{m m} \boldsymbol{S} . \boldsymbol{m} \boldsymbol{S}^{\prime} \boldsymbol{m}\right]
\end{aligned}
$$

The $D_{i j}$ 's also are functions of $x$ and $\left|\boldsymbol{k}_{T}\right|$. The dependence of the quark density with respect to the azimuth of $\boldsymbol{k}_{T}$ at given $\boldsymbol{S}$ and $\boldsymbol{S}^{\prime}$ is contained in the factors $\boldsymbol{S} . \boldsymbol{m}, \boldsymbol{S} . \boldsymbol{n}, \boldsymbol{S}^{\prime} . \boldsymbol{m}$ and $\boldsymbol{S}^{\prime} . \boldsymbol{n}$, where 
$\boldsymbol{l}=\boldsymbol{p}_{T} /|\boldsymbol{p}|, \boldsymbol{m}=\boldsymbol{k}_{T} /\left|\boldsymbol{k}_{T}\right|$ and $\boldsymbol{n}=\boldsymbol{l} \times \boldsymbol{m}$. The Cartesian parameters are related to the functions $f_{1}, f_{1 T}^{\perp}$, etc., of the Amsterdam notation (see, e.g., $[48,193]$ ) by

$$
\begin{aligned}
f_{1}\left(x, k_{T}\right) & =q\left(x, k_{T}\right), \\
g_{1}\left(x, k_{T}\right) & =q\left(x, k_{T}\right) D_{l l}, \\
h_{1}\left(x, k_{T}\right) & =q\left(x, k_{T}\right)\left(D_{m m}+D_{n n}\right) / 2, \\
\left(k_{T}^{2} /\left(2 M^{2}\right)\right) h_{1 T}^{\perp}\left(x, k_{T}\right) & =q\left(x, k_{T}\right)\left(D_{m m}-D_{n n}\right) / 2, \\
\left(k_{T} / M\right) g_{1 T}\left(x, k_{T}\right) & =q\left(x, k_{T}\right) D_{m l}, \\
\left(k_{T} / M\right) h_{1 L}^{\perp}\left(x, k_{T}\right) & =q\left(x, k_{T}\right) D_{l m}, \\
\left(k_{T} / M\right) h_{1}^{\perp}\left(x, k_{T}\right) & =-q\left(x, k_{T}\right) D_{0 n}, \\
\left(k_{T} / M\right) f_{1 T}^{\perp}\left(x, k_{T}\right) & =q\left(x, k_{T}\right) D_{n 0},
\end{aligned}
$$

where $k_{T}=\left|\boldsymbol{k}_{T}\right|$. The integrals of the first three functions over $\boldsymbol{k}_{T}$ give $q(x), \Delta q(x)$ and $\delta q(x)$. The last function describes the Sivers effect $[68,157]$. The $4^{\text {th }}$ to $7^{\text {th }}$ functions were introduced by Kotzinian [194], Mulders, Boer and Tangerman $[69,195]$. The $4^{\text {th }}$ one, $h_{1 T}^{\perp}$, has been given the name pretzelosity [196]. The positivity constraints have been first derived in the Amsterdam notation by Bacchetta et al. [193]. Using the Cartesian parameters they take the simple form [31]

$$
\left(1 \pm D_{n n}\right)^{2} \geq\left(D_{0 n} \pm D_{n 0}\right)^{2}+\left(D_{l l} \pm D_{m m}\right)^{2}+\left(D_{l m} \mp D_{m l}\right)^{2}
$$

together with the trivial bounds $\left|D_{i j}\right| \leq 1$. These inequalities are easily derived in the transversity basis and have the same form as (4.11).

Similar formulas also apply to the quark fragmentation $q \rightarrow$ baryon $+X$, just by interchanging the quark and the nucleon.

An example: the relativistic (quark + scalar diquark) model See [70,197-201]. The simplest spectator system $X$ is a $0^{+}$diquark, like the $(u d)$ pair of the $\Lambda$ baryon in the SU(6) model. Assuming a quark-baryon-diquark vertex of the form $g\left(k^{2}\right) \bar{u}\left(k, \boldsymbol{S}^{\prime}\right) u(p, \boldsymbol{S})$, the quark polarisation $\boldsymbol{S}^{\prime}$ is obtained from $\boldsymbol{S}$ by a rotation of angle $\theta=-2 \arctan \left[k_{T} /\left(m_{q}+x m_{B}\right)\right]$ in the $\left(\boldsymbol{p}, \boldsymbol{k}_{T}\right)$ plane, as seen from formula (43-44) of [72]. This model gives $D_{n n}=1, D_{l l}=D_{m m}=\cos \theta$, $D_{l m}=-D_{m l}=\sin \theta$ and predicts no Sivers or Boer-Mulders effect: $D_{n 0}=D_{0 n}=0$, thus satisfying Hermiticity and time reversal invariance (see Sec. 2). Note that this model exhibits a strong "spin crisis" (small $D_{l l}$ ) at large $\left\langle k_{T}\right\rangle / M$. The positivity constraints (4.87) as well as the Soffer bound are saturated. A pure baryon spin state $(|S|=1)$ leads to a pure quark spin state $\left(\left|S^{\prime}\right|=1\right)$. The reason is that a spin-zero spectator cannot take away any spin information. This is no more the case when the model is enriched with $1^{+}$spectator diquarks, as in [197-200], or in a more realistic model with gluons and sea quarks. The corresponding kinematical domain corresponds to small $x$, and one expects Soffer's inequality far from saturation there.

Adding the gluon field also gives rise to non-intrinsic Sivers $\left(D_{n 0} \neq 0\right)$ and Boer-Mulders $\left(D_{0 n} \neq 0\right)$ effects. In deep inelastic electron scattering, the Sivers effect is due to the final state interaction of the scattered quark with spectator partons $[159,202]$. In the Drell-Yan lepton pair production, the Sivers effect in the target is due to the initial state interaction between the active quark of the projectile with the spectators of the target, and its sign is opposite to the DIS one [203]. For references, see also [48]. A "theoretical laboratory" [204,205] to study these relativistic effects is the large- $Z$ hydrogen-like atom, where the electron plays the role of a parton. This model has many common features with the models describing the nucleon with a quark and a scalar diquark. It also possesses a photon cloud and an electron-positron sea, interpreted as the local deformation of the Dirac sea.

Role of the Sivers and Collins effects in single-spin asymmetries The various functions of (4.86) can be separated unambiguously by their different azimuthal dependences in the semi-inclusive deep 
inelastic scattering experiments being presently performed at HERMES [206], Jlab [18], COMPASS [207-210]. Their roles in the single-spin asymmetries like $\mathrm{p} \uparrow+\mathrm{p} \rightarrow \pi+X$ at $p_{T} \sim 1-2 \mathrm{GeV}$ is still controversial. A model based on the Collins effect and a large transversity distribution $\delta q(x)[163$, 164] could reproduce the E704 experiment [211,212], but was hardly compatible with the Soffer bound [213]. An alternative model based on the Sivers effect gave reasonable results [160,190]. See, also, [214]. On the other hand, the model of [164] can be improved by taking into account the azimuthal $k_{T}$-dependence of the polarised quark distribution [215]. Let the proton spin points in the $\hat{\boldsymbol{y}}$ direction and the pion be produced in the $(z, x)$ plane. Allowing for a semi-hard collision of momentum transfer $q=k^{\prime}-k$ and assuming that the quark fragments into a pion of transverse momentum $\tilde{\boldsymbol{p}}_{T}$ relative to $k^{\prime}$, one has $\boldsymbol{p}_{T} \simeq(p / k)\left(\boldsymbol{k}_{T}+\boldsymbol{q}_{T}\right)+\tilde{\boldsymbol{p}}_{T}$. Due to the trigger bias, $\boldsymbol{k}_{T}$ points preferably towards $\boldsymbol{p}_{T}$, therefore is mainly in the $(z, x)$ plane. Accordingly the quark polarisation is $S_{q} \simeq D_{n n}\left(x, \boldsymbol{k}_{T}\right) \hat{\boldsymbol{y}}$. Scattering does not change $S_{q}$ too much and one gets a Collins effect of strength $\left\langle\tilde{\boldsymbol{p}}_{T}\right\rangle \propto D_{n n}\left(x, \boldsymbol{k}_{T}\right)$ instead of $\delta q(x) / q(x)$. Contrary to $\delta q(x) / q(x), D_{n n}\left(x, \boldsymbol{k}_{T}\right)$ is not constrained by the Soffer bound, as illustrated by the quark-scalar diquark model where $D_{n n}=1$. Therefore the Collins effect combined with $k_{T}$-dependent polarised quark distribution bypasses the Soffer bound and could well be responsible for a dominant part of the single-spin asymmetry.

T-odd distributions functions Here it is worth mentioning the case of T-odd fragmentation functions ${ }^{24}$, which was given recently special attention. It is a new topic which belongs to the field one could call quark and gluon polarimetry. This is a consequence of possible final state interactions in the fragmentation process, giving rise to a non-trivial phase. A particular example of such a function is the Collins function [158], which describes the following process $q(\uparrow) \rightarrow h X$, namely the fragmentation of a transversely polarised quark into an unpolarised hadron $h$ (e.g., a pion). In this case, provided $h$ has a transverse momentum $\mathbf{p}_{\mathbf{T}}$, if $\boldsymbol{S}_{T}^{q}$ denotes the transverse spin of the quark, the event rate reads

$$
\mathrm{d} N(q(\uparrow) \rightarrow h X)=\mathrm{d} z \mathrm{~d} \boldsymbol{p}_{T} D\left(z, \boldsymbol{p}_{T}\right)\left[1+A_{C}\left(z, p_{T}\right) \frac{\left(\boldsymbol{k} \times \boldsymbol{p}_{T}\right) \cdot \boldsymbol{S}_{T}^{q}}{\left|\boldsymbol{k} \times \boldsymbol{p}_{T}\right|}\right],
$$

where $\left|A_{C}\right| \leq 1$ and $D A_{C}$ is the Collins function. More generally, one introduces the concept of jet handedness [216], an old idea first proposed by Nachtmann [217], which can be used to determine the polarisation of a high energy quark or gluon. It is due to the correlations between the spin of the fragmenting parton and the momenta of the produced hadrons and for example the quark helicity can be obtained from the sign of $\boldsymbol{p}^{(1)} \cdot\left(\boldsymbol{p}^{(2)} \times \boldsymbol{p}^{(3)}\right)$, where $\boldsymbol{p}^{(1)}, \boldsymbol{p}^{(2)}$ and $\boldsymbol{p}^{(3)}$ are the three leading meson momenta. Positivity requires that these functions cannot exceed the corresponding spin-averaged functions.

\subsubsection{Generalised Parton Distributions}

Let us finally consider the possibility of non-diagonality in the momentum, rather than in helicity, which is the case of generalised parton distributions (GPD) representing the most general nonperturbative QCD inputs allowing to describe the amplitudes of exclusive hard processes. The review of the positivity bounds for these objects may be found in Sec. 3.11 of [218]. Here we simply describe the derivation of positivity bounds for GPD, with the use of the operator method [182] and we mention some new versions of these inequalities.

The unpolarised quark GPD reads

$$
H_{q}(x, \xi)=\frac{1}{\sqrt{1-\xi^{2}}} \int \frac{\mathrm{d} \lambda}{4 \pi} \exp (i \lambda x)\left\langle p^{\prime}|\bar{\psi}(0) h \psi(\lambda n)| p\right\rangle,
$$

where the factor $\sqrt{1-\xi^{2}}$ comes from the bilinear $\bar{u}\left(p^{\prime}\right) u(p)$, in the definition of the GPD [218,219]. We have assumed the strictly collinear kinematics, so that $p=P(1+\xi)$ and $p^{\prime}=P(1-\xi)$ with $P=\left(p+p^{\prime}\right) / 2$.

\footnotetext{
${ }^{24} \mathrm{~T}$-odd distribution functions, arising from initial state interactions, will be discussed in Sec. 4.3.2
} 
By an analogous procedure as above, it becomes

$$
H_{q}(x, \xi)=\Re \mathrm{e} \sum_{X} \frac{1}{\sqrt{2\left(1-\xi^{2}\right)} p^{+}}\langle p|\phi(0)| X\rangle\left\langle p^{\prime}|\phi(0)| X\right\rangle^{*} \delta\left(x-\left(p-p_{X}\right) . n\right),
$$

where we used the hermiticity of the matrix element.

We are now ready to write down [220] the Cauchy-Schwarz inequality as follows

$$
\sum_{X}\left|\langle p|\phi(0)| X\rangle \pm a\left\langle p^{\prime}|\phi(0)| X\right\rangle\right|^{2} \delta\left(x-\left(p-p_{X}\right) . n\right) \geq 0
$$

where $a$ is a positive number. While the non-diagonal term of (4.91) is just producing the GPD, the diagonal terms are related to the usual (diagonal) parton distributions. Performing the minimisation with respect to the variation of $a$, we finally get

$$
\left|H_{q}(x, \xi)\right| \leq \sqrt{\frac{q\left(x_{1}\right) q\left(x_{2}\right)}{1-\xi^{2}}},
$$

where $x_{1,2}=(x \pm \xi) /(1 \pm \xi)$. The positivity constraints should be generalised if one goes beyond the collinear approximation for the initial and final momentum, when the helicity-flip GPD, denoted by E, starts to contribute [221-223]. The general analysis of the parton-hadron eight-dimensional helicity matrix, which by use of symmetry properties, may be reduced to dimensions four and two, allows to get [224] the complete set of inequalities involving the full basis of GPD's. Another general framework for the derivation of positivity constraints is also provided by impact space representation [225].

The derivation for the gluons is analogous [220, 224, 226-229]. The corresponding positivity conditions will be discussed in more detail in Sec. 4.3.4, when QCD evolution will be considered.

Finally, let us consider the possibility of transition GPD [219], when not only momentum but also the type of the hadron can be different

$$
H_{A B}(x, \xi)=\frac{1}{\sqrt{1-\xi^{2}}} \int \frac{\mathrm{d} \lambda}{4 \pi} \exp (i \lambda x)\left\langle B, p^{\prime}|\bar{\psi}(0) \pitchfork \psi(\lambda n)| A, p\right\rangle .
$$

It is straightforward to bound them by the distributions for hadrons $A$ and $B$

$$
\left|H_{A B}(x, \xi)\right| \leq \sqrt{\frac{q_{A}\left(x_{1}\right) q_{B}\left(x_{2}\right)}{1-\xi^{2}}} .
$$

This bound was recently studied [230] for the case of pion-photon transition GPD [231] and the consideration of photonic GPD in the perturbative regime allows explicit tests of these inequalities [232].

\subsubsection{QCD Evolution of Positivity Constraints}

One may wonder, to what extent the $Q^{2}$ evolution of QCD is compatible with the positivity constraints. In this section it is shown, that positivity becomes especially clear when the evolution equation is interpreted as a kinetic master equation. In particular, one obtains a complete proof of the corresponding inequalities, at leading order (LO) and also at next-to-leading order (NLO), provided a suitable factorisation scheme is chosen. New positivity properties, which currently may be proved only at the perturbative QCD level, will be discussed. They include preservation of positivity by the Balitsky-Fadin-Kuraev-Lipatov (BFKL) equation [233-235] (see v) below) and the convexity of parton distributions (see vi) below). The important property of positivity in QCD is that it is preserved only in one direction of evolution, providing a sort of "scale arrow". 
i) - Master equations for QCD evolution The crucial point in preserving the positivity property is that all the known evolution equations may be represented [188,236-238] in a form of master, or gain-loss equation, like

$$
\frac{\mathrm{d} q(x, t)}{\mathrm{d} t}=\int \mathrm{d} y[w(y \rightarrow x) q(y, t)-w(x \rightarrow y) q(x, t)] .
$$

Here $q$ is a generic parton distribution and the role of "time" $t$ is played either by the longitudinal momentum fraction for the BFKL equation [239] or by the transverse momentum for the DokshitzerGribov-Lipatov-Altarelli-Parisi (DGLAP) equations [240-242]. The probabilistic understanding of the DGLAP evolution equations came already from some pioneering papers [240-244]. Actually, its standard form ${ }^{25}$ (for the time being, we shall confine ourselves to the non-singlet (NS) case)

$$
\frac{\mathrm{d} q(x)}{\mathrm{d} t}=\frac{\alpha_{s}}{2 \pi} \int_{x}^{1} \mathrm{~d} y \frac{q(y)}{y} P(x / y)
$$

may be interpreted as a "time" $t=\ln Q^{2}$ evolution of the "particle" density $q$ in the one-dimensional space $0 \leq x \leq 1$, due to the flow from right to left, with the probability equal to the splitting kernel $P$. The key element of such interpretation is the problem of the infrared (IR) singular terms in $P$, which was considered in detail some years ago [245] (see also [246]). The kinetic interpretation is obviously preserved provided the "+" form of the kernel is presented in the following way

$$
P_{+}(z)=P(z)-\delta(1-z) \int_{0}^{1} P(y) \mathrm{d} y
$$

leading to the corresponding expression for the evolution equation

$$
\frac{\mathrm{d} q(x)}{\mathrm{d} t}=\frac{\alpha_{s}}{2 \pi}\left[\int_{x}^{1} \mathrm{~d} y \frac{q(y)}{y} P(x / y)-q(x) \int_{0}^{1} P(z) d z\right] .
$$

This form has already a kinetic interpretation because the second term in the square brackets describes the flow of the partons at the point $x$ [245]. It seems however instructive to make this similarity even clearer by the simple change of variables $z=y / x$ in the second term, which leads to the following symmetric form

$$
\frac{\mathrm{d} q(x)}{\mathrm{d} t}=\frac{\alpha_{s}}{2 \pi}\left[\int_{x}^{1} \mathrm{~d} y \frac{q(y)}{y} P(x / y)-\int_{0}^{x} \mathrm{~d} y \frac{q(x)}{x} P(y / x)\right] .
$$

It allows us to write it down in a form of master equation (see Eq. (4.95)) with the 'transition probability' defined as

$$
w(y \rightarrow x)=\frac{\alpha_{s}}{2 \pi} P(x / y) \frac{\theta(y-x)}{y} .
$$

As a result, the known properties of the evolution equation receive the following simple interpretation: the cancellation of the IR divergences between the contributions of the real and virtual gluons emission is coming from the equality of in- and out- flows, when in both terms of Eq. (4.95) one has $y \sim x$, following from the continuity condition for the "particle" number. Also, the conservation of the vector current

$$
\int_{0}^{1} \mathrm{~d} x \frac{\mathrm{d} q(x)}{\mathrm{d} t}=\int_{0}^{1} \int_{0}^{1} \mathrm{~d} x \mathrm{~d} y[w(y \rightarrow x) q(y)-w(x \rightarrow y) q(x)]=0,
$$

comes from the integration of an antisymmetric function in a symmetric region.

\footnotetext{
${ }^{25}$ For brevity, the argument $t$ will not be written down explicitly in the parton densities.
} 
As the transition probability is peaked for $x \sim y$, which is the reflection of the IR divergence, it is natural to apply the Kramers-Moyal (KM) expansion [247]. However, the natural expansion variable is $Y=\ln x$ [238], rather than $x$ as in Ref. [247]. The reason is that the transition probability acquires than the translation invariant form. The master equation holds for function $f(Y)=x q(x)$, with $x=\exp (Y)$, as its integral over $Y$ conserves

$$
\int_{0}^{1} \mathrm{~d} x q(x)=\int_{-\infty}^{0} \mathrm{~d} Y f(Y)
$$

By rewriting Eq. (4.101) in terms of the new variable $Y$, one may expand the first term in power series in $Y$, which is just the KM expansion, and extend the integration to the whole $Y$ axis $(-\infty<Y<$ $\infty)$. If the initial distribution remains non-zero only for $0<x<1(-\infty<Y<0)$, the directional property of the DGLAP equation, resulting from the $\theta$-function in Eq. (4.100), guarantees that the evolved function also remains non-zero only for $0<x<1(-\infty<Y<0)$. As a result, the moments of transition probability appearing in the KM expansion, coincide with the derivatives of anomalous dimension at $n=1$

$$
\int_{0}^{1} \mathrm{~d} x \ln ^{k} x P(x)=-\left.\frac{2 \pi}{\alpha_{s}} \frac{\mathrm{d}^{k}}{\mathrm{~d} n^{k}} \gamma(n)\right|_{n=1}, \quad \text { with } \quad \gamma(n)=-\frac{\alpha_{s}}{2 \pi} \int_{0}^{1} P(x) x^{n-1} \mathrm{~d} x,
$$

which leads to the differential form of DGLAP equation

$$
\frac{\partial q(x)}{\partial t}=-\left.\frac{1}{x} \exp \left[\frac{\partial}{\partial \ln (1 / x)} \frac{\mathrm{d}}{\mathrm{d} n}\right] x q(x) \gamma(n)\right|_{n=1} .
$$

The Fokker-Planck (diffusion) approximation corresponds to keeping only the two first terms in this equation. Substituting the expression for LO non-singlet kernel to (4.103) with $n=1,2$ one gets

$$
\begin{gathered}
\frac{\partial q(x)}{\partial t}=\frac{1}{x}\left(v \frac{\partial(x q(x))}{\partial \ln (1 / x)}+D \frac{\partial^{2}(x q(x))}{\partial \ln ^{2}(1 / x)}\right), \\
\text { with } \quad v=\frac{\alpha_{s}}{2 \pi}\left(5 / 4-\pi^{2} / 3\right), \quad \text { and } \quad D=\frac{\alpha_{s}}{4 \pi}(-9 / 8+2 \zeta(3)) .
\end{gathered}
$$

Here $v$ stands for the drift velocity. Note that the positivity of the diffusion coefficient $D$ naturally explains the convexity of the curve of anomalous dimension, first pointed by Nachtmann [248,249]. It is also possible to observe the convexity of the anomalous dimension curve in the points different from $n=1$. In this case, one should consider the evolution of the function $x^{k} q(x)$ with arbitrary positive $k$ and perform a similar KM expansion. Note that besides the diffusion and drift effects one will get also the "decay" effect, since the corresponding moment is not conserved.

ii) - Preservation of positivity constraints for spin-dependent distributions by QCD evolution Let us start with obvious remarks about the unpolarised distributions. As soon as the master equation has a clear probabilistic meaning, and the probabilities of the corresponding Markovian process are always positive, the preservation of positivity becomes clear.

For further analysis it is, however, instructive, to describe in some detail the formal reasons of this preservation. The negative second term in Eq. (4.95) cannot change the sign of the distribution because it is 'diagonal' in $x$ (recall that $x$ may be considered as a label of density matrix), which means that it is proportional to the function at the same point $x$, as in the l.h.s.. When the distribution gets too close to zero, its stops decreasing. This is true for both ${ }^{\prime}{ }^{\prime}$ and $\delta(1-z)$ terms, for any value of their coefficient (if it is positive, it will reinforce the positivity of the distribution).

Let us consider now the spin-dependent case. For simplicity, we postpone the discussion of quark-gluon mixing for a moment, but allow the spin-dependent and spin-independent kernels to be different, as they are at NLO. It is most convenient to write down the equations for definite parton helicities, which was actually the starting point in deriving the equations for the spin-dependent 
quantities [241]. Although the form, which we shall use, mixes the contributions of different helicities, it makes the positivity properties especially clear. So we have

$$
\frac{\mathrm{d} q_{ \pm}(x)}{\mathrm{d} t}=\frac{\alpha_{s}}{2 \pi}\left[P_{+ \pm}(x / y) \otimes q_{+}(y)+P_{+\mp}(x / y) \otimes q_{-}(y)\right] .
$$

Here $P_{+ \pm}(z)=(P(z) \pm \Delta P(z)) / 2$, are the evolution kernels for definite helicities, and the shorthand notation for the convolution is adopted. As soon as $x<y$, the positivity of the initial distributions $\left(q_{ \pm}\left(x, Q_{0}^{2}\right) \geq 0\right.$, or $\left.\left|\Delta q\left(x, Q_{0}^{2}\right)\right| \leq q\left(x, Q_{0}^{2}\right)\right)$ is preserved, if both kernels $P_{+ \pm}$are positive, which is true, if

$$
|\Delta P(z)| \leq P(z), \quad z<1 .
$$

The singular terms at $z=1$ are not altering positivity, because they appear only in the diagonal (now in helicities) kernel $P_{++}$(only forward scattering is IR dangerous). From the kinetic interpretation again the distributions $q_{+}, q_{-}$stop decreasing, as soon as they are close to changing sign. From the general point of view, the quantities in the l.h.s. of Eq. (4.106) may be considered as diagonal elements of density matrix remaining positive in the course of the QCD evolution.

Now to extend the proof to the quark gluon mixing is trivial. One should write down the expressions for the evolutions of quark and gluon distributions of each helicity

$$
\begin{gathered}
\frac{\mathrm{d} q_{ \pm}(x)}{\mathrm{d} t}=\frac{\alpha_{s}}{2 \pi}\left[P_{+ \pm}^{q q}(x / y) \otimes q_{+}(y)+P_{+\mp}^{q q}(x / y) \otimes q_{-}(y)\right] \\
+P_{+ \pm}^{q G}(x / y) \otimes G_{+}(y)+P_{+\mp}^{q G}(x / y) \otimes G_{-}(y) \\
\frac{\mathrm{d} G_{ \pm}(x)}{\mathrm{d} t}=\frac{\alpha_{s}}{2 \pi}\left[P_{+ \pm}^{G q}(x / y) \otimes q_{+}(y)+P_{+\mp}^{G q}(x / y) \otimes q_{-}(y)\right. \\
\left.+P_{+ \pm}^{G G}(x / y) \otimes G_{+}(y)+P_{+\mp}^{G G}(x / y) \otimes G_{-}(y)\right] .
\end{gathered}
$$

If the inequality (4.107) is valid for each type of partons [237],

$$
\left|\Delta P^{i j}(z)\right| \leq P^{i j}(z), \quad z<1 ; \quad i, j=q, G,
$$

all the kernels, appearing in the r.h.s. of such a system, are positive. Concerning the singular terms, they are again diagonal, now in parton type, and do not affect positivity. The validity of these equations at LO comes just from the way they were derived, as the (positive) helicity-dependent kernels were in fact first calculated in Ref. [241]. At NLO, the situation is more controversial [237] and positivity can be used for the choice of factorisation scheme. The positivity is, generally speaking, not preserved order by order, but is typically preserved for the sum of LO and NLO terms.

To conclude, the stability of positivity under $Q^{2}$ evolution comes from two sources: i) the inequalities (4.109), leading to the increasing of distributions, ii) the kinetic interpretation of the decreasing terms. For the latter it is crucially important, that they are diagonal in $x$, in helicity and in parton type, which is related to their IR nature. Such property of virtual correction makes preservation of positivity especially simple when the evolution in $x$ space is considered, while in the space of the moments of parton distributions it requires a more elaborate analysis [248, 249].

iii) - Preservation of Soffer inequality by QCD evolution Let us now come to the evolution of Soffer inequality. This is really a crucial problem as the very different evolution of transversity lead to the suggestion that it cannot be stable against QCD evolution [250], However, this evolution, albeit different, is very precisely correlated with the evolution of chiral-even distributions leading to the stability of Soffer inequality.

According to the previous analysis it is straightforward [236] to define the following 'super'distributions, corresponding to the eigenvalues of density matrix:

$$
Q_{ \pm}(x)=q_{+}(x) \pm h_{1}(x) .
$$


Due to Soffer inequality, both these distributions are positive at some point $Q_{0}^{2}$, and the evolution equations for the NS case take the form

$$
\frac{\mathrm{d} Q_{ \pm}(x)}{\mathrm{d} t}=\frac{\alpha_{s}}{2 \pi}\left(P_{+ \pm}^{Q}(x / y) \otimes Q_{+}(y)+P_{+\mp}^{Q}(x / y) \otimes Q_{-}(y)\right),
$$

where the 'super'-kernels at LO are just

$$
\begin{aligned}
& P_{++}^{Q}(z) \equiv \frac{P_{q q}^{(0)}(z)+P_{h}^{(0)}(z)}{2}=\frac{C_{F}}{2}\left[\frac{(1+z)^{2}}{(1-z)_{+}}+3 \delta(1-z)\right], \\
& P_{+-}^{Q}(z) \equiv \frac{P_{q q}^{(0)}(z)-P_{h}^{(0)}(z)}{2}=\frac{C_{F}}{2}(1-z) .
\end{aligned}
$$

One can easily see [236], that the inequalities analogous to Eq. (4.109) are satisfied, so that both $P_{++}^{Q}(z)$ and $P_{+-}^{Q}(z)$ [251] are positive for $z<1$, while the singular term does appear only in the diagonal kernel. So, both requirements are valid and Soffer inequality is preserved under LO evolution. The extension to the singlet case is trivial, as the chiral-odd transversity distributions does not mix with gluons. Therefore, they affect only the evolution of quarks, and lead to the presence in the r.h.s. of the same extra terms as in Eq. (4.108). We have now

$$
\begin{aligned}
\frac{\mathrm{d} Q_{ \pm}(x)}{\mathrm{d} t}=\frac{\alpha_{s}}{2 \pi}\left[P_{+ \pm}^{Q}(x / y) \otimes Q_{+}(y)\right. & +P_{+\mp}^{Q}(x / y) \otimes Q_{-}(y) \\
& \left.+P_{+-}^{q G}(x / y) \otimes G_{+}(y)+P_{++}^{q G}(x / y) \otimes G_{-}(y)\right],
\end{aligned}
$$

where the non-diagonal kernels are all positive and free from singular terms, so positivity is preserved. This completes the proof of the positivity at leading order, while the NLO case is considered in detail in [236] (see also [252,253]).

iv)- Preservation of positivity constraints for Generalized Parton Distributions by QCD evolution We consider the evolution of the constraints for gluonic GPD, thus completing our discussion of quark GPD's in Sec. 4.3.3. We restrict ourselves to the pure gluodynamics case, by means of the non-forward gluon distribution, or generalised momentum density, $M\left(x_{1}, x_{2}\right)=M\left(x_{2}, x_{1}\right)$ [188], where $x_{1}, x_{2}$, defined as in Sec. 4.3.3, are suitable for expressing the simplest symmetry properties, following from T-invariance. The $Q^{2}$ evolution in the DGLAP region $x_{1}, x_{2}>0$, which is the only region where positivity constraints are applicable, is given by the following equation

$$
\begin{aligned}
& \frac{\mathrm{d} M\left(x_{1}, x_{2}\right)}{\mathrm{d} t}=\frac{\alpha_{s}}{2 \pi}\left[\int_{x_{1}}^{1} \frac{\mathrm{d} z}{z(1-z)} \tilde{P}\left(z, z^{\prime}\right) M\left(x_{1} / z, x_{2} / z^{\prime}\right)\right. \\
& \left.\quad-\frac{M\left(x_{1}, x_{2}\right)}{2}\left(\int_{0}^{1} \frac{\mathrm{d} z}{1-z} \tilde{P}(z)+\int_{0}^{1} \frac{\mathrm{d} z^{\prime}}{1-z^{\prime}} \tilde{P}\left(z^{\prime}\right)\right)\right]
\end{aligned}
$$

where $t=\ln Q^{2}, \tilde{P}\left(z, z^{\prime}\right)$ and $\tilde{P}(z), \tilde{P}\left(z^{\prime}\right)$ are related to the off-diagonal and diagonal splitting functions $P\left(z, z^{\prime}\right), P(z), P\left(z^{\prime}\right)$ in the following ways: $\tilde{P}\left(z, z^{\prime}\right)=\tilde{P}\left(z^{\prime}, z\right)=z^{\prime}(1-z) P\left(z, z^{\prime}\right)$ implying a similar relation for diagonal kernels $\tilde{P}(z, z)=\tilde{P}(z)=z(1-z) P(z)$. The above evolution equation preserves the symmetry with respect to the interchange of $x_{1}$ and $x_{2}$, because of the simple relation between the integration variables $z$ and $z^{\prime}$ implied by the $t$-channel momentum conservation

$$
\frac{1-z}{z} \frac{1-x_{2}}{x_{2}}=\frac{1-z^{\prime}}{z^{\prime}} \frac{1-x_{1}}{x_{1}}
$$

The above evolution equation allows one to prove the stability of the positivity constraint against $Q^{2}$ evolution, similarly to the quark case, following the general line of [236,237]. To do so, one may 
consider the positive quantities (at some initial scale $\left.Q_{0}\right) M_{ \pm}\left(x_{1}, x_{2}\right)=a M\left(x_{1}\right)+M\left(x_{2}\right) / a \pm$ $2 M\left(x_{1}, x_{2}\right)$, where $a$ is an arbitrary positive number.

The inequality

$$
\left|M\left(x_{1}, x_{2}\right)\right| \leq \sqrt{M\left(x_{1}\right) M\left(x_{2}\right)},
$$

is than the result of the minimization with respect to the variation of $a$, where $M(x)=x g(x)$ is a diagonal momentum distribution. One may write the evolution equations as

$$
\begin{aligned}
\frac{\mathrm{d} M_{ \pm}\left(x_{1}, x_{2}\right)}{\mathrm{d} t}=\frac{\alpha_{s}}{2 \pi}[ & \int_{x_{1}}^{1} \frac{\mathrm{d} z}{z(1-z)}\left(a \tilde{P}(z) M\left(x_{1} / z\right)+\tilde{P}\left(z^{\prime}\right) M\left(x_{2} / z^{\prime}\right) / a\right. \\
& \left.\left. \pm 2 \tilde{P}\left(z, z^{\prime}\right) M\left(x_{1} / z, x_{2} / z^{\prime}\right)\right)-M_{ \pm}\left(x_{1}, x_{2}\right) \int_{0}^{1} \frac{\mathrm{d} z}{1-z} \tilde{P}(z)\right] .
\end{aligned}
$$

It is very important that the virtual contributions are diagonal in the index \pm , so that they cannot change the positivity of the distribution.

To prove positivity of the real term it is sufficient to consider the minimisation with respect to the variation of $z$-dependent (positive) $a(z)$, which can only make the sum of two positive diagonal terms smaller, than in the actual case of minimisation with respect to constant $a$ :

$$
\begin{aligned}
& \min _{a} \int_{x_{1}}^{1} \frac{\mathrm{d} z}{z(1-z)}\left[a \tilde{P}(z) M\left(x_{1} / z\right)+\frac{\tilde{P}\left(z^{\prime}\right)}{a} M\left(x_{2} / z^{\prime}\right) \pm 2 \tilde{P}\left(z, z^{\prime}\right) M\left(x_{1} / z, x_{2} / z^{\prime}\right)\right] \geq \\
& \int_{x_{1}}^{1} \frac{\mathrm{d} z}{z(1-z)} \min _{a(z)}\left[a(z) \tilde{P}(z) M\left(x_{1} / z\right)+\frac{\tilde{P}\left(z^{\prime}\right)}{a(z)} M\left(x_{2} / z^{\prime}\right) \pm 2 \tilde{P}\left(z, z^{\prime}\right) M\left(x_{1} / z, x_{2} / z^{\prime}\right)\right] \\
& \quad=2 \int_{x_{1}}^{1} \frac{\mathrm{d} z}{z(1-z)}\left[\sqrt{\tilde{P}(z) M\left(x_{1} / z\right) \tilde{P}\left(z^{\prime}\right) M\left(x_{2} / z^{\prime}\right)} \pm \tilde{P}\left(z, z^{\prime}\right) M\left(x_{1} / z, x_{2} / z^{\prime}\right)\right] .
\end{aligned}
$$

Writing down Eq. (4.116) for $x_{1} \rightarrow x_{1} / z, x_{2} \rightarrow x_{2} / z^{\prime}$, the sufficient condition of positivity of (4.118) can be easily found [220], namely

$$
\left|\tilde{P}\left(z, z^{\prime}\right)\right| \leq \sqrt{\tilde{P}(z) \tilde{P}\left(z^{\prime}\right)} .
$$

Such inequality is really valid [220] for all $z, z^{\prime}$, completing the proof of positivity. Although we have considered here a pure gluodynamics, the mixing is improving the situation with positivity, providing extra positive terms, like in the forward case [237].

v) - Preservation of positivity for the BFKL equation Let us now move to the positivity properties which can be currently established only at the perturbative level. Since the non-perturbative counterpart of BFKL evolution, namely, the hadron impact factor, is not expressed in the form of a matrix element, the proof of positivity by the methods of Sec. 4.3 is not possible. The BFKL evolution, as it will be discussed, may be presented in a form of generalised master equation, so that the gain and loss probabilities differ,

$$
\frac{d f(x, t)}{d t}=\int \mathrm{d} y\left[w_{+}(y \rightarrow x) f(y, t)-w_{-}(x \rightarrow y) f(x, t)\right] .
$$

It will preserve positivity as long as one considers the evolution towards larger longitudinal "time" $t=\ln (1 / x)$, that is towards smaller partonic momentum fractions $x$. Note that in the subsequent expressions of this section, $x=\ln k_{T}$ is used to denote the variable which is the transverse coordinate in the BFKL equation ${ }^{26}$.

\footnotetext{
${ }^{26}$ Note the difference with the DGLAP definitions of $t$ and $x$. These notations will be used only throughout this paragraph.
} 
Moreover, the extra non-linear negative term [254], responsible for gluons fusion will not violate this property. The specific property of generalised master equation is the different values of gain $w_{+}$ and loss $w_{-}$probabilities. Therefore, it describes not only transitions of "particles" but also their fission (multiplication), when the gain probability prevails, as in the case of the BFKL equation, or fusion in the opposite case. However, this does not affect the proof of positivity, which in fact is making use only of the positivity of $w_{+}$and locality of loss term due to $w_{-}$. The non linearity of the loss term also does not spoil the proof of positivity, as soon as it remains local.

In order to separate the effects of diffusion and fission, one may consider [238] the master equation for the weighted function $f_{\sigma}(x, t)=f(x, t) \sigma(x)$, so that $w_{+}(x, y) \rightarrow w_{\sigma}(x, y)=$ $w_{+}(x, y) \sigma(x) / \sigma(y)$, while $w_{-}$remains unchanged. For the BFKL case, the function $\sigma(x)$ can be simply $\left(k_{T}\right)^{\alpha}$ with $0<\alpha<1$, in order to avoid divergences. Let us also define the "relative" function as $\bar{f}_{\sigma}(x, t)=f_{\sigma}(x, t) /\left\langle f_{\sigma}(t)\right\rangle$, with the $k_{T}$-integrated function $\left\langle f_{\sigma}(t)\right\rangle=\int d x f_{\sigma}(x, t)$. It satisfies the standard master equation

$$
\frac{\mathrm{d} \bar{f}_{\sigma}(x, t)}{\mathrm{d} t}=\int \mathrm{d} y\left[w_{\sigma}(y \rightarrow x) f_{\sigma}(y)-w_{\sigma}(x \rightarrow y) \bar{f}_{\sigma}(x)\right] .
$$

If $\left\langle f_{\sigma}(t)\right\rangle$ evolves exponentially, as $\mathrm{d}\left\langle f_{\sigma}(t)\right\rangle / \mathrm{d} t=\lambda_{\sigma}\left\langle f_{\sigma}(t)\right\rangle, \lambda_{\sigma}$ is solution of an eigenvalue problem defining simultaneously the eigenfunction $\sigma$

$$
\int \mathrm{d} x w_{+}(y \rightarrow x) \sigma(x)=\left[\lambda_{\sigma}+\int \mathrm{d} x w_{-}(y \rightarrow x)\right] \sigma(y),
$$

and we have

$$
\lambda_{\sigma}=\int \mathrm{d} x\left[w_{\sigma}(y \rightarrow x)-w_{-}(y \rightarrow x)\right]
$$

which is independent of $y$.

It turns out that the separation of these effects for the BFKL equation is ambiguous, due to the freedom in the choice of the function $\sigma$, reflecting the scale invariance of gain and loss probabilities, while, when they are not separated, their combination is invariant [238]. This invariance in the short-range approximation leads to

$$
\lambda_{0}=\lambda_{\sigma}-\frac{v_{\sigma}^{2}}{4 D_{\sigma}} .
$$

where $\lambda_{0}$ is independent of $\sigma$. In fact, it is just this combination which gives the famous BFKL value for the Pomeron intercept, proportional to $4 \ln 2$, corresponding to the minimal fission and diffusion and to the absence of drift in this approach.

vi) - Convexity properties of parton distributions Another property which can be established at the perturbative level is the stability in $t$ of the signs of the derivative with respect to $x$ of the parton distribution. It follows [255] from the differential form (4.105) in which the operator in the r.h.s. commutes with the (logarithmic) derivative in $x$. This immediately means that the derivatives obey the same evolution equation. Therefore, their sign is also preserved by the evolution. One has the pattern of alternate signs for the derivatives of increasing order.

This may explain the success of a simple concave parametrisation of $x^{-a}(1-x)^{b}$ type. It is interesting, that if one uses the parton distributions as an input to construct a model for GPD, the concave behaviour guarantees [256] the validity of the positivity constraints for the GPD (see Sec. 4.3.3).

This concave parametrisation explains why the results which are, strictly speaking, valid at very small $x$, still holds at much larger $x$, if the factor $(1-x)^{b}$ is still close to 1 . Such a situation happened [257] when the behaviour of neutron spin-dependent structure function $g_{1}$ at SLAC energies was found to be compatible with the perturbative low- $x$ asymptotics (see [258] and references therein). Repeating this argumentation for spin-averaged case one may expect the applicability of 
BFKL approach at larger $x$ as well, because the BFKL equation (as well as its non-linear generalisations) also preserves the concavity of (unintegrated) gluon distribution in $x$, as soon as the gain and loss probabilities in the generalised master equation do not depend on the longitudinal momentum fraction $x$. The observation [259] on its violation may be considered as a signal of a different kind of evolution at very small $x$. At the same time, another recent analysis [260] shows an agreement with the predicted concave behaviour.

vii) - Irreversibility: longitudinal and transverse scale arrows The positivity property is preserved when the respective "time" variable in the master equation is increasing, but it may be violated for the backward evolution. This defines a "scale arrow" analogous to the famous time's arrow. The reason for such irreversibility in positivity preservation is the following. The master equation clearly distinguishes the directions of time, in other words time reflection makes it "antiprobabilistic". To understand the general origin of this distinction, let us start, following G.M. Zaslavsky [261], from the reversible Kolmogorov-Chapman equation

$$
W\left(x_{1}, t_{1} \mid x_{2}, t_{2}\right)=\int \mathrm{d} y W\left(x_{1}, t_{1} \mid y, \tau\right) W\left(y, \tau \mid x_{2}, t_{2}\right),
$$

with a probability depending only on $t_{1}-t_{2}{ }^{27}$

$$
W\left(x_{1}, x_{2}, t\right)=\int \mathrm{d} y W\left(x_{1}, \mid y, t-\tau\right) W\left(x_{2}, y, \tau\right),
$$

$\tau \in\left[t_{1}, t_{2}\right]$ being any intermediate time. Let us write the following expression for the difference

$$
W(x, \xi, t+\delta t)-W(x, \xi, t)=\int \mathrm{d} y[W(x, y, \delta t) W(y, \xi, t)-W(y, x, \delta t) W(x, \xi, t)],
$$

where we use the completeness condition

$$
\int \mathrm{d} y W(y, x, \delta t)=1
$$

Note that Eq. (4.127) is already a master equation, in a more general form, with finite difference instead of derivative corresponding to the special case $\delta t \rightarrow 0$. The probability $W(y, \xi, t)$ depending on the initial value $\xi$ should be considered as a distribution function, while $W(x, y, \delta t)$ is the transition probability. It is not necessary, although possible, to integrate over the initial value $\xi$ or to "forget" it. Its very existence in past is sufficient. Choosing the value $\xi$ in future, one immediately comes to the "antiprobabilistic" master equation. The short-range limits for these two cases correspond to two Kolmogorov equations, the first one coinciding with the Fokker-Planck equation.

These two points mark the appearance of the time ordering at the earlier stage of comparison with Ref. [261]. It is possible, following this reference, to consider the limit of small $\delta t$ and to define the differential probability rate (which can be also fractional [261]), resulting in the standard differential form of master equation. Note finally, that to manifest irreversibility, the transition probability should also not be reversible in the sense that the transition from different points to a single one should be allowed. This is an analogue of the coarse-graining procedure which is a well-known ingredient of the emergence of irreversibility. This property is obviously valid in the case of QCD evolution equations, where a corresponding scale arrow arises.

The striking difference between the BFKL and DGLAP equations is that the "time" (whose role is played by scale) direction is pointed to the infrared (IR) in the BFKL case, and to the ultraviolet (UV) in the DGLAP one. The IR direction is quite natural for the Wilson renormalisation group (RG) and, indeed, there are studies [254] where BFKL dynamics emerges within the Wilson RG approach.

\footnotetext{
${ }^{27}$ It corresponds in QCD to the choice of the variable $t=\ln k_{T}$ for the DGLAP equation and of $t=-\ln x$ for the BFKL equation.
} 
Note also that for the DGLAP equation, the Wilson RG may be considered in momentum, rather than in coordinate space. Indeed, the parton distributions may be described as integrated over transverse momentum up to the actual energy scale $Q^{2}$

$$
F\left(x, Q^{2}\right)=\int_{0}^{Q^{2}} \mathrm{~d} k_{T}^{2} f\left(x, k_{T}^{2}\right) .
$$

Note that this equation is compatible with the probabilistic properties of the integrand only when the 1.h.s is increasing with $Q^{2}$, i.e., for small enough $x$. The different directions of longitudinal and transverse scale arrows may be unified by assuming the fundamental role of an angular ordering, related to QCD coherence equations [262-265]. If so, its projection onto longitudinal direction provides the IR longitudinal scale arrow, while its projection to transverse direction results in UV transverse scale arrow.

Let us compare the role of irreversibility for distribution and fragmentation functions. The evolution of the latter is also described by the DGLAP equation. The time development of the hard scattering process corresponds to the probabilistic evolution of distributions and antiprobabilistic evolution of fragmentation functions. The latter property may be important for the explanation of the strong dependence of the final state characteristics on the initial conditions, and for the possible emergence of turbulence-like phenomena. 


\section{Further developments}

\subsection{Polarised cascades}

\subsubsection{Dual types of density matrices}

In Eq. (2.64) we have introduced the acceptance matrix $\check{\rho}$ to characterise a detector. For a spin one-half,

$$
\check{\rho}=\epsilon_{0}(1+\boldsymbol{A} \cdot \boldsymbol{\sigma}) .
$$

$\epsilon_{0}$ is the unpolarised efficiency and $\boldsymbol{A}$ is the vector analysing power. $\check{\rho}$ has the same properties as a density matrix, except for the trace condition. More generally an acceptance matrix is attached to any process that the particle may undergo in the future, for instance scattering, decay, absorption or detection. The probability that a particle of density matrix $\hat{\rho}$ undergoes such a process is given by

$$
w \propto \operatorname{Tr}(\hat{\rho} \check{\rho}) .
$$

For spin one-half, $\hat{\rho}=(\mathbb{1}+\boldsymbol{S} . \boldsymbol{\sigma}) / 2$, then

$$
w=\epsilon_{0}(1+\boldsymbol{S} \cdot \boldsymbol{A})
$$

As opposed to $\check{\rho}$, the density matrix $\hat{\rho}$ is the result of the past interactions and is an emittance matrix. More generally, emittance matrices can describe intensity-like quantities, like the spin-dependent quark distribution, in which case their traces are not normalised to unity. An acceptance matrix can be seen as an emittance matrix in the time-reversed process. The cross section matrix is ambivalent: it acts as an acceptance matrix for the initial particles and as an emittance matrix for the final ones. An example where emittance and acceptance matrices are implicitly used is in the expression $l^{\mu \nu} W_{\mu \nu}$ for the cross section of deep inelastic electron scattering. It is of the form $\operatorname{Tr}(\hat{\rho} \check{\rho})$ with $\hat{\rho}=l^{\mu \nu}$ and $\check{\rho}=W^{\mu \nu}$ (or vice-versa, depending on the chosen arrow of the virtual photon line).

\subsubsection{Monte-Carlo simulations of entangled cascades}

Suppose, for instance, that one needs a Monte-Carlo simulation of the cascade of reactions

$$
\begin{aligned}
\mathrm{e}^{+} \mathrm{e}^{-} & \rightarrow \tau^{+} \tau^{-}, \\
\tau^{+} & \rightarrow \mu^{+} \nu_{\mu} \bar{\nu}_{\tau}, \\
\tau^{-} & \rightarrow \mu^{-} \bar{\nu}_{\mu} \nu_{\tau} .
\end{aligned}
$$

Can the event generator treat the different reactions (5.4) successively? Even if we are not interested in the muon polarisations, the generator has to include the spin degree of freedom of the $\tau$ leptons. Polarised $\tau$ have, indeed, anisotropic decays of the form $1+A \boldsymbol{S} . \hat{\boldsymbol{p}}$ and $1-A \boldsymbol{S}^{\prime} \cdot \hat{\boldsymbol{p}}^{\prime}$, where $\boldsymbol{S}$ and $\boldsymbol{S}^{\prime}$ are the $\tau^{ \pm}$polarisations and $\hat{\boldsymbol{p}}$ and $\hat{\boldsymbol{p}}^{\prime}$ the muon directions in the $\tau^{ \pm}$rest frames. A simple MonteCarlo program would first generate the $\tau^{+}$and $\tau^{-}$momenta, calculate their individual polarisations $\boldsymbol{S}$ and $\boldsymbol{S}^{\prime}$ (which are nonzero due to the contribution of $Z^{0}$ exchange), then simulate the $\tau^{+}$and $\tau^{-}$ anisotropic decays independently. Such a program would correctly give the individual muon spectra, but would miss the influence of the $\tau^{+} \tau^{-}$spin correlations on the joint momentum distribution of the two muons. As a remedy, one might try to generate correlated polarisation vectors $\boldsymbol{S}$ and $\boldsymbol{S}^{\prime}$ at the $\tau^{+} \tau^{-}$production vertex, with a joint distribution $C\left(\boldsymbol{S}, \boldsymbol{S}^{\prime}\right)$. But whichever the latter may be, it would only describe a classical correlation, corresponding to the separable $\tau^{+} \tau^{-}$density matrix

$$
\rho_{\mathrm{sep}}=\int \frac{\mathrm{d}^{2} \boldsymbol{S}}{4 \pi} \frac{\mathrm{d}^{2} \boldsymbol{S}^{\prime}}{4 \pi} C\left(\boldsymbol{S}, \boldsymbol{S}^{\prime}\right) \frac{\mathbb{1}+\boldsymbol{S} \cdot \boldsymbol{\sigma}}{2} \otimes \frac{\mathbb{1}+\boldsymbol{S}^{\prime} \cdot \boldsymbol{\sigma}^{\prime}}{2},
$$

whereas the actual $\tau^{+} \tau^{-}$spin correlation is often of the entangled type. For instance, at $90^{\circ}$, onephoton exchange produces the $\tau^{+} \tau^{-}$pair in the spin-triplet state corresponding to $\left\langle\boldsymbol{\sigma}+\boldsymbol{\sigma}^{\prime}\right\rangle . \boldsymbol{n}=0$ 
and described by the density matrix $\rho=\left(\mathbb{1}-\sigma \cdot R \boldsymbol{\sigma}^{\prime}\right) / 4$, where $R$ is the symmetry about the $\boldsymbol{n}$ axis. With the convention of Fig.1, it means $C_{l l}=C_{m m}=C_{n n}=-C_{00} \equiv-1$ and $C_{\mu \nu}=0$ for $\mu \neq \nu$. This is a maximally entangled state. The strongest classical correlation of the form $C_{i j} \propto \delta_{i j}, C_{i 0}=C_{0 i}=0$, that is to say, $\boldsymbol{S}=-R \boldsymbol{S}^{\prime}$, is given by $C\left(\boldsymbol{S}, \boldsymbol{S}^{\prime}\right)=\delta^{2}\left(\boldsymbol{S}+R \boldsymbol{S}^{\prime}\right)$. It leads to $\rho_{\mathrm{sep}}=\left[\mathbb{1}-\left(\boldsymbol{\sigma} \cdot R \boldsymbol{\sigma}^{\prime}\right) / 3\right] / 4$, which represents a correlation three time too weak.

A correct Monte-Carlo algorithm, taking entanglement into account, has been proposed by Collins and Knowles [266, 267] (see also [268]). Here we show how it applies to the reaction (5.4). To treat the spin in the various subprocesses of (5.4), it is convenient to use the partially transposed cross section matrix $\widetilde{\mathcal{R}}$ defined in (2.68) or a reduced form obtained by partial trace, like in (2.74). More particularly, one needs the expressions of the matrices $\widetilde{\mathcal{R}}_{1}(\theta, \varphi), \widetilde{\mathcal{R}}_{2}\left(p_{\tau}, p_{\mu}\right)$, and $\widetilde{\mathcal{R}}_{3}\left(p_{\tau}^{-}, p_{\mu}^{-}\right)$ of the subprocesses (1-3) in function of their kinematical variables. Since one is only interested in the muon momenta, the summation is made over the final polarisations in $\widetilde{\mathcal{R}}_{2}$ and $\widetilde{\mathcal{R}}_{3}$ and the integration is made over the (unobservable) $\nu_{\mu}-\bar{\nu}_{\tau}$ and $\bar{\nu}_{\mu}-\nu_{\tau}$ relative momenta. The initial electrons may be polarised, described by density matrices $\rho\left(\mathrm{e}^{+}\right)$and $\rho\left(\mathrm{e}^{-}\right)$. To lighten the equations, the density matrices will not be explicitly normalised. The event generation for the full reaction (5.4) proceeds in the following steps:

- generate the momenta $p_{\tau}^{+}$and $p_{\tau}^{-}$(i.e., $\theta$ and $\varphi$ ) according to the differential cross section

$$
\frac{d \sigma}{d \Omega}\left(\overrightarrow{\mathrm{e}^{+}} \overrightarrow{\mathrm{e}^{-}} \rightarrow \tau^{+} \tau^{-}\right)=\left\langle a\left|\rho\left(\mathrm{e}^{+}\right)\right| a^{\prime}\right\rangle\left\langle b\left|\rho\left(\mathrm{e}^{-}\right)\right| b^{\prime}\right\rangle\left\langle a^{\prime}, b^{\prime} ; c, d\left|\widetilde{\mathcal{R}}_{1}(\theta, \varphi)\right| a, b, c, d\right\rangle
$$

(summation over repeated indices is understood),

- keeping these momenta, calculate the (emittance-type) density matrix of $\tau^{+}$

$$
\left\langle c\left|\rho\left(\tau^{+}\right)\right| c^{\prime}\right\rangle \propto\left\langle a\left|\rho\left(\mathrm{e}^{+}\right)\right| a^{\prime}\right\rangle\left\langle b\left|\rho\left(\mathrm{e}^{-}\right)\right| b^{\prime}\right\rangle\left\langle a^{\prime}, b^{\prime} ; c, d\left|\widetilde{\mathcal{R}}_{1}(\theta, \varphi)\right| a, b, c^{\prime}, d\right\rangle,
$$

- generate the $\mu^{+}$momentum according to the decay distribution

$$
\left\langle c\left|\rho\left(\tau^{+}\right)\right| c^{\prime}\right\rangle\left\langle c^{\prime}\left|\widetilde{\mathcal{R}}_{2}\left(p_{\tau}^{+}, p_{\mu}^{+}\right)\right| c\right\rangle,
$$

- keeping this momentum, calculate the $\tau^{+}$acceptance matrix

$$
\left\langle c^{\prime}\left|\check{\rho}\left(\tau^{+}\right)\right| c\right\rangle \propto\left\langle c^{\prime}\left|\widetilde{\mathcal{R}}_{2}\left(p_{\tau}^{+}, p_{\mu}^{+}\right)\right| c\right\rangle,
$$

- going back to the first reaction, calculate the (emittance-type) density matrix of $\tau^{-}$

$$
\left\langle d\left|\rho\left(\tau^{-}\right)\right| d^{\prime}\right\rangle \propto\left\langle a\left|\rho\left(\mathrm{e}^{+}\right)\right| a^{\prime}\right\rangle\left\langle b\left|\rho\left(\mathrm{e}^{-}\right)\right| b^{\prime}\right\rangle\left\langle c^{\prime}\left|\check{\rho}\left(\tau^{+}\right)\right| c\right\rangle\left\langle a^{\prime}, b^{\prime} ; c, d\left|\widetilde{\mathcal{R}}_{1}(\theta, \varphi)\right| a, b, c^{\prime}, d^{\prime}\right\rangle,
$$

- generate the $\mu^{-}$momentum according to the decay distribution

$$
\left\langle d\left|\rho\left(\tau^{-}\right)\right| d^{\prime}\right\rangle\left\langle d^{\prime}\left|\widetilde{\mathcal{R}}_{3}\left(p_{\tau}^{-}, p_{\mu}^{-}\right)\right| d\right\rangle .
$$

The different steps of the simulation are schematised in Fig. 37-a).

This method can be generalised to any process described by tree graphs: cascade decays, QCD jets, electromagnetic showers, etc., when one needs the momentum or spin correlations between particles which are on different branches of the tree. This is illustrated in Fig. 37-b).

\subsection{Quantum information aspects}

The present report brings out the role of vector of information played by the spin. Polarised scattering experiments make use of this vector to extract the information on 

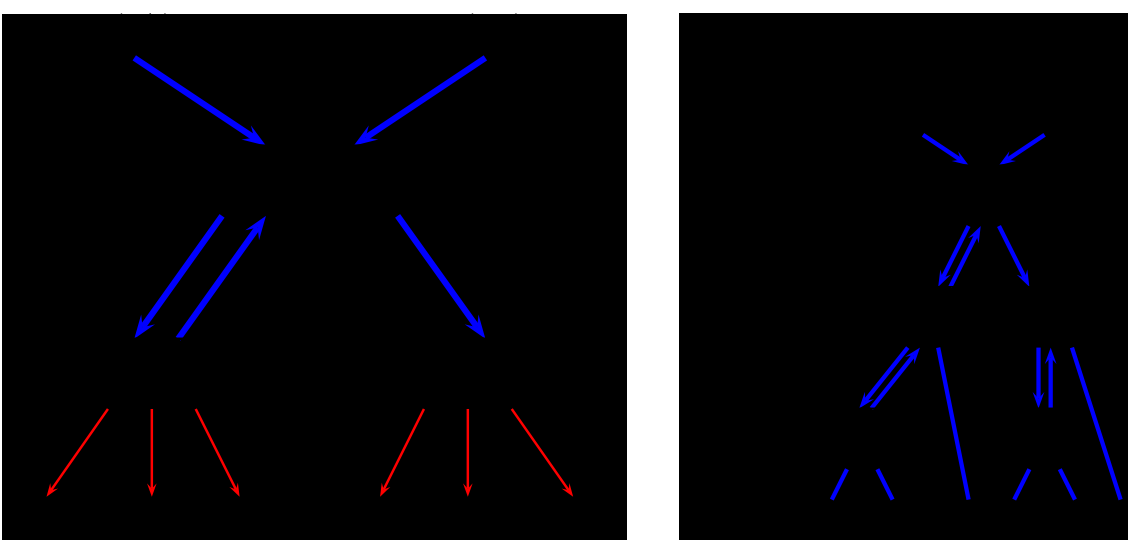

Figure 37: Successive steps of the Monte-Carlo algorithm generating momentum correlations in cascade reactions. a) reactions $\mathrm{e}^{+} \mathrm{e}^{-} \rightarrow \tau^{+} \tau^{-}, \tau^{+} \rightarrow \mu^{+} \nu_{\mu} \bar{\nu}_{\tau}$ and $\tau^{-} \rightarrow \mu^{-} \bar{\nu}_{\mu} \nu_{\tau}$; b) a more complicated tree diagram. Downward arrows represent density matrices, upward arrows represent acceptance matrices.

- a physical state, e.g., the internal structure of nucleons or the spin-parity of a new particle. The information is encoded in a density matrix $\rho$.

- an interaction mechanism, e.g., a Reggeon exchange or a non-minimal coupling. The information is encoded in a cross section matrix $\mathcal{R}$.

In this subsection we give some qualitative and quantitive laws governing the efficiency of the polarised experiment in extracting the desired information, taking into account its quantum aspects which have been briefly presented in Secs. 2.2 and 2.4.

\subsubsection{Various kinds of entropy}

We recall that a pure state of a quantum system is a statistical state on which one has the maximum information. Its density matrix is of the form $\rho=|\psi\rangle\langle\psi|$ and has rank 1 . The opposite case (no information at all) is given by the matrix $\rho_{0}=\mathbb{1}_{N} / N$ of rank $N$, where $\mathbb{1}_{N}$ is the unit $N \times N$ matrix and $N$ the number of basic states. Between these two cases, one can quantify the lack of information about the system by various kinds of entropy (see, for instance, [269]), among which the Shannon entropy

$$
E^{(1)}(\rho)=-\operatorname{Tr}\{\rho \log \rho\},
$$

and the Rényi entropy of order $q$

$$
E^{(q)}(\rho)=\frac{\ln \operatorname{Tr} \rho^{q}}{1-q} \quad(0 \leq q \neq 1),
$$

which converges to the Shannon entropy for $q \rightarrow 1$. In the limiting case $q=0$ and $q=+\infty$, we have respectively $E^{(0)}(\rho)=\ln [\operatorname{rank}(\rho)]$ and $E^{(\infty)}(\rho)=-\ln \left(w_{\max }\right)$, where $w_{\max }$ is the largest eigenvalue of $\rho$. Roughly speaking, for small $q, E^{(q)}(\rho)$ is an "egalitarian" measure of entropy (all nonzero eigenvalues count more or less equally), whereas for large $q, E^{(q)}(\rho)$ is an "elitist" measure (only the largest eigenvalues count).

The Shannon entropy has the following properties

1. $0 \leq E(\rho) \leq \ln N$. The left equality is obtained for pure states and the right one for $\rho=\rho_{0}$. 
2. For a mixing $\rho=\lambda \rho_{1}+\mu \rho_{2}$ with $0 \leq \lambda=1-\mu \leq 1$, one has $\lambda E_{1}+\mu E_{2} \leq E \leq \lambda E_{1}+\mu E_{2}+E_{\text {mixing }}$, where $E_{i} \equiv E\left(\rho_{i}\right)$ and $\rho_{\text {mixing }}=\left(\begin{array}{cc}\lambda & 0 \\ 0 & \mu\end{array}\right)$.

3. Removing the non-diagonal elements of a density matrix increases its entropy.

4. For a composite system $C=A+B$, one has the "triangle inequality" $\left|E_{A}-E_{B}\right| \leq E_{C} \leq$ $E_{A}+E_{B}$, where $E_{i} \equiv E\left(\rho_{i}\right)$ and $\rho_{A}$ and $\rho_{B}$ are the individual density matrices of $A$ and $B$, given by the partial traces $\rho_{A}=\operatorname{Tr}_{B}\left(\rho_{C}\right)$ and $\rho_{B}=\operatorname{Tr}_{A}\left(\rho_{C}\right)$.

5. $E_{C}=E_{A}+E_{B}$ if $A$ and $B$ are uncorrelated, i.e., $\rho_{C}=\rho_{A} \otimes \rho_{B}$.

6. If $C$ is a separable pure state, i.e., $\rho_{C}=|\psi\rangle\langle\psi|$ with $|\psi\rangle=\left|\psi_{A}\right\rangle \otimes\left|\psi_{B}\right\rangle$, then $E_{A}=E_{B}=0$.

7. If $C$ is an entangled pure state, $E_{A}=E_{B}>0$.

For Rényi entropies with $q \neq 1$, properties $1,5,6,7$ are still valid. The mixing property 2 is replaced by the weaker one

$$
E \geq \inf \left\{E_{1}, E_{2}\right\}
$$

which implies that the density matrices of entropy greater than some fixed value $E_{m}$ form a convex subset, containing $\rho_{0}$, of the positivity domain. More properties are given in [270].

Other types of entropies can be defined. We will generalise the name of entropy to any symmetric functions of the eigenvalues of $\rho$ satisfying (5.14), i.e., which can only increase upon mixing. This is the case for $\Delta_{2}, \Delta_{3}, \ldots \Delta_{n}$ of 2.11 and for the distance to the boundary of the positivity domain. The various entropies are not independent, since they are functions of the $n$ quantities $\Delta_{i}$ 's.

\subsubsection{Entropy of the cross section matrix}

Let us consider, for definiteness, the scattering experiment $A+B \rightarrow C+D(+X)$, where $X$ is the set of non-analysed or undetected particles. The initial spins are generally uncorrelated: $\rho_{A+B}=$ $\rho_{A} \otimes \rho_{B}$, therefore the initial entropy is $E_{A+B}=E_{A}+E_{B}$ and its ranks is $r_{A+B}=r_{A} r_{B}$.

Let us first treat the completely polarised exclusive experiment (no $X$ ). If the initial particles are fully polarised, both initial and final states are pure: $\rho_{A+B}=\left|\Psi_{A+B}\right\rangle\left\langle\Psi_{A+B}\right|$ with $\left|\Psi_{A+B}\right\rangle=$ $\left|\Psi_{A}\right\rangle \otimes\left|\Psi_{B}\right\rangle$ and $\rho_{C+D}=\left|\Psi_{C+D}\right\rangle\left\langle\Psi_{C+D}\right|$ with $\left|\Psi_{C+D}\right\rangle=\mathcal{M}\left|\Psi_{A+B}\right\rangle$ and $r_{A+B}=r_{C+D}=1$. However, $C$ and $D$, taken separately, are only partially polarised if $\left|\Psi_{C+D}\right\rangle$ is an entangled state, which is the most general case. If $A$ and $B$ are partially polarised (mixed $A+B$ state, $r_{A+B}>1$ ), the $C+D$ state can be pure or mixed with $r_{C+D} \leq \min \left\{r_{A+B}, r_{\mathcal{M}}\right\}, r_{\mathcal{M}}$ being the rank of the transition matrix. Examples of $r_{C+D}<r_{A+B}$ are $\mathrm{e}^{+} \mathrm{e}^{-} \rightarrow \nu \bar{\nu}$ and $\gamma \gamma \rightarrow \pi^{0} \rightarrow \mathrm{e}^{+} \mathrm{e}^{-}$. In these two cases $r_{\mathcal{M}}=1$ and $r_{C+D}=1$, even if the initial particles are unpolarised $\left(r_{A+B}=4\right)$.

Let us now consider the inclusive case $(X \neq \varnothing)$. Instead of $\mathcal{M}$ we use the inclusive cross section matrix $\mathcal{R}$ defined in Sec. 2.5.2 or its partial transpose $\widetilde{\mathcal{R}}$. The density matrix of $C+D$ as a function of $\rho_{A+B}$ is given, according to 2.69 , by

$$
\rho_{C+D}\left(\rho_{A+B}\right)=\operatorname{Tr}_{A, B}\left(\widetilde{\mathcal{R}} \rho_{A+B}\right) / \operatorname{Tr}\left(\widetilde{\mathcal{R}} \rho_{A+B}\right) .
$$

One can introduce the entropy $E(\mathcal{R})$ of the CSM, replacing $\rho$ in (5.12-5.13) by the unit trace matrix $\mathcal{R} / \operatorname{Tr} \mathcal{R}$. This entropy comes from the leakage of information toward $X$. We may call it entropy of inclusiveness ${ }^{28}$. The rank $r(\mathcal{R})$ of $\mathcal{R}$ cannot exceed the dimension $d(X)$ of the Hilbert space of $X$ (which is infinite if $X$ contains several particles, due to their continuous relative momenta). The former case of completely polarised exclusive reaction can be treated as a special case with $X=\varnothing$, $r(\mathcal{R})=d(X)=1$ and $E(\mathcal{R})=0$. It is to be expected that the CSM of an inclusive reaction with high missing mass has a large entropy, manifested in spin observables being well below the positivity bounds. This should apply to the Soffer bound at small $x$, corresponding to a high mass of the spectator system, as mentionned in Sec. 4.3.2.

\footnotetext{
${ }^{28}$ For a reduced CSM like $\mathcal{R}_{A, C+D}$, part of this entropy also comes from the unpolarised $B$ state.
} 


\subsubsection{Factorisation, separability or entanglement of the cross section matrix}

The CSM may be factorised in an initial state acceptance matrix and a final state emittance matrix (transposed): $\mathcal{R}=\check{\rho}_{A+B} \otimes \rho_{C+D}^{t}$. This is the case when the reaction is strongly dominated by a spin-zero exchange in the $s$-channel, as in $\gamma \gamma \rightarrow \pi^{0} \rightarrow \mathrm{e}^{+} \mathrm{e}^{-}$. The factorisation of $\mathcal{R}$ comes from the factorisation of the amplitude $\mathcal{M}=|\Psi\rangle\langle\Phi|$. No spin information is transmitted between the initial and the final state. More generally, the CSM may be "initial-final separable":

$$
\mathcal{R}=\sum_{n} w_{n} \check{\rho}_{n, A+B} \otimes \rho_{n, C+D}^{t}, \quad w_{n}>0,
$$

in analogy with Eq. (2.57). Then $\widetilde{\mathcal{R}}=\sum w_{n} \check{\rho}_{n, A+B} \otimes \rho_{n, C+D}$ is also positive and separable and the final density matrix is given in terms of the initial one by

$$
\rho_{C+D}\left(\rho_{A+B}\right)=\sum w_{n} \operatorname{Tr}\left(\rho_{n, A+B} \check{\rho}_{n, A+B}\right) \rho_{n, C+D} .
$$

If the form (5.16) is not possible, the CSM is "initial-final entangled". For exclusive reactions, if the amplitude is a sum of factorised terms, $\mathcal{M}=\sum\left|\Psi_{n}\right\rangle\left\langle\Phi_{n}\right|$, the interference between these terms gives rise to entanglement between initial and final particles. For inclusive reactions the interference may be washed out when summing over the $X$ states, giving an "initial-final separable" CSM.

Factorisation, separability or entanglement of the CSM may also refer to partitions of the external particles other than initial versus final ones. Many peripheral reactions, like single or double diffraction dissociation, are dominated by the exchange of a particle, Reggeon or Pomeron, here denoted $Q$, in the $t$-channel. They can be decomposed, for instance, in the subreactions $A+Q \rightarrow C$, $X^{\prime}$ or $C+X^{\prime}$ and $B+\bar{Q} \rightarrow D, X^{\prime \prime}$ or $D+X^{\prime \prime}$. Besides, even if $Q$ has nonzero spin, it may be dominantly exchanged in only one spin state, therefore effectively carry no spin information. Examples are the electromagnetic dissociation of relativistic nuclei, which mainly occurs through $S_{z}=0$ photon exchange, and the reaction $\mathrm{e}^{+} \mathrm{e}^{-} \rightarrow W^{+} W^{-}$at $s \gg t, m_{W}^{2}$, where neutrino exchange is dominating. For these reactions the amplitude and the CSM are factorised as follows

$$
\mathcal{M}=\mathcal{M}_{A} F\left(Q^{2}\right) \mathcal{M}_{B}, \quad \mathcal{R}=\left|F\left(Q^{2}\right)\right|^{2} \mathcal{R}_{A, C} \otimes \mathcal{R}_{B, D}
$$

It means no $A-B$ or $C-D$ spin correlation, no $A \rightarrow D$ or $B \rightarrow C$ spin transfer and positivity yields separate conditions for the two sub-reactions. If $X^{\prime}$ is empty, the inequalities for $A+Q \rightarrow C$ are saturated.

\subsubsection{Entropy of the observed final state}

The entropy $E_{C+D}$ of the analysed particles comes partly from $A$ and $B$, partly from $\mathcal{R}$. One can draw the more or less qualitative laws

- $E_{C+D}$ is a non-decreasing function of $E_{A}, E_{B}$ and $E_{\mathcal{R}}$.

- if $E_{A}=E_{B}=E_{\mathcal{R}}=0$, then $E_{C+D}=0$ (case of an exclusive reaction with perfect initial polarisation).

- if $\mathcal{R}$ is "initial-final factorised" (Eq. (5.16) with only one term) then $\rho_{C+D}$ is independent of $\rho_{A}$ and $\rho_{B}$ and one has $E_{C+D}=E_{\mathcal{R}}$. An example is $\overline{\mathrm{p}} \mathrm{p} \rightarrow \eta_{c} \rightarrow \mathrm{K}^{* 0}+\overline{\mathrm{K}}^{* 0}+X$.

- if $R$ is pure (no $X$ ) and "initial-final factorised": $R \propto(|\Phi\rangle\langle\Phi|)_{i} \otimes\left(|\Psi\rangle\langle\Psi||)_{f}^{t}\right.$, then $\rho_{C+D}=$ $|\Psi\rangle\langle\Psi|$ is pure. This is the case of $\overline{\mathrm{p}} \rightarrow \eta_{c} \rightarrow \mathrm{K}^{*}+\overline{\mathrm{K}}^{*}$.

- if $R$ is pure and "initial-final entangled", $E_{C+D}$ may be nonzero. For unpolarised $A$ and $B$ $E_{C+D}$ is a measure of the entanglement of $R$. 


\subsubsection{Transfer of information from initial to final state}

Let us focus on the sensitivity of $\rho_{C+D}$ to $\rho_{A+B}$. To simplify, we will ignore the spins of $B$ and $D$. We will also use the shorter notations $\mathcal{R}$ for the reduced $\operatorname{CSM} \mathcal{R}_{A, C}, \mathcal{A} \equiv \rho_{A}$ and $\mathcal{C}=\operatorname{Tr}_{A}\{\widetilde{\mathcal{R}} A\}$ (wherefrom $\rho_{C}=\mathcal{C} / \operatorname{Tr} \mathcal{C}$ ). The matrix $\mathcal{C}$ contains the same spin information as $\rho_{C}$ and in addition it gives the initially polarised cross section $\sigma\left(\rho_{A}\right) \propto \operatorname{Tr} \mathcal{C}$. To express the linear dependence of $\mathcal{C}$ on $\mathcal{A}$, we unwind these matrices into column vectors of dimension $n_{A}^{2}$ and $n_{C}^{2}$ :

$$
\mathcal{A} \rightarrow \boldsymbol{A}=\left(A_{11}, A_{12}, \ldots A_{1 n_{A}}, A_{21}, A_{22}, \ldots\right)^{t}
$$

and similarly for $C \rightarrow \mathcal{C}$. One thus has two equivalent writings:

$$
\begin{aligned}
& \mathcal{C}=\operatorname{Tr}_{A}(\widetilde{\mathcal{R}} \mathcal{A}) \quad \rightarrow \quad\left\langle j|\mathcal{C}| j^{\prime}\right\rangle=\left\langle i^{\prime} j|\widetilde{\mathcal{R}}| i j^{\prime}\right\rangle\left\langle i|\mathcal{A}| i^{\prime}\right\rangle, \\
& \boldsymbol{C}=J \boldsymbol{A} \quad \rightarrow \quad\left\langle j j^{\prime} \mid \boldsymbol{C}\right\rangle=\left\langle j j^{\prime}|J| i i^{\prime}\right\rangle\left\langle i i^{\prime} \mid \boldsymbol{A}\right\rangle,
\end{aligned}
$$

where we have used the Dirac notation both for the original matrices and the unwound ones, e.g., $\left\langle i i^{\prime} \mid \boldsymbol{A}\right\rangle \equiv A_{i i^{\prime}} \equiv\left\langle i|\mathcal{A}| i^{\prime}\right\rangle$. The matrix $J$ is obtained from $\widetilde{\mathcal{R}}$ by the circular permutation $\left\{i^{\prime}, j, j^{\prime}\right\} \rightarrow$ $\left\{j, j^{\prime}, i^{\prime}\right\}$.

The Jacobian $|\partial \boldsymbol{C} / \partial \boldsymbol{A}|=|\operatorname{det}(J)|$ is one possible measure of the efficiency of the spin information transfer from $A$ to $C$. If it vanishes, $A$ can vary along in some directions without changing $C$ : this is a loss of information. If $J=0$, the cross section vanishes and obviously no information at all can be transmitted. If $J$ is a pure rotation, $\operatorname{det}(J)=+1$. If it is a rotation times a spin reversal, $\operatorname{det}(J)=-1$ (however this is forbidden, as shown in 2.5.3).

In the case $n_{A}=n_{C}=2$, for instance in $\overrightarrow{\mathrm{p}}+\mathrm{p} \rightarrow \vec{\Lambda}+X$, one can also use the Cartesian components $X^{\mu}$ and $Y^{\mu}$ of $\mathcal{A}$ and $\mathcal{C}$, defined by $\mathcal{A} \equiv \rho_{A}=\frac{1}{2} X^{\mu} \sigma_{A}^{\mu}, \mathcal{C} \equiv Y^{0} \rho_{C}=\frac{1}{2} Y^{\mu} \sigma_{B}^{\mu} . X$ and $\boldsymbol{Y} / Y^{0}$ are the polarisation 3-vectors of $A$ and $C$. The column vector $\boldsymbol{A}$ introduced in (5.19) is related to $X^{\mu}$ by $\boldsymbol{A}=\frac{1}{2}\left(X^{0}+X^{3}, X^{1}-i X^{2}, X^{1}+i X^{2}, X^{0}-X^{3}\right)^{t}$, and similarly for $Y^{\mu}$ and $\boldsymbol{C}$. A third form of (5.20) is

$$
Y^{\nu}=(\operatorname{Tr} \mathcal{R} / 2) X^{\mu} D_{\mu \nu}, \quad \text { with } \quad \operatorname{det}\left(D_{\mu \nu}\right)=(\operatorname{Tr} R / 2)^{-4} \operatorname{det} J .
$$

$D_{\mu \nu}=(\mu 0 \mid \nu 0)$ generalises the "depolarisation parameters" of Sec. 4.1.2. An interesting relation,

$$
\operatorname{det}(\mathcal{R})-\operatorname{det}(J)-\operatorname{det}(\widetilde{\mathcal{R}})=0
$$

links three different information parameters of the reaction:

- $\operatorname{det}(\mathcal{R})$, which has to be positive, is a measure of the "entropy of inclusiveness",

- $\operatorname{det}(J)$ is an algebraic measure of the spin transfer efficiency,

- $-\operatorname{det}(\widetilde{\mathcal{R}})$ is a measure of the "entanglement in the $A \bar{C}$ channel".

For instance $\operatorname{det}(\widetilde{\mathcal{R}})<0$ implies that $\mathcal{R}$ is entangled and that $\operatorname{det}(J)>0$. A simple example is the scattering by a spin-independent potential: $\langle j|\mathcal{M}| i\rangle=a \delta_{i j}$. Then

$$
\frac{\mathcal{R}}{|a|^{2}}=\left(\begin{array}{cccc}
1 & 0 & 0 & 1 \\
0 & 0 & 0 & 0 \\
0 & 0 & 0 & 0 \\
1 & 0 & 0 & 1
\end{array}\right), \quad \frac{J}{|a|^{2}}=\mathbb{1}_{4}=D, \quad \frac{\widetilde{\mathcal{R}}}{|a|^{2}}=\frac{\mathbb{1}+\boldsymbol{\sigma}_{A} \cdot \boldsymbol{\sigma}_{B}}{2}=\left(\begin{array}{cccc}
1 & 0 & 0 & 0 \\
0 & 0 & 1 & 0 \\
0 & 1 & 0 & 0 \\
0 & 0 & 0 & 1
\end{array}\right),
$$

which gives $\operatorname{det}(\mathcal{R})=0, \operatorname{det}(J)=|a|^{8}, \operatorname{det}(\widetilde{\mathcal{R}})=-|a|^{8}$. The negative value of $\operatorname{det}(\widetilde{\mathcal{R}})$ means that $\mathcal{R}$ is entangled. In fact $\langle j|\mathcal{M}| i\rangle \propto \delta_{i j}$ corresponds, in the $t$-channel, to the exchange of the spinsinglet, which is an entangled state. A relation similar to (5.22) can be written for the correlations between two initial or two final spin one-half:

$$
\operatorname{det} \rho_{A B}+\left[\operatorname{Tr}\left(\rho_{A B}\right) / 2\right]^{4} \operatorname{det}\left(C_{\mu \nu}\right)-\operatorname{det} \rho_{A B}^{\mathrm{pt}}=0 .
$$


Inequalities on spin observables

It would be interesting to find an analogous relation for higher spins. These equations, indeed, suggest some general relation between the entanglement of $\mathcal{R}$, the entropy of inclusiveness and the strength of the correlation between $A$ and $B$ or $C$.

\subsection{Domains of quantum positivity, classical positivity and separability}

As we have seen in Sec. 2, the cross section (2.62) of the reaction (2.59) has to be positive for arbitrary independent $\boldsymbol{S}_{A}, \boldsymbol{S}_{B}, \boldsymbol{S}_{C}$ and $\boldsymbol{S}_{D}$ in the unit ball $\boldsymbol{S}^{2} \leq 1$, but this classical condition is not a sufficient condition for the positivity of the cross section matrix. On can thus distinguish a classical positivity domain $\mathcal{D}^{\mathrm{cl}}$, which is larger than the true or quantum positivity domain $\mathcal{D}$. To illustrate this fact, let us consider the final density matrix $\rho_{C+D}$ of a reaction $A+B \rightarrow C+D+X_{f}$. Classical positivity reads $\operatorname{Tr}\left\{\rho_{C+D}\left(\check{\rho}_{C} \otimes \check{\rho}_{D}\right)\right\} \geq 0$ for any acceptance matrices $\check{\rho}_{C}$ and $\check{\rho}_{D}$ of the detectors. More generally

$$
\operatorname{Tr}\left\{\rho_{C+D} \check{\rho}_{C+D}\right\} \geq 0 \text { for any separable } \check{\rho}_{C+D},
$$

whereas quantum positivity requires

$$
\operatorname{Tr}\left\{\rho_{C+D} \check{\rho}_{C+D}\right\} \geq 0 \text { for any separable or entangled } \check{\rho}_{C+D} .
$$

One can say that the classical positivity domain $\mathcal{D}^{\text {cl }}$ of $\rho_{C+D}$ is dual to the separability domain $\mathcal{S}$ of $\check{\rho}_{C+D}$, in the sense that $\operatorname{Tr}\{\rho \eta\} \geq 0$ for any pair $\left\{\rho \in \mathcal{D}^{\mathrm{cl}}, \eta \in \mathcal{S}\right\}$. We recall that the quantum positivity domain $\mathcal{D}$ is dual to itself, according to (2.21). We have

$$
\mathcal{S} \subseteq \mathcal{D} \subseteq \mathcal{D}^{\mathrm{cl}}, \quad \mathcal{S} \subseteq \mathcal{D}^{\mathrm{pt}} \subseteq \mathcal{D}^{\mathrm{cl}},
$$

where $\mathcal{D}^{\mathrm{pt}}$ contains the matrices $\rho$ whose partial transform $\rho^{\mathrm{pt}}$ are semi-positive. Separability and classical positivity are preserved under partial transposition: $\mathcal{S}^{\mathrm{pt}}=\mathcal{S},\left(\mathcal{D}^{\mathrm{cl}}\right)^{\mathrm{pt}}=\mathcal{D}^{\mathrm{cl}}$. All the domains in (5.27) are convex. Figure 38 schematises their respective extensions.

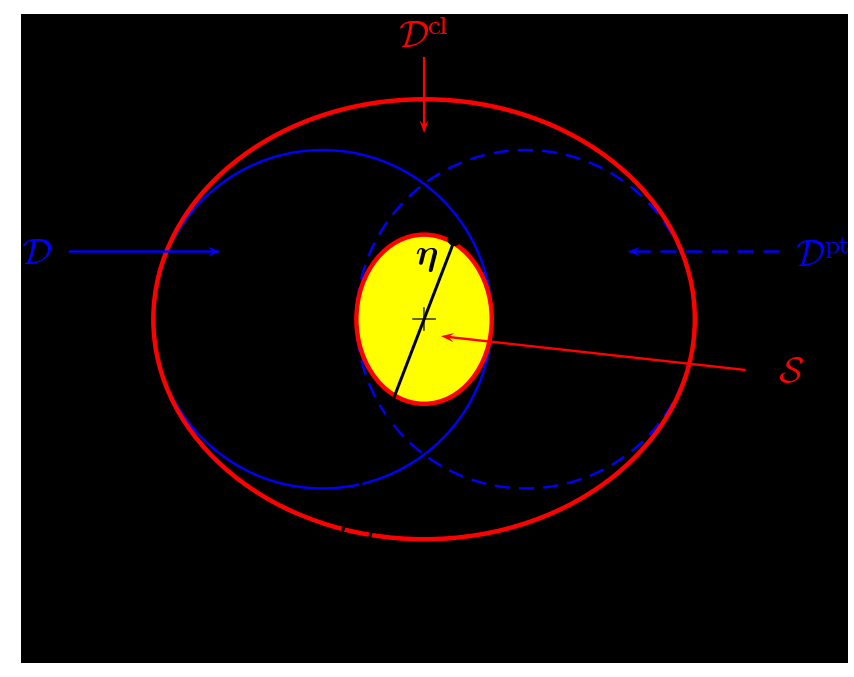

Figure 38: Relative positions of the classical positivity domain $\mathcal{D}^{\text {cl }}$ : the separability domain $\mathcal{S}$, the true positivity domain $\mathcal{D}$ and the domain of positive partial transform $\mathcal{D}^{\mathrm{pt}}$ (dashed contour). $\mathcal{D} \cap \mathcal{D}^{\mathrm{pt}}$ is generally larger than $\mathcal{S}$, but for two spin one-half, one has $\mathcal{D} \cap \mathcal{D}^{\mathrm{pt}}=\mathcal{S}$. A matrix $\eta$ of the boundary $\partial \mathcal{S}$ is represented together with its reciprocal polar line, which is tangent to $\partial \mathcal{D}^{\mathrm{cl}}$.

In analogy with (2.21), we can write the duality between $\mathcal{D}^{\mathrm{cl}}$ and $\mathcal{S}$ as

$$
\boldsymbol{\rho}_{\perp} \cdot \boldsymbol{\eta}_{\perp} \geq-1 / N \text { for all } \rho \in \mathcal{D}^{\mathrm{cl}}, \eta \in \mathcal{S},
$$


after normalisation of $\rho$ and $\eta$ to unit trace. This implies that the boundaries $\partial \mathcal{D}^{\mathrm{cl}}$ and $\partial \mathcal{S}$ of the two domains are polar reciprocal of each other, as shown in Fig. 38. This reciprocity is also visible on a subset of observables, in the following form: let $P$ be the hyperplane defined by the vanishing of all other observables, $P\left(\mathcal{D}^{\mathrm{cl}}\right)$ and $P(\mathcal{S})$ denote the projections of $\mathcal{D}^{\mathrm{cl}}$ and $\mathcal{S}$ on $P$. In analogy with (2.23) we have

$$
\boldsymbol{\rho}_{\perp} \cdot \boldsymbol{\eta}_{\perp} \geq-1 / N \quad \text { for all } \quad \rho \in P \cap \mathcal{D}^{\mathrm{cl}}, \eta \in P(\mathcal{S}) \quad \text { or } \quad \eta \in P\left(\mathcal{D}^{\mathrm{cl}}\right), \eta \in P \cap \mathcal{S} .
$$

For instance, for a two-fermion system of density matrix $\rho_{C+D}=\frac{1}{4} C_{\mu \nu} \sigma_{\mu}(C) \otimes \sigma_{\nu}(D)$, the classical positivity domain of the triple $\left\{C_{x x}, C_{y y}, C_{z z}\right\}$ is the whole cube $[-1,+1]^{2}$, the quantum positivity domain is the tetrahedron defined by

$$
C_{x x}-C_{y y}-C_{z z} \leq 1 \text { and circular permutations, } C_{x x}+C_{y y}+C_{z z} \leq 1,
$$

and the separability domain, an octahedron, is the intersection of the tetrahedron with its mirror figure. One can see on Fig. 39 the polar reciprocity (edge $\leftrightarrow$ edge) and (summit $\leftrightarrow$ face) between the cube and the octahedron. Here the full domains $\mathcal{S}, \mathcal{D}$ and $\mathcal{D}^{\text {cl }}$ are symmetrical about $P$, therefore their intersections with $P$ coincide with their projections on $P$.

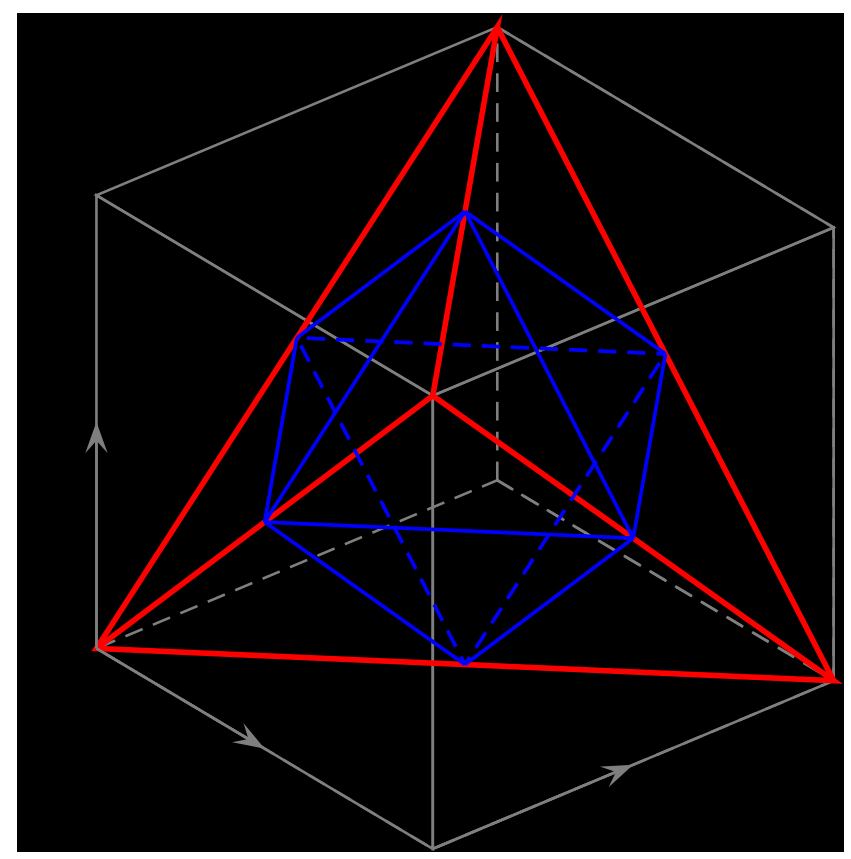

Figure 39: Classical positivity domain (cube), true positivity domain (tetrahedron) and separability domain (octahedron) for the triple $\left\{C_{x x}, C_{y y}, C_{z z}\right\}$ of observables. 


\section{Conclusions and outlook}

Spin dynamics and inequalities In this review, we have discussed several aspects of spin physics, as studied in exclusive hadronic reactions, lepton-induced reactions, inclusive reactions and in particular spin-dependent structure functions and analogous aspects in generalised parton distributions and BFKL evolution.

The main focus of this survey is on the inequalities relating spin observables. When suitably projected, these inequalities delimit the domain allowed for two, three or more observables. In the case of a pair, the domain is a disk or a triangle inside the unit square, but their are many cases where the entire square is accessible. For a triple of observables of a reaction involving less than eight amplitudes, there is always a constraint, and the geometry of the allowed domain can assume a variety of shapes: sphere, cone, pyramid, tetrahedron, octahedron, intersection of cylinders, etc.

To list the inequalities, different methods can be used. For a preliminary investigation, it is possible to generate randomly fictitious amplitudes and to plot an observable against the other ones. Once the domain is identified, its boundary can be derived algebraically from the explicit expression of the observables in terms of a set of independent amplitudes, an exercise that is not always easy. The positivity of the density matrix of the initial or final state, or of a state relevant for a crossed reaction, gives some physics insight into the origin of these inequalities.

The inequalities reviewed in this article are not relevant for the cases where only one spin observable is measured, for instance one polarisation or one analysing power. They are also not needed in the rare cases (for instance elastic nucleon-nucleon scattering at low energy), where a reliable amplitude analysis has been carried out which fits well the data points: the inequalities are then automatically satisfied. The optimal circumstances for using these inequalities are those where a few observables are measured, and some further ones are foreseen: it is possible to check the compatibility of the existing data, the range allowed for the future ones, although a complete amplitude analysis is yet out of reach.

Examples have been chosen to illustrate the formalism. For exclusive reactions, the cases of $\pi \mathrm{N} \rightarrow \pi \mathrm{N}, \overline{\mathrm{p}} \mathrm{p} \rightarrow \pi \pi, \overline{\mathrm{p}} \mathrm{p} \rightarrow \bar{\Lambda} \Lambda, \gamma \mathrm{p} \rightarrow \mathrm{K} \Lambda$ and $\gamma \mathrm{p} \rightarrow \rho \mathrm{N}$ have been described in details. The domains for the spin observables are of course identical for reactions with exactly the same spin structure, such as $\mathrm{KN} \rightarrow \mathrm{KN}$, whose detailed investigation has been proposed in relation with the tentative pentaquark, and $\pi \mathrm{N} \rightarrow \pi \mathrm{N}$. For $\overline{\mathrm{p}} \mathrm{p} \rightarrow \mathrm{e}^{+} \mathrm{e}^{-}$, as compared to $\overline{\mathrm{p}} \mathrm{p} \rightarrow \bar{\Lambda} \Lambda$, further restrictions applied if the leading mechanism is one-photon exchange in the $s$-channel. Some reactions are related by crossing, and hence are constrained by similar inequalities, once crossing is applied to the observables. This is the case, for instance, for the photoproduction of vector mesons and the photodisintegration of the deuteron.

For inclusive reactions, the constraint on the spin-dependent integrated cross-section has been reminded, i.e., the inequality relating $\sigma_{\text {tot }}, \Delta \sigma_{T}$ and $\Delta \sigma_{L}$. For reactions $a+b \rightarrow c+X$, with $a, b$ or $c$ polarised or analysed, there are inequalities for the spin-correlation or spin-transfer observables. Inequalities are also for the structure functions and parton distributions. A king of universality is encountered, with the same shape observed for different exclusive reactions and for the inclusive reactions which have been studied, for instance the triangle $2|y| \leq 1+x$ and the tetrahedron $x \pm y \leq$ $1 \mp z$ (or its mirror image).

On the theoretical side, a number of questions have been discussed along this review and clarified, although, perhaps, alternative explanations could be envisaged. For instance, what is the maximal degree of the limiting curves and surfaces? In our investigations, this degree is always either 1 or 2 . Why are the allowed domain convex? What is the minimal number $n$ of amplitudes needed to envisage that an hypercube $[-1,+1]^{m}$ becomes fully allowed for $m$ observables? How to distinguish genuinely quantum effects from inequalities which can be understood at the classical level?

New polarised beams In the near future, new experiments are expected to take data, either at existing accelerators with improved polarisation devices, or at new facilities designed with polarisation equipment. Much progress is observed in the art of building and maintaining a high degree of 
polarisation in targets, see, e.g., [271] for the history and [272] for the recent developments.

Polarised beams of electrons, protons, neutrons or photons are routinely produced, and a hot issue at the time of completion of this review is whether intensive polarised beams of positrons or antiprotons can be built. The physics case of polarised positrons at the future ILC (International Linear Collider) is discussed, e.g., in [273]. Several devices have been proposed, for instance, the production of high-energy polarised photons by back scattering of an intense electron beam on a polarised laser and pair creation by these photons [274].

The problem of polarising antiprotons was debated in the 80's when LEAR came into operation at CERN, but was not given very high priority in the CERN agenda. Debates were also organized in view of implementing polarisation at the Fermilab collider [275]. The discussion is now resumed for studying antiproton-induced reactions at FAIR or JParc.

As polarised protons have been produced and used in the E704 experiment at Fermilab from the weak decay $\Lambda \rightarrow \mathrm{p} \pi$, polarised antiprotons have be obtained in a similar way [212,276]. This method could be further used, provided a large enough number $\bar{\Lambda}$ can be produced. See, also, [275], and Refs. there.

Another idea is to polarise an antiproton by nuclear scattering on nuclei, a method known to work in the case of protons. Antiprotons appear, however, as weakly polarised when scattered off carbon $[25,277]$. Other nuclei might be more favourable. It is even better to use inelastic scattering of antiprotons, rather than elastic. The reaction is $\bar{p}+A \rightarrow \bar{p}+A^{*}$, where $A$ is the nucleus ground-state and $\mathrm{A}^{*}$ one of its excited states, with well-chosen quantum numbers. As stressed years ago by Dover [278-280], and more recently by Yu-shun et al. [281], such inelastic transition "filters" specific spin-isospin components of the antiproton-nucleon interaction. If, e.g., the role of the tensor force is enhanced, a good transfer of polarisation from a longitudinally polarised nuclear target target to the antiproton might be expected. A spin-orbit term in the effective antiproton-nucleus potential (which might result from the tensor component of the antinucleon-nucleon potential) should also produce a good transverse polarisation of the scattered antiproton [281]. This would deserve further studies.

A "spin-splitter" has been studied [282-284]. It is basically a Stern-Gerlach device, where an inhomogeneous magnetic field pushes up the spin states $\sigma_{z}=+1$ and down those with $\sigma_{z}=$ -1 . A test was even conducted with protons the IUCF [285], which cannot be considered as fully conclusive.

The "spin-filter" has also been much discussed: unpolarised antiprotons are maintained circulating in a ring where they regularly hit a polarised proton target. Then, if $\Delta \sigma_{\mathrm{T}}=\sigma(\uparrow \downarrow)-\sigma(\uparrow \uparrow)$ is large enough (as compared to $\sigma_{\text {tot }}$, the beam of remaining antiprotons become more and more polarised. Tests have been made with protons in a dedicated ring at Heidelberg. However, the tests were not conclusive enough at the time when LEAR was shut down. Also, this method might work better with longitudinal polarisation, as $\left|\Delta \sigma_{L}\right|>\left|\Delta \sigma_{T}\right|$ at least in some model calculations, but it is easier to keep transverse than longitudinal polarisation in a ring.

A potential improvement is to use the polarisation of the electron of the hydrogen target, or even better to combine optimally the electron and proton contributions by comparing the results from polarised ortho- or para-hydrogen.

A recent variant relies solely on the (known) electron-antiproton interaction and uses a spin transfer from an electron-polarised hydrogen gas target to an antiproton beam. The estimate for the antiproton beam foreseen at the future GSI facility is a polarisation of the order of 0.2-0.4 [286]. However, another study does not confirm this optimistic result [287].

Studies have even be done of arranging a circulation of the antiproton beam within a parallel beam of polarised electrons, but so far the results are not fully convincing.

Note however, that if antiprotons have so far resisted attempts to make them polarised, it could be easier to produce polarised antineutrons. By the the charge-exchange reaction $\bar{p} p \rightarrow \bar{n} n$, antineutron beams have been produced at CERN and Brookhaven and used to study antineutron induced reactions. As a large transfer of polarisation is expected, an antineutron beam with high longitudinal polarisation can result if the charge exchange reaction is performed on a longitudinally polarised 
hydrogen target [288-290].

\section{Acknowledgements}

We benefited from many discussions with several colleagues on various aspects of spin physics. We thank M. Asghar for useful comments on the manuscript. The hospitality extended to us for a collaboration meeting at ECT*, Trento, is gratefully acknowledged. 


\section{References}

[1] C. Bourrely, J. Soffer and E. Leader, "Polarisation phenomena in hadronic reactions", Phys. Rept. 59 (1980) 95.

[2] E. Leader, "Spin in particle physics", Camb. Monogr. Part. Phys. Nucl. Phys. Cosmol. 15 (2001) 1 .

[3] A. Pais, "Inward bound: of matter and forces in the physical world", Oxford, UK: Clarendon. New York, USA: Oxford Univ. Pr. ( 1986) 666p.

[4] L.M. Brown and H. Rechenberg, "The origin of the concept of nuclear forces", Bristol, UK: IOP (1996) $392 \mathrm{p}$.

[5] J. Bystricky, F. Lehar and P. Winternitz, "Formalism of nucleon-nucleon elastic scattering experiments", J. Phys. (France) 39 (1978) 1.

[6] C. Lechanoine-Leluc and F. Lehar, "Nucleon-nucleon elastic scattering and total crosssections", Rev. Mod. Phys. 65 (1993) 47.

[7] M. Sarsour et al., "Measurement of the Absolute np Scattering Differential Cross Section at 194 MeV”, Phys. Rev. Lett. 94 (2005) 082303.

[8] B. von Przewoski et al., "Analyzing Powers and Spin Correlation Coefficients for $\mathrm{p}+\mathrm{d}$ Elastic Scattering at 135 and $200 \mathrm{MeV}$ ", Phys. Rev. C74 (2006) 064003.

[9] H.O. Meyer, "Polarization experiments with storage rings", Phys. Scripta T104 (2003) 19.

[10] F. Bauer et al., "Measurement of spin correlation parameters $A_{N N}, A_{S S}$, and $A_{S L}$ at $2.1 \mathrm{GeV}$ in proton-proton elastic scattering", Phys. Rev. Lett. 90 (2003) 142301.

[11] EDDA, F. Hinterberger and K.O. Eyser, "Elastic proton-proton scattering at GeV energies: Analyzing powers $A_{N}$ and spin correlation parameters $A_{N N}, A_{S S}$, and $A_{S L}$ ", Int. J. Mod. Phys. A20 (2005) 1959.

[12] M. Altmeier et al., "Excitation functions of the analyzing power in elastic proton proton scattering from $0.45 \mathrm{GeV}$ to $2.5 \mathrm{GeV}$ ", Eur. Phys. J. A23 (2005) 351.

[13] F. Lehar, "Current experiments using polarized beams of the JINR LHE accelerator complex", Phys. Part. Nucl. 36 (2005) 501.

[14] M. Lacombe et al., "Parametrization of the Paris NN potential”, Phys. Rev. C21 (1980) 861.

[15] R. Machleidt, "The high-precision, charge-dependent Bonn nucleon-nucleon potential (CDBonn)", Phys. Rev. C63 (2001) 024001.

[16] E. Epelbaum, W. Glöckle and U.G. Meissner, "The two-nucleon system at next-to-next-tonext-to-leading order”, Nucl. Phys. A747 (2005) 362.

[17] R. De Vita, "Soft spin physics at Jefferson Laboratory", Prepared for 16th International Spin Physics Symposium (SPIN 2004), Trieste, Italy, 10-16 Oct 2004.

[18] K. de Jager, "Future spin physics at JLab: 12-GeV and beyond", (2006), nucl-ex/0612025, 17th International Spin Physics Symposium (SPIN06), Kyoto, Japan, 2-7 Oct 2006.

[19] M. Ripani, "Nucleon Spin Physics with CLAS at JLab", Nucl. Phys. A782 (2007) 110, International Conference on Particle-Nucleus and Nucleus-Nucleus Scattering at Relativistic Energies, Trieste, Italy, 22-26 May 2006. 
Inequalities on spin observables

[20] W.M. Kloet, W.T. Chiang and F. Tabakin, "Spin information from vector-meson decay in photoproduction", Phys. Rev. C58 (1998) 1086.

[21] W.M. Kloet and F. Tabakin, "Constraints on vector meson photoproduction spin observables", Phys. Rev. C61 (2000) 015501.

[22] W.M. Kloet and F. Tabakin, "Vector meson photoproduction - Model independent aspects", Nucl. Phys. A675 (2000) 325c.

[23] L. Durand III and J. Sandweiss, "Spin Correlation Phenomena in the Reaction $\overline{\mathrm{N}}_{a}+\mathrm{N}_{b} \rightarrow$ $\overline{\mathrm{Y}}_{c}+\mathrm{Y}_{d}$ ”, Physical Review 135 (1964) B540.

[24] G. Cohen-Tannoudji and A. Messiah, "A measure of the polarization of high-energy $\bar{p}$ beam from angular correlation in reaction $\overline{\mathrm{p}} \mathrm{p} \rightarrow \overline{\mathrm{Y} Y}$ ", Nuovo Cim. 33 (1964) 853.

[25] E. Klempt et al., "Antinucleon nucleon interaction at low energy: Scattering and protonium", Phys. Rept. 368 (2002) 119.

[26] M. Alberg, J.R. Ellis and D. Kharzeev, "The proton spin puzzle and depolarization in $\bar{p} p \rightarrow$ $\bar{\Lambda} \Lambda ”$, Phys. Lett. B356 (1995) 113.

[27] J.-M. Richard, "Remarks about spin measurements in $\bar{p} p \rightarrow \bar{\Lambda} \Lambda$ ", Phys. Lett. B369 (1996) 358.

[28] M. Elchikh and J.-M. Richard, "Constraints on spin observables in $\bar{p} p \rightarrow \bar{\Lambda} \Lambda$ ", Phys. Rev. C61 (2000) 035205.

[29] J.-M. Richard and X. Artru, "Constraints on spin observables in $\bar{p} p \rightarrow \bar{\Lambda} \Lambda$ ", Nucl. Instrum. Meth. B214 (2004) 171, Proc. LEAP03, Yokohama, Japan, Jan. 2003.

[30] M. Elchikh, "Density matrix constraints on spin observables in $\overline{\mathrm{p}}+\mathrm{p} \rightarrow \bar{\Lambda}+\Lambda$ ", Acta Phys. Polon. B35 (2004) 2439.

[31] X. Artru and J.-M. Richard, "General constraints on spin observables: Applications to $\overline{\mathrm{p}}+\mathrm{p} \rightarrow \bar{\Lambda}+\Lambda$ and to polarized quark distributions", Phys. Part. Nucl. 35 (2004) S126, Proc. Dubna Spin Workshop.

[32] G. Bunce et al., "Prospects for spin physics at RHIC", Ann. Rev. Nucl. Part. Sci. 50 (2000) 525 .

[33] S.J. Brodsky, D.S. Hwang and I. Schmidt, "Final-state interactions and single-spin asymmetries in semi-inclusive deep inelastic scattering", Phys. Lett. B530 (2002) 99.

[34] D. Boer, "Investigating the origins of transverse spin asymmetries at RHIC", Phys. Rev. D60 (1999) 014012.

[35] D. Boer, S.J. Brodsky and D.S. Hwang, "Initial state interactions in the unpolarized Drell-Yan process", Phys. Rev. D67 (2003) 054003.

[36] T.D. Lee and C.N. Yang, "Possible determination of the spin of $\Lambda^{0}$ from its large decay angular asymmetry", Phys. Rev. 109 (1958) 1755.

[37] R.H. Dalitz, "Properties of the weak interactions", Lectures given at Int. School of Physics Enrico Fermi, Varenna, Italy, Jun 1964.

[38] M. Ademollo, R. Gatto and G. Preparata, "Density-Matrix Analysis for Spin Determination", Phys. Rev. 140 (1965) B192. 
[39] M.G. Doncel, L. Michel and P. Minnaert, Matrices densité de polarisation, Lectures at the Summer School of Gif-sur-Yvette, 1970, Ed. R. Salmeron, Laboratoire de Physique, Ecole Polytechnique, Paris (1970).

[40] P. Minnaert, The polarization domain, in Les Houches Summer School on Theoretical Physics, Session 21, 1971, Particle Physics, Eds. C. de Witt and C. Itzykson, Gordon and Breach, New York, 1973, p.115.

[41] P. Minnaert, "Positivity Conditions for the Density Matrix of Spin-One Particles", Phys. Rev. Lett. 16 (1966) 672, (E), ibid. 1030.

[42] P. Minnaert, "Spin-Density-Matrix Analysis. Positivity Conditions and Eberhard-Good Theorem", Phys. Rev. 151 (1966) 1306.

[43] J. Daboul, Conditions on density matrix elements and their application in resonance production, Nucl. Phys. B 4 (1967) 180.

[44] R.M. Delaney and J.L. Gammel, "Quadratic relations among observables in pp scattering", Phys. Rev. D12 (1975) 1978.

[45] C. Bourrely and J. Soffer, "Sixteen relations between observables in nucleon-nucleon scattering", Phys. Rev. D12 (1975) 2932.

[46] N. P. Klepikov, Z.Sh. Kogan, S.V. Shamanin, Identities between observables in the scattering of particle with spin, Sov. J. Nucl. Phys. 5 (1967) 928.

[47] P. La France and P. Winternitz, "Nonlinear relations between observables in the scattering of spinor particles and the direct reconstruction of the scattering matrix", Phys. Rev. D27 (1983) 112.

[48] V. Barone, A. Drago and P.G. Ratcliffe, "Transverse polarisation of quarks in hadrons", Phys. Rept. 359 (2002) 1.

[49] G. Ohlsen, "Polarization transfer and spin correlation experiments in nuclear physics", Rep. Prog. Phys. 35 (1972) 717.

[50] J. Ashkin et al., "Convention for Spin Parameters in High-Energy Scattering Experiments", AIP Conf. Proc. 42 (1978) 142.

[51] V.B. Berestetskii, E.M. Lifshitz and L.P. Pitaevskii, Quantum electrodynamics (Butterworth (Oxford, UK), 1982).

[52] K. Schilling, P. Seyboth and G.E. Wolf, "On the analysis of vector meson production by polarized photons", Nucl. Phys. B15 (1970) 397.

[53] A.V. Efremov, L. Mankiewicz and N.A. Törnqvist, "On the handedness in polarized $\tau \rightarrow$ $\mathrm{a}_{1} \nu \rightarrow 3 \pi \nu$ decay", Phys. Lett. B291 (1992) 473.

[54] M. Jacob and G.C. Wick, "On the general theory of collisions for particles with spin", Ann. Phys. 7 (1959) 404.

[55] J. S. Bell, “On the Einstein-Podolsky-Rosen paradox,” Physics 1 (1964) 195.

[56] A. Peres, "Separability Criterion for Density Matrices”, Phys. Rev. Lett. 77 (1996) 1413.

[57] M. Horodecki, P. Horodecki and R. Horodecki, "On the necessary and sufficient conditions for separability of quantum mixed states”, Phys. Lett. A223 (1996) 1. 
Inequalities on spin observables

[58] M. Horodecki, P. Horodecki and R. Horodecki, "Separability of $n$-particle mixed states: necessary and sufficient conditions in terms of linear maps", Phys. Lett. A283 (2001) 1.

[59] L.D. Landau et al., Course Theor. Phys., Relativistic Quantum Theory, Part. II (Pergamon, Oxford, 1974).

[60] X. Artru, "Classical and quantum constraints in spin physics", Advanced Research Workshop on High Energy Spin Physics (ed. A.V. Efremov and S.V. Goloskokov), Dubna (2008) p. 26.

[61] X. Artru, J.-M. Richard and J. Soffer, "Positivity domains for pairs of triples of spin observables", Advanced Research Workshop on High Energy Spin Physics (ed. A.V. Efremov and S.V. Goloskokov), Dubna (2008) p. 21.

[62] Particle Data Group, W.M. Yao et al., "Review of particle physics”, J. Phys. G33 (2006) 1.

[63] A. Bohr, "Relation between intrinsic parities and polarizations in collision and decay processes", Nucl. Phys. 10 (1959) 486.

[64] W. Roberts and T. Oed, "Polarization observables for two-pion production off the nucleon", Phys. Rev. C71 (2005) 055201.

[65] S.J. Brodsky, C.E. Carlson and H.J. Lipkin, "Spin Effects in Large Transverse Momentum Exclusive Scattering Processes", Phys. Rev. D20 (1979) 2278.

[66] C.K. Chen, "Pure Vector Type Quark Quark Interaction and the Spin Dependence of Large Angle Elastic Nucleon-Nucleon Scattering", Phys. Rev. Lett. 41 (1978) 1440.

[67] G.R. Farrar et al., "Constituent Description of n n Elastic Scattering Observables at Large Angles”, Phys. Rev. D20 (1979) 202.

[68] D.W. Sivers, "Single spin production asymmetries from the hard scattering of point-like constituents", Phys. Rev. D41 (1990) 83.

[69] D. Boer and P.J. Mulders, "Time-reversal odd distribution functions in leptoproduction", Phys. Rev. D57 (1998) 5780.

[70] X. Artru and M. Mekhfi, "Transversely polarized parton densities, their evolution and their measurement", Z. Phys. C45 (1990) 669.

[71] X. Artru and M. Mekhfi, "What can we learn from unpolarized and polarized electroproduction of fast baryons?”, Nucl. Phys. A532 (1991) 351.

[72] X. Artru, "The transverse spin", (2002), in Proc. $10^{\text {th }}$ Rhodanian Seminar: The Spin in Physics, Turin, Italy, March 2002, ed. M. Anselmino et al., Frontier Group, Virginal-Ittre, Belgium (2002), hep-ph/0207309.

[73] K.I. Hikasa, "Transverse polarization effects in $e^{+} e^{-}$collisions: the role of chiral symmetry", Phys. Rev. D33 (1986) 3203.

[74] C. Bourrely, L. Michel and J. Soffer, in Proceedings Workshop "Polarized proton ion sources' (Ann Arbor, Michigan, 1980) edited by A.D. Kirsch and A.T.M. Lin. N.Y., American Inst. Phys, 1982. 214p. (AIP Conference Proceedings, 80); see also [1], p. 291.

[75] M. Korkea-Aho, N.A. Törnqvist,, Lett. Nuovo Cim. 2 (1971) 1305.

[76] M.G. Doncel, L. Michel and P. Minnaert, "Constraints on spin rotation parameters due to isospin conservation", Phys. Lett. B38 (1972) 42. 
[77] M.G. Doncel, L. Michel and P. Minnaert, "Isospin constraints between three cross-sections and two polarization density matrices", Phys. Lett. B42 (1972) 96.

[78] N.A. Törnqvist, "New phenomena in $\pi+\mathrm{N} \rightarrow \pi+\mathrm{N}$ : saturation of the most stringent isospin bound", Phys. Lett. B47 (1973) 449.

[79] M. Jacob and G.F. Chew, Strong Interaction Physics (Benjamin, 1964).

[80] R.A. Arndt et al., "Pion-nucleon partial wave analysis to 2 GeV", Phys. Rev. D43 (1991) 2131.

[81] V.V. Abaev et al., "Measurement of the spin rotation parameters $R$ and $A$ in $\pi-p$ elastic scattering in the region of pion nucleon resonances", in Proc. $8^{\text {th }}$ Int. Symp. High-Energy Spin Physics, Minneapolis (MN), 12-17 Sep 1988, AIP Conf. Proc. 187, Vol. 1, p. 676-680 (AIP, N.Y., 1989).

[82] I. Supek et al., "Spin rotation parameters $A$ and $R$ for $\pi^{+} \mathrm{p}$ and $\pi^{-} \mathrm{p}$ elastic scattering from $427 \mathrm{MeV} / c$ to $657 \mathrm{MeV} / c$ ", Phys. Rev. D47 (1993) 1762.

[83] J. Pierrard et al., "On measuring spin rotation parameter $R$ in $\pi \mathrm{p}$ and $\mathrm{K}^{-} \mathrm{p}$ elastic scattering at $40 \mathrm{GeV} / c$ ", Phys. Lett. B57 (1975) 393.

[84] A. Gaidot et al., "Polarization measurements in $\pi^{-} \mathrm{p}, \mathrm{K}^{-} \mathrm{p}$ and $\mathrm{p} \mathrm{p}$ elastic scattering at 40 GeV/c", Phys. Lett. B57 (1975) 389.

[85] W.R. Frazer and J.R. Fulco, "Partial-wave dispersion relations for the process $\pi+\pi \rightarrow \mathrm{N}+\overline{\mathrm{N}}$ ", Phys. Rev. 117 (1960) 1603.

[86] A. Hasan et al., "Differential cross-sections and analyzing powers for $\bar{p} p \rightarrow \pi^{-} \pi^{+}$and $\mathrm{K}^{-} \mathrm{K}^{+}$from $360-\mathrm{MeV} / c$ to $1550-\mathrm{MeV} / c^{\prime \prime}$, Nucl. Phys. B378 (1992) 3.

[87] M.N. Oakden and M.R. Pennington, "Amplitude analysis of data on $\bar{p} p \rightarrow \pi \pi$ at lowenergy", Nucl. Phys. A574 (1994) 731.

[88] S. Takeuchi, F. Myhrer and K. Kubodera, "Maximum asymmetry phenomena in $\overline{\mathrm{p}} \mathrm{p} \rightarrow \pi^{-} \pi^{+}$ and $\overline{\mathrm{p}} \mathrm{p} \rightarrow \mathrm{K}^{-} \mathrm{K}^{+}$reactions", Nucl. Phys. A556 (1993) 601.

[89] M. Elchikh and J.-M. Richard, "Meson exchange and nonlocality effects in proton antiproton annihilation into two pseudoscalar mesons", Phys. Rev. C48 (1993) 17.

[90] C.B. Dover and J.-M. Richard, "Strong tensor forces and the possibility of high spin states in the nucleon - antinucleon system", Phys. Rev. D17 (1978) 1770.

[91] K. Paschke, Carnegie Mellon University Thesis (2001), available at: http://www.phys.cmu.edu/ppaschke/.

[92] PS185, B. Bassalleck et al., "Measurement of spin transfer observables in $\bar{p} p \rightarrow \bar{\Lambda} \Lambda$ at 1.637 GeV/c", Phys. Rev. Lett. 89 (2002) 212302.

[93] B. Bassalleck et al., "Depolarization and spin transfer in $\bar{p} p \rightarrow \bar{\Lambda} \Lambda$ with a polarized target", Nucl. Instrum. Meth. B214 (2004) 167.

[94] PS185, K.D. Paschke et al., "Experimental determination of the complete spin structure for $\overline{\mathrm{p}} \mathrm{p} \rightarrow \bar{\Lambda} \Lambda$ at $p_{\text {lab }}=1.637 \mathrm{GeV} / c$ ", Phys. Rev. C74 (2006) 015206.

[95] A. Le Yaouanc et al., "Hadron transitions in the quark model", Gordon and Breach, NewYork, USA (1988). 
Inequalities on spin observables

[96] J. Haidenbauer et al., "Meson exchange and quark-gluon transitions in the $\bar{p} p \rightarrow \bar{\Lambda} \Lambda$ process", Phys. Rev. C46 (1992) 2158.

[97] M. Elchikh and J.-M. Richard, "Spin observables in $\bar{p} p \rightarrow \bar{\Lambda} \Lambda$ and density-matrix constraints", AIP Conf. Proc. 796 (2005) 108.

[98] B. Holzenkamp, K. Holinde and J. Speth, "A meson exchange model for the hyperon nucleon interaction", Nucl. Phys. A500 (1989) 485.

[99] J. Haidenbauer, K. Holinde and J. Speth, "A Meson exchange model for the antihyperonhyperon production”, Nucl. Phys. A562 (1993) 317.

[100] K. Paschke and B. Quinn, "Spin observables in $\bar{p} p \rightarrow \bar{\Lambda} \Lambda$ with a transverse initial state polarization", Phys. Lett. B495 (2000) 49.

[101] A. Antonelli et al., "Measurement of the electromagnetic form-factor of the proton in the timelike region", Phys. Lett. B334 (1994) 431.

[102] G. Bardin et al., "Precise determination of the electromagnetic form-factor of the proton in the timelike region up to $s=4.2 \mathrm{GeV}^{2}$ ", Phys. Lett. B257 (1991) 514 .

[103] N.H. Buttimore and E. Jennings, "Helicity amplitudes and crossing relations for antiproton proton reactions", Eur. Phys. J. A33 (2007) 21.

[104] S.M. Bilenky, C. Giunti and V. Wataghin, "The Process $\bar{p} p \rightarrow \mathrm{e}^{-} \mathrm{e}^{+}$with polarized initial particles and proton form-factors in timelike region", Z. Phys. C59 (1993) 475.

[105] N.H. Buttimore and E. Jennings, "Polarisation observables in lepton antilepton to proton antiproton reactions including lepton mass", Eur. Phys. J. A31 (2007) 9.

[106] T. Barnes, X. Li and W. Roberts, “Evidence for a J $/ \psi$ - pp̄ Pauli Strong Coupling?”, (2007), arXiv:0709.4491 [hep-ph].

[107] J.R. O’Fallon et al., “Spin-spin interactions in high $p_{T}^{2}$ elastic $p p$ scattering”, Phys. Rev. Lett. 39 (1977) 733.

[108] D.G. Crabb et al., "Spin Dependence of High p-Transverse**2 Elastic p p Scattering", Phys. Rev. Lett. 41 (1978) 1257.

[109] E.A. Crosbie et al., "Energy Dependence of Spin Spin Effects in p p Elastic Scattering at 90-Degrees Center-Of-Mass", Phys. Rev. D23 (1981) 600.

[110] W.R. Gibbs and B. Loiseau, "Two-pion exchange contribution to proton-proton scattering at medium energies", Phys. Rev. C77 (2008) 014003.

[111] Particle Data Group, Durham Reaction Data Base, http://durpdg.dur.ac.uk/.

[112] P.J. Bussey et al., "The polarized beam asymmetry in photoproduction of eta mesons from protons at 2.5 GeV and 3.0 GeV”, Phys. Lett. B61 (1976) 479.

[113] The GRAAL, O. Bartalini et al., "Measurement of eta photoproduction on the proton from threshold to $1500 \mathrm{MeV}$ ", Eur. Phys. J. A33 (2007) 169.

[114] LEPS, N. Muramatsu, "Recent results and future prospects at SPring-8 LEPS experiment", AIP Conf. Proc. 870 (2006) 455, Proc. CIPANP 2006, Puerto Rico, 2006.

[115] F. Klein, "Overview on the physics program at ELSA / Bonn", AIP Conf. Proc. 870 (2006) 449, Talk given at CIPANP 2006, Puerto-Rico, June 2006. 
[116] GRAAL, C. Schaerf, "The GRAAL Collaboration: Results and prospects", Prepared for International Workshop on the Physics of Excited Baryons (NSTAR 05), Tallahassee, Florida, 10-15 Oct 2005.

[117] CLAS, M. Garçon, "Physics with CLAS at Jefferson Lab", Nucl. Phys. A782 (2007) 426.

[118] GRAAL, O. Bartalini et al., "Measurement of $\pi^{0}$ photoproduction on the proton from 550 MeV to $1500 \mathrm{MeV}$ at GRAAL", Eur. Phys. J. A26 (2005) 399.

[119] CLAS, R. Bradford et al., "First measurement of beam-recoil observables $\mathrm{C}(\mathrm{x})$ and $\mathrm{C}(\mathrm{z})$ in hyperon photoproduction", Phys. Rev. C75 (2007) 035205.

[120] G.F. Chew et al., "Relativistic dispersion relation approach to photomeson production", Phys. Rev. 106 (1957) 1345.

[121] I.S. Barker, A. Donnachie and J.K. Storrow, "Complete experiments in pseudoscalar photoproduction", Nucl. Phys. B95 (1975) 347.

[122] R.L. Walker, "Phenomenological analysis of single pion photoproduction", Phys. Rev. 182 (1969) 1729.

[123] G.R. Goldstein et al., "Spin correlation measurements in pseudoscalar meson photoproduction", Nucl. Phys. B 80 (1974) 164;

J. Frøyland, in Proc. 1971 Int. Symp. on Electron and Photon Interactions, ed. N.B. Mistry. Ithaca, N.Y., Lab. of Nuclear Studies, Cornell University, 1972;

J. Frøyland, in Springer Tracts in Modern Physics, vol. 63, ed. G. Höhler, (Springer-verlag, Berlin, 1972);

R.C. Worden, "Regge models of forward pion and eta photoproduction", Nucl. Phys. B37 (1972) 253.

[124] W.T. Chiang and F. Tabakin, "Completeness rules for spin observables in pseudoscalar meson photoproduction”, Phys. Rev. C55 (1997) 2054.

[125] X. Artru, J.-M. Richard and J. Soffer, "Positivity constraints on spin observables in exclusive pseudoscalar meson photoproduction", Phys. Rev. C75 (2007) 024002.

[126] C.G. Fasano, F. Tabakin and B. Saghai, "Spin observables at threshold for meson photoproduction”, Phys. Rev. C46 (1992) 2430.

[127] R. Schumacher, "Polarization of hyperons in elementary photoproduction", (2006), nuclex/0611035.

[128] Simultaneous analysis of recent CLAS and GRAAL data on $\gamma \mathrm{p} \rightarrow \mathrm{K} \Lambda$, in preparation. Private communication.

[129] Jefferson Lab Hall A, X. Jiang et al., "Recoil-Proton Polarization in High-Energy Deuteron Photodisintegration with Circularly Polarized Photons", Phys. Rev. Lett. 98 (2007) 182302.

[130] V. Dmitrasinovic and F. Gross, "Polarization observables in deuteron photodisintegration and electrodisintegration", Phys. Rev. C40 (1989) 2479, Erratum, C43 (1991) 1495.

[131] F.J. Gilman, "Photoproduction and electroproduction”, Phys. Rept. 4 (1972) 95.

[132] J. Ballam et al., "Vector meson production by polarized photons at $2.8 \mathrm{GeV}, 4.7 \mathrm{GeV}$, and 9.3 GeV", Phys. Rev. D7 (1973) 3150.

[133] CLAS, I. Hleiqawi et al., "Cross Sections for the $\gamma p \rightarrow K^{* 0} \Sigma^{+}$Reaction at $E_{\gamma}=1.7-3.0$ GeV”, Phys. Rev. C75 (2007) 042201. 
Inequalities on spin observables

[134] CLAS, K. McCormick et al., "Tensor polarization of the $\Phi$ meson photoproduced at high $t$ ", Phys. Rev. C69 (2004) 032203.

[135] H.J. Halpern et al., "Measurement of the asymmetry parameter in the photoproduction of $\phi$ mesons", Phys. Rev. Lett. 29 (1972) 1425.

[136] J. Ajaka et al., "Evidence for nucleon-resonance excitation in omega-meson photoproduction”, Phys. Rev. Lett. 96 (2006) 132003.

[137] A.I. Titov et al., "Photoproduction of $\Phi$ meson from proton: Polarization observables and the strangeness in the nucleon", Phys. Rev. C58 (1998) 2429.

[138] M. Pichowsky, C. Savkli and F. Tabakin, "Polarization observables in vector meson photoproduction”, Phys. Rev. C53 (1996) 593.

[139] J. Soffer and D. Wray, "Comments on spin effects in proton proton cross-section measurements", Phys. Lett. B43 (1973) 514.

[140] A. Yokosawa, "Reviews of experimental results from the polarized beam program at the Argonne ZGS", Phys. Rept. 64 (1980) 47.

[141] J.P. Stanley et al., "Measurements of $\Delta \sigma_{L}$ and $\Delta \sigma_{T}$ in pp scattering between $200 \mathrm{MeV}$ and $520 \mathrm{MeV}$ ", Nucl. Phys. A403 (1983) 525.

[142] I.P. Auer et al., "Measurement of $\Delta \sigma_{L}$ and $C(L, L)=(L, L: 0,0)$ in proton-proton scattering between $300 \mathrm{MeV}$ and $800 \mathrm{MeV}$ ", Phys. Rev. D29 (1984) 2435.

[143] M.G. Doncel and A. Mendez, "Spin rotation parameters for inclusive reactions", Phys. Lett. B41 (1972) 83.

[144] C. Bourrely and J. Soffer, "Positivity constraints on spin observables in hadronic inclusive reactions", Phys. Rev. D75 (2007) 117501.

[145] E704, A. Bravar et al., "Analyzing power measurement in inclusive $\Lambda^{0}$ production with a 200 GeV/c polarized proton beam”, Phys. Rev. Lett. 75 (1995) 3073.

[146] E704, A. Bravar et al., "Spin transfer in inclusive $\Lambda^{0}$ production by transversely polarized protons at 200-GeV/c", Phys. Rev. Lett. 78 (1997) 4003.

[147] L.G. Pondrom, "Hyperon experiments at Fermilab”, Phys. Rept. 122 (1985) 57.

[148] D. de Florian, M. Stratmann and W. Vogelsang, "Polarized Lambda baryon production in p p collisions", Phys. Rev. Lett. 81 (1998) 530.

[149] D. de Florian et al., "Bounds on transverse spin asymmetries for $\Lambda$ baryon production in pp collisions at BNL RHIC", Phys. Lett. B439 (1998) 176.

[150] J. Soffer, M. Stratmann and W. Vogelsang, "Accessing transversity in double-spin asymmetries at the BNL-RHIC", Phys. Rev. D65 (2002) 114024.

[151] G.R. Goldstein and J.F. Owens, "Polarization in inclusive reactions", Nucl. Phys. B103 (1976) 145.

[152] J. Soffer, "Positivity constraints on initial spin observables in inclusive reactions", Phys. Rev. Lett. 91 (2003) 092005.

[153] A. Mukherjee, M. Stratmann and W. Vogelsang, "Next-to-leading order QCD corrections to A(TT) for prompt photon production”, Phys. Rev. D67 (2003) 114006. 
[154] J. Soffer, "Positivity constraints for spin dependent parton distributions", Phys. Rev. Lett. 74 (1995) 1292.

[155] O. Martin et al., "Transverse double-spin asymmetries for muon pair production in $\mathrm{p} \mathrm{p}$ collisions", Phys. Rev. D60 (1999) 117502.

[156] J. Soffer and P. Taxil, "Polarization effects in massive lepton pair production", Nucl. Phys. B172 (1980) 106.

[157] D.W. Sivers, "Hard scattering scaling laws for single spin production asymmetries", Phys. Rev. D43 (1991) 261.

[158] J.C. Collins, "Fragmentation of transversely polarized quarks probed in transverse momentum distributions", Nucl. Phys. B396 (1993) 161.

[159] S.J. Brodsky, D.S. Hwang and I. Schmidt, "Final-state interactions and single-spin asymmetries in semi-inclusive deep inelastic scattering", Nucl. Phys. B642 (2002) 344.

[160] M. Anselmino, M. Boglione and F. Murgia, "Single spin asymmetry for $\mathrm{p}$ (polarized) $\mathrm{p} \rightarrow$ $\pi X$ in perturbative QCD”, Phys. Lett. B362 (1995) 164.

[161] M. Anselmino et al., "The general partonic structure for hadronic spin asymmetries", Phys. Rev. D73 (2006) 014020.

[162] I. Schmidt, J. Soffer and J.J. Yang, “Transverse single spin asymmetries in photon production", Phys. Lett. B612 (2005) 258.

[163] X. Artru, "Proposals for measuring transversity distributions in deep inelastic electron scattering and a model for E704 asymmetries", (1993), hep-ph/9310323, Talk at Int. Workshop on High Energy Spin Physics, Protvino, Russia, Sep 20-24, 1993.

[164] X. Artru, J. Czyzewski and H. Yabuki, "Single spin asymmetry in inclusive pion production, Collins effect and the string model", Z. Phys. C73 (1997) 527.

[165] N. Christ and T.D. Lee, "Possible Tests of $\mathrm{C}_{s t}$ and $\mathrm{T}_{s t}$ Invariances in $l^{ \pm}+\mathrm{N} \rightarrow l^{ \pm}+\Gamma$ and

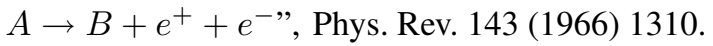

[166] M.G. Doncel and E. de Rafaël, "Inelastic lepton scattering from nucleons and positivity restrictions", Nuovo Cim. A4 (1971) 363.

[167] J. Soffer and O.V. Teryaev, "Positivity constraints and flavor dependence of higher twists", Phys. Lett. B490 (2000) 106.

[168] A. de Rujúla and E. de Rafaël, "Positivity restrictions on nucleon compton amplitudes", Annals Phys. 78 (1973) 132.

[169] X.D. Ji, "The Nucleon structure functions from deep inelastic scattering with electroweak currents", Nucl. Phys. B402 (1993) 217.

[170] A. de Rújula, M.G. Doncel and E. de Rafaël, "Coincidence electroproduction reactions and positivity restrictions", Phys. Rev. D8 (1973) 839.

[171] V. Dmitrasinovic, "Positivity restrictions in polarized coincidence electronuclear scattering", Phys. Rev. C51 (1995) 1528.

[172] C.P. Korthals-Altes, M. Perrottet and E. de Rafaël, "Inclusive muon production reactions as a test of neutral currents", Nucl. Phys. B76 (1974) 574. 
Inequalities on spin observables

[173] C.P. Korthals-Altes, M. Perrottet and E. de Rafaël, "Coincidence electroproduction reactions as a test of neutral currents", Nucl. Phys. B87 (1975) 527.

[174] C. Bourrely, J. Soffer and O.V. Teryaev, "Positivity constraints for lepton polarization in neutrino deep inelastic scattering”, Phys. Rev. D69 (2004) 114019.

[175] J. Callan, Curtis G. and D.J. Gross, "High-energy electroproduction and the constitution of the electric current", Phys. Rev. Lett. 22 (1969) 156.

[176] C.H. Albright, C. Jarlskog and L. Wolfenstein, "Neutrino production of $\mathrm{m}+$ and e+ heavy leptons. 2", Nucl. Phys. B84 (1975) 493.

[177] K. Sasaki, J. Soffer and T. Uematsu, "Positivity constraints on photon structure functions", Phys. Lett. B522 (2001) 22.

[178] K. Sasaki, J. Soffer and T. Uematsu, "Virtual photon structure functions and positivity constraints", Phys. Rev. D66 (2002) 034014.

[179] V.M. Budnev, V.L. Chernyak and I.F. Ginzburg, "Kinematics of gamma gamma scattering", Nucl. Phys. B34 (1971) 470.

[180] K. Sasaki, "QCD predictions for deep inelastic photon-photon scatterings", Phys. Rev. D22 (1980) 2143.

[181] S.D. Bass, S.J. Brodsky and I. Schmidt, "The spin structure of a polarized photon", Phys. Lett. B437 (1998) 417.

[182] R.L. Jaffe and X.D. Ji, "Chiral odd parton distributions and polarized Drell-Yan", Phys. Rev. Lett. 67 (1991) 552.

[183] M. Diehl and T. Gousset, "Time ordering in off-diagonal parton distributions", Phys. Lett. B428 (1998) 359.

[184] J. Soffer and O.V. Teryaev, "Gluon polarization in transversely polarized nucleons and jet spin asymmetries at RHIC", Phys. Rev. D56 (1997) 1353.

[185] A.S. Gorsky and B.L. Ioffe, "Anomalies, operator product expansion and structure functions in QCD”, Part. World. 1 (1990) 114.

[186] R. Ali and P. Hoodbhoy, "Gluonic transverse spin structure functions and their possible measurement in chi(2) production”, Z. Phys. C57 (1993) 325.

[187] J. Soffer and O.V. Teryaev, "A positivity bound for the longitudinal gluon distribution in a nucleon", Phys. Lett. B419 (1998) 400.

[188] O.V. Teryaev, "Positivity constraints in QCD", (1998), hep-ph/9808335, Contribution to $11^{\text {th }}$ Int. Conf. on Problems of Quantum Field Theory, ed. B.M. Barbashov et al., Dubna (1998).

[189] C. Bourrely and J. Soffer, "Do we understand the single-spin asymmetry for $\pi^{0}$ inclusive production in pp collisions?”, Eur. Phys. J. C36 (2004) 371.

[190] U. D' Alesio and F. Murgia, "Parton intrinsic motion in inclusive particle production: Unpolarized cross sections, single spin asymmetries and the Sivers effect", Phys. Rev. D70 (2004) 074009 .

[191] O.V. Teryaev, "Factorization and universality in spin-dependent SIDIS", Phys. Part. Nucl. 35 (2004) S24.

[192] R.L. Jaffe and A. Manohar, "Nuclear gluonometry", Phys. Lett. B223 (1989) 218. 
[193] A. Bacchetta et al., "Bounds on transverse momentum dependent distribution and fragmentation functions", Phys. Rev. Lett. 85 (2000) 712.

[194] A. Kotzinian, "New quark distributions and semiinclusive electroproduction on the polarized nucleons", Nucl. Phys. B441 (1995) 234.

[195] R.D. Tangerman and P.J. Mulders, "Probing transverse quark polarization in deep inelastic leptoproduction”, Phys. Lett. B352 (1995) 129.

[196] H. Avakian et al., "Pretzelosity distribution function $h_{1 T}^{\perp}$ and the single spin asymmetry $A_{U T}^{\sin \left(3 \phi-\phi_{S}\right),,}(2008), 0805.3355$.

[197] H. Meyer and P.J. Mulders, "Polarized and unpolarized structure functions in a diquark model for the nucleon", Nucl. Phys. A528 (1991) 589.

[198] W. Melnitchouk, A.W. Schreiber and A.W. Thomas, "Deep inelastic scattering from off-shell nucleons", Phys. Rev. D49 (1994) 1183.

[199] K. Suzuki and T. Shigetani, "Chiral-odd transversity spin structure function h1(x) of the nucleon in a constituent quark model", Nucl. Phys. A626 (1997) 886.

[200] R. Jakob, P.J. Mulders and J. Rodrigues, "Modelling quark distribution and fragmentation functions", Nucl. Phys. A626 (1997) 937.

[201] S.J. Brodsky et al., "Light-cone representation of the spin and orbital angular momentum of relativistic composite systems", Nucl. Phys. B593 (2001) 311.

[202] X.D. Ji and F. Yuan, "Parton distributions in light-cone gauge: Where are the final-state interactions?", Phys. Lett. B543 (2002) 66.

[203] J.C. Collins, "Leading-twist single-transverse-spin asymmetries: Drell-Yan and deepinelastic scattering", Phys. Lett. B536 (2002) 43.

[204] X. Artru and K. Benhizia, "The relativistic hydrogen atom: A theoretical laboratory for structure functions", (2005), hep-ph/0512161, in Transversity 2005, ed. V. Barone and Ph. Ratcliffe, Word Scientific (Singapore, 2006), Proc. Workshop on Transverse Polarisation Phenomena in Hard Processes, Como (Italy), 7-10 Sept. 2005.

[205] X. Artru and K. Benhizia, "The high- $Z$ hydrogen-like atom: a model for polarized structure functions", Problems of Atomic Science and Technology 3 (2007) 98, Talk given at Int. Conf. Quant. Electrodynamics and Stat. Phys., Kharkov (Ukraine), 19-23 Sept. 2006.

[206] HERMES, D. Hasch, “Spin physics at HERMES”, AIP Conf. Proc. 915 (2007) 307.

[207] COMPASS, V.Y. Alexakhin et al., "First measurement of the transverse spin asymmetries of the deuteron in semi-inclusive deep inelastic scattering", Phys. Rev. Lett. 94 (2005) 202002.

[208] COMPASS, E.S. Ageev et al., "A new measurement of the Collins and Sivers asymmetries on a transversely polarised deuteron target", Nucl. Phys. B765 (2007) 31.

[209] F. Bradamante, "The COMPASS experiment at CERN: Present and future", Nucl. Phys. Proc. Suppl. 154 (2006) 86.

[210] COMPASS, M. Alekseev et al., "Collins and Sivers Transverse Spin Asymmetries for Pions and Kaons on Deuterons", (2008), 0802.2160.

[211] FNAL-E704, D.L. Adams et al., "Analyzing power in inclusive $\pi^{+}$and $\pi^{-}$production at high $x_{F}$ with a $200 \mathrm{GeV}$ polarized proton beam", Phys. Lett. B264 (1991) 462. 
Inequalities on spin observables

[212] Fermilab E704, A. Bravar et al., "Single-spin asymmetries in inclusive charged pion production by transversely polarized antiprotons", Phys. Rev. Lett. 77 (1996) 2626.

[213] M. Boglione and E. Leader, "Reassessment of the Collins mechanism for single-spin asymmetries and the behavior of $\Delta d(x)$ at large $x "$, Phys. Rev. D61 (2000) 114001.

[214] U. D'Alesio and F. Murgia, “Azimuthal and Single Spin Asymmetries in Hard Scattering Processes”, Prog. Part. Nucl. Phys. 612008 (2008) 394.

[215] X. Artru, in Proc. RIKEN-BNL Workshop Future Transversity Measurements, Brookhaven, N.Y., Sept. 2000, RIKEN Proc. \# 29, p. 325; and Proc. Topical Workshop Transverse Spin Physics, DESY-Zeuthen, 9-11 July 2001 (Internal Report DESY Zeuthen 01-01, ed. by J. Blümlein, W.-D. Nowak, G. Schnell, Website: http://www.desy.de/spin01, p.300).

[216] A.V. Efremov, L. Mankiewicz and N.A. Törnqvist, "Jet handedness as a measure of quark and gluon polarization", Phys. Lett. B284 (1992) 394.

[217] O. Nachtmann, "A new tool for the study of fundamental interactions: parity odd correlations in quark fragmentation", Nucl. Phys. B127 (1977) 314.

[218] A.V. Belitsky and A.V. Radyushkin, "Unraveling hadron structure with generalized parton distributions", Phys. Rept. 418 (2005) 1.

[219] K. Goeke, M.V. Polyakov and M. Vanderhaeghen, "Hard exclusive reactions and the structure of hadrons", Prog. Part. Nucl. Phys. 47 (2001) 401.

[220] B. Pire, J. Soffer and O. Teryaev, "Positivity constraints for off-forward parton distributions", Eur. Phys. J. C8 (1999) 103.

[221] M. Diehl, "Generalized parton distributions with helicity flip", Eur. Phys. J. C19 (2001) 485.

[222] P.V. Pobylitsa, "Inequalities for generalized parton distributions $H$ and $E$ ", Phys. Rev. D65 (2002) 077504.

[223] M. Kirch, P.V. Pobylitsa and K. Goeke, "Inequalities for nucleon generalized parton distributions with helicity flip", Phys. Rev. D72 (2005) 054019.

[224] P.V. Pobylitsa, "Disentangling positivity constraints for generalized parton distributions", Phys. Rev. D65 (2002) 114015.

[225] P.V. Pobylitsa, "Positivity bounds on generalized parton distributions in impact parameter representation", Phys. Rev. D66 (2002) 094002.

[226] A.V. Radyushkin, "Scaling Limit of Deeply Virtual Compton Scattering", Phys. Lett. B380 (1996) 417.

[227] A.V. Radyushkin, "Asymmetric gluon distributions and hard diffractive electroproduction", Phys. Lett. B385 (1996) 333.

[228] A.V. Radyushkin, "Nonforward parton distributions”, Phys. Rev. D56 (1997) 5524.

[229] A.D. Martin and M.G. Ryskin, "The effect of off-diagonal parton distributions in diffractive vector meson electroproduction", Phys. Rev. D57 (1998) 6692.

[230] B.C. Tiburzi, "Estimates for pion photon transition distributions", Phys. Rev. D72 (2005) 094001.

[231] J.P. Lansberg, B. Pire and L. Szymanowski, "Exclusive meson pair production in gamma* gamma scattering at small momentum transfer", Phys. Rev. D73 (2006) 074014. 
[232] S. Friot, B. Pire and L. Szymanowski, "Deeply virtual Compton scattering on a photon and generalized parton distributions in the photon", Phys. Lett. B645 (2007) 153.

[233] E.A. Kuraev, L.N. Lipatov and V.S. Fadin, "The Pomeranchuk Singularity in Nonabelian Gauge Theories", Sov. Phys. JETP 45 (1977) 199.

[234] I.I. Balitsky and L.N. Lipatov, "The Pomeranchuk Singularity in Quantum Chromodynamics", Sov. J. Nucl. Phys. 28 (1978) 822.

[235] L.N. Lipatov, "The Bare Pomeron in Quantum Chromodynamics", Sov. Phys. JETP 63 (1986) 904.

[236] C. Bourrely, J. Soffer and O.V. Teryaev, “The $Q^{2}$ evolution of Soffer inequality”, Phys. Lett. B420 (1998) 375.

[237] C. Bourrely, E. Leader and O.V. Teryaev, "Positivity of NLO spin-dependent parton distributions", (1997), hep-ph/9803238.

[238] O.V. Teryaev, “The irreversibility of QCD evolution equations", Phys. Part. Nucl. 36 (2005) S160.

[239] L.N. Lipatov, "Leading logarithmic approximation in QCD”, Phys. Atom. Nucl. 67 (2004) 83.

[240] V.N. Gribov and L.N. Lipatov, "Deep inelastic e p scattering in perturbation theory", Sov. J. Nucl. Phys. 15 (1972) 438.

[241] G. Altarelli and G. Parisi, "Asymptotic freedom in parton language”, Nucl. Phys. B126 (1977) 298.

[242] Y.L. Dokshitzer, "Calculation of the structure functions for deep inelastic scattering and $\mathrm{e}^{+} \mathrm{e}^{-}$annihilation by perturbation theory in quantum chromodynamics. (in Russian)", Sov. Phys. JETP 46 (1977) 641.

[243] L.N. Lipatov, “The parton model and perturbation theory”, Sov. J. Nucl. Phys. 20 (1975) 94.

[244] A.P. Bukhvostov, L.N. Lipatov and N.P. Popov, "Parton distribution functions in perturbation theory", Yad. Fiz. 20 (1974) 532.

[245] J.C. Collins and J.W. Qiu, "A new derivation of the Altarelli-Parisi equations", Phys. Rev. D39 (1989) 1398.

[246] L. Durand and W. Putikka, "Probabilistic derivation of parton splitting functions", Phys. Rev. D36 (1987) 2840.

[247] A. Cafarella and C. Coriano, "The kinetic interpretation of the DGLAP equation, its KramersMoyal expansion and positivity of helicity distributions", Int. J. Mod. Phys. A20 (2005) 4863.

[248] O. Nachtmann, "Positivity constraints for anomalous dimensions", Nucl. Phys. B63 (1973) 237.

[249] C.H. Llewellyn Smith and S. Wolfram, "Positivity constraints on quark and gluon distributions in QCD”, Nucl. Phys. B138 (1978) 333.

[250] G.R. Goldstein, R.L. Jaffe and X.D. Ji, "Soffer’s inequality”, Phys. Rev. D52 (1995) 5006.

[251] V. Barone, "On the QCD evolution of the transversity distribution”, Phys. Lett. B409 (1997) 499. 
Inequalities on spin observables

[252] W. Vogelsang, "Next-to-leading order evolution of transversity distributions and Soffer's inequality", Phys. Rev. D57 (1998) 1886.

[253] S. Kumano and M. Miyama, "Two-loop anomalous dimensions for the structure function $h_{1}$ ”, Phys. Rev. D56 (1997) 2504.

[254] J. Jalilian-Marian et al., “The BFKL equation from the Wilson renormalization group”, Nucl. Phys. B504 (1997) 415.

[255] O.V. Teryaev, "QCD evolution and density matrix positivity", Proceedings of the XXX PNPI International Winter School, February, 2005, Repino, Russia, edited by V. Kudryavtsev and L. Lipatov, pp. 90-102, Gatchina, 2007, PNPI,

[256] A.V. Radyushkin, "Double distributions and evolution equations", Phys. Rev. D59 (1999) 014030 .

[257] J. Soffer and O.V. Teryaev, "Neutron spin-dependent structure function, Bjorken sum rule, and first evidence for singlet contribution at low x", Phys. Rev. D56 (1997) 1549.

[258] B.I. Ermolaev, M. Greco and S.I. Troyan, "Non-singlet structure functions: Combining the leading logarithms resummation at small $x$ with DGLAP”, Phys. Lett. B622 (2005) 93.

[259] D. Haidt, “ $F_{2}$ challenging DGLAP”, Eur. Phys. J. C35 (2004) 519.

[260] M. Gluck, C. Pisano and E. Reya, "The curvature of $F_{2}^{p}\left(x, Q^{2}\right)$ as a probe of the range of validity of perturbative QCD evolutions in the small $x$ region", Eur. Phys. J. C50 (2007) 29.

[261] G.M. Zaslavsky, "Stochasticity in quantum systems”, Phys. Rept. 80 (1981) 157.

[262] M. Ciafaloni, "Coherence Effects in Initial Jets at Small $q^{2} / s "$, Nucl. Phys. B296 (1988) 49.

[263] S. Catani, F. Fiorani and G. Marchesini, "QCD Coherence in Initial State Radiation”, Phys. Lett. B234 (1990) 339.

[264] S. Catani, F. Fiorani and G. Marchesini, "Small $x$ Behavior of Initial State Radiation in Perturbative QCD”, Nucl. Phys. B336 (1990) 18.

[265] G. Marchesini, "QCD coherence in the structure function and associated distributions at small $x "$, Nucl. Phys. B445 (1995) 49.

[266] J.C. Collins, "Spin correlations in Monte-Carlo event generators", Nucl. Phys. B304 (1988) 794.

[267] I.G. Knowles, “Angular correlations in QCD”, Nucl. Phys. B304 (1988) 767.

[268] P. Richardson, "Spin correlations in Monte Carlo simulations", JHEP 11 (2001) 029.

[269] A. Wehrl, "General properties of entropy”, Rev. Mod. Phys. 50 (1978) 221.

[270] B.M. Terhal, "Detecting quantum entanglement”, Theor. Comput. Sci. 287 (2002) 313.

[271] C.D. Jeffries, "History of the development of polarized targets", in High energy spin physics, Proc. 9th Int. Symp., Bonn (1990), vol. 2, ed. W.T. Meyer, (Springer, Berlin, 1991), p. 3-19.

[272] E. Steffens and W. Haeberli, "Polarized gas targets", Reports on Progress in Physics 66 (2003) 1887.

[273] G. Moortgat-Pick, "Physics aspects of polarized $\mathrm{e}^{+}$at the linear collider", (0700), hepph/0607173, Talk given at the POSIPOL2006 Workshop, CERN, April 2006. 
[274] K. Sakaue et al., "Polarized positron generation based on laser Compton scheme and its polarization measurements", Int. J. Mod. Phys. B21 (2007) 519.

[275] A.D. Krisch, "Summary of workshop on polarized anti-proton sources, April 18-21, 1985, Bodega bay, California, USA", Presented at Workshop on Polarized Anti-Proton Sources, Bodega Bay, CA, Apr 18-21, 1985, AIP Series \# 145.

[276] FNAL-E581/704, D.P. Grosnick et al., "The design and performance of the FNAL highenergy polarized beam facility", Nucl. Instrum. Meth. A290 (1990) 269.

[277] SING, A. Martin et al., "Polarization at small angles in anti-proton - carbon elastic scattering at lear energies", Nucl. Phys. A487 (1988) 563.

[278] C.B. Dover et al., "Antinucleon nucleus inelastic scattering and the spin dependence of the $\overline{\mathrm{N} N}$ annihilation potential", Phys. Lett. B143 (1984) 45.

[279] C.B. Dover, "Antiproton nucleus inelastic scattering and the spin-isospin dependence of the $\bar{N} N$ interaction", in Physics with antiprotons at LEAR in the ACOL era, Proceedings 3rd LEAR Workshop, Tignes, France, Jan 19-26, 1985, Edited by U. Gastaldi, R. Klapisch, J.-M. Richard, J. Tran Thanh Van (Éditions Frontières, Gif-sur-Yvette,1985).

[280] C.B. Dover, "Antinucleon - nucleus interactions", in [291].

[281] Z. Yu-shun et al., "Spin effect of anti-proton - nucleus inelastic scattering", Phys. Rev. C60 (1999) 054618.

[282] Y. Onel, A. Penzo and R. Rossmanith, "A spin splitter for antiprotons in LEAR", in Fundamental symmetries, edited by P. Bloch, P. Pavlopoulos and R. Klapisch, pp. 339-345, Erice, 1987, (1st Ettore Majorana International School of Physics with Low-energy Antiprotons), Plenum, New-York.

[283] SPLIT, A. Penzo et al., "Perspectives for polarized anti-protons at LEAR: The Spin Splitter", in [291], p. 183-194.

[284] M. Conte et al., "Further considerations regarding the Spin Splitter", in [291], p. 211-216.

[285] R. Rossmanith, "Status of the spin splitter experiment at IUCF", in 'High energy spin physics, Proc. 9th Int. Symp., Bonn (1990), vol. 2, ed. W.T. Meyer, (Springer, Berlin, 1991), p. 121123.

[286] F. Rathmann et al., "A method to polarize stored antiprotons to a high degree", Phys. Rev. Lett. 94 (2005) 014801.

[287] A.I. Milstein and V.M. Strakhovenko, "Polarizing mechanisms for stored $p$ and $\bar{p}$ beams interacting with a polarized target", Phys. Rev. E72 (2005) 066503.

[288] J.-M. Richard, "Spin dependence in $\overline{\mathrm{N} N}$ scattering at low-energy", CERN-TH-2978, Oct 1980, in International Symposium on High Energy Physics with Polarized Beams and Polarized Targets, Lausanne, Switzerland, Sep 25 - Oct 1, 1980. C. Joseph and J. Soffer (eds.); Birkhauser, Experientia Supplementum, v. 38 (1981) p. 535-540.

[289] J.-M. Richard, "Spin dependence in $\overline{\mathrm{N}} \mathrm{N}$ at low-energy", Lecture given at Int. Sch. of Physics of Exotic Atoms: Workshop on Physics at LEAR with Low-Energy Cooled Antiprotons, Erice, Sicily, May 9-16, 1982, ed. U. Gastaldi and R. Klapisch, Plenum Press, N.Y. (1984) p. 443445 .

[290] C.B. Dover and J.-M. Richard, "Spin observables in low-energy nucleon anti-nucleon scattering", Phys. Rev. C25 (1982) 1952. 
Inequalities on spin observables

[291] Physics at LEAR with low energy antiprotons, Proc. 4th LEAR Workshop, Villars-sur-Ollon, Switzerland, 1988, Harwood, Chur.

\section{List of Tables}

1 Normalised cross section matrix $\widehat{\mathcal{R}}$ of $1 / 2+0 \rightarrow 1 / 2+0$ (Tr $\widehat{\mathcal{R}}=4)$. Axis labels are $\{1,2,3\}=\{m, n, l\}$ in the helicity basis, $\{l, m, n\}$ in the transversity basis. $(0-3 \mid 1+i 2)$, for instance, is a condensed notation for $(0 \mid 1)+i(0 \mid 2)-(3 \mid 1)-i(3 \mid 2)$, which may be seen as $\left\langle\left(\mathbb{1}-\sigma_{3}\right) \otimes\left(\sigma_{1}+i \sigma_{2}\right)\right\rangle$, according to Eq. (2.67). . . . . . 25

2 Spin observables for $\pi^{-}$p elastic scattering, as measured by Abaev et al. [81] at

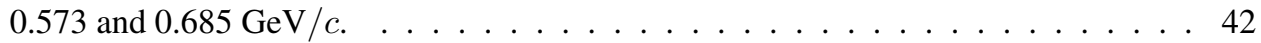

3 Spin observables for $\pi^{+}$p elastic scattering, as measured by Supek et al. [82] at $0.657 \mathrm{GeV} / c \ldots \ldots \ldots \ldots \ldots \ldots \ldots$

4 Domain allowed for pairs of observables: the entire square $(\square)$, the unit disk $(\bigcirc)$, the triangle $\left|2 \mathcal{O}_{1}\right| \leq \mathcal{O}_{2}+1(\nabla)$, or $\left|2 \mathcal{O}_{2}\right| \leq \mathcal{O}_{1}+1(\triangleleft)$, where $\mathcal{O}_{1}$ is horizontal and $\mathcal{O}_{2}$ vertical. The symbol $\otimes$ indicates that the pair of observables is constrained in the unit disk, but the corresponding operators do not anticommute. . . . . . . . 49

5 Submatrix $\mathcal{R}_{3}^{+}$of the Cross Section Matrix of $\overline{\mathrm{p}}+\mathrm{p} \uparrow \rightarrow \bar{\Lambda} \uparrow+\Lambda \uparrow \ldots \ldots \ldots$. . . . 57

6 Submatrix $\widehat{\mathcal{R}}^{\mp}$ of the renormalised cross-section matrix $\widehat{\mathcal{R}}$. The upper sign refers to pseudoscalar photoproduction, the lower one to scalar. . . . . . . . . . . . 67

\section{List of Figures}

1 Axes to project out the spin components of each particle in the centre-of-mass frame of the reaction $a+b \rightarrow c+d \ldots \ldots \ldots \ldots \ldots$

2 The scattering plane is rotated by $\phi$ with respect to the frame defined by the target polarisation and the beam axis. . . . . . . . . . . . . . . 10

3 Positivity cone $\mathcal{C}$ for matrices of arbitrary trace and positivity domain $\mathcal{D}$ for unittrace matrices. $P$ is the hyperplane $\operatorname{Tr}(\rho)=1$. The decomposition $\rho=\rho_{\|}+\rho_{\perp}$ of Eq. (2.15) is also schematically represented. . . . . . . . . . . . . . . . 14

4 Schematic drawing of a 2-dimensional polar transform. . . . . . . . . . . . . . 16

5 Mechanism for the reaction $\pi^{+}+{ }^{4} \mathrm{He} \rightarrow \pi^{0}+\mathrm{p}+{ }^{3} \mathrm{He}$. The double arrows represent intermediate and final polarisations in the forbidden case $\boldsymbol{S}_{p}=-\boldsymbol{S}_{n} . \ldots 27$

6 Spin observables for $\pi^{-}$p elastic scattering at 0.573 and $0.685 \mathrm{GeV} / c$ (left), and for $\pi^{+}$p elastic scattering at $0.657 \mathrm{GeV} / c$ (right), in axes $\left\{R, A, P_{n}\right\}$ compared to the unit sphere. The data are from Abaev et al. [81] for $\pi^{-} \mathrm{p}$ and Supek et al. [82] for $\pi^{+} \mathrm{p}$, and are shown with the error bars on the three observables. . . . . . . . . 42

7 Analysing power for $\bar{p} \mathrm{p} \rightarrow \pi^{-} \pi^{+}$at selected values of the antiproton momentum in the target frame. The data are from the PS172 collaboration [86]. . . . . . . . . . . . 44

8 Analysing power for $\overline{\mathrm{p}} \mathrm{p} \rightarrow \mathrm{K}^{-} \mathrm{K}^{+}$at selected values of the antiproton momentum in the target frame. The data are from the PS172 collaboration [86]. . . . . . . . . . 45

9 Results of PS 185 [94] on $\overline{\mathrm{p} p} \rightarrow \bar{\Lambda} \Lambda$ for the polarisation, and initial-state spin correlations, out of which the spin-singlet fraction $F_{S}$ can be estimated. This corresponds to one of the last runs, at $p_{\text {lab }}=1.637 \mathrm{GeV} / c$. Earlier measurements, obtained without polarised target, are in good agreement. . . . . . . . . . . . . 46

10 Some of the results of PS 185 [94] for the analysing power, depolarisation and transfer of polarisation of the reaction $\overline{\mathrm{p}} \mathrm{p} \rightarrow \bar{\Lambda} \Lambda$ at $p_{\text {lab }}=1.637 \mathrm{GeV} / c \ldots \ldots 47$

11 Random simulation of $P_{n}$ vs. $A_{n}$ (square), $A_{n}$ vs. $D_{m m}$ (disk) and $P_{n}$ vs. $C_{n n}$

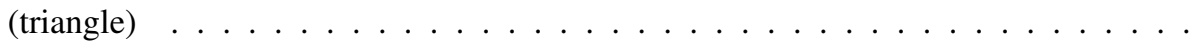


12 Same as Fig. 11, but each real or imaginary part amplitude is forced to be either 0 or $\pm 1 . \ldots \ldots \ldots \ldots \ldots \ldots \ldots \ldots \ldots \ldots \ldots \ldots \ldots \ldots \ldots \ldots \ldots \ldots$

13 Some allowed domains encountered in simulating randomly three observables: the unit sphere (a), the intersection of three orthogonal cylinders of unit radius (b), the intersection of two cylinders (c), or a slightly smaller double cone (d), a cylinder (e), a cone (f), a pyramid (g), a tetrahedron (h), an octahedron (i), a "coffee filter" (j), an inverted tent (k), and the intersection of two cylinders and a dihedral (l). For clarity, part of the limiting surface is sometimes removed. Some figures transformed by parity with respect to the centre of the cube or by interchange of the axes are also obtained. . . . . . . . . . . . . . . . . . .

14 Simulation of the domain allowed for the observables $\left\{P_{n}, D_{m m}, C_{l l}\right\}$ (left) and $\left\{C_{m l}, C_{m m}, A_{n}\right\}$ (right), using amplitudes whose real and imaginary parts are cho-

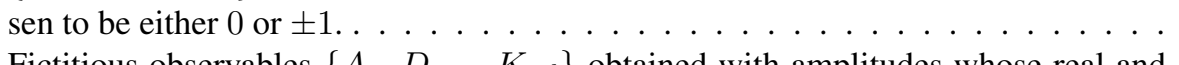

15 Fictitious observables $\left\{A_{n}, D_{m m}, K_{m l}\right\}$ obtained with amplitudes whose real and imaginary parts are chosen to be either 0 or \pm 1 , shown against the unit sphere (left) or the intersection of the three unit cylinders. . . . . . . . . . . . . 52

16 The domain for $\{x, y, z\}=\left\{P_{n}, D_{m m}, A_{n}\right\}$ (left) is inside the intersection of two cylinders, while the domain for $\left\{C_{m l}, C_{m m}, C_{l l}\right\}$ (right) is inside a slightly smaller double cone. For clarity, only the lower part of the boundary is shown. . . . . . . . 53

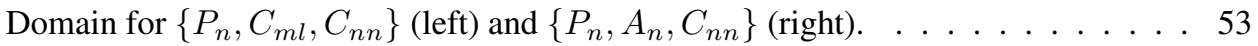
Domain for $\left\{P_{n}, A_{n}, D_{n n}\right\}$ (left) and $\left\{C_{n n}, C_{m m}, C_{l l}\right\}$ (right). . . . . . . 54 Observables $\left\{P_{n}, C_{m m}, C_{n n}\right\}$ (left) and $\left\{C_{m m}, C_{l l}, C_{n m m}\right\}$ (right). . . . . . 54 Simulation by random amplitudes of the observables $\left\{C_{m l}, C_{n l m}, C_{n n}\right\}$ (left) and

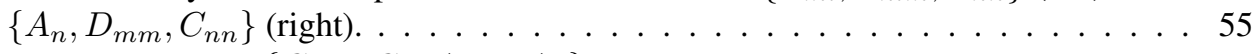

21 Any triple out of $\left\{C_{m m}, C_{l l}, A_{m m}, A_{l l}\right\}$ is constrained within an octahedron obtained by slicing off two opposite corners of the unit cube. . . . . . . . . . . 55 55

22 Polar transform (right) of the funnel-shape filter (left) encountered as the allowed domain of some triples of observables, e.g., $\left\{P_{n}, C_{m m}, C_{n n}\right\} . \ldots 60$ Analysing power $A_{n}$ and spin-spin correlation $A_{n n}$ in proton-proton scattering at $90^{\circ}$, as a function of the beam momentum. . . . . . . . . . . . 62 Beam asymmetry for $\gamma \mathrm{p} \rightarrow \eta \mathrm{p}$ at 2.5 and $3 \mathrm{GeV}$, as measured by Bussey et al. [112] 63 Beam asymmetry for $\gamma \mathrm{p} \rightarrow \pi^{0} \mathrm{p}$ at several photon energies, as measured by GRAAL [118]. 63

26 The magnitude of the $\Lambda$ hyperon polarisation observable vector $\sqrt{P^{2}+C_{x}^{2}+C_{z}^{2}}$ from recent CLAS data [119]. . . . . . . . . . . . . . . . . . . . . . 64

Tetrahedron domain limited by the inequalities (3.67) for the observables $x=A$,

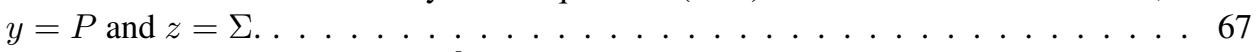
Tensor polarisation coefficient $\rho_{00}^{0}$ in $\gamma+\mathrm{p} \rightarrow \phi+\mathrm{p}$, as measured at CLAS [134]. . 71

Kinematics for the $\gamma+\mathrm{N} \rightarrow V+B^{\prime}$ and $V \rightarrow M_{1} M_{2}$ reactions. . . . . . . . 71

Domain allowed for $\Delta \sigma_{L}$ and $\Delta \sigma_{T} \ldots \ldots \ldots \ldots \ldots$

31 The allowed domain corresponding to the constraints Eq. (4.12) (left). The slice of the full domain for $D_{N N}=0$ ( middle) and for $D_{N N}=1 / 3($ right $)$. . . . . . . 79

32 For $\tau^{+}$production, $P_{P}$ versus $P_{L}$ in a domain limited by $R_{+} \geq 0, P \leq 1$ (grey dots inside disk) plus non trivial positivity constraints (black dots inside disk). From top to bottom and left to right, $E_{\nu}=10 \mathrm{GeV}, Q^{2}=1 \mathrm{GeV}^{2}, x=0.25,0.6,0.9, E_{\nu}=$ $10 \mathrm{GeV}, Q^{2}=4 \mathrm{GeV}^{2}, x=0.4,0.6,0.9, E_{\nu}=20 \mathrm{GeV}, Q^{2}=1 \mathrm{GeV}^{2}, x=$ $0.25,0.6,0.9, E_{\nu}=20 \mathrm{GeV}, Q^{2}=4 \mathrm{GeV}^{2}, x=0.25,0.6,0.9 . \ldots \ldots \ldots$
For $\tau^{+}$production, upper and lower bounds on $P_{P}$ (open circles) and $P_{L}$ (full cir-

33 For $\tau^{+}$production, upper and lower bounds on $P_{P}$ (open circles) and $P_{L}$ (full circles) as a function of $Q^{2}$ for $E_{\nu}=10 \mathrm{GeV}$ and $x=0.25,0.6,0.9 \ldots \ldots 88$

34 For $\tau^{+}$production, $P_{P}$ versus $P_{L}$ in a domain limited by $R_{+} \geq 0, P \leq 1$ assuming the Callan-Gross (left) or the Albright-Jarlskog (right) relations (grey dots inside disk) plus non trivial positivity constraints (black dots inside disk). $E_{\nu}=$ $10 \mathrm{GeV}, Q^{2}=1 \mathrm{GeV}^{2}$, from top to bottom, $x=0.25,0.6,0.9 \ldots \ldots \ldots$ 
35 Virtual photon-photon forward scattering with momenta $q(p)$ and helicities $a(b)$ and

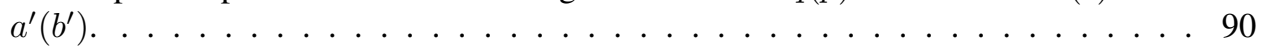

36 The allowed domain corresponding to the constraint Eq. (4.75) . . . . . . . . . . . . 94

37 Successive steps of the Monte-Carlo algorithm generating momentum correlations in cascade reactions. a) reactions $\mathrm{e}^{+} \mathrm{e}^{-} \rightarrow \tau^{+} \tau^{-}, \tau^{+} \rightarrow \mu^{+} \nu_{\mu} \bar{\nu}_{\tau}$ and $\tau^{-} \rightarrow \mu^{-} \bar{\nu}_{\mu} \nu_{\tau}$; b) a more complicated tree diagram. Downward arrows represent density matrices, upward arrows represent acceptance matrices. . . . . . . . . . . . . . . . 109

38 Relative positions of the classical positivity domain $\mathcal{D}^{\text {cl }}$ : the separability domain $\mathcal{S}$, the true positivity domain $\mathcal{D}$ and the domain of positive partial transform $\mathcal{D}^{\text {pt }}$ (dashed contour). $\mathcal{D} \cap \mathcal{D}^{\mathrm{pt}}$ is generally larger than $\mathcal{S}$, but for two spin one-half, one has $\mathcal{D} \cap \mathcal{D}^{\mathrm{pt}}=\mathcal{S}$. A matrix $\eta$ of the boundary $\partial \mathcal{S}$ is represented together with its reciprocal polar line, which is tangent to $\partial \mathcal{D}^{\mathrm{cl}}$. . . . . . . . . . . . 113

39 Classical positivity domain (cube), true positivity domain (tetrahedron) and separability domain (octahedron) for the triple $\left\{C_{x x}, C_{y y}, C_{z z}\right\}$ of observables. . . . . 114 